\title{
Onorden
}

\section{Youth unemployment and inactivity}

A comparison of school-to-work transitions and labour market outcomes in four Nordic countries

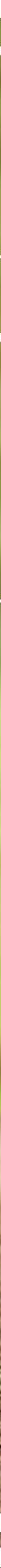



2 norden 



\section{Youth unemployment and inactivity}

A comparison of school-to-work transitions and labour market outcomes in four Nordic countries

Karsten Albæk, Rita Asplund, Erling Barth, Lena Lindahl, Kristine von Simson and Pekka Vanhala 
Youth unemployment and inactivity

A comparison of school-to-work transitions and labour market outcomes in four Nordic countries Karsten Albæk, Rita Asplund, Erling Barth, Lena Lindahl, Kristine von Simson and Pekka Vanhala

ISBN 978-92-893-4229-2 (PRINT)

ISBN 978-92-893-4230-8 (PDF)

ISBN 978-92-893-4231-5 (EPUB)

http://dx.doi.org/10.6027/TN2015-548

TemaNord 2015:548

ISSN 0908-6692

(C) Nordic Council of Ministers 2015

Layout: Hanne Lebech

Cover photo: ImageSelect

Print: Rosendahls-Schultz Grafisk

Printed in Denmark

This publication has been published with financial support by the Nordic Council of Ministers. However, the contents of this publication do not necessarily reflect the views, policies or recommendations of the Nordic Council of Ministers.

www.norden.org/nordpub

\section{Nordic co-operation}

Nordic co-operation is one of the world's most extensive forms of regional collaboration, involving Denmark, Finland, Iceland, Norway, Sweden, and the Faroe Islands, Greenland, and Åland.

Nordic co-operation has firm traditions in politics, the economy, and culture. It plays an important role in European and international collaboration, and aims at creating a strong Nordic community in a strong Europe.

Nordic co-operation seeks to safeguard Nordic and regional interests and principles in the global community. Common Nordic values help the region solidify its position as one of the world's most innovative and competitive.

\section{Nordic Council of Ministers}

Ved Stranden 18

DK-1061 Copenhagen K

Phone (+45) 33960200

www.norden.org 


\section{Contents}

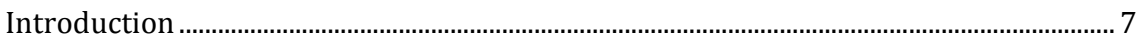

Increasing focus on school-to-work transitions........................................................... 7

Key features of the study...........................................................................................10

Structure and content of the present report................................................................12

1. Performance of young people - an international perspective ....................................17

1.1 Setting the stage ....................................................................................................17

1.2 Snapshot of recent Nordic comparative studies..............................................20

1.3 The Nordic story told by international comparative indicators....................21

2. General description of post-compulsory-school activities .........................................37

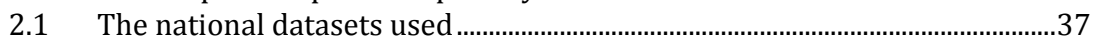

2.2 Main activities of young people - the overall pattern ...................................... 41

2.3 Non-completers - an introductory view............................................................65

2.4 Individual school-to-work profiles - a general picture .....................................76

3. School-to-work trajectories: country-specific cluster results .....................................89

3.1 The cluster analysis method - a brief outline...................................................... 89

3.2 Country-specific cluster results: all young people ...........................................98

3.3 Country-specific cluster results: non-completers..........................................113

4. School-to-work trajectories: common Nordic cluster results .................................129

4.1 Common Nordic trajectories and clusters: all young people ........................130

4.2 Common Nordic trajectories and clusters: non-completers ........................136

5. Non-completers' school-to-work trajectories: stylized cluster results ...................147

6. Labour market outcomes as young adults...................................................................171

6.1 Main activities beyond age 21 - all young people ..........................................171

6.2 Main activities beyond age 21 - young men vs. young women....................181

6.3 Main activities beyond age 21 - completers vs. non-completers................190

6.4 Main activities beyond age 21 - late completion vs. non-completion.......210

7. Labour market outcomes in view of background........................................................223

7.1 Gender and labour market outcomes ..............................................................223

7.2 Cohort and labour market outcomes .............................................................222

7.3 Family background and labour market outcomes.........................................232

7.4 Early pathways through education and labour market outcomes ............241

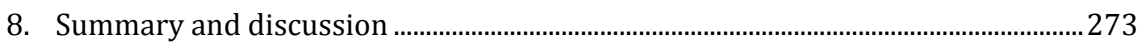

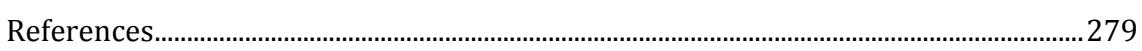

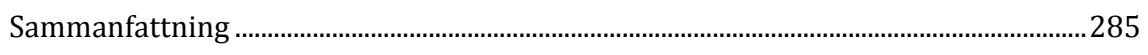

Appendix: Descriptions of national datasets used ..........................................................293

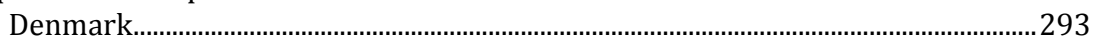

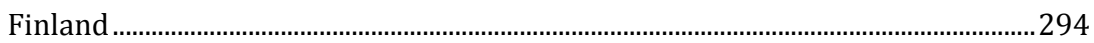

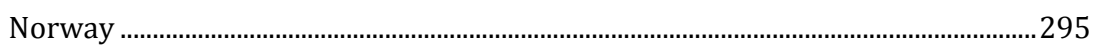

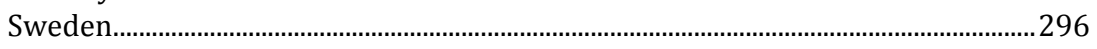





\section{Introduction}

Youth unemployment has for several decades been a much debated topic in both the academic and the political arena. The recent global economic crisis, in combination with profound changes in labour market structures (see e.g. Acemoglu and Autor, 2011), shifted youth unemployment to the top of the political agenda after alarming reports on surging youth unemployment rates and growing risks of young people becoming economically and socially marginalised (e.g. Scarpetta et al., 2010; ILO, 2011, 2013; Räisänen, 2013). Concomitantly, increasing attention was paid to so-called NEETs, that is, young people not in employment, education or training. Several recent reports highlight the prevalence of this phenomenon and also provide estimates of the societal costs of early school leaving and NEETs (e.g. Eurofound, 2012; Brunello and De Paola, 2014). Other studies highlight the complexity of problems often associated with being a NEET. A survey of the Prince Trust (2010) covering young people aged 16-25 reports that NEETs are significantly more likely to feel ashamed, rejected, lost, anxious, insecure, down and depressed, isolated and unloved. They are also disproportionately more likely to say that they had turned to drugs and that their life had no direction.

\section{Increasing focus on school-to-work transitions}

A growing body of evidence identifies early school leaving and school dropout as major reasons underlying youth unemployment and marginalisation from working life in a world where an upper secondary certificate has increasingly become the minimum requirement for proper access to the job market. Those left behind at the lowest floor - the low- or unskilled school leavers lacking work experience - are the losers in the competition with the better skilled. Their disproportionate presence among those holding temporary jobs, in combination with their high concentration in cyclically sensitive industries, makes them especially vulnerable. Apart from a much higher risk of becoming unemployed, as compared to their higher-educated peers, young people with a low education also face a much higher risk of repeated spells of unemployment 
that, moreover, tend to prolong over time. Ultimately school leavers end up outside the labour force for shorter or longer periods of time.

Hence, low educational attainment levels not only impede initial insertion into the labour market, but also represent an enduring barrier to employment (e.g. OECD, 2008a). Indeed, while the negative labour market and social outcomes of youth unemployment of a more temporary nature have been shown to diminish over time, there is a considerable body of evidence on long-term unemployment having lasting scarring effects on young people in terms of both future employment and future wages. Illustrative examples are a study by Kahn (2010) for the USA showing that graduating from college in a bad economy has large, negative and persistent effects on wages, and a study by Bell and Blanchflower (2009) for the UK reporting that youth unemployment raises unemployment, lowers wages, worsens health and lowers job satisfaction still 25 years later. Studies reporting permanent employment and wage losses are also found for other European countries, including Germany (e.g. Schmillen and Umkehrer, 2013; Möller and Umkehrer, 2014), Norway (e.g. Nilsen and Holm Reiso, 2014) and Sweden (e.g. Nordström Skans, 2011). Taken together, the existing evidence implies that the long-term costs of early exit from the education system have increased.

While the economic business cycle tends to have long-lasting career consequences for young labour market entrants, also the schooling decision itself may be affected by the state of the labour market. Desire to work may be an important motivation for some youth to end their education at an earlier stage. According to standard human capital theory (Becker, 1964), the economic situation affects the schooling decision mainly through the opportunity cost of schooling, measured as foregone earnings. A booming labour market implies more job openings and higher wages, which in turn increase the opportunity cost of schooling. This may encourage some youth to take advantage of the favourable labour market conditions and leave school on a temporary or permanent basis. An economic downturn, on the other hand, may induce youth to stay in school and postpone their labour market entry, or to return to education. Previous research on upper secondary schooling supports this prediction: Students are less likely to stay in school in good times. Also enrolment tends to decrease in good times (Clark, 2011), with graduation rates being countercyclical, as well (Reiling and Strøm, 2015). Additionally, the propensity to interrupt the education and drop out from school altogether increases in economic upturns (von Simson, 2014). On the whole, though, the association is not very strong and other factors, such 
as parental education, are more important in explaining schooling decisions than is the economic situation.

Nonetheless, early school leaving and dropout from upper secondary education can be seen to typically represent unsuccessful transitions within the school system and further into working life. This contention has, more recently, broadened the focus on unemployment and marginalisation among young people to the role of school-to-work transitions. Indeed, the transition from education to working life has frequently been argued to be the most critical phase in terms of a young person's labour market outcomes later in life. While these transitions evidently reflect the family situation and compulsory-school outcomes, these much researched background factors cannot offer a full explanation for young people's highly different school-to-work-transition patterns and, ultimately, for their differences in labour market outcomes in adulthood. The transitions in themselves seem to be of importance, as well. In other words, it seems reasonable to assume that young people's early school and labour market experiences upon leaving compulsory school also play a role for their labour market outcomes later in life.

These contentions explain and motivate the focus of our study on four Nordic countries - Denmark, Finland, Norway and Sweden - the main results of which are reported in this volume. More precisely, first we present and discuss the school-to-work transitions of young people leaving compulsory school. Next we use this information to indicate the extent to which different school-to-work-transition patterns are related to the probability of young people ending up in alternative labour market situations in adulthood. Doing so, we also include basic information on family background, which allows us to test whether or not early postcompulsory-school experiences have a "signal value of their own" especially when it comes to young people's probability of going into risky labour-market tracks dominated by time spent in NEET activities, that is, in unemployment or entirely outside the labour force. Put differently, is it evident that, in addition to family background, also early school-towork transitions contain important information concerning the likelihood of different labour market outcomes later in life? The rest of this chapter provides a more detailed outline of our study and the structure of the present report. 


\section{Key features of the study}

The labour market performance of youth is commonly described by use of various rather conventional indicators highlighting phenomena such as early school leaving, premature training dropout, youth employment and unemployment, and NEET. These indicators share the feature of providing an instantaneous picture of the educational outcome or of the position/status of youth in the labour market. The mediated picture can, as a consequence, be partly misleading as these static indicators fail to see the observed outcome "as a cumulative process of disengagement or withdrawal that occurs over time" (Lyche, 2010, p. 14). Emerging indicators, providing a more dynamic description of youth labour market performance, can correct for some of the shortcomings characterising static indicators but share, nonetheless, the common drawback of oversimplifying the dynamic nature of youth school-to-work trajectories.

In order to overcome this unsatisfactory situation, growing numbers of researchers have used alternative ways to assess comprehensively the multiple patterns of school-to-work transitions that youth are known to face when moving from education into working life. However, these transitions from education to work are far from easy to measure because of the "fluidity of the youth labour market" (OECD 2008b, p. 59). In particular, school-to-work transitions are often long-lasting processes involving frequent status changes between education, temporary jobs, unemployment and inactivity (Müller and Gangl, 2003; Wolbers, 2007; Saar et al., 2008). This also explains why transitions from education to work cannot be fully understood by analysing single changes of status only.

More recent research on school-to-work transitions is therefore increasingly based on longitudinal data allowing young people to be followed after they have completed their education over a longer time period. However, apart from high-quality longitudinal data, such a dynamic approach also requires the use of proper statistical methods. Since young people often shift between education, inactivity, unemployment and work before getting a stable job, sequence and cluster analysis has often been argued to be the most appropriate methodological approach in this context (e.g. Brzinsky-Fay, 2007). A strong advantage of this sociological method is that it allows entire individual school-to-work trajectories, including nature of spells and their order, to be identified, compared and classified into one of several distinct types of youth trajectories. This categorisation also allows proper account to be made for the fact that youth trajectories often show a high degree of diversity especially by gender and educational background. Accordingly, this evidence 
clearly improves our understanding of young people's often bumpy, occasionally unsuccessful, transition into employment.

As already noted, this dynamic approach to analysing school-to-work trajectories requires access to high-quality longitudinal data. It is, therefore, hardly surprising that this kind of evidence is, so far, available only for a small number of countries, occasionally for the whole of Europe as averages using information on a limited number of European countries, as done in Quintini and Manfredi (2009). Also missing is broad-based comparative information for individual Nordic countries, a knowledge gap that the present report aims to fill. Indeed, this report is, to our knowledge, the first contribution using sequence and cluster analysis to investigate school-to-work transitions for full cohorts of young people and, moreover, to also undertake cross-country comparisons of such transitions among young people based on comparative national data.

In exploring the educational and labour market experiences of young people, we pay particular attention to identifying risky trajectories and conspicuous differences in these respects between youth differing in their educational background, while also differentiating across genders. Additionally, attempts are made to identify and quantify possible changes in early transition patterns within countries by comparing key outcomes for a total of three youth cohorts: 16-year-olds in 1993, 1998 and 2003 , respectively. All three cohorts are traced up to the year $2008 \mathrm{im}-$ plying that the shortest follow-up period covers 5 years and the longest 15 years. Equally important, school-to-work-transition patterns and changes in these patterns are compared across the four Nordic countries under study to highlight major similarities and dissimilarities in "typical" school-to-work trajectories.

The analysis briefly outlined above provides detailed cross-Nordic information on distinct types of school-to-work trajectories for our three cohorts of 16-year-olds, up to age 20. Finally, this information is linked to the young persons' labour market outcomes at three points later in time: when they turn 21, 26 and 31. Throughout, these alternative labour market activities are grouped into five broad categories - full-time studying, employment, unemployment, disability benefits and "other" (inactivity). Apart from simply describing these relations between early and later labour market outcomes, we also undertake statistical analysis by use of so-called multinomial logit techniques, with a view of highlighting the relative strength between different early school-to-worktransition patterns and alternative labour market outcomes later as a young adult. As explained earlier, we thereby also account for family background in order to explore whether the link observed between ear- 
ly school-to-work-transition patterns and alternative labour market outcomes in adulthood remains approximately unchanged, diminishes or eventually disappears when adding parental information. The last option would, of course, mean that the distinct types of early school-towork trajectories identified merely reflect differences in the young persons' family background. Likewise, the first option would point to the opposite: no link whatsoever between early school-to-work trajectories and family background. Taken together, all this new evidence for the four Nordic countries under study should provide policy-makers valuable information to guide them in their decisions on actions aimed to enhance the transition of youth from school into working life.

\section{Structure and content of the present report}

The report is structured as follows. The next chapter provides a snapshot of what widely used international indicators can tell us about educational outcomes, school-to-work transitions and labour market performance of young people in the Nordic countries. Simultaneously, the information mediated by these mainly static indicators serves as a benchmark when we in the next chapters turn to presenting results obtained based on our national longitudinal data.

Chapter 2 introduces the national datasets underlying the empirical evidence reported in this volume. It also describes and compares across countries the main post-compulsory-school activities in which the young people covered by our data have been engaged in, with the emphasis being on their situation up to age 20. In other words, this chapter paints a general picture of young people's activities over a time period which is often argued to be the most critical in terms of their future labour market outcomes. The chapter also introduces our preferred definition of young people lacking an upper secondary certificate: these young people are called "non-completers", as compared to "completers", implying that they only have their compulsory-school exam still five years after having left primary education.

In the next chapter, Chapter 3 , we present a first set of results obtained by "grouping" the multitude of individual school-to-worktransition pathways followed by young people when aged 16 to 20 , as reported in Chapter 2, by use of so-called cluster analysis. Accordingly, the chapter starts with a brief outline and discussion of the basic idea of the cluster analysis method. Only then we turn to the cluster results produced for each country using information on the full youth popula- 
tion. For comparative purposes, separate results are presented also for the non-completers. All these results on "typical" early school-to-worktransition patterns are obtained by allowing each national dataset to form the clusters for the country in question, for which reason we refer to them as "country-specific cluster results". In other words, we do not restrict the cluster analysis in order to produce as similar clusters as possible across the four Nordic countries under study.

The country-specific cluster results reported in Chapter 3 are used as key inputs for the cluster analyses undertaken in the next two chapters, where we explicitly aim at improving the comparability of early schoolto-work trajectories across the four Nordic countries by identifying what we have labelled "common" Nordic school-to-work trajectories (Chapter 4) and "stylized" school-to-work pathways for the Nordic countries (Chapter 5). For the cluster analysis undertaken in Chapter 4 we have for each country made a list of the observed trajectories (sequences of activities) from age 16 up to age 20 and then calculated the number of young people following each of these sequences. This country-specific information is then pooled into one big data to which we apply cluster analysis in order to allocate all these trajectories of Nordic youth across ten clusters "common" for the four Nordic countries under study. ${ }^{1}$ This approach allows interesting patterns common to the Nordic countries to be identified. Also this analysis is undertaken separately for all young people and non-completers.

In Chapter 5, the focus is on comparing a set of "stylized" school-towork pathways constructed for the four Nordic countries under study. In brief, the basic idea is to first select a number of "typical" early schoolto-work trajectories based on the results obtained in Chapter 3, and then allocate the trajectories of all other young people across these "typical" pathways. By using identical "typical" early school-to-work trajectories as the point of departure for all four Nordic countries, we are able to shed further light on both similarities and dissimilarities across the four countries in relation to young people's post-compulsory-school experiences up to age 20. However, in contrast to the analyses reported in the previous chapters, Chapter 5 is restricted to non-completers only, that is,

\footnotetext{
${ }^{1}$ Hence, for producing the common Nordic cluster results reported in Chapter 4, we have not merged individual-level data for the four Nordic countries under study. Instead, we have merely combined information on individual trajectories showing sequences of activities from age 16 up to age 20. We have asked for and also received permission from the respective statistical bureaus to undertake such a pooling of countryspecific individual trajectories.
} 
to those young people who have no degree beyond the compulsoryschool exam still five years after leaving basic education. A main reason for this choice of focus is that the group of young completers is highly similar in the four countries, whereas we see both striking similarities and distinct differences between the four Nordic countries when it comes to non-completers. By complementing the results produced for non-completers in the previous chapters with information provided by these "stylized" school-to-work pathways, we obtain a fuller picture of the multitude of early post-compulsory trajectories followed by Nordic non-completers.

After Chapter 5, our focus turns from exploring young people's main activities and school-to-work-transition patterns over the five years following upon compulsory education (i.e., from age 16 up to age 20) to investigating what happens to these youngsters after they have turned 20. What kind of main activities - studying, employment, unemployment, disability arrangements or other types of inactivity - are they mostly engaged in as young adults? Can we observe distinct and rather stable differences in this respect across genders, on the one hand, and between those differing in their educational background, on the other hand? Or is it possible that these later outcomes are, by and large, quite similar for young men and women, as well as for early and later completers of an upper secondary degree and, possibly, even for adult noncompleters, i.e. those with no exam beyond primary education still in adulthood? Can we identify conspicuous variations across cohorts obviously related to fluctuations in the economic environment, or is the eventual impact of economic shocks rather outweighed by other processes and mechanisms affecting the labour market situation of young people representing different cohorts? Last, but not least, can we identify clear-cut similarities or dissimilarities in all these important dimensions across the four Nordic countries under study? Chapter 6 sets out to provide answers to these key questions.

While Chapter 6 gives a description of young people's labour market situation in adulthood, Chapter 7 looks into the background of these young people in an attempt to identify factors that seem to be especially strongly related to the labour market outcomes observed up to age 31 . Our statistical analysis relies on so-called multinomial logit models which show the probability of belonging to one of several mutually exclusive groups, given a particular set of background characteristics. In our case, these groups are made up of the five main categories of labour market activities used in the previous chapters: full-time student, employed, unemployed, disability beneficiary or outside all of 
these activities ("other"). The background factors accounted for in these multinomial logit models can be divided basically into two groups: one reflecting family background and the other early schoolto-work-transition patterns, that is, trajectories followed straight after completion of compulsory education up to age 20. Additionally, we account for gender as well as cohort. Accounting for cohort is relevant as we base our analysis on the pooled information available for all three youth cohorts under scrutiny, i.e., those young people who turned 16 in 1993, 1998 and 2003, respectively.

The last chapter of this report, Chapter 8, gathers and discusses the main findings presented in the previous chapters. While each chapter presenting our results, that is, Chapters 3 to 8, contains several sections titled "Main findings" - one for each sub-chapter - this concluding chapter aims to draw, based on our multifaceted results, a broader picture for the four Nordic countries under study. In other words, in this concluding chapter we choose to overlook the multitude of more detailed findings reported and discussed in the separate chapters and sub-chapters. This does by no means imply that these details are trivial. Instead, this way of presenting the main results of our study is the product of a deliberate choice with overall conclusions and remarks given in Chapter 8, with theme-specific conclusions drawn together in the sections titled "Main findings", and detailed country-specific as well as cross-country results and conclusions reported and discussed throughout the text. 



\section{Performance of young people - an international perspective}

The main focus of this chapter is on exploring what internationally widely-used indicators can tell us about educational outcomes, school-towork transitions and labour market performance of young people in the Nordic countries. Simultaneously, the information mediated by these overwhelmingly static indicators serves as an important benchmark when we, starting in the next chapter, turn to presenting results obtained based on our national longitudinal datasets.

\subsection{Setting the stage}

The youth population has been the most affected by the recent global crisis. There are several obvious reasons why young people have been hit so hard (see e.g. OECD, 2009, 2010a; Scarpetta et al., 2010). Schoolleavers are often the first to encounter difficulties: when the labour market deteriorates, employers shed workers and also become much more selective in their hiring of new staff. As those making the transition from school to work compete with more experienced workers for (fewer) jobs, they often face virtually impossible barriers when trying to get a foothold in the labour market. However, the crisis has posed challenges also to those youth who were already in the labour market but holding temporary jobs and/or working in business-cycle sensitive industries; they have often been among the first to lose their jobs. And with the labour market having become more selective, the risk of unemployment for recent entrants is notably higher among those lacking relevant skills or experience and, conversely, they also face particular difficulties in finding a new job.

The relatively higher vulnerability of youth to unemployment and inactivity was, in effect, a widely recognised problem in many European countries even before the onset of the economic crisis. Particular attention was thereby paid to the multiple barriers in finding work 
faced by low-skilled youth, that is, early school-leavers. Indeed, many European economies were already before the crisis tackling a number of labour market problems - judged to affect adversely the transition from school to work of youth as well as their initial labour market experiences - in order to cope with unacceptably high youth unemployment and inactivity rates.

The recent economic crisis is commonly seen to have aggravated many of these structural problems and, consequently, the situation of especially those youth whose labour market prospects were weak already prior to the crisis. The Nordic countries are no exception to this pattern, as shown in statistics compiled by notably Eurostat, ILO and OECD. In particular, although there were significant pre-crisis differences both in the level and evolution of youth unemployment also across the Nordic countries, they are nonetheless characterised by two distinct features which they share with the rest of Europe.

First, youth face a clearly higher risk of unemployment than adults also in Northern Europe. However, while the youth/adult unemployment ratio (for 2008) falls within the interval 2 to 3 for most OECD countries, it ranges between 3 and 4 in seven countries, three of which are located in the northern part of Europe (Denmark, Finland and Norway), and settles above 4 in only two countries - Iceland and Sweden (Scarpetta et al., 2010, pp. 11-12). Second, all Nordic countries have experienced a marked increase in youth unemployment since the recession began.

Increasing youth unemployment rates in combination with discouraging estimates of the likely short-term evolution of youth unemployment soon triggered, in individual countries, a multitude of actions aimed at cushioning the effects of the crisis on youth while, simultaneously, pushing forward the long-term agenda of necessary structural reforms for tackling pre-crisis youth unemployment problems. A major challenge has thereby been to devise short-term measures which do not conflict with but, preferably, complement and support the long-term reform agenda of promoting more and better jobs for youth in response to projected demographic changes.

The short- and long-term measures planned and realised in individual countries have been surrounded by a myriad of activities initiated, not least, by the European Commission and the OECD. The many initiatives of the European Commission were brought to a head in the Europe 2020 framework launched in March 2010 [COM(2010)2020] as a continuation of the Lisbon process. This EU strategy for smart, sustainable and inclusive growth has a strong youth dimension, as have several of its accompanying flagship initiatives, most notably "Youth on the Move" 
[COM(2010)477] and "An Agenda for New Skills and Jobs" [COM(2010)682], but also the Horizon 2020 financial instrument aimed at securing Europe's global competitiveness. Illustrative examples of recent actions supplementing the Youth on the Move education and employment initiative include an action plan to reduce early school leaving in the EU [COM(2011)18, $\operatorname{COM}(2011) 19, \operatorname{SEC}(2011) 96]$, and the Youth Opportunities Initiative [COM(2011)933] launched in December 2011, which can be described as a set of measures, planned for 2012 and 2013, to drive down youth unemployment. The Youth Employment Package adopted in December 2012 can be seen as a key milestone of this YOI. Most notably, this package of Commission proposals to fight youth unemployment recommended that member states introduce a Youth Guarantee, launch a consultation of European social partners on a Quality Framework for Traineeships, and announce a European Alliance for Apprenticeships. The EU countries endorsed the principle of the Youth Guarantee in April 2013. Until the end of 2014, the EU provided an advice service on apprenticeship and traineeship schemes in order to support its Member States to develop high quality apprenticeship and traineeship programs.

Complementary to the Youth on the Move flagship initiative of the Europe 2020 strategy is, inter alia, the EU Youth Strategy: Council Resolution on the renewed framework for European cooperation in the youth field (2010-2018) (OJ C 311, 19.12.2009, pp. 1-11). A particular feature of this strategy is that an EU Youth Report is to be drawn up at the end of each three-year cycle to evaluate the progress made towards the overall objectives of the strategy, on the one hand, and to serve as a basis for establishing a set of priorities for the coming work cycle, on the other. The first work-cycle EU Youth Report [COM(2012)495 final] was published in September 2012 and adopted as a Joint Council-Commission Report in November 2012. In relation to the EU Youth Strategy, a dashboard of EU Youth Indicators was released in 2011 [SEC(2011)401]. Key instruments to support the EU Youth Strategy are, most notably, the Lifelong Learning and Youth in Action programs and the Erasmus for All program.

Among the many recent initiatives of the OECD, two in particular deserve to be mentioned here. The first is the High-Level Policy Forum on Jobs for Youth: Addressing Policy Challenges in OECD Countries, which was organised jointly with the Norwegian Ministry of Labour in late September 2010. The main issues and policy recommendations on how to tackle youth unemployment problems presented at this Forum, and later published in a comprehensive report (OECD, 2010b), synthesised the findings of thematic reviews of Jobs for Youth undertaken over the 
years 2006-2009 in 16 member countries. While the review for Norway (OECD, 2008c) took place against the background of a buoyant economy, the corresponding review for Denmark (2010c) was more concerned with youth unemployment in the context of the ongoing economic crisis. These thematic reviews did not cover Finland, Iceland or Sweden, though. This High-Level Policy Forum was preceded, about one week earlier, by a joint ILO-IMF conference - also arranged in Oslo but in cooperation with the Office of the Prime Minister of Norway - on The Challenges of Growth, Employment and Social Cohesion, one focus of which was youth unemployment (ILO-IMF, 2010). A second key initiative of the OECD is the so-called OECD Action Plan for Youth, launched in 2013 as an integral part of OECD's work on youth, and the high-level meetings and comprehensive reports related to the implementation of the plan (http://www.oecd.org/employment/action-plan-youth.htm).

\subsection{Snapshot of recent Nordic comparative studies}

The Nordic Council of Ministers has also in recent years initiated several reports on how the youth unemployment problem is addressed in the Nordic countries. Especially the following reports should be mentioned in this context. A report edited by Markussen (2010) focuses on dropout in upper secondary education in the Nordic countries. In particular, for each Nordic country it provides an overview of the structure of upper secondary education, looks at research and results on dropouts, gives an overview of implemented measures to reduce dropout and improve through-put of students and, finally, assesses possible effects of the implemented measures. It concludes by pointing to the need for further reforms to reduce dropout and improve levels of upper secondary completion. Another report, by Engberg (2014), continues on this topic in the sense that it describes the reforms and other actions carried out in the Nordic countries concerning vocational and apprenticeship training, and also reflects on the challenges characterising these systems, using desk studies and interviews with national experts as analytical tools. Still another report of some relevance in this context describes both existing and planned measures, as initiated by relevant government departments, to prevent youth unemployment in the Nordic countries (Ramböll Management Consulting AB, 2010). The report reviews both short-term measures implemented during the economic crisis and longterm measures related to future demographic challenges. This report 
also relies on desk studies and interviews with national experts and public servants within the fields of education and labour markets.

While this kind of comparative analysis of short- and long-term measures can provide important information on what is done and planned - and in relation to which target group(s) and with which expected outcomes in mind - in different countries, they can tell little, if anything, about the genuine impact of the measures undertaken. Such information can be obtained only by means of carefully designed and performed evaluation studies. However, the international research focusing on in-depth evaluation of various modes of measures directed at disadvantaged and/or unemployed youth is still surprisingly scant. More important, the evidence produced by such evaluations has, so far, been rather discouraging when it comes to both impact and effectiveness [see e.g. the reviews by Asplund (2009) and Asplund and Koistinen (2014) and, especially, the references therein].

Also worth mentioning in this context is a recent report by Halvorsen et al. (2012) on the transition between school and work for particularly vulnerable groups of youth. It concludes that between $2 \%$ and $5 \%$ of the youth cohorts are already "outsiders". The report summarises the existing knowledge on vulnerable youth, discusses challenges and policy measures in the Nordic countries, with particular attention paid to youth, and suggests the building of a Nordic knowledge bank for good practices.

\subsection{The Nordic story told by international comparative indicators}

Another source highlighting the youth unemployment problem from a cross-country perspective contains indicators developed and compiled mainly by Eurostat and OECD, and published on a regular basis. Excellent examples are Eurostat's statistical portraits of youth in Europe and the OECD's Education at a Glance reports. In this sub-chapter, we draw on some of these statistical sources to provide an overview of recent developments in the Nordic countries, also in comparison with the rest of Europe and the OECD area.

While offering comprehensive information, these sources also have their shortcomings. First, the provided information is basically static in nature in the sense that it gives a snapshot of the situation in - typically a specific year, and compares the findings with corresponding information from a previous year (given that such information is available). Accordingly, this type of year-specific information highlights the average situation at 
a given point in time, but can say nothing about the underlying dynamics such as the shifts over time of young people between education, work, unemployment and inactivity. Second, indicators aiming to describe at least some specific dynamic aspect of this continuous transition process can usually be derived only for a limited number of countries with the Nordic countries mostly being surprisingly weakly represented.

This is the prevailing situation also in specific studies, of which Quintini and Manfredi (2009) is an illustrative example. They address a topical issue - the dynamic nature of youth labour market situations and key pathways of youth leaving secondary education - but, due to data limitations, they can only include Denmark and Finland out of the Nordic countries and only up to the year 2001. Simultaneously, this highlights the lack of extensive cross-country comparative evidence on the dynamic nature of the labour market situation of youth in the Nordic countries and the key pathways followed by school-leaving youth having acquired different levels of education - not merely a secondary diploma as in the Quintini and Manfredi (2009) study. Indeed, this is the kind of analysis that we will undertake in the subsequent chapters of this report.

A key challenge when addressing the issue of young people's unemployment problems and risks of marginalisation is that the youth population is far from homogenous. School-leavers are equipped with different quantities and qualities of formal education. They also differ in a multitude of other dimensions, including school experiences and early lifetime experiences, notably family background. Their transition from school to work and initial labour market experiences reveal considerable variation both in length and quality. This variation has been shown to partly originate in differences in the school-leavers' educational and social background (e.g. Markussen, 2010). However, it has also been maintained to largely reflect the functioning of the labour market, that is, the labour market institutions in force and the labour market policies pursued in a rapidly changing economic and social environment. Indeed, several reports have shown that the conditions for young people to establish themselves in the labour market reveal important differences also across the Nordic countries (see e.g. Olofsson and Wadensjö, 2007; Olofsson and Panican, 2008).

Furthermore, while early unemployment is known to affect the youth to a substantial degree, it is also recognized that the short- and longterm consequences of early unemployment differ markedly across young individuals. As pointed out in the outset, a growing body of literature - with evidence emerging also for the Nordic countries - indicates that spells of unemployment entail the risk of creating permanent scars 
especially for disadvantaged youth who tend to be particularly illprepared for today's labour market.

Our subsequent comparisons, based on available international data, clearly illustrate how important it is to distinguish between young people's different situations and composites of activities also when undertaking international comparisons. In particular, since young people often combine school with part-time work, especially in the Nordic countries, the results obtained vary substantially with the way in which the status of youth is measured. Let us start by first reflecting on youth employment.

\subsubsection{Youth employment}

Our national longitudinal datasets cover three full youth cohorts: all young people turning 16 in 1993, 1998 or 2003 (see Chapter 2). We therefore start by illustrating - using OECD statistics - the overall employment situation for young people five years later, that is, when our young people turned 21. By this age, most of them could be expected to have completed an upper secondary degree. What actually happened to the young people contained in our three youth cohorts will become evident in the next chapter.

Table 1.1 presents OECD employment population ratios for the Nordic countries for four selected years: in 1998, 2003 and 2008, as well as in the last year of available data, 2013. In all these years, Denmark shows up with the highest employment population ratio, both for the very young and for the 20-24 year-olds, followed by Norway, Finland and Sweden. However, the table also reveals that Denmark experienced a marked decline in employment population ratios up to 2003, that Finland saw a clear improvement in employment population ratios between 2003 and 2008, and that the employment population ratios of Norwegian males deteriorated in 2003 but recovered by 2008, whereas the employment population ratios of Swedish males started increasing already in 1998. In all four countries, there was a remarkable drop in the employment population ratios of young people between 2008 and 2013. This decline was notably stronger for young men than for young women. 
Table 1.1: Employment population ratios (\%) for Nordic youth in 1998, 2003, 2008 and 2013

\begin{tabular}{|c|c|c|c|c|c|c|c|}
\hline \multirow{2}{*}{\multicolumn{2}{|c|}{$15-19$ year-olds }} & \multirow[t]{2}{*}{1998} & \multirow[t]{2}{*}{2003} & \multirow[t]{2}{*}{2008} & \multirow[t]{2}{*}{2013} & \multicolumn{2}{|c|}{ 2008-2013 } \\
\hline & & & & & & \%-points & $\%$ \\
\hline Denmark & males & 57.9 & 51.4 & 59.2 & 41.1 & -18.1 & -30.6 \\
\hline Denmark & females & 60.2 & 49.5 & 59.1 & 47.1 & -12.0 & -20.3 \\
\hline Finland & males & 25.7 & 24.7 & 26.6 & 17.0 & -9.6 & -36.1 \\
\hline Finland & females & 19.9 & 23.3 & 27.5 & 26.6 & -0.9 & -3.3 \\
\hline Norway & males & 42.2 & 38.4 & 42.1 & 33.8 & -8.3 & -19.7 \\
\hline Norway & females & 42.3 & 43.0 & 46.1 & 39.5 & -6.6 & -14.3 \\
\hline Sweden & males & 21.7 & 25.8 & 20.3 & 17.5 & -2.8 & -13.8 \\
\hline Sweden & females & 25.1 & 32.2 & 26.0 & 24.1 & -1.9 & -7.3 \\
\hline \multicolumn{8}{|c|}{ 20-24 year-olds } \\
\hline Denmark & males & 75.6 & 71.9 & 76.3 & 63.4 & -12.9 & -16.9 \\
\hline Denmark & females & 71.2 & 65.4 & 71.9 & 62.6 & -9.3 & -12.9 \\
\hline Finland & males & 60.2 & 61.8 & 70.1 & 54.5 & -15.6 & -22.3 \\
\hline Finland & females & 48.7 & 54.7 & 62.3 & 60.5 & -1.8 & -2.9 \\
\hline Norway & males & 74.3 & 68.8 & 74.8 & 67.4 & -7.4 & -9.9 \\
\hline Norway & females & 65.0 & 65.2 & 71.5 & 67.6 & -3.9 & -5.5 \\
\hline Sweden & males & 58.2 & 63.1 & 66.8 & 59.4 & -7.4 & -11.1 \\
\hline Sweden & females & 52.5 & 57.3 & 59.8 & 58.0 & -1.8 & -3.0 \\
\hline
\end{tabular}

Note: The employment population ratio measures the employed as a percentage of the population in the age group.

Source: OECD Labour force statistics. OECDiLibrary.

Figure 1.1 extends the picture by showing employment population ratios of youth aged 15-24 for the Nordic countries in comparison with selected large OECD economies (with the information referring to the 1st quarter of 2012). The employment population ratios displayed in the figure are measured in two different ways: the vertical axis gives the employment population ratio for all young people, whereas the horizontal axis restricts the employment ratio to non-students only, as youth employment ratios can be criticised for being blurred by pupils and students working part-time while in school. This setting reveals that Denmark, Iceland and Norway have high youth employment ratios, together with Germany, the UK and USA, also when account is made for part-time working students. Finland and Sweden, on the other hand, move from above the OECD average to below the OECD average when the youth employment ratio is adjusted with respect to students working on a part-time basis. 
Figure 1.1: Employment population ratios (\%) for all young people aged 15-24 (vertical axis) and separately for non-students only (horizontal axis), 2012 (1st quarter)

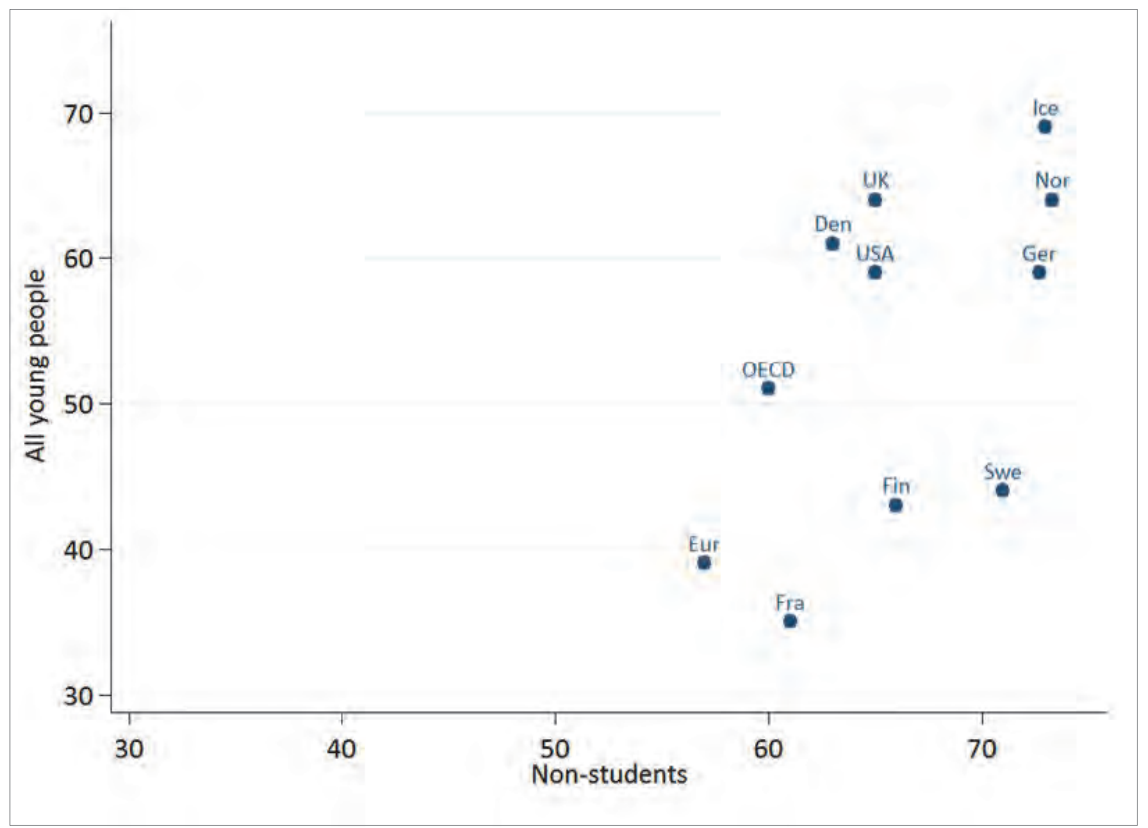

Note: The employment population ratio measures the employed as a percentage of the population in the age group.

Source: OECD based on national Labour Force Surveys.

\subsubsection{Youth unemployment}

Unemployment is a much more widespread phenomenon among youth than among adults virtually everywhere. Young people are also the first to be hit by rising unemployment, and typically the last to benefit from recovering labour markets. Table 1.2 shows unemployment rates, measured as a percentage of the labour force, for the four Nordic countries under study in the years 1998, 2003, 2008 and 2013. Among young people under age 20, Denmark stands out with a comparatively low unemployment rate in 1998, whereas Finland was still struggling with high post-recession youth unemployment. The unemployment rate of the very young has been increasing in all four countries from 1998 to 2013, except for Norway where it has declined, but only among young women. The unemployment rates of young people aged 20-24 are also high, but not as dramatically high as for those aged 15-19. Yet, in both Finland and Sweden, about one-fifth of the male labour force of 20-24 year-olds was unemployed in 2013, according to OECD statistics. 
Table 1.2: Unemployment rates (\%) for Nordic youth in 1998, 2003, 2008 and 2013

\begin{tabular}{|c|c|c|c|c|c|c|c|}
\hline \multirow{2}{*}{\multicolumn{2}{|c|}{$15-19$ year-olds }} & \multirow[t]{2}{*}{1998} & \multirow[t]{2}{*}{2003} & \multirow[t]{2}{*}{2008} & \multirow[t]{2}{*}{2013} & \multicolumn{2}{|c|}{$2008-2013$} \\
\hline & & & & & & \%-points & $\%$ \\
\hline Denmark & males & 8.0 & 9.9 & 7.9 & 17.9 & 10.0 & 126.6 \\
\hline Denmark & females & 9.3 & 10.7 & 10.9 & 13.9 & 3.0 & 27.5 \\
\hline Finland & males & 24.6 & 25.9 & 23.3 & 34.1 & 10.8 & 46.4 \\
\hline Finland & females & 33.3 & 31.5 & 25.4 & 27.6 & 2.2 & 8.7 \\
\hline Norway & males & 13.2 & 17.3 & 13.2 & 13.3 & 0.1 & 0.8 \\
\hline Norway & females & 13.7 & 14.8 & 10.5 & 11.3 & 0.8 & 7.6 \\
\hline Sweden & males & 21.1 & 18.6 & 32.5 & 39.3 & 6.8 & 20.9 \\
\hline Sweden & females & 20.6 & 18.3 & 31.8 & 34.5 & 2.7 & 8.5 \\
\hline \multicolumn{8}{|c|}{$20-24$ year-olds } \\
\hline Denmark & males & 5.7 & 8.6 & 7.0 & 11.8 & 4.8 & 68.6 \\
\hline Denmark & females & 6.4 & 8.1 & 6.6 & 10.3 & 3.7 & 56.1 \\
\hline Finland & males & 18.0 & 16.7 & 11.5 & 19.2 & 7.7 & 67.0 \\
\hline Finland & females & 20.0 & 16.3 & 11.4 & 11.7 & 0.3 & 2.6 \\
\hline Norway & males & 7.0 & 10.4 & 4.9 & 9.2 & 4.3 & 87.8 \\
\hline Norway & females & 7.1 & 8.3 & 4.1 & 5.6 & 1.5 & 36.6 \\
\hline Sweden & males & 16.2 & 12.6 & 13.9 & 20.1 & 6.2 & 44.6 \\
\hline Sweden & females & 14.3 & 9.9 & 14.0 & 17.0 & 3.0 & 21.4 \\
\hline
\end{tabular}

Notes: The unemployment rate measures the number of unemployed as a percentage of the labour force in the age group. Note also that Sweden undertook, in 2007, a harmonisation of the unemployment definition towards the one used by ILO. This harmonisation seems to be overlooked in the OECD statistics, implying that the Swedish figures for 1998 and 2003 are not directly comparable with those for 2008 and onwards. Based on harmonised data, the unemployment rate of 15-19 year-old males and females was in $200327.1 \%$ and $23.8 \%$, respectively. These rates notably exceed those given above. The discrepancy is much lower for 20-24 year-olds: an unemployment rate of $14.8 \%$ for men and $12.5 \%$ for women according to harmonised data published by the Swedish statistical bureau SCB.

Source: OECD Labour market statistics. OECDiLibrary.

The unemployment rates reported in Table 1.2 are commonly used in international comparisons of youth unemployment. They are based on Labour Force Survey (LFS) data and measured in a traditional way: by relating the number of unemployed to the total labour force (employed + unemployed). This ILO-LFS measure of unemployment is based on questions in the LSF asking the interviewees, inter alia, whether they have been actively looking for a job during the past four weeks. The LFS measure of the total number of unemployed may, as a consequence, also include young people who are actively looking for a job while also being enrolled as full-time students.

Another way of measuring unemployment is to rely on registered unemployment, which merely comprises those who have registered as unemployed jobseekers at an unemployment office (PES). The extent to which registered unemployment includes full-time students depends on the eligibility conditions of young people for registering as unemployed jobseekers. Table 1.3, which is reproduced from Halvorsen et al. (2012), shows the percentage share of youth in each age group registered as 
unemployed in the years 2000 and 2005-2009. We note that the difference between registered unemployment and LFS unemployment is particularly large for young people, especially for Sweden. A major reason for this discrepancy is that registered unemployment is typically lower among young people not eligible for unemployment benefits.

Table 1.3: Registered unemployment rates (\%) for Nordic youth, 2000 and 2005-2009

\begin{tabular}{lllllll} 
Age & $\mathbf{2 0 0 0}$ & $\mathbf{2 0 0 5}$ & $\mathbf{2 0 0 6}$ & $\mathbf{2 0 0 7}$ & $\mathbf{2 0 0 8}$ & $\mathbf{2 0 0 9}$ \\
Denmark & & & & & \\
$16-19$ & & 1.0 & 0.8 & 0.7 & 0.7 & 1.0 \\
$20-24$ & & 3.7 & 3.7 & 2.7 & 2.5 & 4.7 \\
$16-64$ & & 4.3 & 2.9 & 2.1 & 3.6 \\
Finland & & & & & \\
$16-19$ & 3.2 & 2.5 & 2.4 & 2.4 & 2.7 & 3.5 \\
$20-24$ & 9.4 & 9.0 & 8.6 & 8.1 & 8.4 & 10.9 \\
Iceland & & & & & \\
$16-19$ & 0.7 & 0.9 & 0.5 & 0.4 & 0.9 & 3.6 \\
$20-24$ & 1.1 & 2.4 & 1.4 & 1.0 & 2.1 & 9.9 \\
$16-70$ & 1.1 & 1.7 & 1.1 & 0.5 & 1.4 & 6.8 \\
Norway & & & & & & \\
$16-19$ & 1.2 & 1.2 & 1.0 & 0.7 & 0.7 & 1.0 \\
$20-24$ & 3.3 & 4.5 & 3.2 & 2.2 & 2.0 & 3.5 \\
$16-74$ & 2.7 & 3.5 & 2.6 & 1.9 & 1.7 & 2.7 \\
Sweden & & & & & & \\
$16-19$ & 1.5 & 2.2 & 1.8 & 1.6 & 1.8 & 2.9 \\
$20-24$ & 5.0 & 5.7 & 5.9 & 5.0 & 4.0 & 6.0 \\
$16-64$ & 4.1 & 4.2 & 3.6 & 2.9 & 2.8 & 4.0 \\
\hline
\end{tabular}

Notes: The unemployment rate measures the number of registered unemployed as a percentage of the population in the age group. Information for the year 2000 is missing for Denmark while information on all age groups combined is not available for Finland.

Source: Halvorsen et al. (2012).

Also the information on unemployment contained in our national longitudinal datasets used in the subsequent chapters refers to registered unemployment. However, it differs from the information underlying the registered unemployment reported in Table 1.3, as our datasets are recoded to identify all young people enrolled in education as full-time students also when they appear as employed or unemployed in the original data (see further Chapter 2). Indeed, as in the case of youth employment, the way full-time students are treated makes a big difference also when calculating youth unemployment rates. We illustrate this by going back to the LFS data to show what happens to the ranking of the Nordic countries depending on the unemployment measure used.

We thereby start by showing, in Figure 1.2, the number of students as a percentage share of the population in the age group. In the LFS data, more than $80 \%$ of those aged $16-19$ report studying to be their main activity. For those aged 20-24, this share is notably lower, ranging from 
40 to almost $60 \%$ with Denmark having the highest and Sweden the lowest share of young people reporting studying as their main activity. In view of these large shares of students in both youth populations, it is obvious that the results obtained depend critically on the way students are treated in the calculations.

Figure 1.2: Students as a share (\%) of the population in the age group, for four Nordic countries

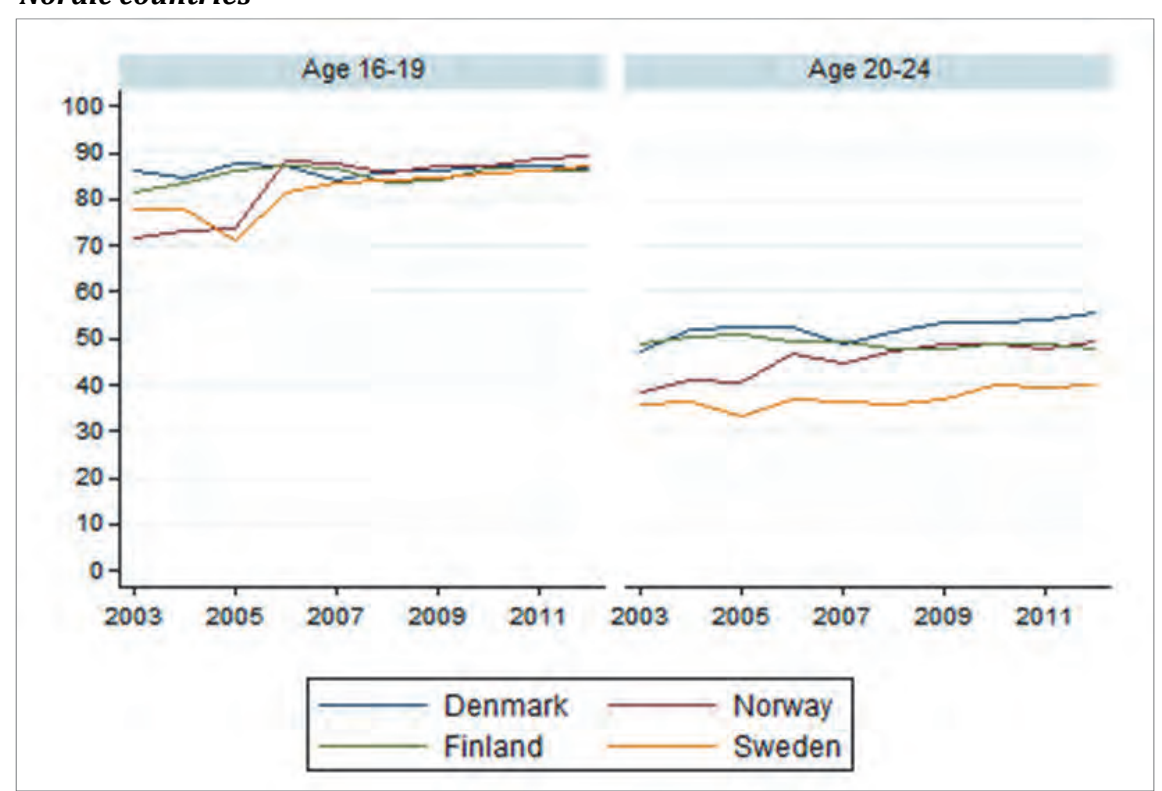

Note: Students are defined as young people who report studying to be their main activity in the LFS. The drops in the series in $\mathbf{2 0 0 5}$ for Norway and Sweden are most likely due to data issues. Cf. the notes in Table 1.2.

Source: Own calculations based on LFS quarterly data.

Let us now return to unemployment. Figure 1.3 provides information on youth unemployment from the 1st quarter of 2012 for the Nordic countries as well as for a selected number of non-Nordic countries. The horizontal axis measures the unemployment rate, that is, the number of unemployed as a percentage of the labour force. Sweden comes out with a higher youth unemployment rate than the average for Euro countries (EURO). Sweden, together with Finland, also ranks higher than both the UK and the USA.

The vertical axis, in turn, measures youth unemployment by means of the unemployment ratio, that is, the number of unemployed as a percentage of the whole youth population. While 23\% of the European youth labour force is unemployed, these unemployed young people constitute less than $10 \%$ of the youth population. In terms of the unem- 
ployment ratio, however, all Nordic countries except Norway score higher than the average for Euro countries. In both Iceland and Sweden, youth unemployment is, in fact, higher than in Italy when measured by means of the unemployment ratio.

Figure 1.3: Youth unemployment rates (\%) and unemployment ratios (\%), the Nordic countries and selected non-Nordic countries, 2012 (1st quarter)

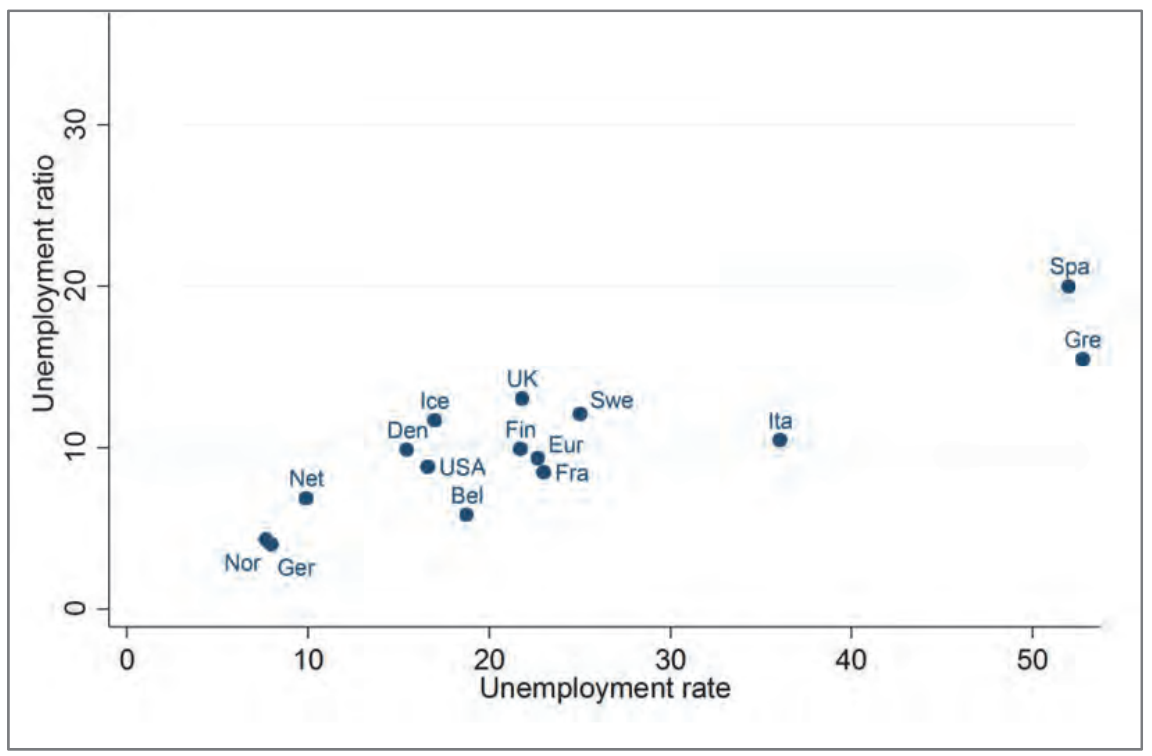

Notes: Unemployment rate = unemployed 15-24 year-olds in relation to the labour force of the age group. Unemployment ratio = unemployed 15-24 year-olds in relation to the whole population of the age group.

Source: OECD as reproduced from Albæk et al. (2014).

However, many of the young people recorded as unemployed in the LFS are actually attending school and, moreover, typically on a full-time basis. Figure 1.4 shows what happens when we remove from the pool of young unemployed those who report studying as their main activity. The vertical axis of Figure 1.4 now shows unemployed young persons who are not attending school. For the Euro area as a whole, the youth unemployment ratio drops from $9.3 \%$ to $7.2 \%$ of the youth population. For the Nordic countries, the change is even larger. After this correction, all Nordic countries rank among those with the lowest level of youth unemployment (among the non-students).

The countries with the largest drop below the 45-degree line added to Figure 1.4 are the ones with the largest proportion of unemployed youth who are also students. The pattern displayed in the figure thus arises from the fact that the unemployed young persons who are not at the same time attending school make up a smaller proportion in the Nordic countries 
compared to the other countries in the figure. In all Nordic countries, the proportion of unemployed young people who are not also in school is less than one-half, and as small as one-third in Sweden.

The huge difference between youth unemployment ratios including and excluding unemployed students is also evident in the next two figures which, moreover, separate 16-19 year-olds from 20-24 year-olds. A comparison of Figure 1.5a with Figure 1.5b reveals a strikingly large drop in the unemployment ratio for the very young (16-19 year-olds) when leaving out students recorded as being unemployed.

Figure 1.4: Youth unemployment ratios (\%) with and without students, the Nordic countries and selected non-Nordic countries, 2012 (1st quarter)

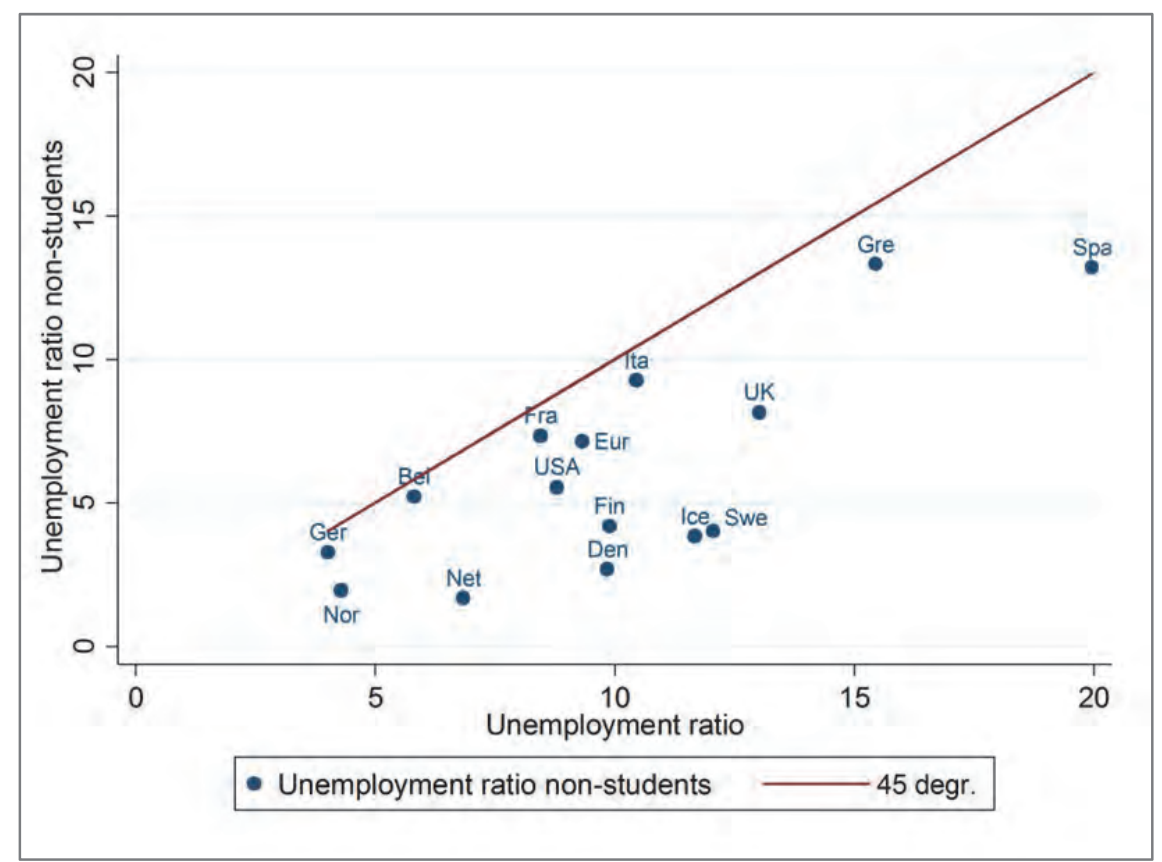

Notes: Unemployment ratio = unemployed 15-24 year-olds in relation to the whole population of the age group. Unemployment ratio, non-students = unemployed 15-24 year-olds with studying not being their main activity, in relation to the whole population of the age group. The red line shows the 45 degree angle.

Source: OECD, as reproduced from Albæk et al. (2014). 
Figure 1.5a: Youth unemployment ratios (\%) for four Nordic countries, 1995-2012

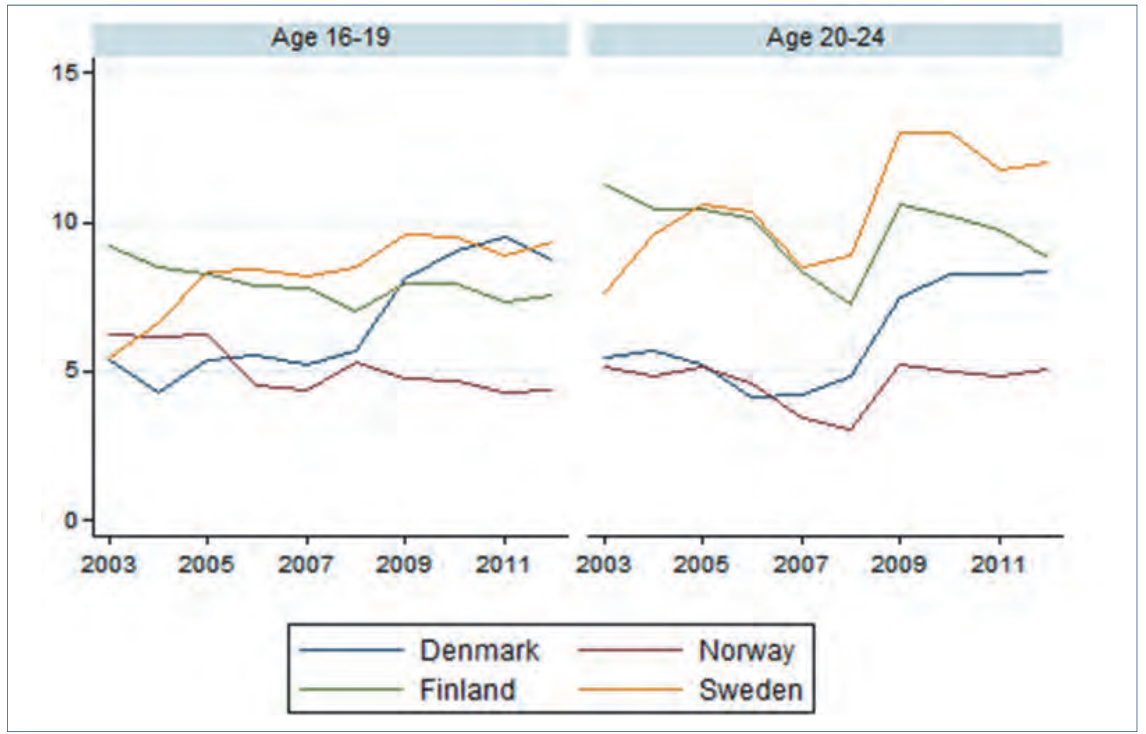

Notes: Unemployment ratio = unemployed in relation to the whole population of the age group. Cf. the notes in Table 1.2 concerning the unemployment information on Sweden prior to 2005. Source: Own calculations based on LFS quarterly data.

Figure 1.5b: Youth unemployment ratios (\%), excluding students, for four Nordic countries, 1995-2012

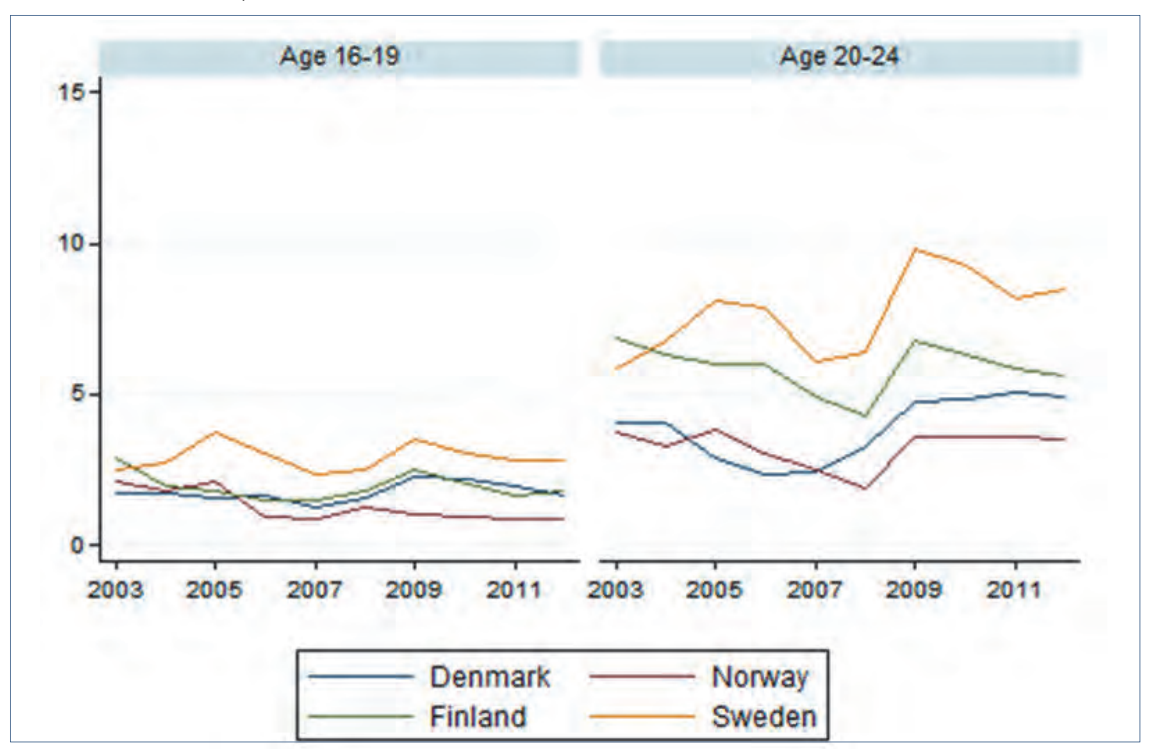

Notes: Unemployment ratio = unemployed in relation to the whole population of the age group. Students are defined by self-reported main activity. Cf. the notes in Table 1.2 concerning the unemployment information on Sweden prior to 2005.

Source: Own calculations based on LFS quarterly data. 


\subsubsection{NEET rates}

NEETs refer to young people who are Not in Employment, Education or Training. Figure 1.6 gives NEET rates for the last quarter of 2012 for 15-24 year-olds in OECD countries, measured as a percentage of the total population in the age group. The Nordic countries are located relatively high up on this ranking scale, implying that they are characterised by low NEET rates. For 2012, the share of young people in NEET activities was estimated to be $5.9 \%$ for Denmark, $6.7 \%$ for Norway, $7.2 \%$ for Sweden and 8.4\% for Finland.

All in all, the information provided so far shows that the labour market is quite accessible for young people in the Nordic countries. In particular, employment rates are relatively high among those who do not attend school and, conversely, unemployment rates are relatively low. The descriptive evidence presented above also implies that the attachment to the labour market is relatively strong also among young people attending school. This follows from the fact that large shares of young people recorded to be employed or unemployed are, actually, pupils and students with studying as their main activity. Simultaneously, they are an integral part of the labour force.

Figure 1.6: NEET rates (\%) for 15-24 year-olds in OECD countries, as split by unemployment and inactivity, 2012 (4th quarter)

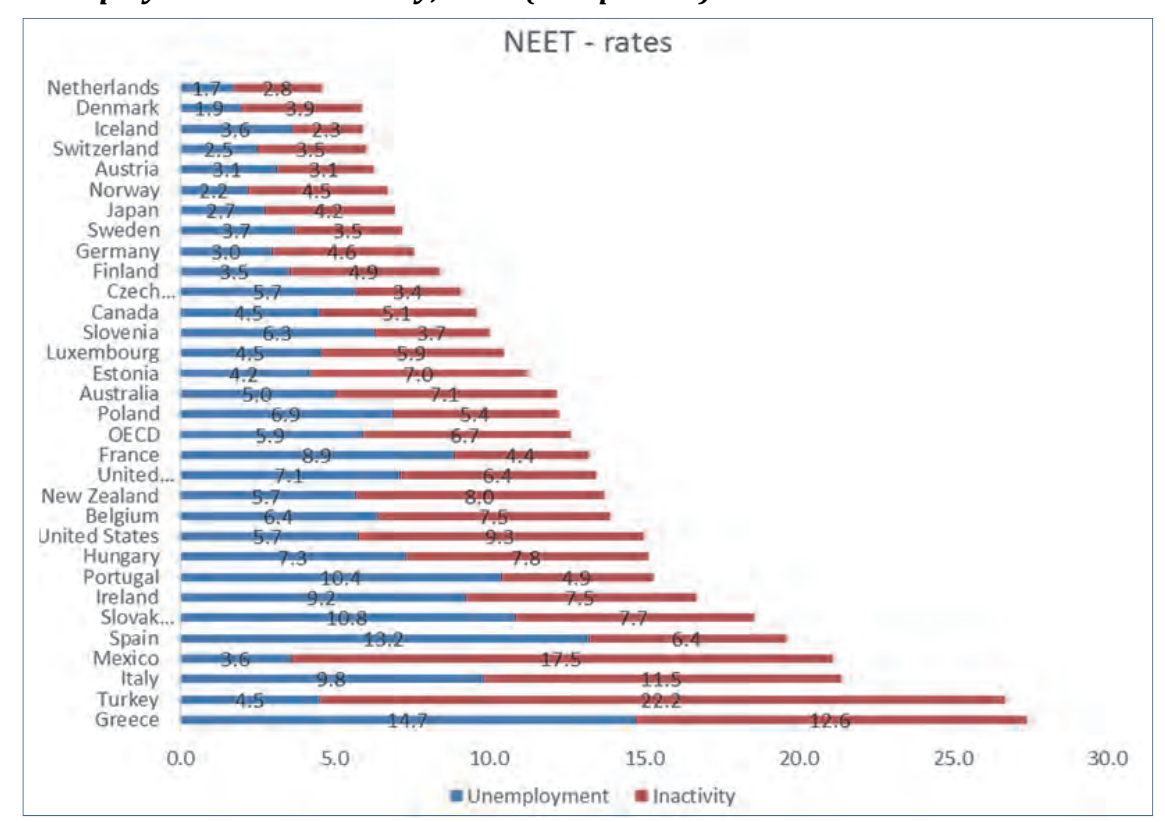

Notes: NEET rate = young people not in employment, education or training as a percentage of the population in the age group.

Source: OECD Society at a Glance 2014. 


\subsubsection{Upper secondary education - completion, non- completion and dropout}

Finally, we take a closer look at school completion, non-completion and dropout with the focus being on upper secondary education. The proportion of 30-34 year-olds holding an upper secondary degree is not particularly high in Denmark or Norway, as is evident in Figure 1.7. Finland and Sweden, on the other hand, rank quite high with more than $90 \%$ of the 30-34 year-olds holding an upper secondary degree in 2012 .

If we explore the average age of upper secondary graduation (Figure 1.8), Sweden comes out with the lowest average age among the Nordic countries, or about 18 years-of-age. In the other Nordic countries, the average graduation age is much higher for both general programs and especially for vocational programs. Indeed, the average age of upper secondary graduation from vocational programs is 28 in both Denmark and Norway. For Finland, it is reported to be even higher.

Figure 1.7: Attainment (\%) of an upper secondary degree among 30-34 yearolds in OECD countries, 2012

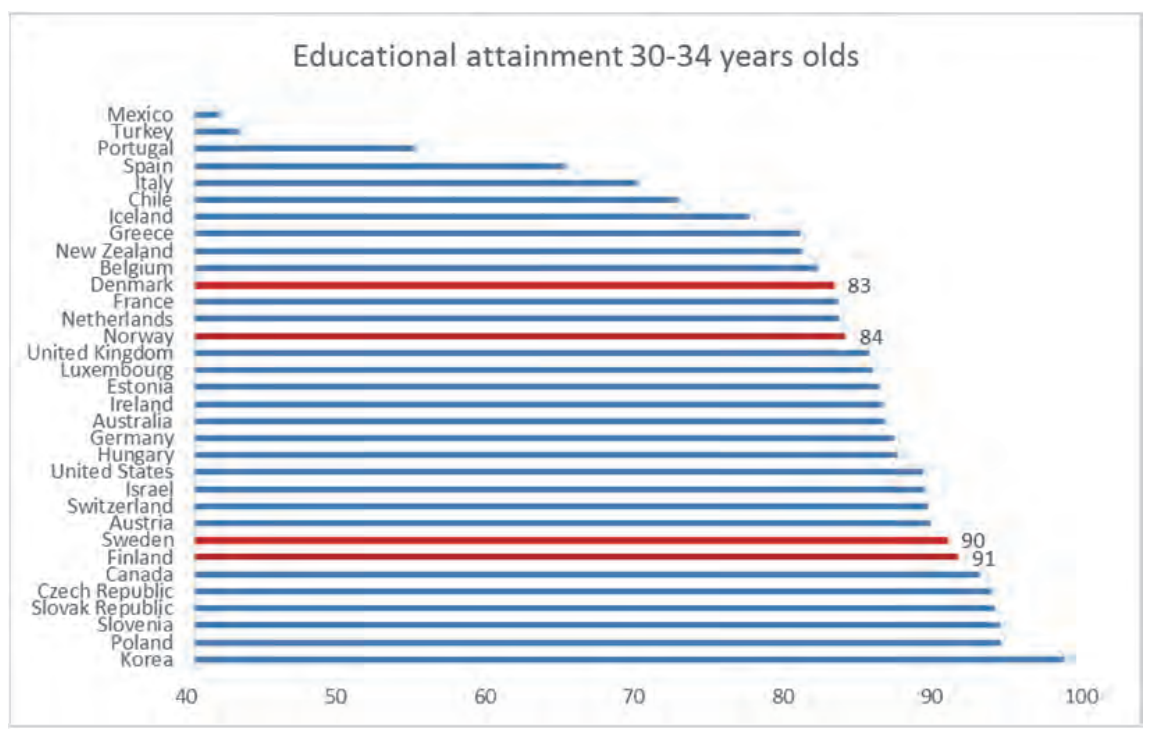

Source: OECD Education at a Glance 2014. 
Figure 1.8: Average age of upper secondary graduation in OECD countries, 2012

Chart A2.2. Average age ${ }^{1}$ of upper secondary graduation (2012)

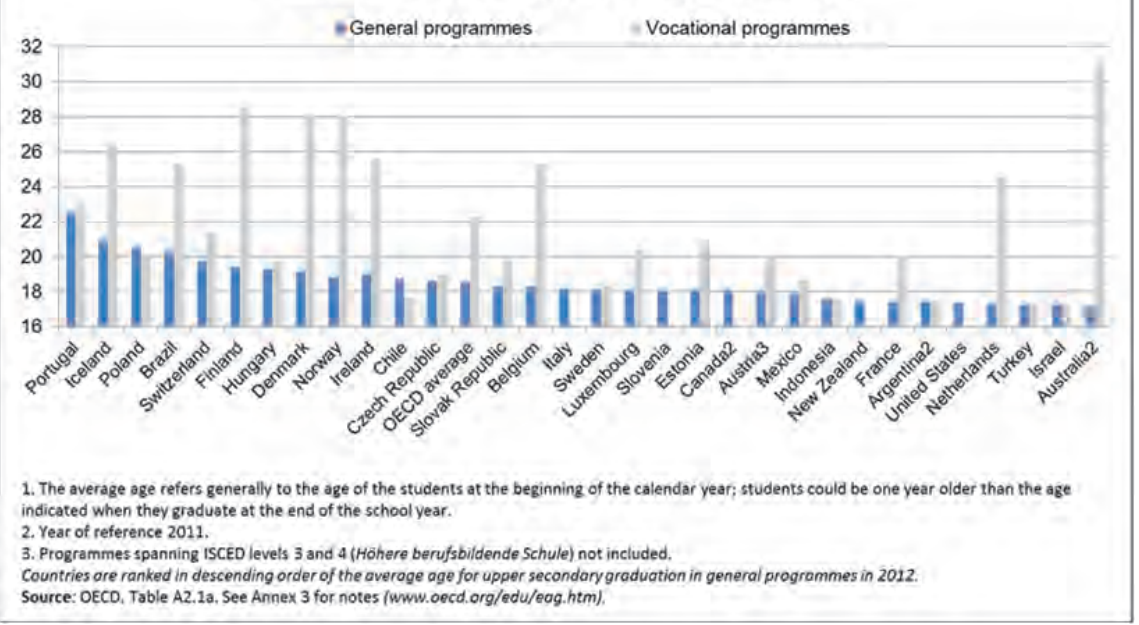

Source: OECD Education at a Glance 2014, Chart A2.2.

There are numerous ways to measure dropout or non-completion rates from upper secondary education. Figure 1.9 illustrates the share of students who successfully completes after the theoretical duration of the upper secondary program, or two years after the theoretical duration of the program. The countries are sorted according to the successful completion of girls in upper secondary programs. All Nordic countries rank below the OECD average, with Sweden and Finland doing slightly better than Denmark and Norway. 
Figure 1.9: Upper secondary completion rates (\%) in OECD countries, by gender

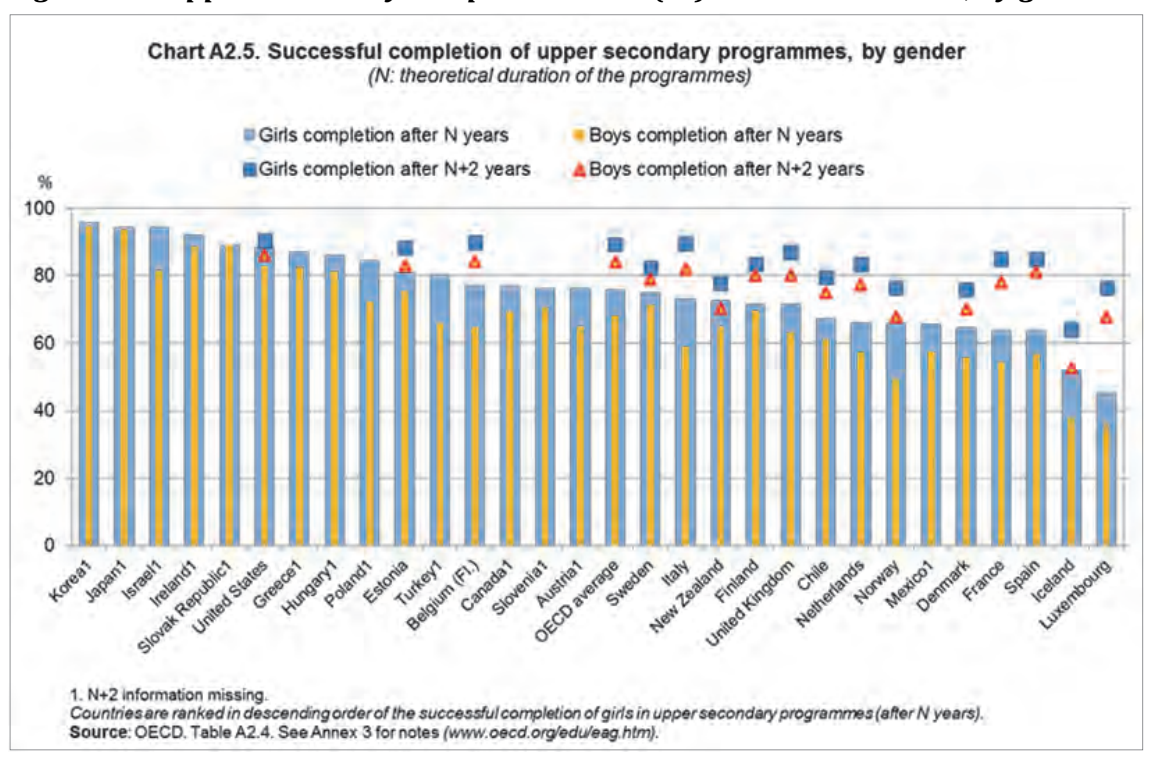

Source: Education at a Glance 2014. Chart A2.5.

Table 1.4, finally, presents alternative measures of completion rates, as reported by Eurostat and OECD. While Figure 1.9 gives the completion rates among those who attend upper secondary programs, Table 1.4 presents completion rates as percentage shares related to the whole youth population.

Table 1.4: Completion, early leaving and dropout rates (\%) for the Nordic countries

\begin{tabular}{|c|c|c|c|c|c|c|}
\hline Upper secondary graduation & & Denmark & Finland & Iceland & Norway & Sweden \\
\hline$<25$ year-olds & 2011 & 79.4 & 85.0 & 70.4 & 77.7 & 75.4 \\
\hline$\geq 25$ year-olds & 2011 & 10.2 & 11.0 & 17.4 & 11.8 & 0 \\
\hline Total & & 89.6 & 96.0 & 87.8 & 89.5 & 75.4 \\
\hline Early school leavers & 2007 & 12.9 & 9.1 & 23.2 & 18.4 & 8.0 \\
\hline (Eurostat) & 2012 & 9.1 & 8.9 & 20.1 & 14.8 & 7.5 \\
\hline $\begin{array}{l}\text { School dropouts } \\
\text { (OECD scoreboard for youth) }\end{array}$ & 2009 & 14.2 & 9.7 & 55.2 & 20.3 & 7.4 \\
\hline
\end{tabular}

Notes: The first lines show expected graduation rates before and after the age of 25 as reported in the OECD publication Education at a Glance. "Early school leavers" (Eurostat) refer to persons aged 18-24 fulfilling the following two conditions: first, the highest level of education or training attained is ISCED 0, 1, 2 or 3c short; second, respondents declared not having received any education or training in the four weeks preceding the survey (numerator). The denominator consists of the total population of the same age group, excluding no answers to the questions "highest level of education or training attained" and "participation in education and training". Both the numerators and the denominators come from the EU LFS (Eurostat). The OECD Scoreboard for youth: share of youth not in education and without an ISCED 3 degree. 
The information contained in Table 1.4 clearly shows that the picture looks quite different depending on both the measure used and the time of measurement. Again, the reason for this is that youth career trajectories are very heterogeneous, involving both multiple states at a given point in time (e.g. combined schooling and employment) and frequent transitions back and forth between different states (e.g. educationemployment-unemployment-education). As we will show in the next chapter, non-completion rates drop considerably over time within each cohort, and do not settle at a certain level until well into the thirties.

Any analysis of young people's careers needs to handle this heterogeneity in sensible ways. Indeed, one major purpose of the present report is to deepen our understanding of the different trajectories followed by youth up to age 20 , to compare the patterns of trajectories across the four Nordic countries under study, and to link these patterns to labour market outcomes in adulthood. Simultaneously, many of the results and patterns reported in the subsequent chapters touch upon the overall picture mediated by the international and cross-Nordic comparisons presented in this chapter. In particular, several of the distinct characteristics of the Nordic countries, when compared to non-Nordic countries, will show up in the subsequent chapters. This holds true also for many of the pronounced differences between the Nordic countries pointed out above. In our analysis, we aim at positioning them in a broader, more dynamic context, with a view of improving our knowledge on the performance of young people in school and in the labour market. 


\section{General description of post- compulsory-school activities}

This chapter introduces the national datasets underlying the empirical evidence reported in this volume. It also provides a general description of the main post-compulsory-school activities in which the young people covered by our data have been engaged in, with the emphasis being on their situation up to age 20. In other words, in this chapter we give an overall picture of young people's activities over a time period which is often argued to be the most critical in terms of their future labour market outcomes.

Additionally, we introduce our preferred definition of young people lacking an upper secondary certificate. More precisely, we prefer to call these young people "non-completers", as compared to "completers", implying that they only have their compulsory-school exam still five years after having left primary education. As shown in the previous chapter, both Eurostat and OECD label such young people in a different way - early school leavers and dropouts, respectively - and also define them slightly differently. A major reason for using the term "noncompleters" is that, as will be highlighted below, large shares of them spend, in effect, a considerable amount of time as full-time students but without completing an upper secondary degree. Calling them early school leavers or school dropouts would, therefore, mediate a misleading picture of their actual situation. This, in turn, gives us also reason to contrast our non-completers' results to the early school leaving and dropout statistics reported by Eurostat and OECD for the Nordic countries, information that was briefly reviewed in Chapter 1 .

\subsection{The national datasets used}

This sub-chapter provides a general outline of the national datasets used in our subsequent analyses of young people in four Nordic countries. More details on our data are given in Appendix 1. 


\subsubsection{Longitudinal data for three cohorts}

The national datasets underlying our results are longitudinal in nature and compiled from large administrative registers maintained by the statistical bureau in the respective country. In particular, each national dataset covers three full cohorts of 16-year-olds: those who turned 16 in 1993, in 1998 and in 2003, respectively. The reason for choosing age 16 as our point of departure is that it represents the typical compulsoryschool-leaving age in the Nordic countries. Since we are able to cover all young people in these three cohorts, we need not worry about potential selection bias problems due to sampling. Apart from allowing overall comparisons across cohorts, these three youth cohorts may also highlight the role of the surrounding economy, as they have faced highly different business cycles when leaving compulsory school. For instance, those having left compulsory school in 1993, which represents a deep economic recession year in all four Nordic countries under study, could be expected to have encountered much higher employment barriers as compared to those having left compulsory school in 1998, representing a strong economic upturn year.

These three cohorts of 16-year-olds are followed on an annual basis up to the year 2008. Hence, all our results refer to pre-crisis years. In practice, the following-up period is thus of different length for the three cohorts. While the 1993 cohort of 16-year-olds can be followed for a total of fifteen years, up to age 31, the 1998 cohort of 16-year-olds can be traced for ten years, up to age 26 , but the 2003 cohort of 16-year-olds for only five years, up to age 21 . This means that we have information on all three cohorts up to age 21, on two cohorts (1993 and 1998) for ages 22 to 26 and on one cohort (1993) for ages 27 to 31 . This fact should be kept in mind in those situations where we merge the information on all three cohorts and present results also for age points beyond age 21 .

\subsubsection{Five main activities}

When following these 16-year-olds on their way in post-compulsory education and to the labour market, we distinguish between five main activities: full-time student, employed, unemployed, disability beneficiary ("pensioner") and "other". Allocating our young people across these five activities is not straightforward, though, as the five activities are not necessarily mutually exclusive. A young person may well switch, even repeatedly, between activities during a year. In this respect, we rely on their status at the time of observation in the data; say, the young person's main activity as registered for the last week of the year. However, 
even then the young person might be engaged in several activities at the same time, e.g. full-time studying and part-time employment.

We have tried to address the challenges related to the allocation of our young people across the five activity categories in the following way. As our point of departure we have chosen the labour-market-status indicator readily available in the Finnish administrative registers. This indicator is constructed by Statistics Finland in line with the recommendations of the International Labour Organization (ILO). In brief, this means that the young person needs to have an employment contract in force to be coded as employed; to be registered at public employment services (PES) for being coded as unemployed; to be registered as a disability beneficiary for being coded as a "pensioner"; and to be enrolled in education for being coded as a student. All young people not appearing in any of these large administrative registers fall into the dumping category of "other"; i.e., their activity is unknown. Since the official data sources used for the other three countries lack a similar indicator, the young people covered by the Danish, Norwegian and Swedish datasets have been allocated across the five activities in a way that follows as closely as possible the coding rules for the Finnish labour-market-status indicator.

The ILO hierarchy of these five activities is: employed, unemployed, pensioner, student, other. Hence, also full-time students are coded as employed, if they happen to have an employment contract in force at the time of observation. Since this way of coding full-time students was felt as highly unsatisfactory for our purposes (cf. the discussion in Chapter 1), all young people studying on a full-time basis were recoded as students in case they appeared in some other activity in the original data. This recoding was based on administrative school register data. Hence, all young people enrolled in an educational institution at the time of observation are in our datasets allocated into the activity labelled "studying" or "student". A comparison of school-to-work-transition patterns over the ages 16 to 20 based on recoded and non-recoded student information provides strong support for our recoding exercise in terms of more realistic early trajectories of young people (see e.g. Asplund and Vanhala, 2013, for Finland, and Barth and von Simson, 2012, for Norway). 


\section{Box 2.1 Main activities}

The following main activities are identified based on our national datasets: studying, employed, unemployed, disability beneficiary and a residual group labelled "other", which mainly consists of young people not found in any of the broad administrative registers from which our national datasets are compiled. If a young person appears in several registers at the same time, the following priority is given: activities in the labour force (employed/unemployed) override activities outside the labour force (disability beneficiary and "other"). There is one important exception to this rule, though: those young people registered as fulltime students are always treated in our datasets as students, even when they are in the employment or the unemployment register while studying.

A careful allocation of young people across a reasonable number of relevant activities can be expected to produce important information on post-compulsory-school trajectories, as well as on crucial changes over time (across cohorts) in early school-to-work transition patterns. However, the changes in activities possibly observed over time are not necessarily merely due to changing behaviours or preferences of young people. Apart from changes in the surrounding economy, these shifts in activity patterns may also be driven by reforms of the institutional setting. For instance, a decline in the unemployed share of young people of a particular age may be explained by higher preferences for work and/or improved employment opportunities, but may also reflect tightened conditions for young people of this age when it comes to their possibilities to register as unemployed and/or their eligibility for receiving unemployment benefits. Likewise, a decline in the share of young people of a certain age on disability benefits is not necessarily the result of young people having become healthier but more likely the outcome of fundamental changes in the disability benefit system. Whenever relevant in the subsequent analyses, we will bring forward major reforms in the institutional settings of the four Nordic countries under study that are likely to have contributed to distinct changes observed in young people's early school-to-work trajectories and labour market outcomes. 


\subsection{Main activities of young people - the overall pattern}

We start our general description of young people's post-compulsoryschool activities by showing the overall distribution of our three country-specific youth cohorts across the five main activity categories defined above: full-time studying, employment, unemployment, disability benefits ("pensioner") and "other" (outside education, labour force and disability arrangements). This is done separately for each age point between 16 and 20/21 and then for two more age points slightly later in life, at age 26 and age 31 . While each country figure is based on pooled information for all three youth cohorts, the table accompanying each figure displays the corresponding activity shares separately by gender and by cohort at one particular point in time, viz. when our young people turned 21.

\subsubsection{Denmark}

The first figure and its complementing table (Figure 2.1a and Table 2.1a) highlight the situation for Denmark. The figure reveals that the share of compulsory-school-leaving youth continuing directly in education is extremely high (about 90\%) among 16-year-old Danes, but declines thereafter: at a rather slow pace up to age 18 but much more rapidly over the next two years, up to age 20. By age 21 , the share of full-time students has shrunk to about $44 \%$. In parallel, the share of young people in employment has increased to approximately the same level. However, these average shares based on pooled cohort data conceal substantial variation across cohorts, as shown in Table 2.1a. In particular, the share of 21-year-olds engaged in full-time studies has grown remarkably, from close to $40 \%$ for the 1993 cohort to almost $48 \%$ for the 2003 cohort. Simultaneously, the share of 21-year-olds in employment reveals a declining trend across cohorts. 
Figure 2.1a: Denmark: Average distribution (\%-share) of young people across main activities up to age 31, based on pooled information on all three youth cohorts

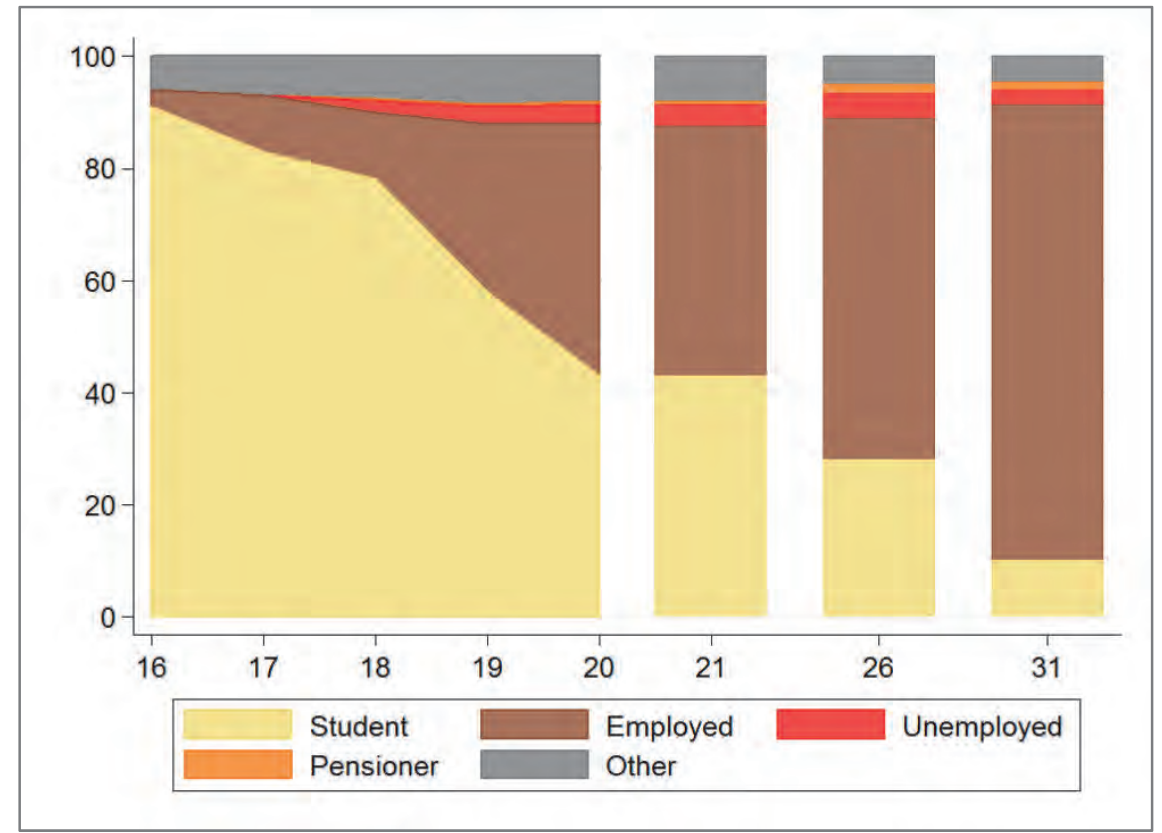

Note: For definitions of the five main activities, see sub-chapter 2.1.

Table 2.1a: Denmark: Average distribution (\%-share) of young people across main activities at age $\mathbf{2 1}$, based on pooled information on all three youth cohorts (all and by gender) and separately by cohort

\begin{tabular}{|c|c|c|c|c|c|c|}
\hline \multirow[t]{2}{*}{ Activity } & \multicolumn{3}{|c|}{ All three cohorts pooled } & \multicolumn{3}{|c|}{ By cohort of 16 -year-olds } \\
\hline & All & Males & Females & 1993 & 1998 & 2003 \\
\hline Full-time student & 43.7 & 40.6 & 47.1 & 39.5 & 44.2 & 47.5 \\
\hline Employed & 43.2 & 46.5 & 39.7 & 47.5 & 41.5 & 40.5 \\
\hline Unemployed & 3.9 & 4.0 & 3.8 & 4.8 & 5.6 & 1.4 \\
\hline Pensioner (disability benefits) & 0.7 & 0.8 & 0.6 & 0.5 & 0.7 & 0.8 \\
\hline Other (inactivity) & 8.5 & 8.2 & 8.9 & 7.7 & 8.1 & 9.8 \\
\hline Total & 100.0 & 100.0 & 100.0 & 100.0 & 100.0 & 100.0 \\
\hline$\%$-share in the pooled dataset & 100.0 & 51.7 & 48.3 & 34.4 & 31.0 & 34.6 \\
\hline
\end{tabular}

Note: For definitions of the five main activities, see sub-chapter 2.1 .

The smooth transition of Danish youth from post-compulsory education into working life displayed in Figure 2.1a is a distinct feature of the country's upper secondary education system: almost all students in initial vocational education and training (VET) are in some form of combined school- and work-based learning (see Box 2.2). Simultaneously there is by now strong evidence showing that graduates from such programs have better employment prospects than graduates from more 
"school-based" upper secondary education systems, especially in times of economic downturns (e.g. Cedefop, 2012).

The share of the unemployed starts to grow after age 17 but remains low: less than $4 \%$ still at age 21 . Again we see considerable variation across cohorts, though: the unemployment share among 21-year-olds increased to $5.6 \%$ in the 1998 cohort, but dropped to $1.4 \%$ in the 2003 cohort. The unemployment share in the two older cohorts is well in line with the LFS shares shown in Figure 1.5a of Chapter 1, while it is not entirely clear why the corresponding share for the 2003 cohort is lower than for the other two cohorts.

The share of young people outside both education and the labour force is about $7 \%$ among the 16 -year-olds, and increases only slightly up to age 21 (some 9\%), with those on disability arrangements contributing only marginally to this share. However, also for these groups of young people we can observe clear-cut trends over cohorts: both the share of young people on disability benefits and the share of those belonging to the dumping category of "other" show a steady upward trend across cohorts.

The distribution of 21-year-old men and women across the five activity categories points to a strong preference among young women to continue in full-time education, and among young men to start working (Table 2.1a). A main reason for this gender difference is that the VET system attracts more young men than women, and that many students delay their entry into the VET system until they are in their twenties. When it comes to NEET activities (unemployment, disability arrangements and other inactivity) there are no conspicuous differences between the male and female distributions.

The share of young people in employment continues to grow rapidly when moving beyond the age of 21 (Figure 2.1a). By age 26 it has increased to $60 \%$ and by age 31 to $81 \%$, mainly at the expense of enrolment in full-time studies: the share of students is down at $10 \%$ among the 31-year-olds. The share of the unemployed is conspicuously low at age 31, also when compared to the situation at younger ages. The share of young people on disability benefits increases slightly after age 21 while the share of young people in other inactivity is declining. These opposite trends seem, however, to be mainly explained by a shift from other inactivity into disability; taken together, the share of young people outside both education and the labour force is approximately the same at age 26 and age 31 (about 7\%). 


\section{Box 2.2 Denmark - a brief outline of the relevant institutional framework}

\section{Upper secondary school system}

Education in Denmark is compulsory from the age of 6 and lasts for 9 years up to the 9 th grade. The primary school system comprises both a voluntary 10th grade and a voluntary pre-school year (beginning at the age of 5). The Danish municipalities administer the primary school system, which is regulated by the Ministry of Education.

After completing compulsory school young persons can apply for upper secondary education. There are two main tracks in the Danish secondary education system: an academic track and a vocational track. The main academic track lasts three years and takes place in the "Gymnasium". Most applicants are admitted but the school can reject an applicant, if it is unlikely that the young person is able to pass the exams. An exam from the Gymnasium is normally a prerequisite for admission to universities. The vocational educational track (VET) begins normally with a basic course of a duration between 20 to 25 weeks in either a technical college or a commercial college. After the basic course follows the main course, which is a "dual" educational program: the main part of the training takes place in companies, but during some time periods the participants attend courses in colleges. The main course typically takes $3-31 / 2$ years, but can be shorter or longer depending on the program (from $1 \frac{1}{2}$ up to 5 years). There are about 110 different VET programs.

There is free admission to the basic VET program, but participation in the main course at a company demands that the participant finds employment at a company. Employment and training in companies is regulated by apprenticeship contracts between the company and the apprentice. The share in a youth cohort that obtained a training contract with an employer was about $45 \%$ during the years under scrutiny in this report (Albæk, 2009). This share is much larger than in most European countries with the exception of Austria, Germany and Switzerland.

A particular feature of the Danish VET system is the high age of the apprentices. According to Figure 1.8 of Chapter 1, the average age of graduation from the Danish VET system is 28 , whereas students in general programs typically graduate when they are 19 years old. The age of apprentices has increased over a long time period (Albaek, 2005). Lack of training slots in companies is a much debated policy issue and various employment subsidies have been in effect (Albæk, 2012). 
Measures directed towards young people outside education and the labour market

Unemployment benefits: A prerequisite to receive unemployment benefits in Denmark is membership of an unemployment insurance fund. These insurance funds are private organisations in principle, but the different provisions regulating their activities are regulated by the state and the state also provides most of the funding of unemployment benefits. A condition for receiving unemployment benefits was (until 2010) employment for half a year during the last three years. Also eligible for unemployment benefits are persons who have finished higher education or an education in the VET system (of at least 18 months). The maximum amount of benefits is $90 \%$ of the previous wage but there is also a general maximum, which most unemployed workers receive, except for workers who received low wages before the unemployment spell.

Social assistance: When young people turn 18, they are entitled to social assistance, if they have no other means of income (parents are supposed to support their children economically until the age of 18). No other means include that they have not been able to find a job, that they have no savings and that they do not have a spouse, who can support them. The amount of social assistance is means tested, which for example implies that young persons with children receive higher social assistance than young people without children. Social assistance is administered by the municipalities. If the municipality assesses that a young person is able to work, then the person has to register as an unemployed jobseeker. This implies, in turn, that the person is supposed to take a job offered through the labour market authorities.

Active labour market policy: There are various programs for young people who do not work and who are not enrolled in education. The programs include both training programs and programs that aim at giving young people work experience. Enrolment in these programs goes under the name "activation". Over the years, several reforms have been enacted to induce young people either to participate in education or take a job. A major reform took place in 1994, according to which young people under 25 on social assistance have the "right and duty" to participate in education of at least 18 months of duration. A reform in 2003 extended this "right and duty" to young people under 25 on unemployment benefits provided they had been unemployed for more than six months. According to another reform, which also took place in 2003, young people under 25 can, at a maximum, receive social assistance corresponding to the amount that young people participating in education receive (the Danish system of income support to persons participating in education - Statens uddannelsesstøtte (SU) - is relatively generous). 
Disability benefits: To be eligible for disability pension, the person has to have a physical or psychical illness that reduces his/her work ability permanently. A disability pension can be granted from age 18 and stops at retirement age. The right to receive a disability pension is, as a general rule, dependent on Danish citizenship and residence in Denmark. Eligibility for a full pension requires that the pensioner has been a Danish resident at least $4 / 5$ of the years after the pensioner turned 15 to the time when the pension is granted.

There is only one disability pension scheme in Denmark, which is financed out of general public revenue. No requirement of previous work experience has to be fulfilled in order to obtain a disability pension and neither is the level of the pension related to previous work income which is, in effect, also the case for public old-age pensions. This is in contrast to many other countries, where some amount of work experience is a prerequisite for receiving a disability pension and where the level of the pension is related to previous earnings. Disability pensions are granted by the municipalities, which also differs from practices in most other countries. Most cases start with an application from persons who want to obtain a disability pension, but municipalities can also initiate cases for persons who reside in the municipality. The decision of the municipality can be appealed to an appeal agency (ankestyrelsen). A substantial revision of the disability pension system was enacted in 2013. A main provision is that persons below the age of 40 , as the point of departure, cannot obtain disability pensions. In line with the 2003 reform of the Swedish disability system for young people, the Danish reform aims at improving the rehabilitation of young people with disabilities and at providing them, in the first place, with a so-called resource plan (ressourceforløb) for a limited time period (up to five years).

Prior to 2003, disability pensioners were classified in categories according to their residual work ability and the level of the pension was dependent on categorization (highest, middle, enhanced ordinary and ordinary disability pension). Starting in 2003 the categorization was abandoned for new entrants to the disability pension system and only one type of disability pension is granted. Persons on the old scheme can apply to enter the new scheme.

A special trait of the Danish system is the so-called flexible-job scheme, which is also targeted to persons with permanently reduced work capacity. The scheme consists of a wage subsidy that amounts to either $1 / 2$ or $2 / 3$ of the salary. The wage subsidy is paid to employers that hire the persons participating in the flexible-job scheme. Persons in this scheme are not included as pensioners in our analysis. 


\subsubsection{Finland}

Next we turn to the corresponding information for Finland (Figure 2.1b and Table 2.1b). A large majority of the 16-year-olds continue in education either directly after completing compulsory school or one year later, at age 17 , as clearly indicated in the figure by the increase in the share of full-time students among the 17-year-olds. Concomitantly, there is a sharp decline in the share of compulsory-school leavers outside both education and the labour market ("other") when youngsters re-enter school after having spent a year in non-education and non-employment activities. Then follows rapidly growing numbers of 18- and 19-yearolds moving from upper secondary education into working life, though occasionally also into unemployment or entirely outside the labour force. This "two-step" transition into working life reflects, at least in part, a reform of initial vocational education turning by 2001 all 2-year vocational tracks into 3-year tracks (see Box 2.3). Since Figure 2.1b is based on pooled information on the three youth cohorts under scrutiny, the share of young people enrolled in full-time education is, by necessity, a mix of students having followed 2-year and 3-year vocational tracks in upper secondary education.

After age 19, the share of full-time students starts to increase, mainly at the expense of working, and by age 21 approximately half (49\%) of all these young Finns were studying on a full-time basis. Hence, the early post-compulsory-school transition pattern observed for Finland is characterised by two conspicuous "pauses": a delayed start in upper secondary education and a delayed start in tertiary-level education. We will return to these delayed starts in our subsequent analyses. Finally it is worth noting that the variation across cohorts in the share of full-time students at age 21 is minor ( $\pm 2-3 \%$, Table $2.1 \mathrm{~b}$ ). Hence, compared to the situation in Sweden and especially in Denmark, the share of young Finns in full-time studies has remained remarkably stable across cohorts. 
Figure 2.1b: Finland: Average distribution (\%-share) of young people across main activities up to age 31, based on pooled information on all three youth cohorts

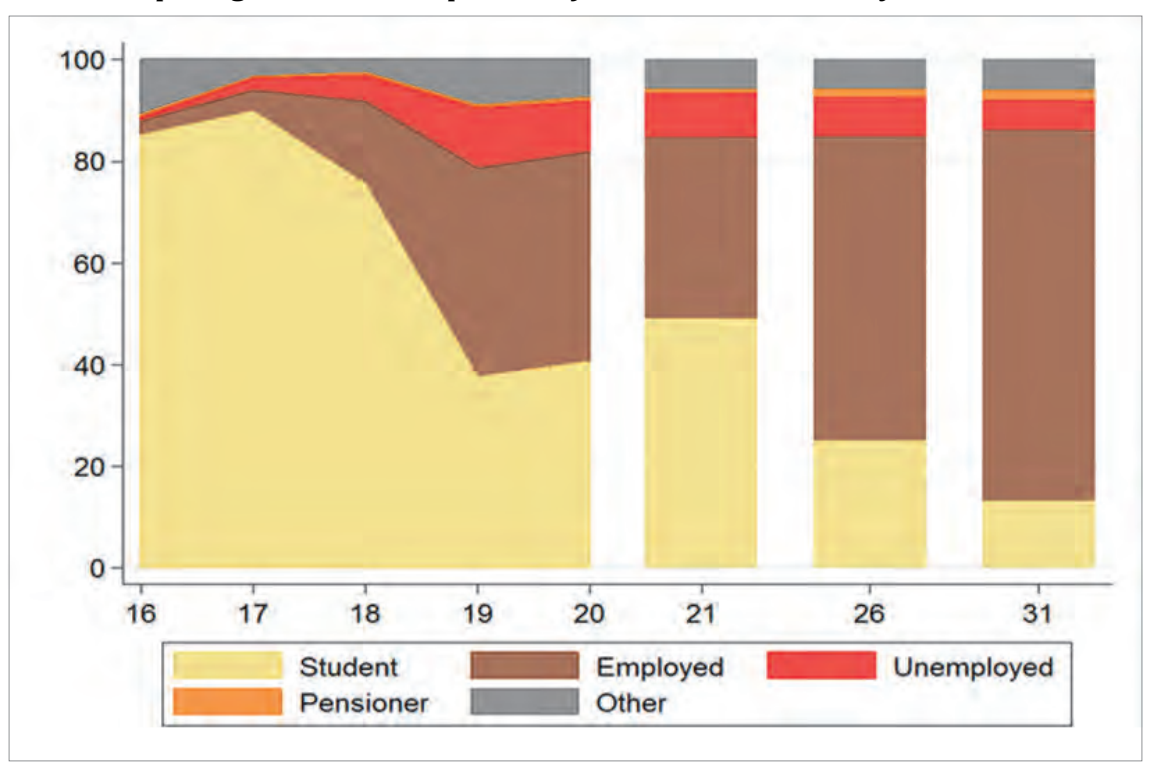

Note: For definitions of the five main activities, see sub-chapter 2.1

Table 2.1b: Finland: Average distribution (\%-share) of young people across main activities at age 21, based on pooled information on all three youth cohorts (all and by gender) and separately by cohort

\begin{tabular}{|c|c|c|c|c|c|c|}
\hline \multirow[t]{2}{*}{ Activity } & \multicolumn{3}{|c|}{ All three cohorts pooled } & \multicolumn{3}{|c|}{ By cohort of 16 -year-olds } \\
\hline & All & Males & Females & 1993 & 1998 & 2003 \\
\hline Full-time student & 49.0 & 42.0 & 56.5 & 48.0 & 50.6 & 48.4 \\
\hline Employed & 35.6 & 42.0 & 28.9 & 34.6 & 34.0 & 38.6 \\
\hline Unemployed & 8.6 & 10.1 & 7.1 & 10.9 & 8.4 & 6.5 \\
\hline Pensioner (disability benefits) & 1.0 & 1.1 & 0.8 & 0.9 & 1.0 & 1.0 \\
\hline Other (inactivity) & 5.7 & 4.8 & 6.7 & 5.7 & 6.0 & 5.4 \\
\hline Total & 100.0 & 100.0 & 100.0 & 100.0 & 100.0 & 100.0 \\
\hline$\%$-share in the pooled dataset & 100.0 & 51.3 & 48.7 & 33.9 & 34.6 & 31.5 \\
\hline
\end{tabular}

Note: For definitions of the five main activities, see sub-chapter 2.1 .

A comparison across cohorts of labour market activities at age 21 points to improved employment, as well as unemployment (Table 2.1b). However, the much more favourable situation in this respect for the youngest (2003) cohort is not necessarily merely due to truly better employment opportunities. Obviously it also reflects intensified active labour market policies directed towards unemployed young people (cf. Box 2.3). This aspect is not explored further in this context, though, as we throughout overlook the quality of the employment relationship, including the fact that the employment contract may conceal a (temporary) subsidised job. The share of 21-year-olds engaged in unknown activities ("other") outside 
both education and the labour market has remained strikingly unchanged over the three cohorts, as has also the share of disability beneficiaries.

A comparison across genders at age 21 reveals that the most conspicuous discrepancies show up in the shares of full-time students and the employed: young women continue disproportionally in education compared to young men, whereas young men are much more likely to move into working life, a situation also observed for the other Nordic countries. Nonetheless, the risk of unemployment seems to be approximately the same, one in five, for both genders. A slightly larger share of young men was on disability benefits at age 21 with, in contrast, the share of those in other inactivity being notably larger among young women of this particular age.

By age 26, the share of full-time students had declined to $25 \%$, and by age 31 only about $13 \%$ were enrolled as full-time students. Concomitantly, the share in employment increases rapidly, to $60 \%$ among the 26 year-olds and $73 \%$ among the 31-year-olds. The share in unemployment declines slightly, whereas the share on disability benefits shows a weakly growing trend, standing at $2 \%$ among the 31-year-olds. The share of young people in the dumping category of "other" (outside education, the labour market and disability arrangements) seems to remain approximately unchanged from age 21 onwards, that is, close to $6 \%$.

\section{Box 2.3 Finland - a brief outline of the relevant institutional framework}

\section{Upper secondary school system}

All children permanently resident in Finland must attend compulsory education, which starts in the year of the child's seventh birthday and finishes when the syllabus of the 9-year comprehensive school education is completed. After having received a leaving certificate from comprehensive school education in the same or in the previous year, a student may continue in additional voluntary basic education (10th grade).

The post-compulsory upper secondary level comprises general education and initial vocational education and training (VET). The entire age cohort is in principle, and increasingly also in practice, granted a study place in upper secondary education. The general upper secondary school is based on courses with no specified year-classes. The scope of the syllabus is three years but the studies may be accomplished in two, three or four years. This "class-free" system was introduced in the period 1994 to 1996. General upper secondary education is primarily free of charge for students. The upper secondary school ends in a matriculation examination which gives general eligibility to further education in universities or polytechnics. 
The initial VET qualification takes three years of full-time study, unless prior learning can be counted towards the qualification. Up to 2001, it was also possible to complete an initial VET education in two years. A vocational qualification gives general eligibility for polytechnic and university studies. There are no tuition fees in initial VET. Training for initial vocational qualifications is available mainly in vocational institutions, but also in the form of apprenticeship training. Vocational institutions-based education comprises about five months of on-the-job learning, whereas most of the apprenticeship trainings takes place in the workplace, often supplemented with only short theoretical education at a vocational institution. The apprenticeship training track is annually chosen by some $9 \%$ of vocational students. Since 1994 it has also been possible to take a competence-based qualification. This system is intended to enable working-age adults to gain qualifications without necessarily attending formal training. Less than $20 \%$ of the annually awarded vocational qualifications are competence-based qualifications.

Students in upper secondary schools have, since 1998, had the possibility to combine general and initial vocational education and complete a so-called double exam. These types of studies typically take three to four years to complete.

\section{Measures directed towards young people not in education or employment}

Unemployment benefits: Unemployment insurance covers three distinct types of benefits: earnings-based unemployment insurance, basic unemployment allowance, and labour market support. Only the labour market support requires no employment history. Accordingly, a notable share of these beneficiaries comprises young people. This flat-rate means-tested benefit was introduced in 1994 and is paid for an unlimited period. The eligibility conditions for receiving labour market support were tightened in 1995 and 1996 for the unemployed under 25 years-of-age lacking a vocational qualification.

Active labour market policy (ALMP) measures and programs: The deep recession in the early 1990s caused an extremely dramatic increase in unemployment. Moreover, unemployment decreased only slowly after the start of economic recovery in 1994, resulting in high and persistent structural unemployment. This situation initiated both PES reforms and measures in ALMPs. In particular, Finnish labour market policy was thoroughly reformed in 1998, followed by further actions for this reform completed in 2001 (Räisänen et al., 2012). A second wave of PES reforms was undertaken in 2004-2006. The main types of ALMPs are employment subsidies and labour market training. Also practical training programs are quite extensive in volume. The deep economic recession 
in the early 1990s initiated, inter alia, more use of preparatory labour market training, introduction of job-search training in labour market training programs, explicit targeting of employment programs at the corporate sector in particular, and cuts in public-sector employment programs. Especially since 2005, the reforms in ALMPs have been designed to provide and support education.

Young people are an integrated part of this labour market policy framework, with the relevant ALMPs depending on their age and employment history. Special attention has thereby been paid to the "youth society guarantee" introduced in 2005. It covered young unemployed persons under the age of 25 and aimed to enhance the placement of these young people in education and the labour market, to prevent prolonged unemployment, and to support sustainable career solutions. Job-search plans were made a key instrument. At the beginning of 2013 , this system was replaced by a "youth guarantee" offering a study place or a job, training or rehabilitation, or other relevant activity after three months of unemployment to those aged 15 to 24 , as well as those under 30 who have recently obtained a vocational or academic qualification. Both guarantee systems have been subject to evaluations (see e.g. Asplund and Koistinen, 2014).

Social assistance: Social assistance is a last resort form of income security intended to secure at least the necessary income referred to in the Constitution of Finland, as well as to promote the independent coping of individuals and families. Local authorities pay means-tested social assistance when the income and resources of a household are insufficient to cover necessary daily expenses. Since the beginning of 2006, social assistance is divided into three categories: basic, supplementary and preventive. The aim of preventive social assistance is to help enable individuals and families to manage independently and to prevent social exclusion and long-term dependence on social assistance.

Disability benefits: The young people recorded to be disability beneficiaries in the Finnish dataset receive a disability pension according to the registers compiled jointly by the Finnish Centre for Pensions (ETK) and the Social Insurance Institution of Finland (Kela). A disability pension may be granted either in the national pension scheme or in the statutory earnings-related pension scheme. The liability to take out insurance under the earnings-related pension legislation starts at the beginning of the month following the person's 18th birthday. The national pension scheme, in turn, covers all persons aged 16 to 64 who have permanently resided in Finland for at least three years after turning 16. Exceptions from this required period of residence are awarded to: (1) those having become incapable of working before the age of 19 while resident in Finland and (2) those receiving a disability allowance for persons under the age of 16 when turning 16 . In contrast to the earnings-related pension, the national 
funded by the state and paid at a flat rate with income testing. Irrespective of the scheme under which the disability pension is awarded, the following applies. The illness needs to significantly reduce the person's work ability. The pension is awarded until further notice or for a specific time period. The time-limited benefit (since 1996 called a cash rehabilitation benefit) is granted if the treatment or rehabilitation can be expected to improve, at least in part, the person's working capacity. Accordingly a cash rehabilitation benefit is always to be accompanied by a treatment or rehabilitation plan.

These parallel pension systems have been subject to a number of reforms over the past decades. One major change was made in 2005 , resulting in a further differentiation of the two systems. Of particular relevance in this context is a reform related to the medical requirements for disability pension eligibility under the national pension scheme. In particular, from August 1, 1999 onwards, a national disability pension was no longer granted to persons under the age of 18 until their prospects for vocational rehabilitation had been clarified. By April 1, 2002, this age limit was increased to 20 years thus covering all persons aged 16 to 19 , although there are a number of the exceptions to this rule. In the earnings-related pension scheme, it is required that the incapacity for work can be estimated to last for at least one year. A noteworthy change in this scheme is, however, that the minimum requirement for previous earnings was markedly reduced in 2005, which resulted in a notable increase in the earnings-related pension beneficiary caseload.

\subsubsection{Norway}

The situation for Norway is highlighted in Figure 2.1c and Table 2.1c. The share of full-time students evolves up to age 20 in a way that is strikingly similar to the trend observed for Finland (cf. Figure 2.1b above). But the reason behind the decline in the share of full-time students at age 18, as compared to the situation at age 17, is different for Norway: most probably this pattern is due to large shares of vocational students dropping out between their 2nd and 3rd year due to a lack of apprenticeship places; a contention that receives further support when comparing the shares in education at age 17 and 18 for those having completed and those not having completed an upper secondary degree by age 21 (no dropout is observed for the completers).

Compared to the situation in Finland, however, the share of compulsory-school-leavers continuing in full-time education starts out from a clearly higher level, and this share also declines less sharply with age. By 
age 21, every second Norwegian is enrolled in full-time education. This share is similar to that observed for Finland and clearly higher than that observed for Denmark and Sweden.

The share of young Norwegians in employment increases notably after age 18, and by age 21 about one-third of them are working. Young people thus seem to start moving into working life somewhat later in Norway than in Denmark and Finland, and their employment share also tends to remain at a lower level especially when compared to Denmark and Sweden. Norway is rather characterised by an increasing share of young people moving outside both education and the labour force ("other") after leaving compulsory school. This share is as high as $16.5 \%$ among the 19 -year-olds, but shrinks to about $9 \%$ by age 21 . However, this is still a share that is higher than for Denmark and much higher than for Finland and Sweden. In contrast, the share of young Norwegians on disability benefits remains low: it is slightly lower than in Sweden but marginally higher than in Denmark and Finland. Also the share of the unemployed shows only a weakly growing trend up to age 21 where it lands at 5.4\%. This share is clearly lower than for Finland and Sweden, but higher than for Denmark.

Figure 2.1c: Norway: Average distribution (\%-share) of young people across main activities up to age 31, based on pooled information on all three youth cohorts

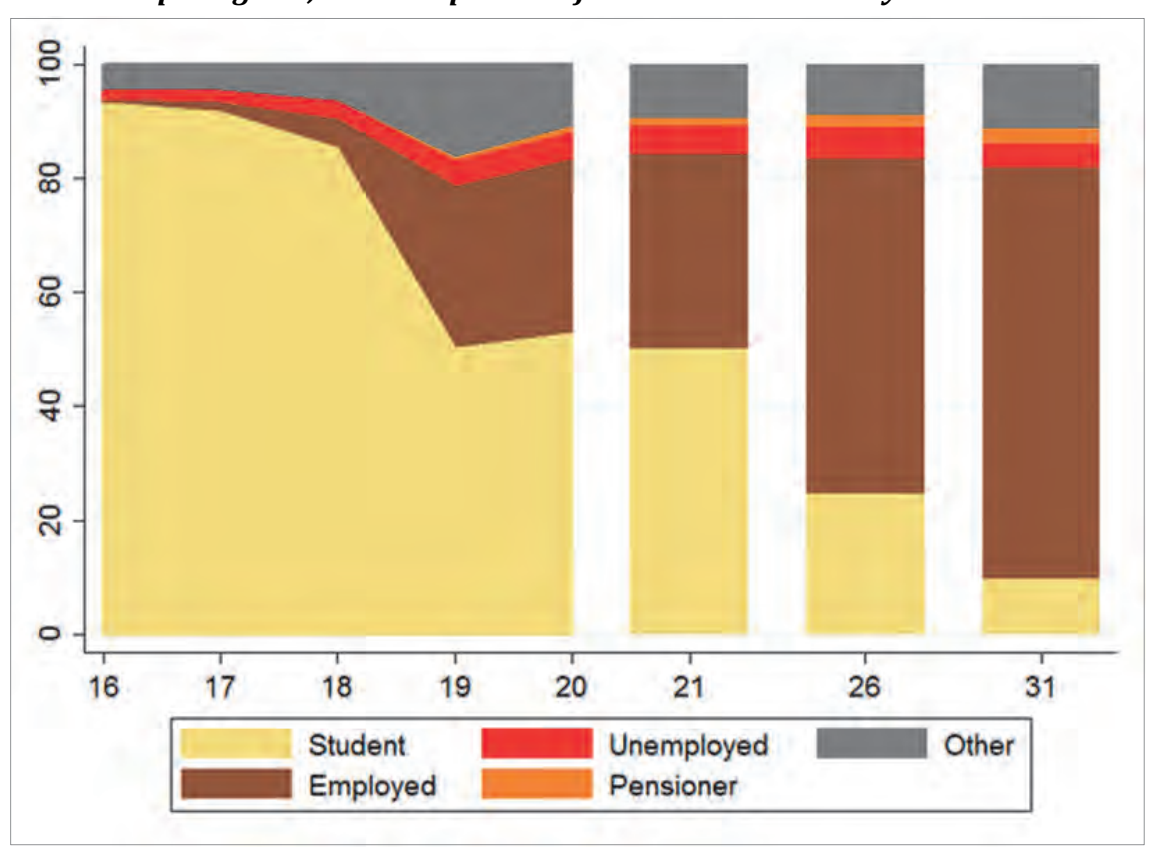

Note: For definitions of the five main activities, see sub-chapter 2.1. 
Table 2.1c: Norway: Average distribution (\%-share) of young people across main activities at age 21, based on pooled information on all three youth cohorts (all and by gender) and separately by cohort

\begin{tabular}{|c|c|c|c|c|c|c|}
\hline \multirow[t]{2}{*}{ Activity } & \multicolumn{3}{|c|}{ All three cohorts pooled } & \multicolumn{3}{|c|}{ By cohort of 16 -year-olds } \\
\hline & All & Males & Females & 1993 & 1998 & 2003 \\
\hline Full-time student & 50.1 & 43.9 & 56.5 & 49.4 & 50.3 & 50.4 \\
\hline Employed & 34.0 & 38.7 & 29.1 & 33.8 & 34.3 & 34.0 \\
\hline Unemployed & 5.4 & 7.1 & 3.7 & 4.1 & 7.1 & 5.1 \\
\hline Pensioner (disability benefits) & 1.4 & 1.3 & 1.4 & 1.4 & 1.2 & 1.5 \\
\hline Other (inactivity) & 9.1 & 9.0 & 9.3 & 11.3 & 7.1 & 9.0 \\
\hline Total & 100.0 & 100.0 & 100.0 & 100.0 & 100.0 & 100.0 \\
\hline$\%$-share in the pooled dataset & 100.0 & 51.2 & 48.8 & 32.7 & 32.9 & 34.4 \\
\hline
\end{tabular}

Note: For definitions of the five main activities, see sub-chapter 2.1.

A conspicuous feature of Norway is the stability of the average distribution across main activities over the three cohorts under scrutiny (Table 2.1c). The shares in full-time education and in employment are more or less the same for all three cohorts. The changes across cohorts in the shares of young Norwegians showing up in NEET activities (unemployment, disability benefits and other inactivity) are quite small, revealing no clear-cut trend over time. Rather there seems to have occurred occasional trade-offs between unemployment, on the one hand, and disability benefits and other types of inactivity, on the other hand.

The average distribution across main activities of young men and young women turning 21 unravels no surprises when compared to the overall picture obtained for the other three countries. Also in Norway, young women have a stronger preference to continue in full-time education as compared to their male peers, many of whom prefer to move from school into working life. Additionally, a larger share of young men than of young women encounters unemployment at age 21 . The higher unemployment risk of young men is retained also when account is made for the gender difference in employment shares. However, when it comes to the two types of inactivity (disability benefits and "other"), the distributional shares are only marginally different for young men and young women.

The share of young people studying on a full-time basis declines dramatically after age 21 . By age 26 it is down to about $25 \%$ and by age 31 it has dropped below $10 \%$. At the same time, employment becomes the dominating activity with $72 \%$ of the 31 -year-olds working. The combined share of young people in NEET activities remains approximately unchanged, although there seems to occur continuous shifts between these three non-education and non-employment statuses, notably from unemployment into inactivity. 


\section{Box 2.4 Norway - a brief outline of the relevant institutional framework}

\section{Upper secondary school system}

Education in Norway is compulsory from the age of 6 until the age of 16. After completion of compulsory education, every young person has a statutory right to attend three years of upper secondary education free of charge. This right must be utilized during a period of five consecutive years (six for some vocational courses), and must be started within five years after completed compulsory school. Enrolment is almost universal: $96-97 \%$ of each youth cohort leaving compulsory school has a direct transition to upper secondary education. This share has barely changed since 1993 (Falch and Nyhus, 2009).

When applying for upper secondary education, the youth may choose between two main tracks: academic and vocational studies. Around half of the students opt for the academic track, which prepares for further studies in universities and colleges. The rest of the youth start a vocational study program, which gives qualifications for work in a number of different occupations. A standard course through an academic study program lasts three years, while most of the vocational programs follow a $2+2$ model: two years of school-based training followed by two years of apprenticeship in a company. There is, however, no guarantee of an apprenticeship-training-place and the supply of apprenticeships is shown to vary with the economic situation (Høst, 2008). Youth who do not receive an offer of an apprenticeship are entitled to a third year in school.

The statutory right of upper secondary education for young people as well as the integration of apprenticeships through the $2+2$ model were introduced in 1994, and are some of the main components of Reform 94 - a major educational reform that reorganized the entire upper secondary school system in Norway. The reform narrowed down the possible routes towards an upper secondary degree, and aimed at increasing the throughput of students. While the reform seems to have been somewhat successful, the non-completion rates are still high: around $30 \%$ of each youth cohort fails to complete their education with a formal certification within five years after enrolment. The non-completion rates have been remarkably stable since the introduction of Reform 94, and are almost twice as high for vocational students as for academic students. 
Measures directed towards young people outside education and the labour market

The youth guarantee and active labour market programs: Young people aged 16 to 21 who either do not apply for or interrupt their upper secondary education are under the responsibility of the Follow-up service. This service was established in 1994 in an effort to meet the challenges related to high dropout rates, and acts as a safety net. Through individual guidance, advice and practical assistance youth are directed into activities which aim at improving their access to the labour market. In addition to the Follow-up service, youth who have recently left the education system and have not yet found a job are assured participation in an active labour market program through the Youth Guarantee. The term guarantee may be misleading in this context, as the arrangement is not legally binding but rather reflects a clearly stated policy ambition. Initially, the guarantee concerned 16-19 year-old youth, but with the intensified efforts in the period 1995 to 1998 to provide active labour market programs the guarantee was temporarily extended to also cover 20-24 year-old long-term unemployed. This extended youth guarantee has been evaluated by Hardoy et al. (2006) who find that the guarantee increased the participation of 20-24 year-olds in programs and also improved their transition from unemployment into employment. The extended youth guarantee was reintroduced in 2009.

Unemployment benefits: The unemployment insurance scheme in Norway is based on previous labour market income. In order to receive unemployment benefits, one must register as a jobseeker at the local employment office and actually apply for work. In addition, one must have been paid wages of at least 1.5 times the National Insurance Scheme basic amount (BA) in the last calendar year, or at least 3 times the BA over the past three full calendar years. The replacement rate was $62.4 \%$ in 2012, and the benefit covers up to 6 times the BA.

Young school-leavers do not in general qualify for unemployment benefits, as they do not fulfil the income requirements. The incentives to register as unemployed for youth are thus low. The majority of non-/unemployed youth may instead be classified as NEETs (Not in Employment, Education or Training). 
Social assistance: Youth who are not able to financially support themselves through employment or other means may receive social assistance. The benefit is temporary, and the aim is to secure financial independence. In order to be entitled to social assistance, all other options must have been considered. This includes gainful employment, own savings and other financial rights, such as unemployment benefits. The amount received is means-based, and each case is specifically and individually assessed. It is possible to receive social assistance as a supplement to other income, if this income is too low to cover basic subsistence costs. Often there are certain conditions attached to the entitlement of social assistance, but these conditions are always set on an individual basis. For instance, non-employed individuals may have to register as jobseekers or participate in an active labour market program in order to receive the benefit.

Disability benefits: Youth who have reduced work capacity of at least $50 \%$ due to physical or mental health problems may be eligible for disability benefits. The general rule is that after 12 months on sick leave, one can apply for either temporary or permanent disability benefits, with a replacement rate of approximately $66 \%$. Young persons with no previous work experience may receive a minimum amount according to the guidelines established for the old-age pension. In addition, the individual has to be at least 18 years old (16 years old until 1998 ) in order to apply. In the period analysed in this report (1993 to 2008), the temporary disability benefits consisted of medical and vocational rehabilitation benefits as well as a time-limited disability pension (introduced in 2004). In 2010, the three benefits (medical and vocational rehabilitation, and time-limited disability pension) were combined into one: The Work Assessment Allowance. This allowance has a maximum duration of four years.

The rehabilitation benefits may be received for a maximum period of 52 consecutive weeks, while the time-limited disability benefit has an upper time limit of four years. If the health problems persist after this period, the person may apply for a permanent disability pension. The permanent disability may be either full or partial, depending on the remaining work capacity of the person. Normally, a permanent disability pension lasts until it is replaced by an old-age pension at the age of 67 , and there is no re-testing of the individual's work capacity. 


\subsubsection{Sweden}

Finally we turn our focus to Sweden. As illustrated in Figure 2.1d, the cross-activity distribution up to age 21 of the three Swedish youth cohorts under scrutiny can basically be described as an extreme version of the overall patterns displayed for Finland and Norway. More precisely, also Swedish 16-year-olds typically continue directly in postcompulsory education, occasionally after a year spent outside both education and the labour market: the share of continuing 16-year-olds is almost $94 \%$ and increases to over 95\% among the 17 -year-olds. Most young Swedes stay in upper secondary education for three years (see Box 2.5), after which follows a remarkably strong shift into working life: the share of full-time students drops from about $93 \%$ at age 18 to $37 \%$ at age 19 , whereas the share of employed grows from below $3 \%$ to $42 \%$. Hence, the magnitude of the school-to-work transition occurring at this age is even stronger than in Finland, resulting in a situation with only about $40 \%$ of 19 -year-old Swedes being enrolled in full-time education, a share similar to that observed for Finland (despite the two countries' quite different situations among 16-to-18-year-olds). The corresponding share is notably higher in Denmark and Norway, about 50\%.

Moreover, the recovery in enrolment rates by age 21 is marginal in Sweden: only up to some $41 \%$ (Table 2.1d). In Finland, on the other hand, the share of full-time students has by age 21 increased to much the same level as in Norway (a student share of about one-half in both countries). Hence, in this respect Sweden ranks far behind Finland and Norway and is also outstripped by Denmark. 
Figure 2.1d: Sweden: Average distribution (\%-share) of young people across main activities up to age 31, based on pooled information on all three youth cohorts

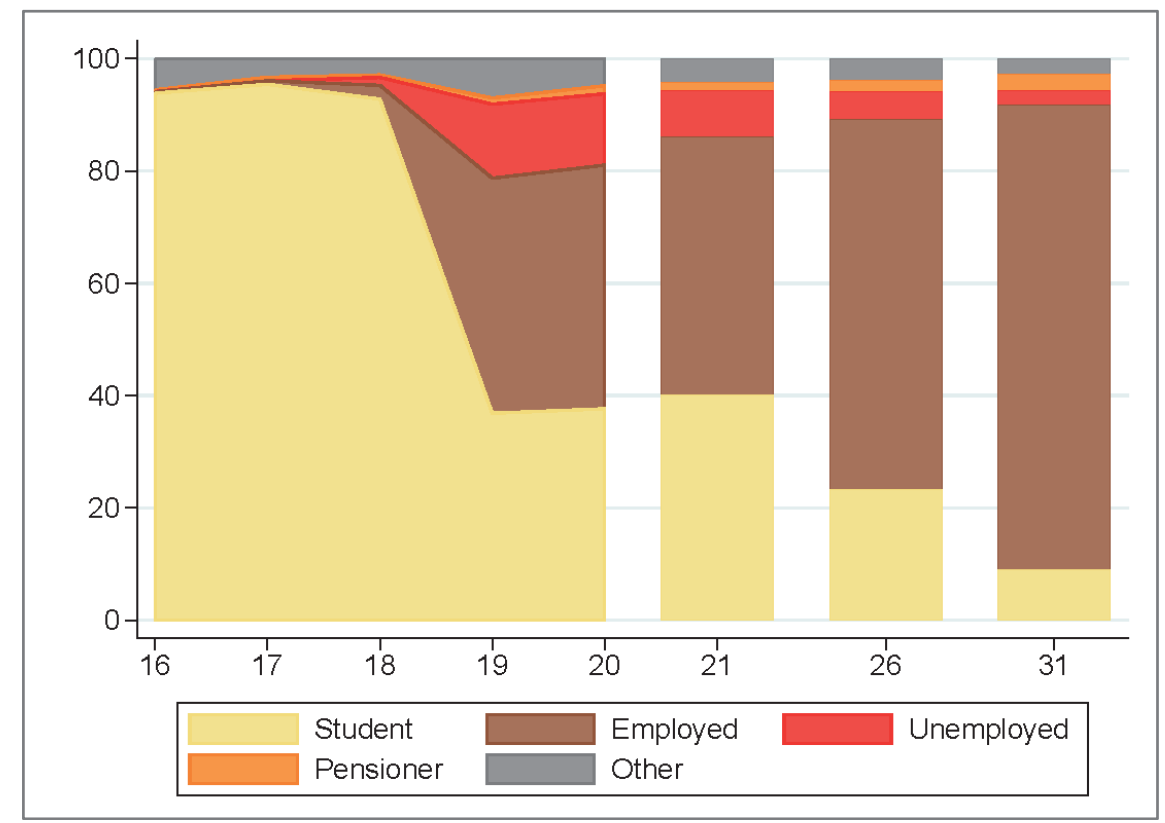

Note: For definitions of the five main activities, see sub-chapter 2.1.

Table 2.1d: Sweden: Average distribution (\%-share) of young people across main activities at age 21, based on pooled information on all three youth cohorts (all and by gender) and separately by cohort

\begin{tabular}{|c|c|c|c|c|c|c|}
\hline \multirow[t]{2}{*}{ Activity } & \multicolumn{3}{|c|}{ All three cohorts pooled } & \multicolumn{3}{|c|}{ By cohort of 16 -year-olds } \\
\hline & All & Males & Females & 1993 & 1998 & 2003 \\
\hline Full-time student & 40.2 & 33.9 & 46.9 & 41.4 & 42.6 & 37.1 \\
\hline Employed & 44.9 & 49.9 & 39.5 & 42.6 & 43.0 & 47.7 \\
\hline Unemployed & 9.2 & 10.4 & 7.8 & 11.8 & 9.2 & 6.9 \\
\hline Pensioner (disability benefits) & 1.6 & 1.7 & 1.4 & 0.9 & 1.4 & 2.3 \\
\hline Other (inactivity) & 4.2 & 4.1 & 4.3 & 2.3 & 3.9 & 6.0 \\
\hline Total & 100.0 & 100.0 & 100.0 & 100.0 & 100.0 & 100.0 \\
\hline$\%$-share in the pooled dataset & 100.0 & 51.6 & 48.4 & 31.2 & 31.9 & 36.9 \\
\hline
\end{tabular}

Note: For definitions of the five main activities, see sub-chapter 2.1.

Another feature that Sweden shares with both Finland and Norway is a notable increase at age 19 in activities outside education and working life. However, while the NEET activities at this particular age are in Norway dominated by "other" (unknown activities), the overbearing activity is unemployment for Finnish and even more so for Swedish youngsters: the share of young Swedes having registered as unemployed jobseekers is about $13 \%$ at age 19 and also at age 20. Although it drops to about $9 \%$ by age 21 , this is still the highest share of young unemployed among the four Nordic countries under study. Also the share of 
young people on disability benefits is relatively high among young Swedes, whereas the share in the dumping category "other" is much lower than in the other three countries.

However, a comparison of the average distribution across main activities in the three youth cohorts reveals that many of the peculiarities observed for Sweden (in Figure 2.1d) stem from occasionally quite remarkable changes over cohorts. Table $2.1 \mathrm{~d}$ highlights these cross-cohort differences at age 21 . As is evident from the table, the share of full-time students at age 21 is throughout rather low compared to the other three Nordic countries and has declined further in the youngest (2003) cohort. Simultaneously, the share in employment has increased over cohorts with almost one-half of the youngest cohort working by age 21 . However, these changes in studying and working shares concern only the youngest cohort, with these two shares being more or less the same for the 1993 and 1998 cohorts. The cross-cohort increase in employment is accompanied by a decline in unemployment, but also by a steady growth in the share on disability arrangements or in other types of inactivity. Almost one-half of young Swedes on disability benefits are estimated to be so severely disabled that they will never be able to take an ordinary job. Moreover, the Swedish Social Insurance Agency predicts that there will be a further increase over the next few years in the number of young people going into disability arrangements. This increase over time in the number of youth on disability benefits has received much attention in the political debate in Sweden.

As in the other Nordic countries, young women continue in full-time education to a much larger extent than do young men (Table 2.1d). The share of 21-year-old females enrolled in full-time education is close to that of their Danish counterparts, but notably lower than in Finland and Norway. The share of 21-year-old males studying on a full-time basis is extremely low, but counteracted by an employment share that well exceeds those observed for the other three countries. While also the share of working 21-year-old females is relatively high in Sweden, it is nonetheless more in line with what is observed for the other countries and, again, quite close to the corresponding share for Denmark. The differences in gender distributions across main activities are much smaller when it comes to NEET activities. As in the other three countries, the share among 21-year-old men on disability benefits is slightly larger (except in Norway) and the share in other types of inactivity slightly lower, when compared to the corresponding shares among 21-year-old females.

After age 21, the average distribution of young Swedes across the five main activity categories turns increasingly similar to the distributions 
observed for the other three countries. In particular, the share of young Swedes enrolled in full-time education is down at $23 \%$ by age 26 and drops to $9 \%$ by age 31 . These shares are very close to those observed for Denmark and Finland, and especially for Norway. Likewise, the share of young people in employment has increased to about $65 \%$ by age 26 , and to as much as $82 \%$ by age 31 . Concomitantly, there is a striking decline in the share of young people in unemployment. While the share on disability benefits increases slightly up to age 31 , there is a further decline in the share of young people in other types of inactivity.

\section{Box 2.5 Sweden- a brief outline of the relevant institutional framework}

\section{Upper secondary school system}

Education in Sweden is compulsory from the autumn term of the year the child turns 7 and lasts for nine years, until the spring term after grade 9. All tiers of schooling are a municipal responsibility regulated by the 1985 Education Act (Ministry of Education and Research, 2000) and overseen by the Swedish National Agency for Education. After completed compulsory education, every young person has a statutory right to attend three years of upper secondary education free of charge. Upper secondary school is formally elective, although most attend it. When applying for upper secondary education, the youth may choose between two main tracks: academic and vocational studies both of which last for three years. Around two-thirds of the students opt for the academic track, which prepares for further studies in universities and colleges. The rest of the youth start a vocational study program, which gives qualifications for work in a number of different occupations. Women and those living in the larger city areas tend to choose academic tracks to a larger degree while men and those living in more sparsely populated areas tend to choose the vocational tracks to a larger degree.

Students apply to upper secondary school based on their grades from compulsory school. Students generally attend a school in their municipality of residence, but if the desired track is not offered they can instead choose to attend a school in a nearby municipality.

An educational reform in 1991 introduced a more comprehensive upper secondary school system in Sweden which substantially reduced the differences in curricula between the academic and vocational tracks in upper secondary school. This was done by considerably increasing the academic content of all vocational tracks. The length of these tracks was at the same time extended from two to three years, giving them the same length as the academic tracks. At the same time, students graduating from a vocational track attained basic eligibility for university studies. 
With the 2011 reform of upper secondary school, several changes have been made in vocational education. The largest change was the introduction of apprenticeship training. The reform also reduced the scheduled time for the compulsory theoretical subjects. In an international perspective, the Swedish system for vocational education can still be characterized as being academically orientated both in terms of large amounts of theory in the school-based part of the education and the relatively small share of work place-based learning. The required amount of work place-based learning is 15 weeks during the three years of the program, which is less than in most other countries.

\section{Measures directed towards young people outside education and the} labour market

The youth guarantee and active labour market programs: Young people aged 16 to 24 , who have recently left the education system and have not yet found a job are assured participation in an active labour market program through the Youth Guarantee (Jobbgaranti för ungdomar). Participation is full time unless there are specific circumstances such as illness, disability, parental leave or some other reason accepted by the employment agency. The youth guarantee was introduced in 2007. The purpose of the youth guarantee is to offer specific labour market support at an early stage. Examples of support are education, job search assistance and practical training or an internship. The Youth Guarantee replaced the earlier youth guarantee program (Utvecklingsgaranti för ungdomar), which was introduced in 1998 and targeted at unemployed persons aged 20-24. The guarantee included an assignment to some labour market program within 100 days of unemployment.

Unemployment benefits: The unemployment insurance scheme in Sweden is based on previous income. To receive income-related benefits, one must have been a member of an unemployment insurance fund for at least twelve months (membership condition). The person must also have satisfied a work condition. The requirement is that one must have worked at least 80 hours a month for at least six months during the last twelve months before becoming unemployed.

Young school-leavers do in general not qualify for unemployment benefits, as they do not fulfil the income requirements. This could be expected to lower their incentives to register as unemployed. However, participation in some youth labour market programs such as job training (Arbetslivsintroduktion) and the Youth Guarantee (Jobbgaranti för ungdomar) qualifies for payment at a low level (utvecklingsersättning). 
Social assistance: Young people (18 years and above) who are not able to financially support themselves through employment or other means may receive social assistance. The benefit is temporary, and the aim is to secure financial independence. In order to be entitled to receive social assistance, all other options must have been considered. This includes gainful employment, own savings and other financial rights, such as unemployment benefits. The right to social assistance is not based on the income principle but on individual needs assessment, that is, the amount received is means-based, and each case is specifically and individually assessed. This means that it is possible to receive social assistance as a supplement to other income, if this income is too low to cover basic subsistence costs. Often there are certain conditions attached to the entitlement of social assistance, but these conditions are always set on an individual basis. For instance, non-employed individuals may have to register as jobseekers or participate in an active labour market program in order to receive the benefit. Among the adult social assistance receivers, 39\% were between 18-29 years old (Socialstyrelsen 2014).

Disability benefits: In 2003, the earlier system for early retirement (förtidspension) was replaced by aktivitetsersättning for individuals between 19 and 29 years-of-age. The purpose of the reform was to reduce the number of young people on disability benefits and facilitate their return to work through targeted activities. Before 2003, the disability insurance was part of the old public pension system, and from 2003, it became part of the social insurance system. The reason for this change was to come up with a financially cohesive insurance scheme that included all benefits that compensate income losses for people with reduced work ability. In 2003, the cooperation between the Social insurance agency and the Employment services was expanded, primarily in order to improve the ability of long-term sick to return to work.

According to current regulations, youth who have reduced work capacity due to physical or mental health problems may be eligible for disability benefits. If the employees are between 19 and 29 years old, they may receive benefits (aktivitetsersätting) provided that their work capacity is reduced by at least a quarter for at least one year. Compensation is limited in time and granted for a maximum of three years each. During the compensation period there is a plan to return to work. The plan allows the employer to be one of the actors who will enable employees to return to work. During the time that the employee receives benefits, he or she has the opportunity to participate in activities that have a positive impact on the disease condition or performance. The activity also aims to improve work capacity. 


\subsubsection{Main findings}

This sub-chapter has given a general picture of the average changes occurring in young people's post-compulsory-school activities on their way through education and to the labour market. As discussed above when commenting on the patterns observed for each of the four Nordic countries under study, the overall picture looks more or less the same: declining shares in full-time education and rapidly growing shares in employment when the young people in our three cohorts grow older. In other words, large shares of young people are rapidly and successfully integrated into the labour market. However, in all four countries there are also non-negligible shares of young people experiencing unemployment, encountering health problems moving them onto disability benefits, or engaging in other types of inactivity outside both education and work.

Despite of sharing this overall pattern, there are also distinct differences between the four countries, especially when it comes to full-time studies and employment. Although much attention is paid to these differences in the above country-specific sections, there is nonetheless reason to recall the most conspicuous divergences with the help of Table 2.1e, which repeats the activity shares at age 21 as calculated from pooled information on all three youth cohorts. In particular, we have observed low shares of 21-year-olds enrolled in full-time education for Denmark and Sweden accompanied by relatively high shares of 21-year-olds in working life. At the other extreme we have Finland and Norway with high shares of young people studying on a full-time basis still at age 21 , whereas their share in employment is relatively low. However, when adding up these two "activity" shares, as in Table 2.1e, the differences across countries almost disappear.

\begin{tabular}{|c|c|c|c|c|}
\hline \multirow[t]{2}{*}{ Activity at age 21} & \multicolumn{4}{|c|}{ All three cohorts merged } \\
\hline & Denmark & Finland & Norway & Sweden \\
\hline Full-time student & 43.7 & 49.0 & 50.1 & 40.2 \\
\hline Employed & 43.2 & 35.6 & 34.0 & 44.9 \\
\hline Activity share (full-time student + employed) & 86.9 & 84.6 & 84.1 & 85.1 \\
\hline Unemployed & 3.9 & 8.6 & 5.4 & 9.2 \\
\hline Pensioner (disability benefits) & 0.7 & 1.0 & 1.4 & 1.6 \\
\hline Other (inactivity) & 8.5 & 5.7 & 9.1 & 4.2 \\
\hline NEET share (unemployed + pensioner + other) & 13.1 & 15.3 & 15.9 & 15.0 \\
\hline Total & 100.0 & 100.0 & 100.0 & 100.0 \\
\hline
\end{tabular}

Note: For definitions of the five main activities, see sub-chapter 2.1. 
When moving beyond the age of 21, however, the shares of young people in full-time education and in employment turn increasingly similar in the four countries. In other words, the cross-country differences in studying and working shares observed over the five years following completion of compulsory education shrink notably in adulthood but do not disappear, as will also be shown in Chapter 6. This notion points to a relatively strong influence of especially upper secondary education systems but also of labour market institutions on the early school-to-work-transition patterns of young people leaving compulsory school and, ultimately, also on labour market outcomes in adulthood. We return to this question in Chapter 6 and, especially, in Chapter 7.

The counterpart to the cross-Nordic similarity in activity (education + employment) shares among 21-year-olds is NEET shares of approximately the same magnitude (Table 2.1e). However, these NEET shares are found to conceal occasionally quite notable cross-country differences in the distribution of young people into unemployment, disability benefits and other types of inactivity: the dominating NEET activity among 21-year-olds is unemployment in Finland and Sweden but "other" (unknown activity) in Denmark and Norway. Hence, while crossNordic differences in upper secondary education systems and in labour market institutions seem to leave the NEET share largely unaffected, educational and institutional particularities do seem to influence the distribution of young people across alternative NEET activities and, as a consequence, labour market outcomes as a young adult. Also this aspect is investigated in more depth in Chapters 6 and 7.

\subsection{Non-completers - an introductory view}

The previous sub-chapter has shown that large shares of young people continue directly in post-compulsory education, complete an upper secondary degree and move into tertiary-level education, or are successfully integrated into the labour market. There are, however, also young people facing serious problems when it comes to post-compulsory education and labour market integration. A non-negligible share of young people fails to achieve an upper secondary certificate with often detrimental influence on their labour market prospects. In this sub-chapter, we take a closer look at this non-completion phenomenon in the four Nordic countries under study. 


\subsubsection{Non-completion at age 21}

Upper secondary education aims to provide students with the basic skills and knowledge needed to successfully enter the labour market, or higher education. It is by now a stylized fact that completing upper secondary education has a positive effect on later outcomes in terms of better labour market prospects. Accordingly, most countries make considerable efforts to improve upper secondary graduation rates for all students.

\section{Box 2.6 Definition of "non-completer"}

In our analysis, we define non-completion as not having completed upper secondary education by the year one turns 21 years-of-age. Instead of using the term "drop-out" or "early school leaver", which in this case would be somewhat misleading, we prefer to use the term "non-completer" for those who still five years after completed compulsory education have no upper secondary degree. In other words, their only formal education by age 21 is primary education.

Table 2.2 presents country-specific shares of non-completers for the three youth cohorts under scrutiny, with the non-completer defined as a young person who has not finished upper secondary education by the age of 21 (cf. Box 2.6). As is evident from the table, these non-completion shares are highly similar for Finland and Sweden, ranging between 14 and 20 per cent depending on the youth cohort in question. The non-completion shares for these two countries also reveal a strikingly similar trend over cohorts: a growing share from the 1993 cohort to the 1998 cohort, followed by a slight decline for the youngest (2003) cohort. In both countries, however, the share of 21-year-old non-completers remains at a higher level in the youngest cohort as compared to the oldest cohort.

\begin{tabular}{|c|c|c|c|c|}
\hline Cohort & Denmark & Finland & Norway & Sweden \\
\hline 16-year-olds in 1993 & 34.7 & 16.0 & 28.5 & 14.5 \\
\hline 16-year-olds in 1998 & 39.0 & 19.7 & 29.1 & 17.0 \\
\hline 16-year-olds in 2003 & 38.3 & 18.4 & 31.5 & 16.5 \\
\hline
\end{tabular}

The situation looks different in Denmark and Norway. While these two countries are characterised by notably higher non-completion shares among 21-year-olds, when compared to Finland and Sweden, the development of these shares across cohorts reveals more differences than similarities between the two countries. In Norway, the non-completion rate was of much the same magnitude in the 1993 and 1998 cohorts but increased further in the 2003 cohort. Hence, the share of non- 
completers in the youngest cohort was almost twice as large in Norway as in Sweden.

The highest non-completion rate among 21-year-olds is obtained for Denmark. As for the other Nordic countries under study, it increased between the oldest cohort and the middle cohort, but clearly more than in the other three countries. In the 1998 cohort, only three out of five had completed an upper secondary degree by age 21 . The noncompletion rate was only marginally lower in the youngest cohort.

Due to broad political agreement in the Nordic countries on the desirability of investing in education and training throughout working life, current policies promote also later educational initiatives, both within the framework of the secondary education system and in the adult education system. Therefore, it is not uncommon that individuals who drop out of school re-enter, sooner or later, the education system to finish their upper secondary education. This is also evident in the next figures which show, separately for the four countries, the share of noncompleters in our three youth cohorts, from age 19 up to age 31. The non-completion shares at age 21 are, of course, identical to those displayed in Table 2.2 above. Additionally we present the evolution of noncompletion shares also by gender.

\subsubsection{Non-completion beyond age 21: Denmark}

Figure 2.2a shows that the remarkably high share of non-completers observed among 21-year-old Danes shrinks rapidly up to age 31 (upper graph). In the oldest (1993) cohort, it declines from having been about $35 \%$ at age 21 to $17 \%$ by age 31 . A similar trend is discernible for the middle (1998) cohort, except that the share of non-completers starts out from a higher level and also remains at a higher level: 23\% among 26year-olds compared to $21 \%$ in the oldest cohort. On the whole, this implies that large shares of young Danes prolong their studies in upper secondary education and that this tendency has strengthened over cohorts.

A split by gender (lower graph of Figure 2.2a) reveals, in turn, that the non-completion share is much higher among young men than among young women. In other words, larger shares of young women complete a post-compulsory degree and, moreover, at a faster pace (i.e. younger age). Additionally the graph indicates that the gender gap in noncompletion shares has widened over cohorts, mainly due to increasing shares of non-completers among young men. At age 26, for instance, this gap was 5.5 percentage points in the 1998 cohort compared to 3.3 per- 
centage points in the 1993 cohort. Even larger gender gaps, and changes in these gaps, are observed at younger ages, as illustrated in Table 2.3a.

Figure 2.2a: Denmark: Non-completion rates (\%-shares) at different ages, by cohort (upper graph) and by cohort and gender (lower graph)

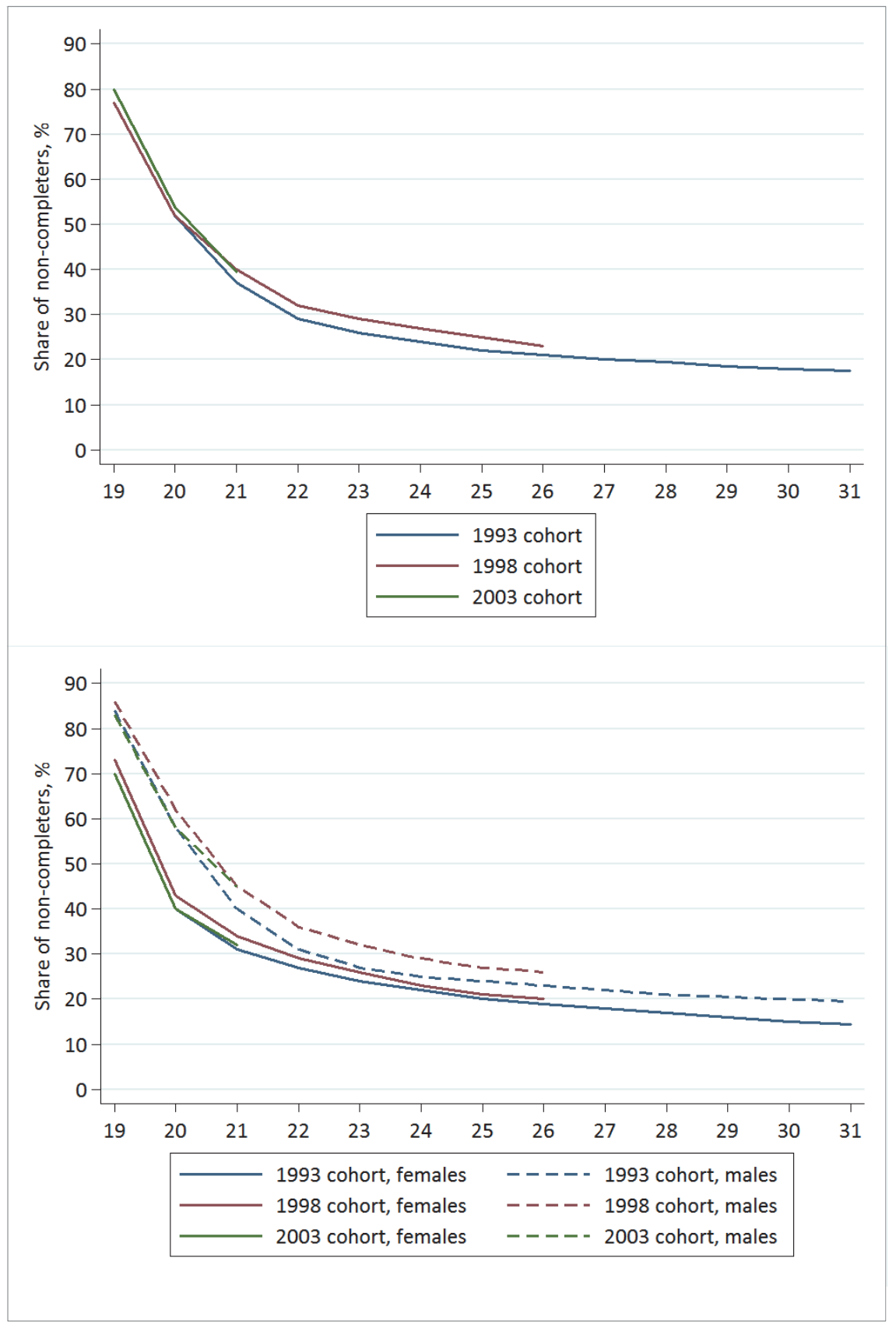




\begin{tabular}{|c|c|c|c|c|c|c|}
\hline \multirow[b]{2}{*}{ Cohort } & \multicolumn{2}{|c|}{ At age 21} & \multicolumn{2}{|c|}{ At age 26} & \multicolumn{2}{|c|}{ At age 31} \\
\hline & $\begin{array}{r}\text { Young } \\
\text { men }\end{array}$ & $\begin{array}{r}\text { Young } \\
\text { women }\end{array}$ & $\begin{array}{r}\text { Young } \\
\text { men }\end{array}$ & $\begin{array}{r}\text { Young } \\
\text { women }\end{array}$ & $\begin{array}{c}\text { Young } \\
\text { men }\end{array}$ & $\begin{array}{r}\text { Young } \\
\text { women }\end{array}$ \\
\hline 16-year-olds in 1993 & 39.2 & 30.3 & 22.1 & 18.8 & 19.2 & 14.4 \\
\hline 16-year-olds in 1998 & 43.9 & 34.3 & 25.3 & 19.8 & n.a & n.a \\
\hline 16-year-olds in 2003 & 44.6 & 32.1 & n.a & n.a & n.a & n.a \\
\hline
\end{tabular}

Note: n.a. = information not available in the data.

\subsubsection{Non-completion beyond age 21: Finland}

The corresponding information for Finland is provided in Figure 2.2b. By and large, the overall pattern resembles that observed for Denmark. In particular, the non-completion share in the 1998 cohort starts out from a higher level and also remains at a higher level, when compared to the situation of the oldest (1993) cohort. There is, however, one distinct difference between Finland and Denmark: in Finland, the share of noncompleters is quite low already at age 21 and it declines only marginally beyond this age. In the oldest cohort, it shrinks from $16 \%$ at age 21 to below $12 \%$ at age 26 and $10 \%$ at age 31 . Likewise, in the middle (1998) cohort it is down at about $13 \%$ at age 26 compared to almost $20 \%$ at age 21 . Hence, not much seems to happen to young Finns' non-completion rates beyond age 21, a conclusion also drawn by e.g. Myrskylä (2011b).

The lower graph of Figure $2.2 \mathrm{~b}$ shows that non-completion is a more typical phenomenon among young men than among young women also in Finland. Moreover, the gender gap in non-completion shares turns out to widen remarkably at age 20 , but remains thereafter approximately unchanged. And in contrast to the situation in Denmark, there seems to be only marginal changes in the gender non-completion gap over cohorts, as is also evident from Table $2.3 \mathrm{~b}$. 
Figure 2.2b: Finland: Non-completion rates (\%-shares) at different ages, by cohort (upper graph) and by cohort and gender (lower graph)

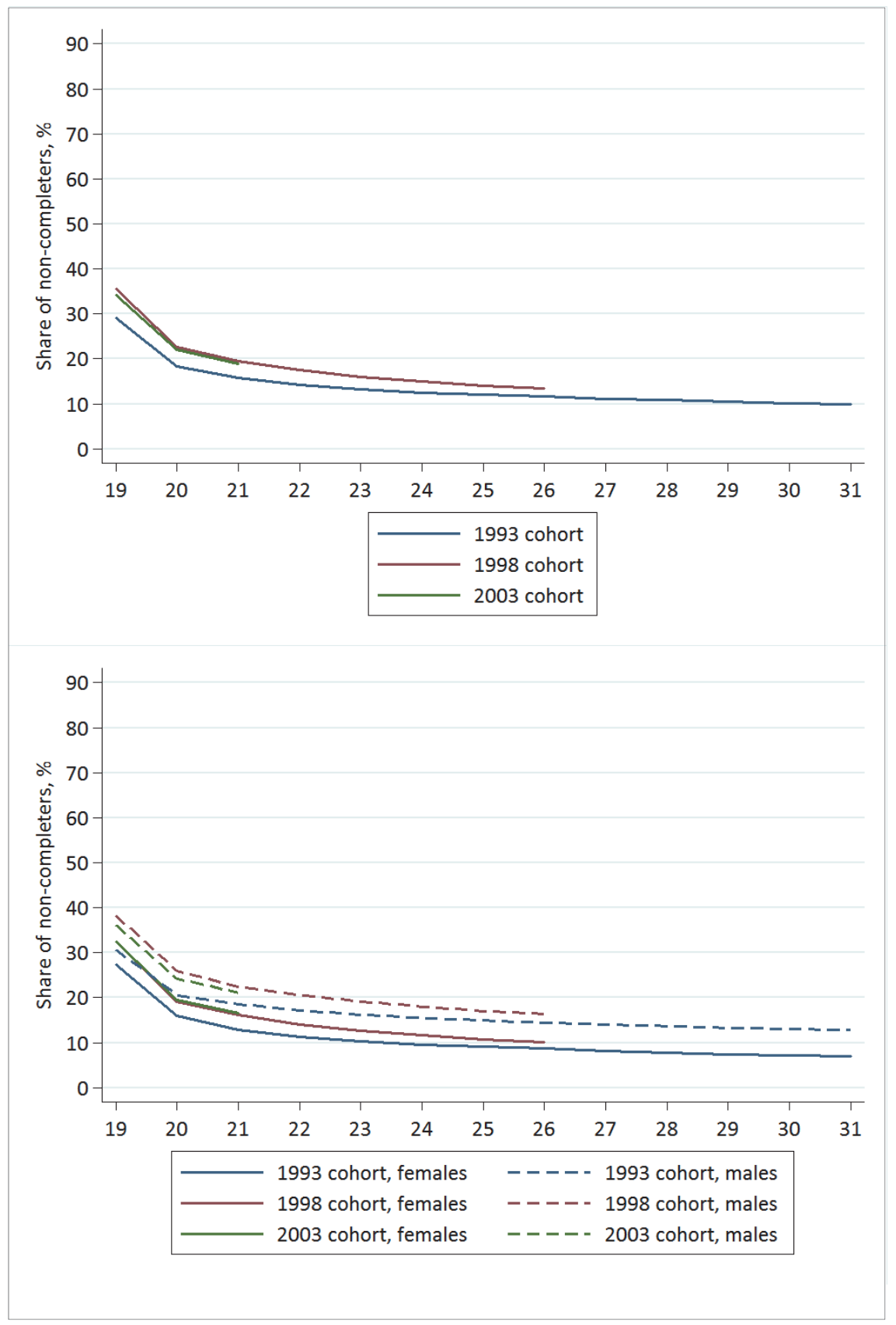




\begin{tabular}{|c|c|c|c|c|c|c|}
\hline \multirow[b]{2}{*}{ Cohort } & \multicolumn{2}{|c|}{ At age 21} & \multicolumn{2}{|c|}{ At age 26} & \multicolumn{2}{|c|}{ At age 31} \\
\hline & $\begin{array}{r}\text { Young } \\
\text { men }\end{array}$ & $\begin{array}{r}\text { Young } \\
\text { women }\end{array}$ & $\begin{array}{r}\text { Young } \\
\text { men }\end{array}$ & $\begin{array}{r}\text { Young } \\
\text { women }\end{array}$ & $\begin{array}{c}\text { Young } \\
\text { men }\end{array}$ & $\begin{array}{r}\text { Young } \\
\text { women }\end{array}$ \\
\hline 16-year-olds in 1993 & 18.6 & 12.9 & 14.5 & 8.7 & 12.8 & 6.9 \\
\hline 16-year-olds in 1998 & 22.4 & 16.1 & 16.4 & 10.1 & n.a. & n.a. \\
\hline 16-year-olds in 2003 & 21.1 & 16.5 & n.a. & n.a. & n.a. & n.a. \\
\hline
\end{tabular}

Note: n.a. = information not available in the data.

\subsubsection{Non-completion beyond age 21: Norway}

As shown in Table 2.2 above, the share of non-completers among 21year-old Norwegians has remained at a relatively high level over cohorts, albeit clearly below those observed for Denmark. However, the non-completion rate declines at a steady pace after age 21 . In the oldest cohort, it is by age 26 down at $22 \%$ and by age 31 at $20 \%$ (Figure $2.2 \mathrm{c}$, upper graph). Moreover, the non-completion rate beyond age 21 evolves in much the same manner for the 1998 cohort, implying small, if any, changes across cohorts in this respect. Notably larger changes across cohorts are observed prior to age 21 with the delay in completion rates at these younger ages showing a steady growth across cohorts.

Figure 2.2c: Norway: Non-completion rates (\%-shares) at different ages, by cohort (upper graph) and by cohort and gender (lower graph)

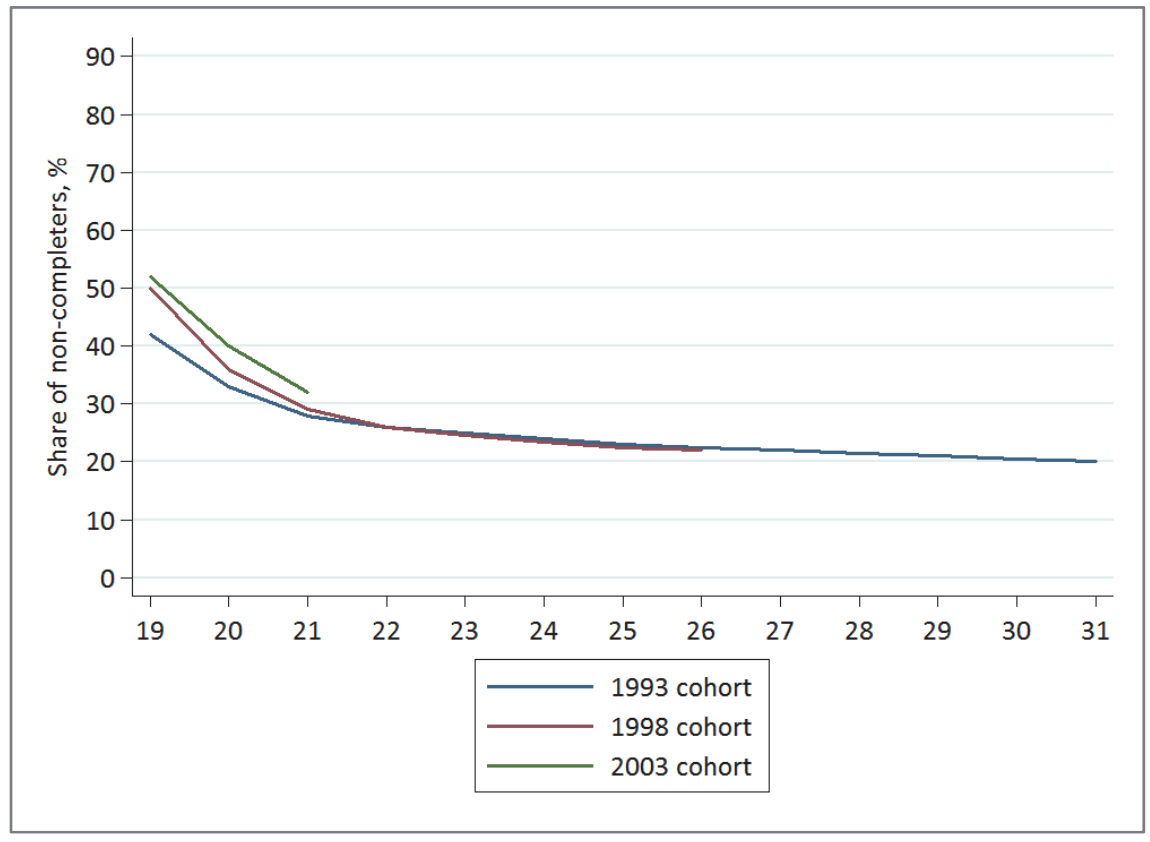


Figure 2.2c continued

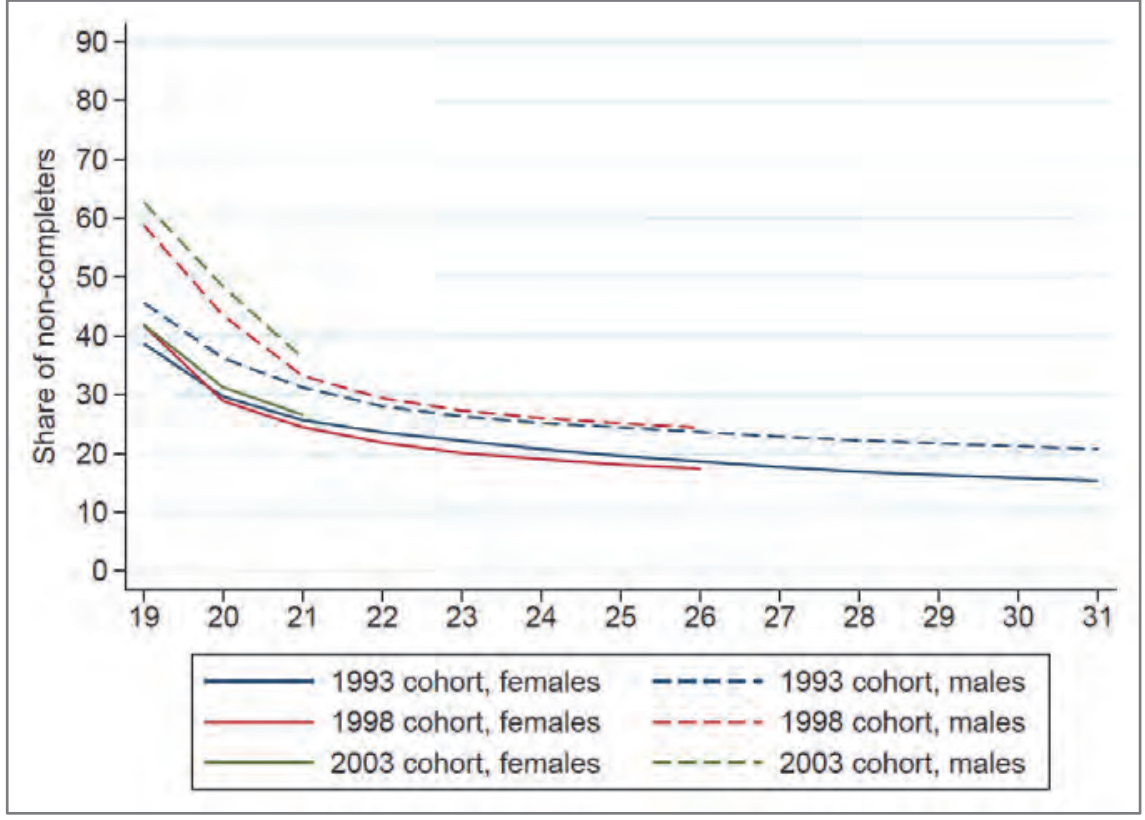

The lower graph of Figure 2.2c provides at least part of an explanation for the changes in early non-completion shares observed across the three Norwegian youth cohorts. First, non-completion rates were much higher among young men than among young women already in the oldest cohort, but this gender gap in non-completion shares did not change much with age: it was about 7 percentage points at age 19, and of a similar size also at age 21 and still some ten years later, at age 31 (cf. Table 2.3c). Second, the share of non-completers among young women has changed only marginally across cohorts. Among young men, in contrast, we observe a marked delay in completion rates, especially below age 21 , a delay that seems to have strengthened further in the youngest cohort. This pronounced difference in early non-completion rates for young men belonging to the three youth cohorts under scrutiny is likely to be related to the fundamental reform of the Norwegian upper secondary school system in 1994 (Reform 94), which lengthened the standard duration of most vocational courses from three to four years (cf. Box 2.4 
above). As young men are overrepresented in vocational programs, this may well explain the delay in completion rates observed for boys belonging to the 1998 and 2003 cohorts of 16-year-olds.

\begin{tabular}{|c|c|c|c|c|c|c|}
\hline \multirow[b]{2}{*}{ Cohort } & \multicolumn{2}{|c|}{ At age 21} & \multicolumn{2}{|c|}{ At age 26} & \multicolumn{2}{|c|}{ At age 31} \\
\hline & $\begin{array}{r}\text { Young } \\
\text { men }\end{array}$ & $\begin{array}{r}\text { Young } \\
\text { women }\end{array}$ & $\begin{array}{c}\text { Young } \\
\text { men }\end{array}$ & $\begin{array}{r}\text { Young } \\
\text { women }\end{array}$ & $\begin{array}{c}\text { Young } \\
\text { men }\end{array}$ & $\begin{array}{r}\text { Young } \\
\text { women }\end{array}$ \\
\hline 16-year-olds in 1993 & 31 & 26 & 24 & 19 & 21 & 15 \\
\hline 16-year-olds in 1998 & 33 & 24 & 24 & 17 & n.a. & n.a. \\
\hline 16-year-olds in 2003 & 36 & 27 & n.a. & n.a. & n.a. & n.a. \\
\hline
\end{tabular}

Note: n.a. = information not available in the data.

\subsubsection{Non-completion beyond age 21: Sweden}

The lowest non-completion rates among 21-year-olds are observed for Sweden (cf. Table 2.2 above). Moreover, as in Finland, they do not change much after age 21 (Figure $2.2 \mathrm{~d}$, upper graph). In the oldest cohort, we see a decline from $14.5 \%$ at age 21 to $12 \%$ at age 26 and $11 \%$ at age 31. A similar evolution is discernible in the two younger cohorts, albeit at non-completion rates of a slightly higher magnitude.

A comparison of non-completion rates across genders indicates that young men fair worse than young women also in Sweden (Figure 2.2d, lower graph). Moreover, the gender gap in non-completion rates increases over time in two crucial respects. First, while the gap is about 3 percentage points at age 21 , it increases to 4.5 percentage points by age 31 in the oldest (1993) cohort. Second, the gender gap in noncompletion rates shows an increasing trend across cohorts. In the middle (1998) cohort, it is as high as 5 percentage points at age 26 , which means a doubling compared to the situation at age 21 (a gap of 2.5 percentage points). A still higher gender gap in non-completion shares at age 21 is observed for the youngest cohort (cf. Table 2.3d). 
Figure 2.2d: Sweden: Non-completion rates (\%-shares) at different ages, by cohort (upper graph) and by cohort and gender (lower graph)

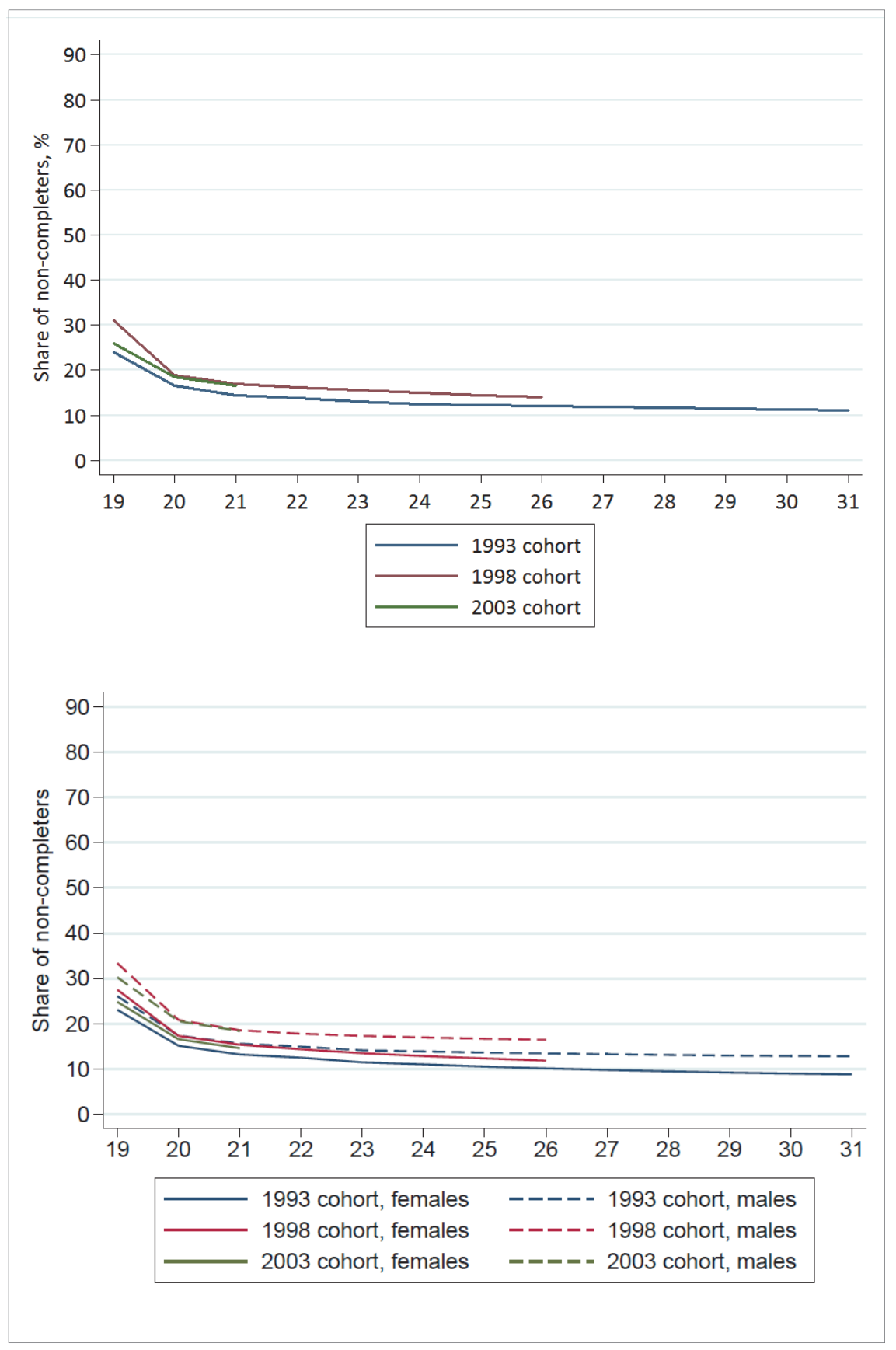




\begin{tabular}{|c|c|c|c|c|c|c|}
\hline \multirow[b]{2}{*}{ Cohort } & \multicolumn{2}{|c|}{ At age 21} & \multicolumn{2}{|c|}{ At age 26} & \multicolumn{2}{|c|}{ At age 31} \\
\hline & $\begin{array}{c}\text { Young } \\
\text { men }\end{array}$ & $\begin{array}{r}\text { Young } \\
\text { women }\end{array}$ & $\begin{array}{r}\text { Young } \\
\text { men }\end{array}$ & $\begin{array}{r}\text { Young } \\
\text { women }\end{array}$ & $\begin{array}{r}\text { Young } \\
\text { men }\end{array}$ & $\begin{array}{r}\text { Young } \\
\text { women }\end{array}$ \\
\hline 16-year-olds in 1993 & 16 & 13 & 13 & 10 & 13 & 8.5 \\
\hline 16-year-olds in 1998 & 18 & 15.5 & 17 & 12 & n.a. & n.a. \\
\hline 16-year-olds in 2003 & 18 & 14.5 & n.a. & n.a. & n.a. & n.a. \\
\hline
\end{tabular}

Note: n.a. = information not available in the data.

\subsubsection{Main findings}

In this sub-chapter, we have explored the prevalence of non-completion of an upper secondary degree in our three country-specific youth cohorts. We notice that the share of non-completers among 21-year-olds varies considerably across the four Nordic countries under study. At this particular age, it is remarkably high in Denmark and comparatively low in both Finland and Sweden. Norway falls in between but is much closer to Denmark than to Finland or Sweden. After age 21, the share of noncompleters starts declining in all four countries, an aspect that we will return to in Chapter 6. However, the cross-Nordic differences in noncompletion rates do not disappear despite a declining non-completion rate with age: Finland and Sweden have the lowest non-completion rates (10-11\%) also among the 31-year olds, compared to $17 \%$ in Denmark and $20 \%$ in Norway. Our analysis reported in Chapter 6 shows that these differences are not trivial when it comes to the labour market prospects of young adults.

From a cross-cohort perspective, the four Nordic countries share the feature of younger cohorts revealing higher shares of non-completers among the 21-year-olds. In other words, there is a clear trend towards delayed graduation from upper secondary school among Nordic youth. Moreover, this growth in non-completion rates across cohorts is largely retained also beyond the age of 21 . Put differently, a higher share of noncompleters among 21-year-olds typically results in a higher share of non-completers also at later ages, say, among 26-year-olds. However, a conspicuous exception to this pattern observed across cohorts is Norway showing remarkable delays over time in completion rates among notably 19- and 20-year-olds (a trend restricted to young men, though), whereas the cross-cohort differences in non-completion rates are minor beyond the age of 20 .

We have also shown that much of these findings for the full cohorts can be explained by notable differences between young men and young women in upper secondary completion rates. In all four countries, we 
observe lower non-completion rates for young women. The gender gaps in non-completion rates tend to be rather small up to age 21, though, and have changed only marginally over the three cohorts under scrutiny, except for Norway where there has been a conspicuous increase in noncompletion rates among 19- to 20 -year-old males belonging to younger cohorts. In some of the countries, however, this gender gap in noncompletion rates reveals an increasing trend beyond age 21 , a trend that, moreover, seems to have strengthened over cohorts, again mainly due to a worsening situation among young men. This holds true for Denmark and especially for Sweden. For the other two countries, we observe only a small (Norway) or even negligible (Finland) change over cohorts in the gender gap in non-completion rates beyond age 21 .

Finally, we have also reason to contrast our non-completion rates to those reported elsewhere. The Norwegian rates are comparable to those reported by Falch and Nyhus (2009) and Bratsberg et al. (2010) while the Danish rates are comparable to those reported by Jakobsen and Liversage (2010). Our findings are also comparable to those of Bäckman et al. (2011), who compare dropout rates across the Nordic countries, measured seven years after the school start. Our shares of noncompletion rates are also in line with the descriptive information published by international bodies, notably Eurostat and OECD. For example, the high expected upper secondary completion rates reported by the OECD (see Table 1.4 of Chapter 1) reflect the fact that a sizeable fraction of young non-completers continues in education on a full-time basis, and eventually completes an upper secondary degree only later on, that is, after age 21. As shown above, delayed completion of upper secondary education is quite common in Norway and especially in Denmark, but much less so in Finland and Sweden.

\subsection{Individual school-to-work profiles - a general picture}

So far, we have described how the overall distribution across main activities evolves when compulsory-school-leaving youths grow older. While providing interesting information per se, this general picture tells nothing about young people's individual experiences after completing compulsory school. In other words, based on this average pattern we are not able to learn how single individuals shift between activities on their way through the education system and to the labour market. Next we therefore drill one step down to explore what the individual school-to-work 
transitions look like. This is done by use of so-called sequence analysis. Accordingly, we start by explaining the basic idea of sequence analysis.

\subsubsection{Sequence analysis - a brief outline}

As already noted, the average shares of young people in different main activities, as displayed in sub-chapter 2.2 , cannot inform us about the school and labour market experiences of individual youngsters in these critical years following the completion of compulsory school. Instead we need to look into each young person's main activity in each year and, based on this information, construct for each of them an individual postcompulsory-school trajectory, that is, a sequence of annual main activities. This individual trajectory then shows in which activity the young person has mainly been engaged in each year investigated. We thereby obtain a sequence of main activities for each young person in our data. For our present purposes, we restrict these individual trajectories to cover the 5-year period immediately following completion of compulsory education, that is, from age 16 up to age 20 .

This idea on which our subsequent analysis is based can be illustrated by means of a simple example. Presume that three of our youth cohort members experience, from age 16 up to age 20, the following sequences of main activities:

- Sequence one:

- Sequence two: 11211

- Sequence three: 12544

The young person in sequence one is a full-time student (activity 1) at ages 16, 17 and 18, employed (activity 2) at age 19, and again a full-time student at age 20 . The person in sequence two is a full-time student at all ages except for age 18 when employed. The person in sequence three, finally, is a full-time student at age 16 , employed at age 17 , in the residual inactivity category "other" (activity 5) at age 18 and shows up as a pensioner (activity 4, on disability benefit) at age 19 and also at age 20 .

In reality, the potential combinations of activities and, hence, the possible number of individual sequences are evidently enormous and accordingly difficult to handle without the help of some specific technique for categorising them into a reasonable number of groups (clusters). We will return, in Chapter 3, to this clustering of individual sequences and the patterns obtained for our four Nordic countries. 
Here we next present, separately for each country, the individual post-compulsory-school trajectories of each young person in our three pooled youth cohorts, starting from their main activity at age 16 and ending with their main activity in the year they turned 20. Additionally, these sequences of main activities obtained for the full cohorts will be contrasted against those of non-completers, that is, young people whose only formal education by age 21 is primary school.

\subsubsection{Denmark: all vs. non-completers}

Figure 2.3a illustrates the individual trajectories of 16-year-old Danes up to age 20 when based on pooled information on all three youth cohorts under scrutiny. The upper graph highlights the situation for all young people in the three cohorts while the lower graph displays the individual trajectories of non-completers only. The y-axis of the upper graph reveals that, taken together, the three Danish youth cohorts cover a total of 164,879 young people. Accordingly, the graph gives the combined outcome of 164,879 individual trajectories, when ranked according to the length of each young person's unbroken record of years in education straight after leaving compulsory school: those with all years between age 16 and age 20 spent in full-time education appear highest up in the graph and those starting with a year outside education lowest down in the graph. Put differently, each row in the graph represents an individual trajectory and, when ranking all these individual sequences of activities over the ages 16 to 20 according to the number of the youngster's initial years spent in post-compulsory school, the outcome for the full cohorts is the situation illustrated in the upper graph of Figure 2.3a. Likewise, when restricting the individual trajectories to noncompleters only, the lower graph shows that the Danish data covers a total of 61,844 young people with only a basic education still at age 21 , and that the individual trajectories of these 61,844 young people add up to the situation illustrated in this graph. 
Figure 2.3a: Denmark: Individual school-to-work trajectories from age 16 up to age 20 for all young people (upper graph) and separately for non-completers (lower graph)

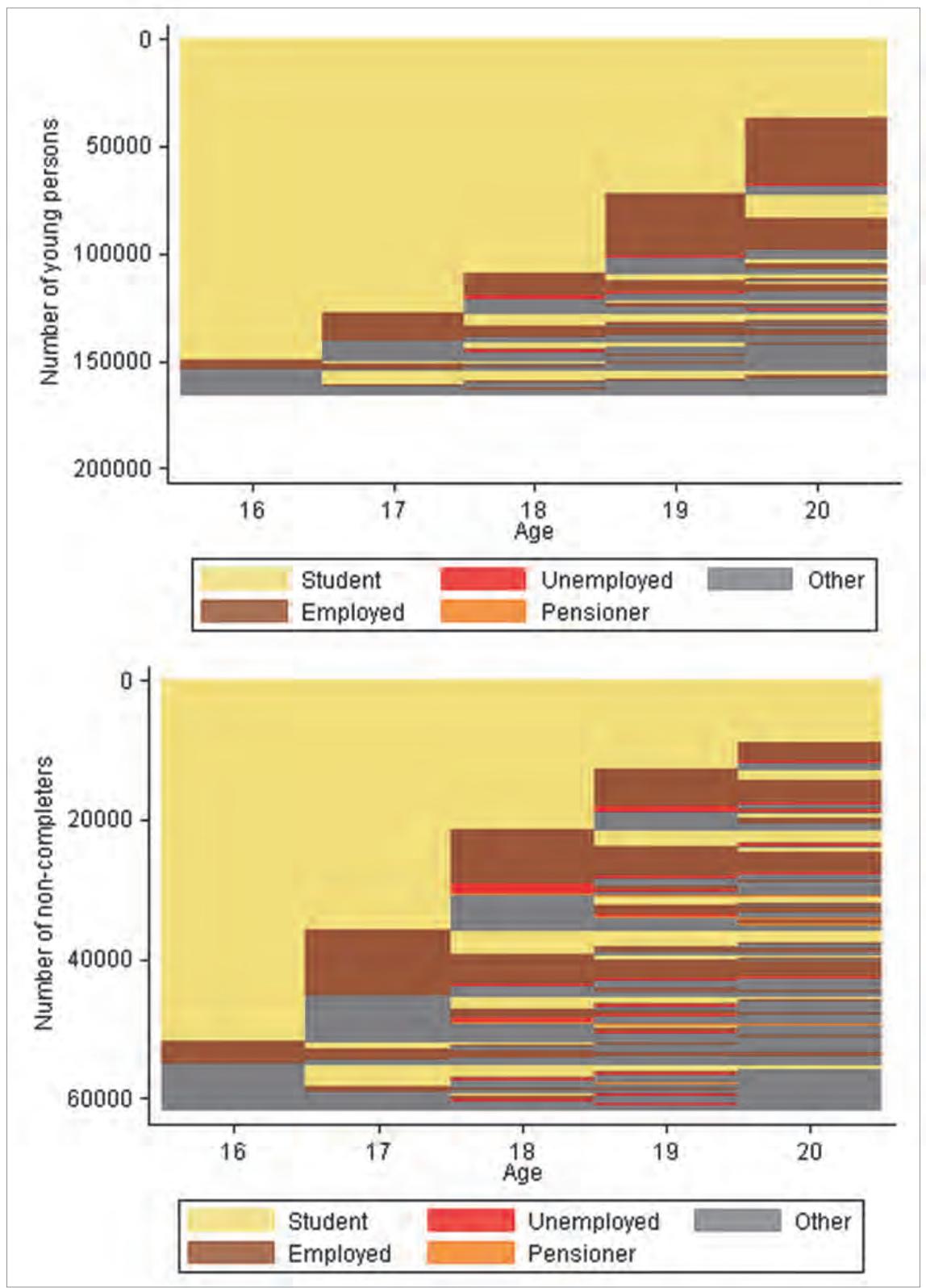

Notes: Both graphs are based on pooled information on all three youth cohorts under scrutiny. Noncompleters refer to young people who still five years after completing their compulsory education (at age 21) have no upper secondary degree. The vertical axis gives the absolute number of individual school-to-work trajectories and, hence, the absolute number of young persons covered by the graph. The upper graph illustrates individual school-to-work trajectories for a total of 164,879 young Danes, the lower graph separately for a total of 61,844 young Danish non-completers. 
How to read the two graphs: Each row (line) represents an individual school-to-work trajectory, starting at age 16 and ending at age 20. At each age point, the young person is observed to be engaged in one of the five main activities identified (and described in sub-chapter 2.1). Each row thus indicates how the young person in question shifts between activities or stays in certain activities. For example, the young persons located highest up in the two graphs are in all years, from age 16 up to age 20, enrolled as full-time students, whereas the young persons situated lowest down in the two graphs show up in the dumping category of "other" (inactivity) in all these post-compulsory school years. Hence, in the upper graph covering all young Danes in our three youth cohorts, the point of departure is exactly the same as in Figure 2.1a: all young persons' main activity upon leaving compulsory school at age 16. While Figure 2.1a illustrates the average share of young Danes in the five main activity categories at each age point $(17,18,19$ and 20$)$, the upper graph in the above figure traces each young person's pathway through these activities up to age 20.

The overall impression mediated by the two graphs contained in Figure 2.3a is that the situation looks strikingly similar for all young people (upper graph) and non-completing young people (lower graph) with large shares of both groups continuing directly in post-compulsory education and moving smoothly from school into working life. Another conspicuous feature is that while many young Danes occasionally spend years outside the education system, they typically re-enter school before they turn 21.

\subsubsection{Finland: all vs. non-completers}

Figure 2.3b for Finland is constructed in the same way as the above figure for Denmark and should, as a consequence, also be interpreted in the same fashion. Hence, taken together the three Finnish youth cohorts cover a total of 193,567 young people having experienced the individual trajectories displayed in the upper graph of the figure. The lower graph, in turn, restricts these individual trajectories to those young people who still lack an upper secondary certificate when turning 21 (the noncompleters). As indicated by this lower graph, their total number in the data is 34,956 . 
Figure 2.3b: Finland: Individual school-to-work trajectories from age 16 up to age 20 for all young people (upper graph) and separately for non-completers (lower graph)
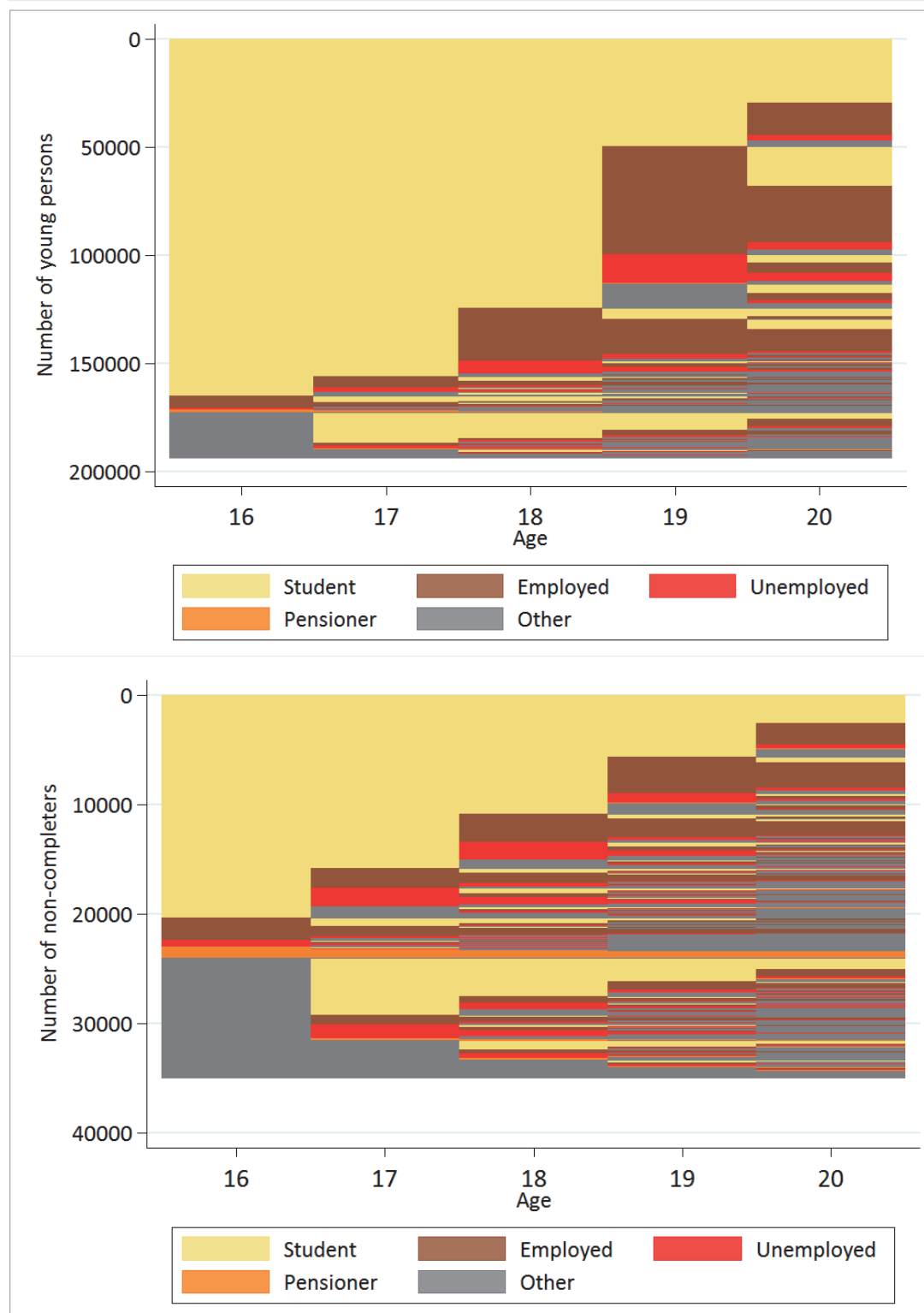

Notes: Both graphs are based on pooled information on all three youth cohorts under scrutiny. Noncompleters refer to young people who still five years after completing their compulsory education (at age 21) have no upper secondary degree. The vertical axis gives the absolute number of individual school-to-work trajectories and, hence, the absolute number of young persons covered by the graph. The upper graph illustrates individual school-to-work trajectories for a total of 193,567 young Finns, the lower graph separately for a total of 34,956 young Finnish non-completers.

How to read the two graphs: Each row (line) represents an individual school-to-work trajectory, starting at age 16 and ending at age 20. At each age point, the young person is observed to be engaged in one of the five main activities identified (and described in sub-chapter 2.1). Each row thus indicates how the young person in question shifts between activities or stays in certain activi- 
ties. For example, the young persons located highest up in the two graphs are in all years, from age 16 up to age 20, enrolled as full-time students, whereas the young persons situated lowest down in the two graphs show up in the dumping category of "other" (inactivity) in all these post-compulsory school years. Hence, in the upper graph covering all young Finns in our three youth cohorts, the point of departure is exactly the same as in Figure 2.1b: all young persons' main activity upon leaving compulsory school at age 16 . While Figure $2.1 \mathrm{~b}$ illustrates the average share of young Finns in the five main activity categories at each age point $(17,18,19$ and 20$)$, the upper graph in the above figure traces each young person's pathway through these activities up to age 20 .

The upper graph of Figure 2.3b shows that a large majority of young Finns continue in post-compulsory education, mostly directly, occasionally after a break year in other activities directly following completion of compulsory school. Post-compulsory education typically extends over a 3-year period before moving into working life. However, large numbers of young people continue in education also after this 3-year period, again either directly or after a hiatus in other activities.

The situation looks quite different for the non-completers (lower graph). While most of also non-completers continue in education after completing compulsory school, either directly or after a break year in non-education activities, their school-to-work trajectories are characterised by much more variation from the very beginning. More precisely, we see lots of young non-completers leaving post-compulsory education already after one or two years, while there are also large numbers of them staying in full-time education for three or four years or even during all years up to age 20, but without completing an upper secondary degree. When leaving post-compulsory education, they end up either in employment or in NEET activities. Noteworthy, however, is that there seems to be an pronounced difference between them depending on how many years they spent in post-compulsory education: the fewer these years, the more likely they are to end up in NEET activities. Conversely, the more years spent in post-compulsory education, despite noncompletion of an upper secondary degree, the more likely they are to move into working life. Frequent returns to school are another conspicuous feature of Finnish youth facing notable difficulties in completing an upper secondary education. The non-completers' graph also shows that there is a small group of non-completers moving straight into disability arrangements after leaving compulsory school.

\subsubsection{Norway: all vs, non-completers}

The corresponding graphs for Norway are displayed in Figure 2.3c. The upper graph highlights the individual school-to-work trajectories for all young people in the three Norwegian youth cohorts, 156,164 in total, 
while the lower graph again shows the individual school-to-work trajectories of non-completers only, 46,441 in total.

Figure 2.3c: Norway: Individual school-to-work trajectories from age 16 up to age 20 for all young people (upper graph) and separately for non-completers (lower graph)
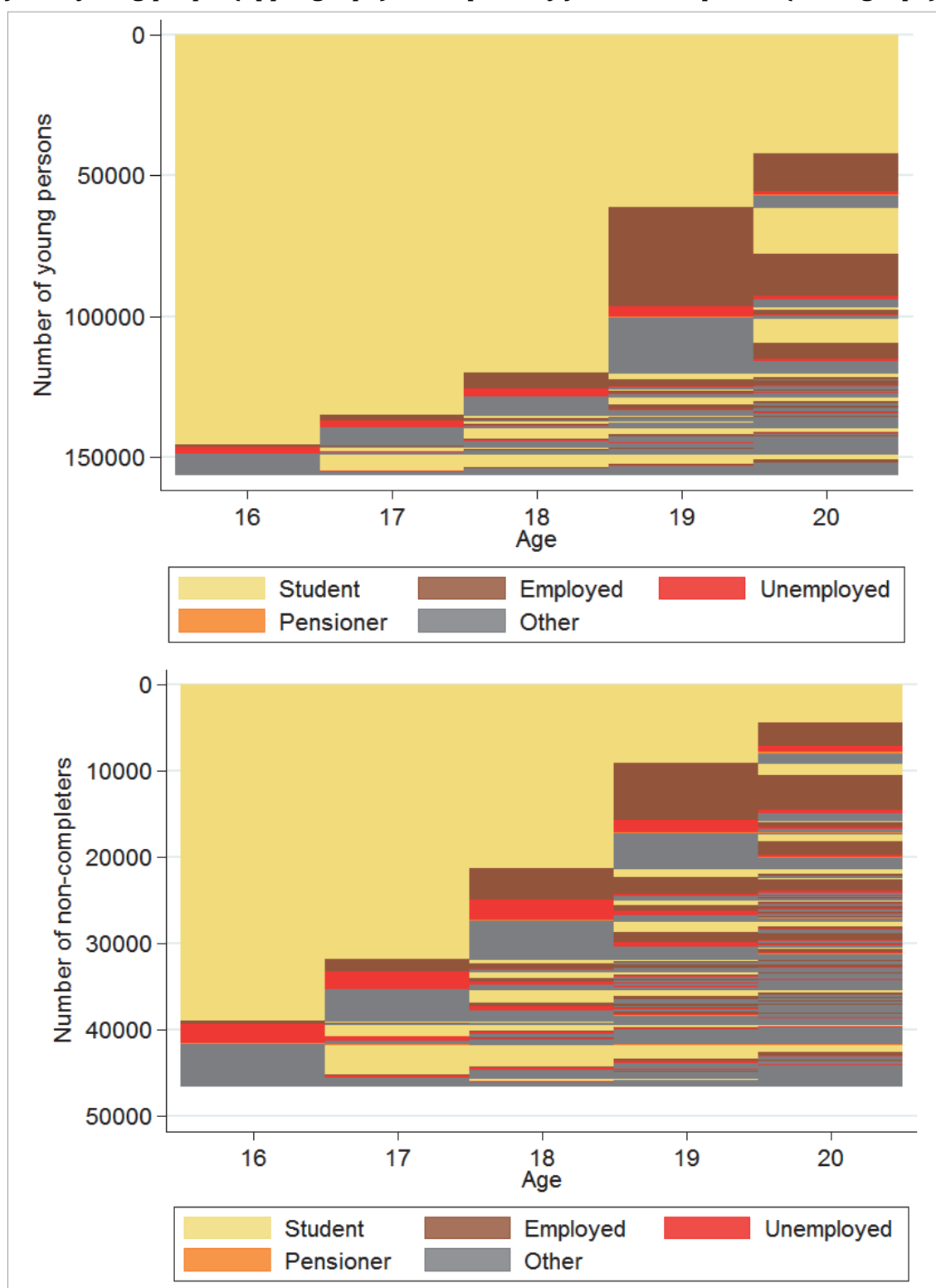

Notes: Both graphs are based on pooled information on all three youth cohorts under scrutiny. Noncompleters refer to young people who still five years after completing their compulsory education (at age 21) have no upper secondary degree. The vertical axis gives the absolute number of individual school-to-work trajectories and, hence, the absolute number of young persons covered by the graph. The upper graph illustrates individual school-to-work trajectories for a total of 156,164 young 
Norwegians, the lower graph separately for a total of 46,441 young Norwegian non-completers. How to read the two graphs: Each row (line) represents an individual school-to-work trajectory, starting at age 16 and ending at age 20. At each age point, the young person is observed to be engaged in one of the five main activities identified (and described in sub-chapter 2.1). Each row thus indicates how the young person in question shifts between activities or stays in certain activities. For example, the young persons located highest up in the two graphs are in all years, from age 16 up to age 20 , enrolled as full-time students, whereas the young persons situated lowest down in the two graphs show up in the dumping category of "other" (inactivity) in all these post-compulsory school years. Hence, in the upper graph covering all young Norwegians in our three youth cohorts, the point of departure is exactly the same as in Figure 2.1c: all young persons' main activity upon leaving compulsory school at age 16 . While Figure $2.1 \mathrm{c}$ illustrates the average share of young Norwegians in the five main activity categories at each age point $(17,18,19$ and 20$)$, the upper graph in the above figure traces each young person's pathway through these activities up to age 20 .

The individual school-to-work trajectories for the young people covered by the three Norwegian youth cohorts (upper graph) form a pattern that is highly similar to the one observed for Finland in the sense that large numbers of young people continue directly in post-compulsory education, typically stay in full-time education for three years, then move into other activities (mainly employment), but often return to school before turning 21. Likewise, and as also in Finland, many young people spend most or all of their years from age 16 to age 20 in full-time education.

On the other hand, we also observe large numbers of spells spent in inactivity (outside both education and the labour force) among Norwegian young people leaving compulsory school. However, these spells seem to be strongly concentrated to young people staying only a few years in postcompulsory education or starting post-compulsory education only after a break year. This impression is further strengthened when looking at the school-to-work trajectories of the non-completers (lower graph). Moreover, also re-entries into school among these young non-completers seem often to end in new spells spent outside both education and the labour market.

Otherwise also the situation of Norwegian non-completers resembles strongly that of Finnish non-completers: individual school-to-work trajectories forming a staircase with the probability of non-completers of moving into working life increasing, and of ending up in NEET activities decreasing with the number of years spent in post-compulsory education. Only a few years or a delayed start in post-compulsory education increase, so it seems, substantially the risk of encountering high barriers to continue in education and to move into working life already at a young age.

\subsubsection{Sweden: all vs. non-completers}

Finally we turn to the individual school-to-work trajectories obtained based on data for the three Swedish youth cohorts. The patterns formed 
by these trajectories are displayed in Figure 2.3d, again separately for all young people (upper graph) and for non-completers (lower graph).

Figure 2.3d: Sweden: Individual school-to-work trajectories from age 16 up to age 20 for all young people (upper graph) and separately for non-completers (lower graph)
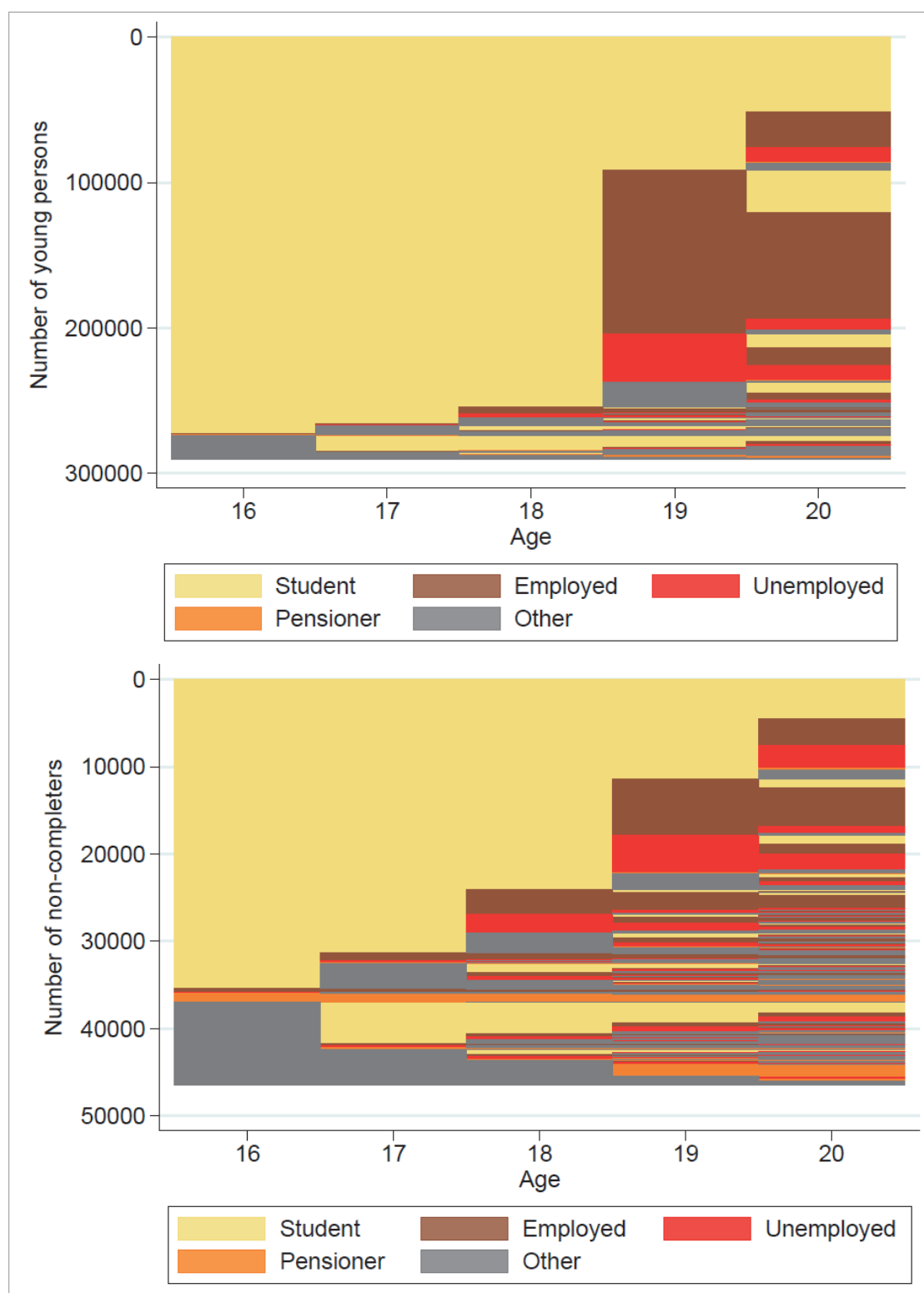

Notes: Both graphs are based on pooled information on all three youth cohorts under scrutiny. Noncompleters refer to young people who still five years after completing their compulsory education (at age 21) have no upper secondary degree. The vertical axis gives the absolute number of individual school-to-work trajectories and, hence, the absolute number of young persons covered by the graph. The upper graph illustrates individual school-to-work trajectories for a total of 290,257 young 
Swedes, the lower graph separately for a total of 46,523 young Swedish non-completers. How to read the two graphs: Each row (line) represents an individual school-to-work trajectory, starting at age 16 and ending at age 20. At each age point, the young person is observed to be engaged in one of the five main activities identified (and described in sub-chapter 2.1). Each row thus indicates how the young person in question shifts between activities or stays in certain activities. For example, the young persons located highest up in the two graphs are in all years, from age 16 up to age 20 , enrolled as full-time students, whereas the young persons situated lowest down in the two graphs show up in the dumping category of "other" (inactivity) in all these post-compulsory school years. Hence, in the upper graph covering all young Swedes in our three youth cohorts, the point of departure is exactly the same as in Figure 2.1d: all young persons' main activity upon leaving compulsory school at age 16. While Figure $2.1 \mathrm{~d}$ illustrates the average share of young Swedes in the five main activity categories at each age point $(17,18,19$ and 20$)$, the upper graph in the above figure traces each young person's pathway through these activities up to age 20 .

The dominance of full-time education is overwhelming (upper graph). Most young Swedes continue directly in post-compulsory education and stay there for three years. After this follows a year typically spent in working life, less often in NEET activities. The employment spell usually extends over several years; only occasionally does it end with the young person returning to full-time education. The activities following upon a year spent in NEET activities, after three years in upper secondary education, are more varied with prolonged inactivity being equally likely as employment or return to education.

As in both Finland and Norway, large shares of young people also spend all or most of their time up to age 20 as full-time students. The remainder of individual trajectories seems to mainly represent different combinations of spells spent interchangeably in full-time education and NEET activities. However, this impression may well be false in view of the large number of young people covered by the three Swedish youth cohorts: 290,257 in total. Accordingly, the upper graph of Figure $2.3 \mathrm{~d}$ becomes in the Swedish case extremely "compressed" with the individual trajectories so densely packed that they easily cover and, therefore, conceal each other.

Since the total number of non-completers is substantially lower $(46,523)$, the pattern of early school-to-work trajectories is much easier to assess for this group of young people from graphs like the ones in Figure 2.3d. Indeed, the impression of non-negligible shares of compulsory-school-leaving Swedish youth spending prolonged spells in NEET activities receives further support from the lower graph of Figure 2.3d. Otherwise we observe, as also for Finland and Norway, a staircase-type pattern among young Swedish non-completers with the probability of getting a job increasing, and the probability of ending up in NEET activities decreasing with the number of years spent in post-compulsory education. Another peculiarity that Sweden shares with Finland concerns 
young people receiving disability benefits. First, there is a small group of non-completers showing up as disability beneficiaries straight after leaving compulsory education. Another group of young people appears to start their post-compulsory-school trajectory outside both education and the labour force (in "other" inactivity) before eventually moving onto disability benefits.

\subsubsection{Main findings}

In this sub-chapter we have described young people's school-to-work trajectories, starting from the compulsory-school-completing age of 16 and tracing them up to age 20. Apart from presenting this kind of information for all young people in our three country-specific youth cohorts, we have illustrated the corresponding patterns separately for non-completers, that is, young people having only a basic education still at age 21 .

The graphs contained in each country-specific figure clearly reveal that there is considerable variation in individual trajectories in all four Nordic countries under study, a variation effectively concealed in the average distributions presented in sub-chapter 2.2. Simultaneously, the graphs containing all young people illustrate, once again, the impact of the countries' differently organised upper secondary education systems. In particular, in Denmark, where apprenticeships have a long tradition, the transition from school to work seems to be much smoother than in Finland, Norway and Sweden, where upper secondary education is organised in a more "school-based" manner.

The cross-country differences are not equally pronounced when it comes to the individual school-to-work trajectories of the non-completers. Indeed, in all four countries, the early school-to-work trajectories of noncompleters are found to form a "staircase" with young people leaving post-compulsory-school - some temporarily, some on a more permanent basis - at a more or less smooth pace. More precisely, for Finland, Norway and Sweden, the individual trajectories of non-completers point to a stepwisely occurring break in upper secondary education with, broadly speaking, approximately equal numbers of non-completing young people leaving after one, two or three years of full-time studies. Fewer of them are likely to leave after having been enrolled as a full-time student for more than three years. Nonetheless, they fail to complete an upper secondary degree (by age 21). Another common feature of Finnish, Norwegian and Swedish non-completers is that their probability of getting a job upon leaving post-compulsory education increases and of ending up in NEET 
activities decreases with the number of years initially spent in postcompulsory education. Hence, in all three countries, more years in upper secondary education, even without completing a degree, seem to be related to less risky school-to-work profiles up to age 20. In Chapters 7 and 8 , we return to their situation after age 20 .

The situation looks different for the Danish non-completers. In particular, young Danes turning up as non-completers by age 21 typically leave post-compulsory education already after one or two years, while they more seldom drop out after three or more years in upper secondary schooling. Moreover, also those leaving school early often return soon to full-time education or succeed in getting a job on, so it seems, a rather permanent basis. Hence, young non-completers seem to fair better in Denmark than in the other three countries. In the subsequent chapters, we will go more deeply into this matter. 


\section{School-to-work trajectories: country-specific cluster results}

In this chapter, we present a first set of results obtained by "clustering" the multitude of young people's individual school-to-work transition pathways when aged 16 to 20 , as reported in the previous chapter, by use of so-called cluster analysis. Accordingly, we start with a brief outline and discussion of the basic idea of the cluster analysis method. Only then do we turn to the results obtained for each country. It is worth stressing from the outset that these results are obtained when allowing each national dataset to form the clusters for the country in question. In other words, we do not restrict the cluster analysis in order to produce as similar clusters as possible across the four Nordic countries under study. However, the results underlying the country-specific clusters reported below are used as key inputs for the cluster analysis undertaken in the next chapters, where we explicitly aim at producing and comparing what we have labelled "common" Nordic school-to-work trajectories (Chapter 4) and "stylized" school-to-work pathways for the Nordic countries (Chapter 5).

\subsection{The cluster analysis method - a brief outline}

In the previous chapter, we showed country-specific graphs illustrating the multifaceted school-to-work transition pathways that young people follow after leaving compulsory school. Each individual trajectory paints a sequence (row) of main activities based on information on the young person's educational and labour market experiences from age 16 up to age 20. These main activities, which were described in more detail in Chapter 2, include five mutually exclusive statuses: full-time student, employed, unemployed, disability beneficiary (pensioner) and "other". As previously indicated, this mutual exclusiveness implies that the young person is for each year (age) assigned only one main activity. This exercise then produces for each young person in our data one single 
sequence of activities over the ages 16 to 20 . Based on all this information on individual school-to-work trajectories (sequences of main activities), we finally allocate our young people into a number of groups (clusters) using cluster analysis techniques.

But before describing in more detail the basic idea of the cluster analysis method, there is also reason to briefly reflect on why it is purposeful or even necessary to form such clusters. As was evident already in the previous chapter, we can trace our young people also beyond the age of 20 . For the youngest cohort (the 2003 cohort of 16-year-olds) we have information up to age 21, for the middle cohort (the 1998 cohort of 16-yearolds) up to age 26, and for the oldest cohort (the 1993 cohort of 16-yearolds) up to age 31. This information on main activities later in life will be further explored in the subsequent chapters, notably in Chapters 6 and 7, with the focus being on three specific age points, viz. age 21, 26 and 31 . The hypothesis then is that the future labour market status of young people (as indicated by their main activities) can be expected to be related not only to childhood and compulsory-school experiences but also to their educational and labour market experiences straight after completing compulsory school. In order to investigate the prevalence of such a relationship, it is necessary to cluster all young persons in a reasonable way based on their educational and labour market experiences when aged 16 to 20 , as measured by their early school-to-work trajectories. For such a clustering to make sense, the formed groups need to contain young persons who are highly similar with respect to their early educational and labour market experiences after completing compulsory school.

\subsubsection{Step 1: Sequences}

In describing the cluster analysis method, we depart from the outline of the sequence analysis method in Chapter 2 and by building on the example used in that context. In particular, we presume that three youth cohort members experience, from age 16 up to age 20, the following sequences of main activities:

- Sequence one: 11121

- Sequence two: 11211

- Sequence three: 12544 
The young person in sequence one is a full-time student (activity 1 ) at all ages up to 20, except for age 19 when the person spent most time in working life (activity 2). The situation is similar for the person representing sequence two, except that the row of full-time education years is interrupted by a spell in employment one year earlier, at age 18. The person in sequence three, in contrast, continues in post-compulsory education for one year only, moves into working life at age 17, withdraws from both education and the labour market (activity 5) at age 18, and shows up as a pensioner on disability arrangements (activity 4) at age 19 and still at age 20 .

Needless to say, the alternative trajectories (sequences of main activities) that a young person may follow are huge in number. The reason is simple: from one age to the next - e.g. from age 16 to age 17 - the young person can in principle make five different moves: stay in the same activity or move into one of the other four main activities. Accordingly, the total number of possible combinations of main activities over five ages, from 16 to 20 , is no less than $3.125(5 \times 5 \times 5 \times 5 \times 5=3.125)$.

The real-life number of alternative trajectories from age 16 up to age 20 is much smaller, though, as is also evident from the country-specific graphs presented in sub-chapter 2.4 above. For the three Danish youth cohorts under scrutiny we can identify a total of unique 813 postcompulsory-school sequences, implying that the different trajectories followed by the 164,879 young Danes belonging to these three cohorts represent about one-fourth $(813 / 3,125)$ of all possible combinations of main activities over these five age points. The number of actually observed trajectories is 1,322 for Finland, 1,071 for Norway and 767 for Sweden. Accordingly, young Danes and Swedes are more likely to follow highly similar post-compulsory-school tracks than are Finnish and Norwegian youth.

However, also these numbers of actually observed trajectories are so high that it is impossible to describe and analyse each of them separately. A major aim of the cluster analysis method is to obtain an overview of transition patterns by reducing large numbers of real-life sequences to a smaller number of classificatory categories of sequences. 


\subsubsection{Step 2: Substitutions and distances between sequences}

In our example, we have three sequences and the next step will, consequently, be to form clusters from these sequences. As described above, the educational and labour market experiences of the two persons following sequence one and sequence two are almost identical: both individuals spend most of their early post-compulsory years in full-time education, except for a temporary move into working life. The only difference between the two persons is the timing of this break before returning to education. The trajectory followed by the third person is very different: early school leaving and disability arrangements already at age 19. Hence, the early educational and labour market experiences of the persons following sequences one and two are highly different to the educational and labour market experiences of the person following sequence three. Put differently, there is a large distance from sequences one and two to sequence three, but a small distance from sequence one to sequence two. Intuitively, sequences one and two should, therefore, go into one cluster and sequence three into a separate cluster.

However, when allocating large numbers of individual trajectories into a reasonable number of clusters, we need to be able to measure the distances between sequences in an easy but reasonable way. In our example, this measure should then be small for the distance between sequences one and two, and large for the distance of these two sequences to sequence three. One way of measuring the distance between two sequences is to count the number of substitutions required for changing one sequence into the other sequence, and then use the number of substitutions needed as a measure of the distance between the two sequences. Box 3.1 illustrates this idea for our three example sequences. 


\section{Box 3.1 Calculating substitutions and distances between sequences, an example}

For measuring the distance between sequence one and sequence two below, we need to convert sequence two so that it becomes identical to sequence one.

- Sequence one: 11121

- Sequence two: 11211

- Sequence three: 12544

The two sequences differ from each other in two respects. First, the third element (activity) in sequence two contains a 2 (employed) while the third element in sequence one contains a 1 (full-time student). Hence, we need to substitute activity 2 in sequence two with activity 1 . Second, the fourth element in sequence two contains a 1 while the fourth element in sequence one contains a 2 , which requests a substitution of activity 1 in sequence two with activity 2 . These two substitutions have converted sequence two into sequence one. A similar conversion of sequence two into sequence three would demand four substitutions.

Next, the three sequences in our example are grouped into clusters according to the measured distance between them. The distance between sequence one and sequence two is two, whereas the distance from sequence three to both of these sequences is four. Sequences one and two would thus go into one cluster and sequence three into a separate cluster. This clustering reflects the intuitive notion that there is a large difference between sequences one and two, on the one hand, and sequence three, on the other hand.

It may also be noted that another way of converting one sequence into another is to delete elements and insert elements. Instead of a substitution, one element in a sequence could be deleted and another element inserted. The costs of these operations are called InDel costs (Insert and Delete). For our purposes, there is no gain in applying insertions and deletions, whereas a distinction between substitutions, deletions and insertions might be very useful in other applications (e.g. when the sequences take place over different time intervals). 
The literature uses the notion "costs" for converting one sequence into another. In the example outlined in Box 3.1, we have set the "cost" of all substitutions equal to one. However, the cost of converting one element (activity) of a sequence into another could also be set to a larger number (than one), if the difference between the two statuses is considered to be large. For example, if it is a common and natural phenomenon that young people move frequently between the statuses "student", "employed" and "unemployed", then the costs of moving between them could be seen to be low and, hence, set to one. Likewise, if a transition of young people from these activities into the status of "pensioner" or "other" is considered to be a less common and natural phenomenon, then the costs of such moves could be set higher, say, to two. Also more complex systems have been used in the literature with each move between different statutes assigned a different cost. In our analyses, we rely on the default of applying unit costs for all substitutions necessary for making sequences identical. We did experiment with different costs for different types of substitutions, but soon realised that the results from applying unit costs are more straightforward to interpret.

\subsubsection{Step 3: Forming clusters of sequences}

Above we described how to calculate the distance or similarity between sequences. In a final step, these distances are used to form clusters of sequences. In our subsequent analyses, we have applied two different procedures: clusters formed by optimal matching 2 and clusters formed by reference sequences. The main reason for applying both methods is that they have advantages as well as disadvantages when it comes to the types of analyses performed and reported in this volume. Next we give a brief description of these two clustering procedures. Some more details are provided in Box 3.2.

\footnotetext{
2 It should be noted that the term "optimal matching" is used in (at least) three senses in the literature. One sense is our application of the term as described in this sub-chapter, that is, as a method for forming clusters by an algorithm that insures that the distance between the sequences within clusters is as small as possible and that the distance between clusters is as large as possible. Another sense of the term includes all types of cluster formation on the basis of distances between sequences. In this sense of the term, cluster formation by reference sequences is a sub-category of optimal matching. A third sense of the term includes both the formation of distances between sequences and the formation of clusters into the term of optimal matching. Some studies use the term optimal matching in more than one of these three senses. The content of it is normally clear from the context, though.
} 


\section{Box 3.2 Forming clusters by optimal matching and reference sequences, an example}

\section{Optimal matching}

This standard method for forming clusters of sequences of activities means that sequences are "matched" with each other to form clusters using an "optimal" procedure in the sense that the distance is small between the sequences contained in same cluster and large between the sequences allocated into different clusters.

If forming two clusters by optimal matching based on our example with three sequences, the result will be one cluster with sequence one and sequence two and another cluster with sequence three, because the distance between sequences one and two is smaller than their distance to sequence three. If we extend the number of sequences from three to four while retaining the number of clusters (two), this additional sequence will either go into the cluster containing sequences one and two or into the cluster containing sequence three, depending on its distance to the sequences in the two clusters.

\section{Reference sequences}

The basic idea when forming clusters by use of reference sequences is that the clusters are built around predefined reference sequences.

In our example with three sequences we could pick sequence one and sequence three to be reference sequences. The last sequence, sequence two, is then to be allocated to the cluster where the reference sequence has the smallest distance to sequence two, implying that sequence two will go into the cluster with sequence one as the reference sequence. A fourth sequence would be allocated to the cluster with sequence one as the reference sequence, if the distance from this fourth sequence to sequence one is smaller than its distance to sequence three, and to the cluster with sequence three as the reference sequence, if the distance from this fourth sequence to sequence three is smaller than its distance to sequence one. This procedure is, of course, repeated when adding further sequences: the distance to sequence one and the distance to sequence three is calculated for all additional sequences and each of them is allocated either into the cluster defined by sequence one or the cluster defined by sequence three, depending on which one of the two distances is smaller. Cluster formations on the basis of more than two reference sequences are constructed in an identical way. 
Optimal matching is a relatively recent procedure ${ }^{3}$ but has, nonetheless, come to be the standard method used in the literature for forming clusters. This method forms clusters of sequences which are optimal in the sense that the distance between the sequences allocated into the same cluster is as small as possible. In other words, the sequences contained in each cluster are highly similar with the distance between them being minimised. Conversely, the distance between sequences going into different clusters is maximised, that is, as large as possible.

We use optimal matching for producing clusters separately for each of the four Nordic countries under study, and refer to these clusters as "country-specific clusters". In particular, for each country we allow the national dataset to form a total of ten clusters. While these clusters can be formed in several ways and by use of alternative procedures, we prefer to apply the common procedure in social sciences, viz. the so-called Needleman-Wunsch algorithm (see Brzinsky-Fay et al., 2006). In brief, this algorithm first divides the sequences into two clusters. Then it subdivides either one of the two clusters into two sub-clusters, giving a total of three clusters. Next, the algorithm subdivides one of these three clusters into two sub-clusters, giving a total of four clusters, and so forth. The algorithm stops when it has reached the pre-specified (by the researcher) number of clusters. The ten clusters produced for each country using national data are presented in the rest of this chapter.

The basic idea of clustering by reference sequences is that the clusters are formed around given reference sequences. In the literature, this procedure for forming clusters is, therefore, sometimes referred to as forming clusters around "ideal" sequences, "typical" sequences or "idealtypical" sequences. ${ }^{4}$ When matching by reference sequences, each sequence is allocated to the cluster where the distance of the sequence to the reference sequence is smallest. In other words, first a number of reference sequences need to be identified and only then are the other

\footnotetext{
3 The first algorithm to perform optimal matching was developed by the Russian mathematician Vladimir Levenshtein and its first scientific application was in microbiology, for the analysis of DNA sequences. Sequence analysis and optimal matching were introduced into the social sciences by the American sociologist Andrew Abbot (see Abbott, 1983; Abbott and Forrest, 1986). In a recent review of the use of optimal matching in sociology, 23 applications of the procedure were found in sociological studies (see Martin and Wiggins, 2011, p. 386)

${ }^{4}$ In contributions where the reference sequence is deducted from theoretical considerations, often of sociological origin, the term "ideal" sequence is sometimes used. Some authors first perform optimal matching, then they try to find sequences that are representative or typical for the clusters and, finally, they use these representative sequences for further analysis. In such cases, the term "typical" sequence is sometimes used, see Martin and Wiggins (2011).
} 
sequences clustered around these reference sequences according to the distance (similarity) between them. This produces a number of clusters which contains sequences that are as similar as possible to the reference sequence of the cluster. This procedure is, in effect, followed in Chapter 5 , where we start out by specifying 16 reference sequences. We then apply these same reference sequences in each of the four Nordic countries when forming clusters, that is, when allocating young people's early school-to-work trajectories across the 16 reference sequences. Hence and in contrast to the country-specific clusters reported later on in this chapter, clustering by reference sequences allows us to form clusters in the same way across all four countries: a particular sequence observed in two or more of the Nordic countries is, in each country, allocated into exactly the same reference sequence cluster. ${ }^{5}$ This also implies that we are able to compare the magnitude of distinct school-to-work trajectories across the four countries.

In this context it may also be noted that we have used one more method to form cross-country comparable clusters. In particular, for each country we have made a list of the sequences identified and the number of young people following each of these sequences. Then we have pooled this country-specific information into one big data and applied optimal matching to form clusters of sequences. Also this procedure guarantees that sequences observed in more than one country are allocated into exactly the same clusters. In this sense, the clusters formed are "common" for the four Nordic countries under study. Results obtained from using this method are reported in Chapter 4.

\footnotetext{
${ }^{5}$ We apply the Stata program to do all the analyses reported in this volume. However, Stata does not contain a module for cluster formation by reference sequences, for which reason we have written a program to perform this procedure. In many applications of cluster formations, the problem of "ties" arises, that is, a sequence has the same distance to two or more clusters. The "solution" to this problem is to allocate the sequence randomly to one of the clusters to which the sequence has the same distance. We have written our reference sequence program so that if the program randomly allocates a sequence to one particular cluster in e.g. Finland, then this sequence is randomly allocated to exactly the same cluster also in the other three Nordic countries.
} 


\subsection{Country-specific cluster results: all young people}

Next we turn to the country-specific clusters obtained when forming clusters based on the national datasets by use of optimal matching, as described above. More precisely, we use this standard clustering method to group, separately for each country, the large numbers of individual school-to-work trajectories displayed in Figures 2.1a to 2.1d of Chapter 2. Accordingly, the country-specific clusters presented below reflect the situation when pooling the information on all three youth cohorts. In other words, we use our full datasets without distinguishing between cohorts, gender or completers vs. non-completers when forming these clusters. And, as indicated above, we allocate young people's early school-to-work trajectories into a total of ten clusters, which we found - after a number of experiments - to be the best choice for our purposes, that is, for unravelling also risky transition profiles of young people leaving compulsory school.

\subsubsection{Denmark}

The pattern obtained for Denmark when allocating young Danes into ten clusters based on their early educational and labour market experiences (as measured by their sequences of main activities) is displayed in Figure 3.1 and broken down in Table 3.1. Each of the ten graphs contained in Figure 3.1 highlights the sequences of main activities that the young people allocated into that particular cluster have followed from age 16 up to age 20. As is also to be expected, each cluster is clearly dominated by certain types of school-to-work trajectories showing conspicuous similarities in crucial respects. The vertical axis of each graph gives the absolute number of young persons in the cluster. On top of each graph, there is a number in bracketed parentheses and a percentage share. The number refers to the number of the cluster given by the algorithm creating the ten clusters (according to the subdivision procedure described in the previous sub-chapter). The percentage share, in turn, shows the size of each cluster as measured by the share of young persons contained in the cluster.

The first cluster (cluster 1 ) is also the largest, containing more than three-fourths $(76.3 \%)$ of the young people in the three Danish youth cohorts under scrutiny. All of them are students at age 16, after having left compulsory school, and most of them continue in full-time education also at age 17 and still at age 18, for which reason this cluster is labelled "study track". At age 19 and 20, large shares of them are employed. Oth- 
er characteristics of the cluster are that it contains a slightly larger share of the young women (77\%) than of the young men (75.6\%), and also a slightly larger share of young people from the oldest (1993) cohort (78.1\%) than from the two younger cohorts (about 75\%). Table 3.1 also shows that the cluster covers about $88 \%$ of the young Danes having completed an upper secondary degree by age 21 , with the corresponding share being $56 \%$ for young Danes with no post-compulsory certificate still at age 21 .

Figure 3.1: Clustering of young Danes' individual school-to-work trajectories from age 16 up to age 20 into ten groups, using cluster analysis on pooled information for all three youth cohorts

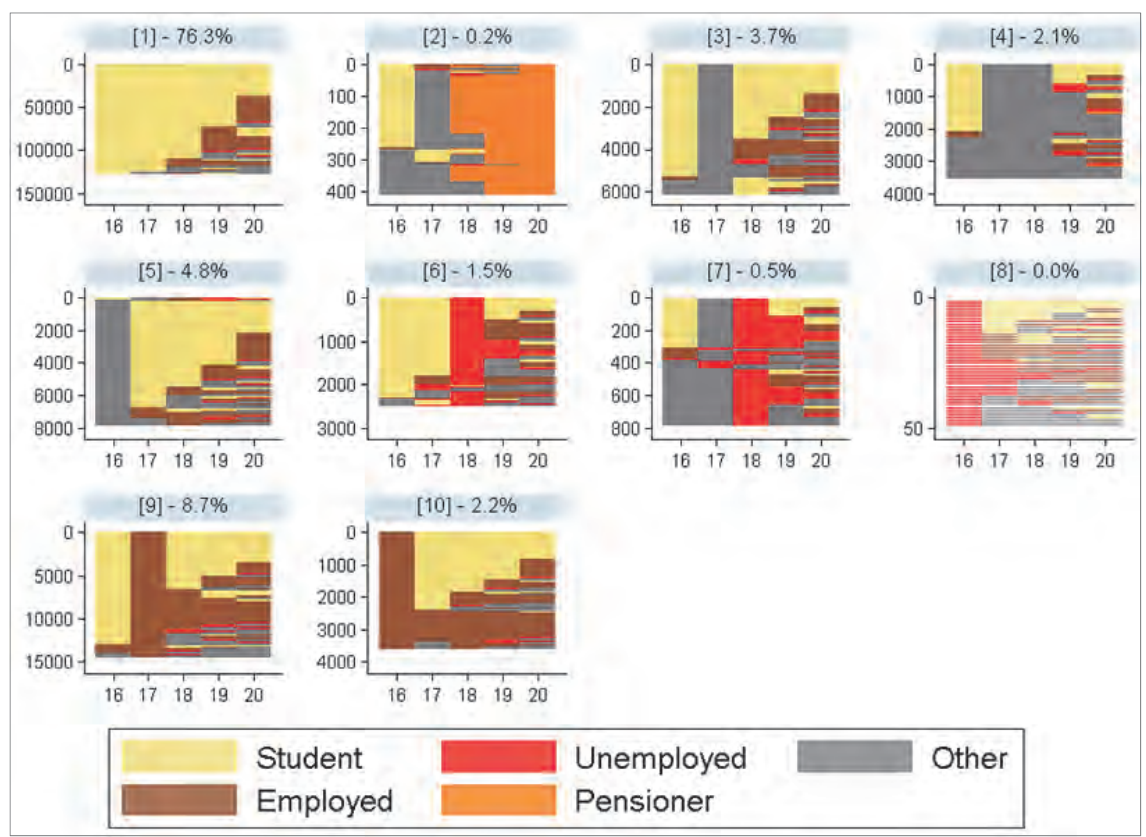

Notes: The clustering concerns individual school-to-work trajectories of young Danes when aged 16 to 20, as displayed in Figure 2.1a of Chapter 2, using the optimal matching methodology described in sub-chapter 3.1. The vertical axis of each graph gives the absolute number of young persons in the cluster, whereas the percentage share on top of each graph shows the relative share of young people in the cluster. The total number of young people is 164,879 . The number in bracketed parentheses on top of each graph refers to the number given by the algorithm creating the ten clusters by means of the subdivision procedure described sub-chapter 3.1. For more details on each cluster, see Table 3.1 below. 
Table 3.1: Distribution (\%-share) of young Danes across the ten clusters displayed in Figure 3.1, by gender, cohort and completion/non-completion of an upper secondary degree by age 21

\begin{tabular}{|c|c|c|c|c|c|c|c|}
\hline \multirow[t]{2}{*}{$\begin{array}{l}\text { Cluster: } \\
\text { number - \%-share - label }\end{array}$} & \multicolumn{2}{|c|}{ Gender } & \multicolumn{3}{|c|}{ Cohort of 16-year-olds } & \multicolumn{2}{|c|}{$\begin{array}{l}\text { Post-compulsory } \\
\text { degree by age } 21\end{array}$} \\
\hline & Male & Female & 1993 & 1998 & 2003 & Yes & No \\
\hline [1] - $76.3 \%$ study tracks & 75.6 & 77.0 & 78.1 & 75.5 & 75.2 & 88.4 & 56.0 \\
\hline$[2]-0.2 \%$ pensioner tracks & 0.3 & 0.2 & 0.0 & 0.3 & 0.4 & 0.0 & 0.7 \\
\hline [3] - $3.7 \%$ early inactivity tracks & 3.2 & 4.2 & 3.4 & 3.9 & 3.7 & 1.8 & 6.8 \\
\hline$[4]-2.1 \%$ inactivity tracks & 1.9 & 2.4 & 2.0 & 2.0 & 2.3 & 0.2 & 5.3 \\
\hline [5] - $4.8 \%$ very early inactivity tracks & 4.6 & 5.0 & 2.1 & 5.4 & 6.8 & 3.8 & 6.4 \\
\hline [6] - $1.5 \%$ study/unemployment tracks & 1.4 & 1.6 & 1.9 & 1.3 & 1.3 & 0.3 & 3.6 \\
\hline [7] - $0.5 \%$ inactivity/unempl. tracks & 0.4 & 0.5 & 0.3 & 0.5 & 0.6 & 0.0 & 1.2 \\
\hline [8] - $\quad 0.0 \%$ early unemployment tracks & 0.0 & 0.0 & 0.0 & 0.0 & 0.0 & 0.0 & 0.1 \\
\hline [9] - $8.7 \%$ early employment tracks & 9.8 & 7.6 & 10.2 & 8.8 & 7.2 & 4.0 & 16.7 \\
\hline [10] $-2.2 \%$ very early employment tracks & 2.8 & 1.5 & 1.9 & 2.2 & 2.4 & 1.5 & 3.2 \\
\hline Total & 100.0 & 100.0 & 100.0 & 100.0 & 100.0 & 100.0 & 100.0 \\
\hline$\%$-share in the pooled dataset & 51.7 & 48.3 & 34.4 & 31.0 & 34.6 & 62.6 & 37.4 \\
\hline
\end{tabular}

Notes: See Figure 3.1 above.

The second largest cluster (cluster 9) contains $8.7 \%$ of the young people in the three Danish youth cohorts. The most conspicuous feature of these young people is that all of them are in working life at age 17. Large shares of them return to education, though, while the rest of them continue in employment or move outside both education and the labour market ("other"). Cluster 10 is substantially smaller (2.2\%) but highly similar to cluster 9 in the sense that all young people contained in the cluster are employed, although already at age 16 (instead of age 17 as in cluster 9). Most young people in cluster 10 have, however, returned to education by age 17, but with a non-negligible share also continuing in working life. Table 3.1 shows that both these clusters cover a larger share of the young men than of the young women. Moreover, while the cluster 10 types of trajectories reveal a weak increase over cohorts (from 1.9\% up to $2.4 \%$ ), the share of young people following the cluster 9 types of trajectories has decreased over time (from $10.2 \%$ down to $7.2 \%$ ). Taken together, these two clusters cover slightly more than one-tenth of the three cohorts' young people, but as much as one-fifth of the young people lacking an upper secondary degree still at age 21 . It is also noteworthy that about $87 \%$ of young Danes completing compulsory school follow tracks characteristic of the three clusters discussed so far (clusters 1, 9 and 10), all of which are dominated by studying and working. 
Figure 3.1 further indicates that cluster 3 is similar to cluster 9, but with all young people being outside both education and the labour force (inactive) at age 17, instead of being employed as in cluster 9. Moreover, beyond the age of 17 , these cluster 3 youngsters do not seem to be in working life to the same extent as the cluster 9 youngsters. The setting is identical when contrasting cluster 5 to cluster 10: cluster 5 is similar to cluster 10, but with all cluster 5 youngsters being inactive at age 16, instead of being employed as in cluster 10 . Table 3.1 shows that cluster 3 and especially cluster 5 have increased in magnitude over the three cohorts. Taken together, these two clusters comprise $8.5 \%$ of the young people in the three Danish youth cohorts, with both of them covering a slightly larger share of young women than of young men. When adding this percentage share to that of clusters 1, 9 and 10, these five clusters turn out to cover almost all (95.7\%) young Danes.

Accordingly, the remaining five clusters are very small in size, comprising less than $5 \%$ of Danish youth. Clusters 6 and 7 are similar in the sense that nearly all young people contained in these two clusters are unemployed at age 18. A main difference between the two clusters, however, is that most cluster 6 youngsters studied at ages 16 and 17 while most cluster 7 youngsters were outside both education and the labour market at these ages. Despite this difference, however, a significant share of both the cluster 6 and the cluster 7 youngsters remain unemployed also after age 18 with few returning to full-time education. Instead, these youngsters face a high risk of withdrawing from the labour market, into inactivity. Taken together, these two clusters contain $2 \%$ of Danish youth. A similar share goes into cluster 4, which comprises young people who were inactive when aged 17 and 18 . Cluster 2 , in turn, contains young people who move into disability arrangements. Cluster 8, finally, contains less than $0.05 \%$ of the three cohorts' young people and is, therefore, overlooked in this context.

All in all, a main outcome of applying optimal matching to the individual school-to-work transitions of Danish youth is that an overwhelming majority falls into three specific clusters: a majority follows standard study tracks, a small group leaves the education system for employment either at age 17 or already at age 16 upon completing compulsory school, whereas a third group leaves the education system for inactivity either at age 17 or straight after compulsory school. Taken together, these clusters cover almost $96 \%$ of Danish youth. 


\subsubsection{Finland}

The ten clusters obtained from using optimal matching on the Finnish data covering all three cohorts of 16-year-olds are displayed in Figure 3.2. As for the other Nordic countries, the most conspicuous feature of these ten clusters is the overwhelming dominance of the so-called study track (cluster 1): close to $80 \%$ of compulsory-school-leaving Finnish youth go into this particular cluster, the dominant feature of which is at least three years in upper secondary school straight after completion of basic education. Moreover, as shown in Table 3.2, the share of youngsters moving early into education-dominated tracks has increased over the years, from about $75 \%$ for the 1993 cohort to $83 \%$ for the 2003 cohort. And, needless to say, most (86.5\%) young people having completed an upper secondary degree by age 21 appear in this particular cluster with the corresponding share for non-completers being substantially lower (about 42\%).

The other nine clusters are throughout of substantially smaller importance in the sense that they contain much fewer young people. Indeed, all of them have a share below 5\%, except for cluster 9 with a share of $9.5 \%$. However, taken together they cover more than onefifth of the young people belonging to the three Finnish youth cohorts under scrutiny.

About half of these clusters involve a considerable amount of studying on a full-time basis (notably clusters 2, 4, 5, 8 and 9) while the rest of them are dominated by non-study activities (clusters 3, 6, 7 and 10). Another way of characterising these nine clusters is to depart from the young person's main activity straight after completion of compulsory school. Then we see that some $3-4 \%$ continue directly in postcompulsory education but drop out after one or two years and become unemployed (cluster 2 and partly cluster 7) while a similar share drops out and start working (a majority of those in cluster 5) or withdraw from both education and the labour market (cluster 8). However, many of these young people do re-enter education before they turn 20, but far from all of them. 
Figure 3.2: Clustering of young Finns' individual school-to-work trajectories from age 16 up to age 20 into ten groups, using cluster analysis on pooled information for all three youth cohorts

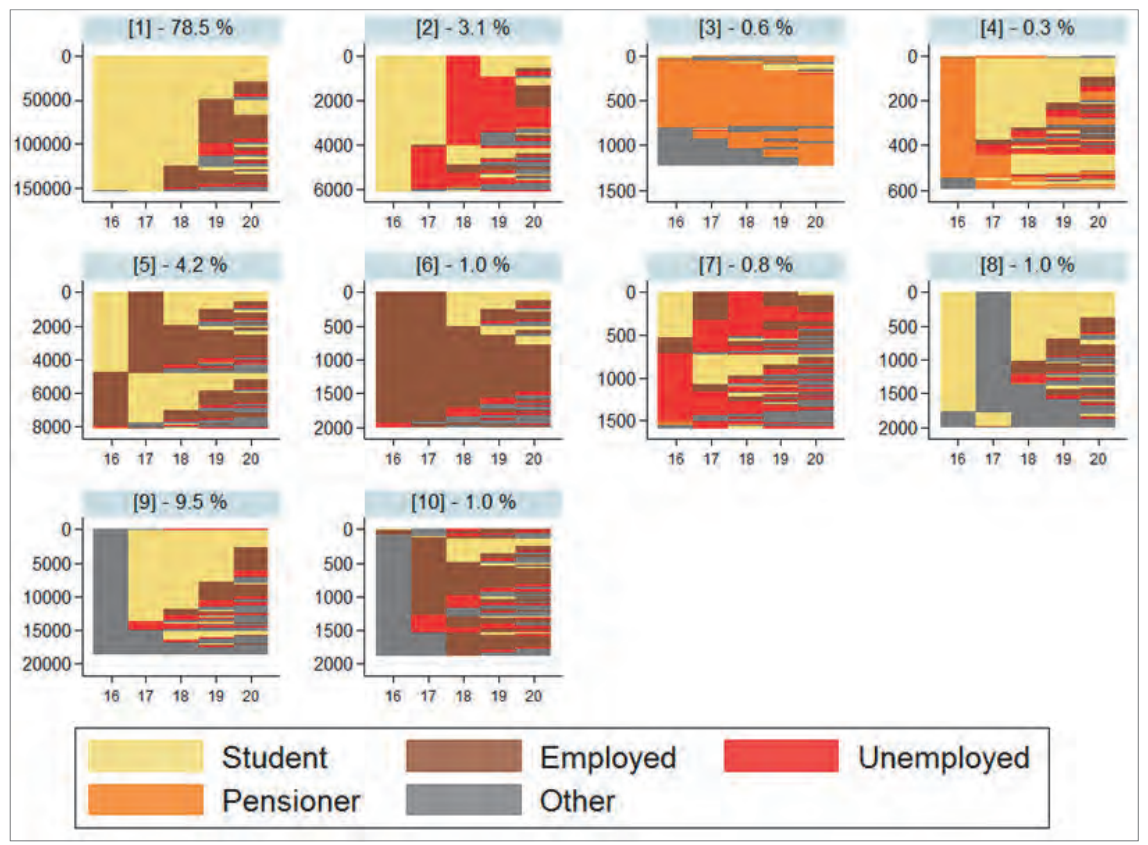

Notes: The clustering concerns individual school-to-work trajectories of young Finns when aged 16 to 20 , as displayed in Figure $2.1 \mathrm{~b}$ of Chapter 2, using the optimal matching methodology described in sub-chapter 3.1. The vertical axis of each graph gives the absolute number of young persons in the cluster, whereas the percentage share on top of each graph shows the relative share of young people in the cluster. The total number of young people is 193,567. The number in bracketed parentheses on top of each graph refers to the number given by the algorithm creating the ten clusters by means of the subdivision procedure described sub-chapter 3.1. For more details on each cluster, see Table 3.2 below.

Table 3.2: Distribution (\%-share) of young Finns across the ten clusters displayed in Figure 3.2, by gender, cohort and completion vs. non-completion of an upper secondary degree by age 21

\begin{tabular}{|c|c|c|c|c|c|c|c|}
\hline \multirow[t]{2}{*}{$\begin{array}{l}\text { Cluster: } \\
\text { number - \%-share - label }\end{array}$} & \multicolumn{2}{|c|}{ Gender } & \multicolumn{3}{|c|}{ Cohort of 16-year-olds } & \multicolumn{2}{|c|}{$\begin{array}{l}\text { Post-compulsory } \\
\text { degree by age } 21\end{array}$} \\
\hline & Male & Female & 1993 & 1998 & 2003 & Yes & No \\
\hline [1] $-78.5 \%$ study tracks & 76.7 & 80.3 & 74.9 & 77.8 & 83.0 & 86.5 & 41.9 \\
\hline [2] - $3.1 \%$ dropout/unemployment tracks & 3.6 & 2.6 & 6.5 & 2.1 & 0.6 & 2.1 & 7.6 \\
\hline [3] $-0.6 \%$ disability-benefit tracks & 0.7 & 0.6 & 0.5 & 0.7 & 0.6 & 0.0 & 3.4 \\
\hline [4] - $0.3 \%$ disability/delayed-study tracks & 0.3 & 0.3 & 0.7 & 0.2 & 0.0 & 0.2 & 0.8 \\
\hline [5] - $4.2 \%$ study/employment tracks & 4.2 & 4.1 & 5.7 & 4.1 & 2.5 & 3.5 & 7.3 \\
\hline [6] - $1.0 \%$ employment tracks & 1.2 & 0.8 & 1.7 & 0.9 & 0.4 & 0.6 & 2.8 \\
\hline [7] - $0.8 \%$ study/unemployment tracks & 0.9 & 0.8 & 1.5 & 0.6 & 0.3 & 0.3 & 3.1 \\
\hline [8] - $1.0 \%$ study-inactivity-mix tracks & 0.8 & 1.2 & 0.1 & 1.9 & 1.1 & 0.5 & 3.4 \\
\hline [9] - 9.5\% inactivity/delayed-study tracks & 10.5 & 8.5 & 7.5 & 10.5 & 10.7 & 6.1 & 25.2 \\
\hline [10] $-1.0 \%$ inactivity/employment tracks & 1.0 & 0.9 & 0.9 & 1.2 & 0.8 & 0.2 & 4.4 \\
\hline Total & 100.0 & 100.0 & 100.0 & 100.0 & 100.0 & 100.0 & 100.0 \\
\hline$\%$-share in the pooled dataset & 51.3 & 48.7 & 33.9 & 34.6 & 31.5 & 81.9 & 18.1 \\
\hline
\end{tabular}

Notes: See Figure 3.2 above. 
Still another group of young people spends the first year after compulsory school outside education: working (part of cluster 5), in unemployment (part of cluster 7), on disability benefits (cluster 4) or in other inactivity (cluster 9). In other words, many young people delay their move into post-compulsory schooling for one reason or the other. But a break year after completion of compulsory education does not always inspire the young person to re-enter school: a non-negligible share stay in noneducation activities with few, if any, schooling years breaking this track (notably clusters 3, 6 and 10).

All in all, the clustering of young Finns' individual school-to-work trajectories up to age 20 into a total of ten groups identifies basically four broad categories of young people: (1) those who continue in postcompulsory education spending most of their time up to age 20 by studying on a full-time basis; (2) those who continue in post-compulsory education and drop out after one or two years but who eventually, albeit far from always, re-enter education before turning 21; (3) those who continue in post-compulsory schooling after having spent typically one year outside education straight after compulsory school; (4) those who stay outside the education system after having completed compulsory school.

Moreover, a comparison of young men and women reveals that there are only small differences in their distributions across the ten clusters (Table 3.2). Simultaneously these shares also indicate which clusters are slightly more female or male dominated. The reason for this is simple: the Finnish data contains almost equally many young women as young men (the female share is $48.7 \%$ and the male share accordingly $51.3 \%$ ). Hence, a lower (higher) female share in Table 3.2 is also an indication of young women being slightly underrepresented (overrepresented) in that particular cluster. The clearest overrepresentation of young women occurs for cluster 8 while their clearest underrepresentation concerns clusters 2 and 6 .

When it comes to the distribution across the ten clusters of young people belonging to the three cohorts under study, Table 3.2 points to several trends. As for the male-female distribution shares, the cohort shares may be interpreted as also reflecting the relative share of each cohort in the ten clusters for the simple reason that the three cohorts are of approximately equal size, each covering about one-third of the pooled dataset (see Table 3.2). For a few clusters, we can observe an increasing share over the three cohorts implying that the youngest (2003) cohort has a more dominant role in the cluster than the oldest (1993) cohort. This holds true especially for the study track (cluster 1) but also for clusters involving early years spent outside both education and the labour market (clusters 8 and 9). Alongside there are several clusters showing a 
declining share over the three cohorts, notably clusters related to employment (clusters 5 and 6), unemployment (clusters 2 and 7) and disability benefits (cluster 4). The declining trends in relation to (registered) unemployment and disability benefits are most probably explained mainly by changes in the institutional setting, whereas the decline in employment may be seen as a logical consequence of a growing share of young people staying in full-time education. Only two clusters reveal more or less unchanged shares across the three cohorts, both of which involve minor, if any, post-compulsory studies (clusters 3 and 10).

Table 3.2 also provides information on the distribution of completers and non-completers across the ten clusters, with the non-completers again referring to those young people still lacking a post-compulsory degree by the time they turn 21 . Not surprisingly, the completers are mainly distributed across clusters representing education-dominated trajectories. However, strikingly large numbers of also non-completers seem to be heavily engaged in full-time studies, although quite a few of them show up also in clusters containing rather risky trajectories.

All in all, young Finns seem to follow quite different postcompulsory-school trajectories up to age 20. However, this clustering exercise also reveals distinct patterns characterising young Finns' early educational and labour market experiences. In particular, about nine in ten continue in education either immediately after completing compulsory school or after a year spent in non-education activities, and most of them continue in higher education or move successfully into working life. The rest of young Finns follow various employment dominated tracks after leaving compulsory school, but also high-risk tracks dominated by time spent in NEET activities.

\subsubsection{Norway}

The ten Norwegian clusters obtained from pooled information on the three youth cohorts under scrutiny are displayed in Figure 3.3. The study track (cluster 1 ) is the by far most dominant cluster also for Norway, comprising $87 \%$ of Norwegian youth and with about the same proportion (86\%) of young men and young women going into the cluster (Table 3.3). The cluster consists for the most part of young people who stay for at least three consecutive years in post-compulsory education. As is evident from Table 3.3, the study-track cluster has gained in importance over time: while $83 \%$ of the 1993 cohort of 16-year-olds followed this track, the share had grown to $89 \%$ for the 2003 cohort. Indeed, this share largely exceeds the corresponding share for Finland 
(83\%) and even more so that for Denmark (75\%) and is, in effect, very close to the share observed for Sweden (92\%). As expected, most (94\%) young people who have completed their upper secondary education by age 21 go into the study-track cluster. However, this cluster shows up as a highly dominant track also among the non-completers (young people with no upper secondary degree still by age 21 ): close to $70 \%$ of the non-completers are in this cluster. The share is strikingly high also when compared to the other Nordic countries: $58 \%$ for Sweden, $56 \%$ for Denmark and only $42 \%$ for Finland.

The remaining $13 \%$ of young Norwegians not following this study track are distributed across three broad groups of early school-to-work trajectories: study tracks with a delayed start in post-compulsory education (clusters 5, 7 and 9), study tracks with a break year from education (clusters 2 and 3) and tracks containing mainly non-study activities (clusters 4, 6, 8 and 10). As shown in Table 3.3, all these clusters are dominated by non-completers of upper secondary school, some of them overwhelmingly so.

Figure 3.3: Clustering of young Norwegians' individual school-to-work trajectories from age 16 up to age 20 into ten groups, using cluster analysis on pooled information for all three youth cohorts

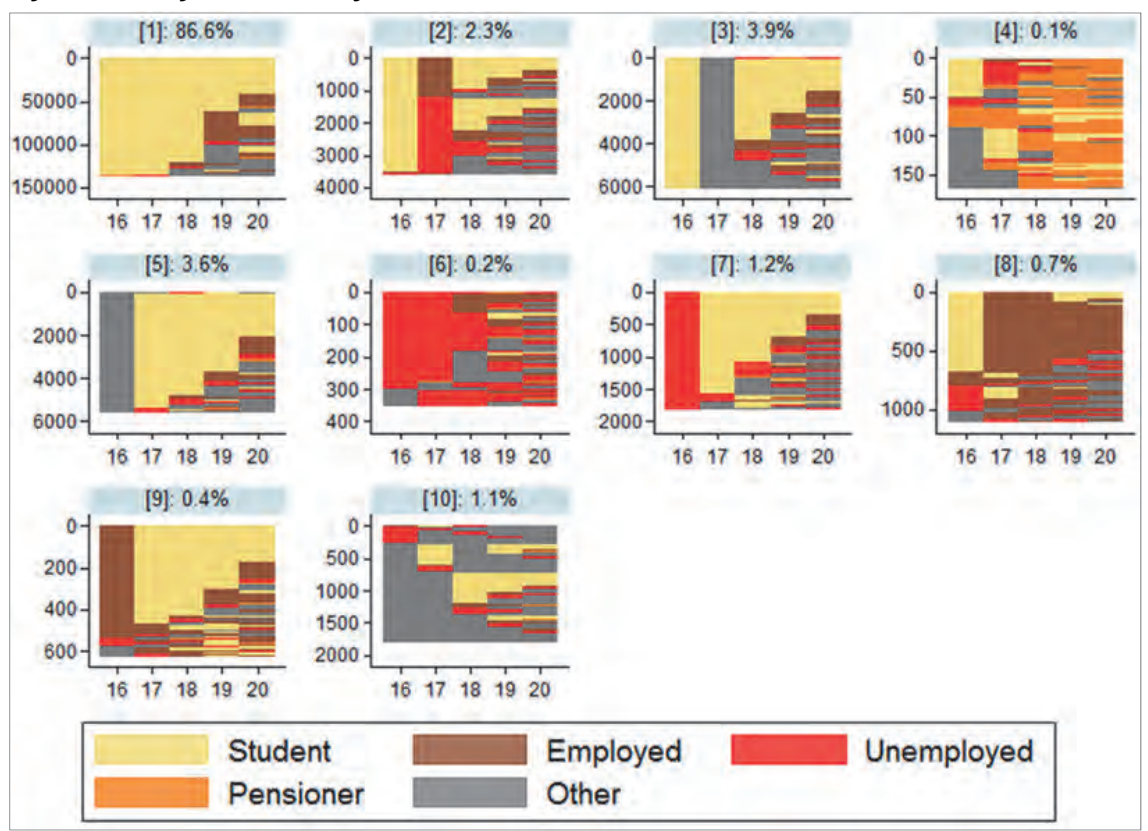

Notes: The clustering concerns individual school-to-work trajectories of young Norwegians when aged 16 to 20, as displayed in Figure 2.1c of Chapter 2, using the optimal matching methodology described in sub-chapter 3.1. The vertical axis of each graph gives the absolute number of young persons in the cluster, whereas the percentage share on top of each graph shows the relative share 
of young people in the cluster. The total number of young people is 156,164 . The number in bracketed parentheses on top of each graph refers to the number given by the algorithm creating the ten clusters by means of the subdivision procedure described sub-chapter 3.1. For more details on each cluster, see Table 3.3 below.

Table 3.3: Distribution (\%-share) of young Norwegians across the ten clusters displayed in Figure 3.3, by gender, cohort and completion/non-completion of an upper secondary degree by age 21

\begin{tabular}{|c|c|c|c|c|c|c|c|}
\hline \multirow[t]{2}{*}{$\begin{array}{l}\text { Cluster: } \\
\text { number - \%-share - label }\end{array}$} & \multicolumn{2}{|c|}{ Gender } & \multicolumn{3}{|c|}{ Cohort of 16 -year-olds } & \multicolumn{2}{|c|}{$\begin{array}{l}\text { Post-compulsory } \\
\text { degree by age } 21\end{array}$} \\
\hline & Male & Female & 1993 & 1998 & 2003 & Yes & No \\
\hline [1] - $86.6 \%$ study tracks & 86.8 & 86.3 & 82.7 & 87.6 & 89.2 & 94.2 & 68.6 \\
\hline [2] - $2.3 \%$ study/unemployment/study tracks & 2.2 & 2.3 & 2.8 & 2.1 & 1.9 & 0.7 & 6.0 \\
\hline [3] - 3.9\% study/inactivity/study tracks & 3.4 & 4.4 & 4.3 & 3.5 & 3.9 & 2.2 & 7.8 \\
\hline [4] $-0.1 \%$ disability tracks & 0.1 & 0.1 & 0.2 & 0.1 & 0.1 & 0.0 & 0.3 \\
\hline [5] - $3.6 \%$ inactivity/delayed-study tracks & 3.7 & 3.4 & 5.0 & 3.4 & 2.3 & 2.1 & 7.1 \\
\hline [6] - $0.2 \%$ unemployment tracks & 0.3 & 0.2 & 0.5 & 0.1 & 0.1 & 0.0 & 0.7 \\
\hline [7] - $1.2 \%$ unemployment/delayed-study tracks & 1.2 & 1.1 & 1.7 & 0.9 & 0.9 & 0.4 & 3.0 \\
\hline [8] - $0.7 \%$ employment tracks & 0.8 & 0.6 & 0.8 & 0.9 & 0.4 & 0.0 & 2.3 \\
\hline [9] - $0.4 \%$ employment/delayed-study tracks & 0.5 & 0.3 & 0.3 & 0.5 & 0.3 & 0.2 & 0.8 \\
\hline$[10]-1.1 \%$ inactivity tracks & 1.0 & 1.3 & 1.7 & 0.9 & 0.8 & 0.2 & 3.3 \\
\hline Total & 100.0 & 100.0 & 100.0 & 100.0 & 100.0 & 100.0 & 100.0 \\
\hline$\%$-share in the pooled dataset & 51.2 & 48.8 & 32.7 & 32.9 & 34.4 & 70.3 & 29.7 \\
\hline
\end{tabular}

Note: See Figure 3.3 above.

The break-year tracks (clusters 2 and 3) constitute the second largest group of tracks after the study track, comprising about $6 \%$ of Norwegian youth. These are young persons who start a post-compulsory education at the age of 16 but take a break year from school, filling it with nonstudy activities before re-entering education. In cluster 2 , this break year is filled with labour market activities - unemployment or employment while in cluster 3 , young people tend to spend the break year outside both education and the labour market, that is, in unknown activities. Young women are more likely than young men to follow tracks of the cluster 3 type, whereas the gender difference with respect to cluster 2 is negligible. A comparison across cohorts indicates that the share of young people following the tracks represented by cluster 2 and cluster 3 has declined over time.

A third group consists of young people who postpone their entry into post-compulsory education, so-called late starters. For the youngsters in cluster 5, the activity during this break year is unknown, that is, they cannot be found in any of the large administrative registers on which our datasets rely. This cluster comprises $3.6 \%$ of Norwegian youth, and contains a slightly larger share of young men than of young women. For $1.2 \%$ of Norwegian youth, the break year before entering upper secondary education is filled with unemployment (cluster 7). These late-starter tracks were, however, more common among young people in the oldest 
cohort (the 1993 cohort) than in the two younger cohorts (the 1998 and 2003 cohorts).

The last four tracks (clusters 4, 6, 8 and 10) comprise a very small share of Norwegian youth. A common feature of these clusters is that they contain young people who spend most of their years from age 16 up to age 20 outside the education system. In clusters 6 and 8, these youngsters are engaged in labour market activities: they spend most of their time either unemployed (cluster 6) or employed (cluster 7). Young men are marginally more likely to enter these two clusters. Clusters 4 and 10, finally, are dominated by activities outside the labour force. Most of the young people going into cluster 4 are on disability arrangements, whereas the young people allocated into cluster 10 are, when aged 16 to 20 , in activities not covered by the large administrative registers underlying our datasets, that is, their activity is not known. Also these four clusters have become less common over time.

Three observations stand out from this comparison of ten clusters. First, most young Norwegians follow a study track from age 16 up to age 20. Also those who fail to achieve an upper secondary degree by age 21, spend most of their time enrolled as full-time students. Second, there are no conspicuous gender differences in the allocation of young men and young women across these ten clusters. Although young men seem to be slightly more likely to be engaged in labour market activities at these ages, the gender difference is small. Finally, there is a clear tendency of convergence over time with an increasing share of young Norwegians following the study track (cluster 1). Conversely, it has become less common to deviate from the study track in the form of late starts and pause years. Broadly speaking, the early post-compulsory-school trajectories of young Norwegians have come to look increasingly similar.

\subsubsection{Sweden}

The clustering of young Swedes' post-compulsory-school trajectories up to age 20 results in a situation with an overwhelming majority (almost $90 \%$ ) following standard study tracks (cluster 1). More precisely, nine out of ten young Swedes start upper secondary school at age 16 and continue in full-time education without interruptions. The study track is by far the most preferred track of young people completing their upper secondary education by age 21 , at the latest: $95.4 \%$ of Swedish completers follow this track (Table 3.4.). However, substantial numbers of also non-completers - 58.5\% - show up in this cluster. An only marginally larger share of young women than of young men goes into the cluster. 
Moreover, there has been a steady increase across cohorts in the share of young Swedes following early post-compulsory-school trajectories dominated by uninterrupted studies on a full-time basis.

Apart from the dominating study-track cluster (cluster 1), two other clusters contain frequent spells of study-related activities, viz. cluster 2 and cluster 5 . While cluster 5 contains youngsters delaying their start in post-compulsory education by spending a year outside both education and the labour market, the young people contained in cluster 2 drop out - mostly temporarily - after one or two years in upper secondary education. Both clusters contain on average $3.5 \%$ of young Swedes, but with clearly fewer youngsters following these tracks in the youngest (2003) cohort as compared to the situation in the oldest (1993) cohort.

When adding the percentage shares of these two clusters to the share of cluster 1 , the outcome is that these three clusters cover as much as $96.4 \%$ of all young Swedes in the three youth cohorts under scrutiny. The same exercise for completers of an upper secondary degree by age 21 renders a percentage share that is close to $100 \%$ implying that few, if any, of them show up in the other seven clusters (Table 3.4). The corresponding percentage share is strikingly high (about $80 \%$ ) also for the non-completers, indicating that full-time education is an overwhelmingly common activity also among those young people who do not succeed in completing their upper secondary education within five years after compulsory school.

Of the remaining seven clusters, only two (clusters 8 and 10) contain about $1 \%$ of young Swedes while the rest are even smaller, each covering less than $0.5 \%$ of young Swedes. Cluster 8 represents early postcompulsory-school trajectories that mostly result in some kind of disability arrangements after prolonged spells outside both education and the labour market. Although small in magnitude, it is worth noting that the share of young people following such tracks has doubled over time: from $0.7 \%$ for the oldest cohort to $1.3 \%$ for the youngest cohort. All trajectories represented by cluster 10 start with prolonged spells in inactivity that might, so it seems, lead the young person into principally any kind of subsequent activity: education, employment or NEET activities. 
Figure 3.4: Clustering of young Swedes' individual school-to-work trajectories from age 16 up to age 20 into ten groups, using cluster analysis on pooled information for all three youth cohorts

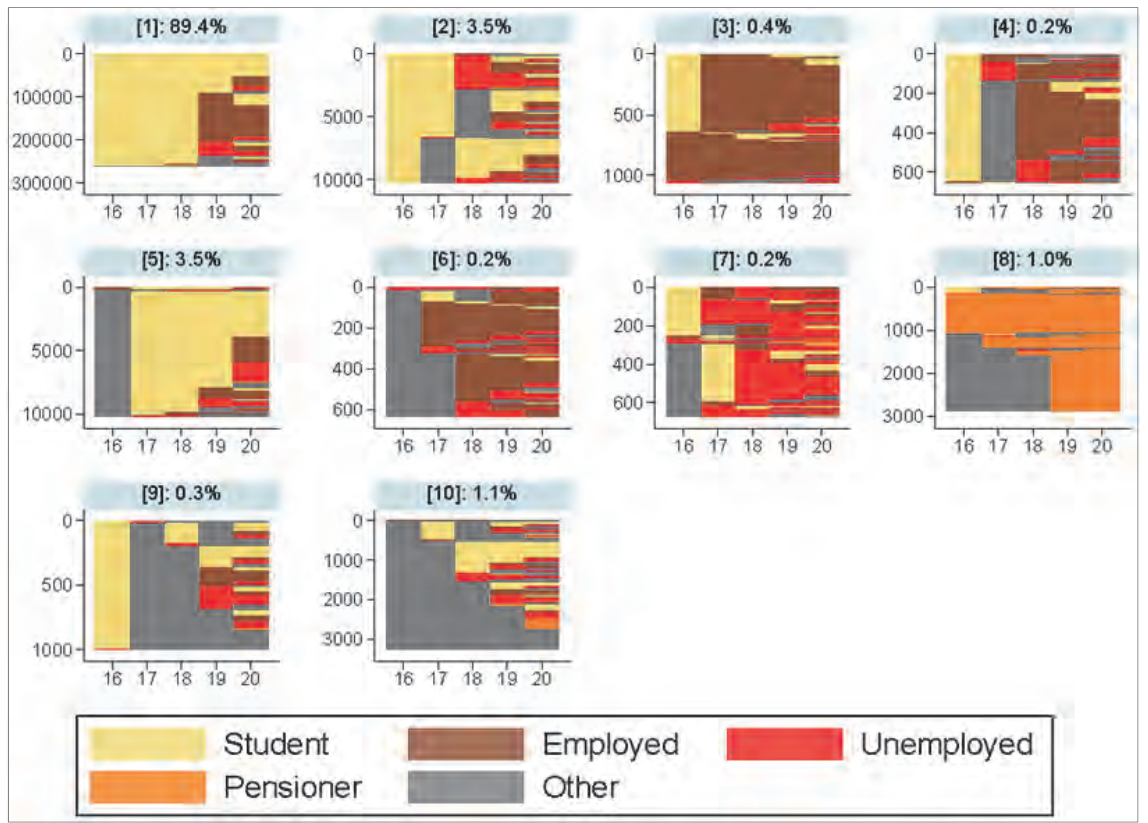

Notes: The clustering concerns individual school-to-work trajectories of young Swedes when aged 16 to 20, as displayed in Figure 2.1d of Chapter 2, using the optimal matching methodology described in sub-chapter 3.1. The vertical axis of each graph gives the absolute number of young persons in the cluster, whereas the percentage share on top of each graph shows the relative share of young people in the cluster. The total number of young people is 290,257 . The number in bracketed parentheses on top of each graph refers to the number given by the algorithm creating the ten clusters by means of the subdivision procedure described sub-chapter 3.1. For more details on each cluster, see Table 3.4 below.

All in all, the patterns emerging when allocating young Swedes' postcompulsory-school trajectories up to age 20 into ten clusters can be summarised as follows. Most young people follow three main types of tracks, all of which are heavily dominated by study activities. Adding the shares of these three clusters results in a coverage of $96.4 \%$ for all young people, close to $100 \%$ for those completing an upper secondary degree by age 21 , and about $80 \%$ for the non-completers, that is, those with only a basic education still at age 21 . Moreover, the uninterrupted study tracks (cluster 1) have gained further in importance across cohorts while there has been a corresponding decline in the more "bumpy" study tracks of the other two key clusters. The remaining seven clusters thus contain less than $4 \%$ of all young Swedes, a negligible share of completers, but about $20 \%$ of the non-completers. A common feature of these non-completer-dominated clusters is that there have been small, if any, 
changes across cohorts in the shares of young non-completers following these types of post-compulsory-school tracks. Finally it may be noted that young men and young women are distributed in a highly similar way across the ten clusters.

Table 3.4: Distribution (\%-share) of young Swedes across the ten clusters displayed in Figure 3.4, by gender, cohort and completion vs. non-completion of an upper secondary degree by age 21

\begin{tabular}{|c|c|c|c|c|c|c|c|c|}
\hline \multicolumn{2}{|c|}{$\begin{array}{l}\text { Cluster: } \\
\text { number - \%-share - label }\end{array}$} & \multicolumn{2}{|c|}{ Gender } & \multicolumn{3}{|c|}{ Cohort of 16-year olds } & \multicolumn{2}{|c|}{$\begin{array}{l}\text { Post-compulsory } \\
\text { degree by age } 21\end{array}$} \\
\hline & & Male & Female & 1993 & 1998 & 2003 & Yes & No \\
\hline [1] $-89.4 \%$ & Study tracks & 88.9 & 90.1 & 87.7 & 88.3 & 91.9 & 95.4 & 58.5 \\
\hline$[2]-3.5 \%$ & Study/pause-year/study tracks & 3.3 & 3.8 & 4.1 & 3.3 & 3.4 & 1.9 & 12.4 \\
\hline$[3]-0.4 \%$ & Dropout/employment tracks & 0.5 & 0.3 & 0.4 & 0.4 & 0.3 & 0.0 & 2.2 \\
\hline$[4]-0.2 \%$ & Dropout/inactivity/employment tracks & 0.2 & 0.3 & 0.2 & 0.3 & 0.2 & 0.0 & 1.4 \\
\hline$[5]-3.5 \%$ & Delayed-start/study tracks & 4.0 & 3.0 & 4.8 & 4.9 & 1.2 & 2.5 & 8.7 \\
\hline$[6]-0.2 \%$ & Inactivity/employment tracks & 0.2 & 0.2 & 0.2 & 0.2 & 0.2 & 0.0 & 1.3 \\
\hline$[7]-0.2 \%$ & Dropout/unemployment tracks & 0.2 & 0.2 & 0.3 & 0.2 & 0.1 & 0.0 & 1.4 \\
\hline$[8]-1.0 \%$ & Pensioner tracks & 1.1 & 0.8 & 0.7 & 0.9 & 1.3 & 0.0 & 6.1 \\
\hline$[9]-0.3 \%$ & Dropout/inactivity/mix-of-activities tracks & 0.4 & 0.3 & 0.3 & 0.4 & 0.4 & 0.1 & 1.9 \\
\hline$[10]-1.1 \%$ & Inactivity/mix-of-activities tracks & 1.3 & 1.0 & 1.2 & 1.2 & 1.0 & 0.2 & 6.1 \\
\hline \multicolumn{2}{|l|}{ Total } & 100.0 & 100.0 & 100.0 & 100.0 & 100.0 & 100.0 & 100.0 \\
\hline \multicolumn{2}{|c|}{$\%$-share in the pooled dataset } & 51.6 & 48.4 & 31.2 & 31.9 & 36.9 & 84.0 & 16.0 \\
\hline
\end{tabular}

Notes: See Figure 3.4 above.

\subsubsection{Main findings}

The clustering of young people's post-compulsory-school trajectories up to age 20 into a total of ten groups reveals interesting similarities - as well as dissimilarities - between the four countries. Indeed, despite the fact that the cluster analysis is performed separately for each country with the formation of clusters being entirely determined by the national dataset, a clear common pattern emerges across the four Nordic countries. In particular, the uninterrupted study track is by far the most prevalent cluster among Nordic youth when aged 16 to 20, with most young people in this cluster spending at least three consecutive years in education straight after completing compulsory school.

As summarised in Table 3.5, the study-track cluster contains close to $90 \%$ of Swedish youth, almost $87 \%$ of Norwegian youth, nearly $79 \%$ of Finnish youth and about $76 \%$ of Danish youth. Moreover, the share of young people going into this particular cluster has increased over cohorts, except in Denmark. Not surprisingly, a large majority of the young people having completed an upper secondary degree by age 21 show up in the study-track cluster. However, also the share of non-completers contained in the cluster is strikingly high, especially in Norway. Finally, a slightly larger share of young women than of young men is allocated into 
this cluster, with the exception of Norway where this gender difference is negligible.

This general pattern is further strengthened when adding to the study-track cluster other study-dominated clusters, that is, more nonstandard post-compulsory-school tracks followed by especially early completers of an upper secondary degree. An illustrative example of such an alternative trajectory is a delayed start in upper secondary education due to a break year after completion of compulsory school. The outcome of this exercise is shown in the lower half of Table 3.5. As is evident in the table, most completers go into these (two or three) studydominated clusters: this percentage share exceeds $90 \%$ in all four countries and is almost $100 \%$ for Sweden. In other words, the group of young completers of an upper secondary degree is remarkably homogenous in all four countries.

Conversely, few, if any, of the completers follow tracks represented by the remaining clusters. Instead, these clusters are dominated by postcompulsory-school trajectories mostly followed by youngsters lacking an upper secondary degree still at age 21 . Hence, although remarkably high shares of these young non-completers follow study-dominated tracks $-80 \%$ or more in Sweden and Norway and 66-67\% in Finland and Denmark - large shares of them follow also other types of tracks, many of which can be seen to represent high-risk transition pathways. Next we, therefore, take a closer look at non-completers' postcompulsory-school trajectories up to age 20 .

\begin{tabular}{|c|c|c|c|c|c|c|c|}
\hline & \multicolumn{2}{|c|}{ Gender } & \multicolumn{3}{|c|}{ Cohort of 16-year-olds } & \multicolumn{2}{|c|}{$\begin{array}{l}\text { Post-compulsory } \\
\text { degree by age } 21\end{array}$} \\
\hline & Male & Female & 1993 & 1998 & 2003 & Yes & No \\
\hline \multicolumn{8}{|l|}{ Study-track cluster (cluster 1) } \\
\hline Denmark - 76.3\% & 75.6 & 77.0 & 78.1 & 75.5 & 75.2 & 88.4 & 56.0 \\
\hline Finland $-78.5 \%$ & 76.7 & 80.3 & 74.9 & 77.8 & 83.0 & 86.5 & 41.9 \\
\hline Norway $-86.6 \%$ & 86.8 & 86.3 & 82.7 & 87.6 & 89.2 & 94.2 & 68.6 \\
\hline Sweden $-89.4 \%$ & 88.9 & 90.1 & 87.7 & 88.3 & 91.9 & 95.4 & 58.5 \\
\hline \multicolumn{8}{|c|}{ Combination of major study-dominated clusters } \\
\hline Denmark $-83.3 \%$ (clusters 1,5 \& 10 ) & 83.0 & 83.5 & 82.1 & 83.1 & 84.4 & 93.7 & 65.6 \\
\hline Finland $-88.0 \%$ (clusters $1 \& 9$ ) & 87.2 & 88.8 & 82.4 & 88.3 & 93.7 & 92.6 & 67.1 \\
\hline Norway $-94.1 \%$ (clusters $1,3 \& 5$ ) & 93.9 & 94.1 & 92.0 & 94.5 & 95.4 & 98.5 & 83.5 \\
\hline Sweden $-96.4 \%$ (clusters $1,2 \& 5$ ) & 96.2 & 96.9 & 96.6 & 96.5 & 96.5 & 99.8 & 79.6 \\
\hline
\end{tabular}

Note: This table is compiled based on the information presented in the country-specific tables 3.1 to 3.4 . 


\subsection{Country-specific cluster results: non-completers}

In this sub-chapter we look in more detail into the early school-to-work experiences of young people who do not succeed in achieving an upper secondary degree by the time they turn 21 . As shown in the previous sub-chapter, the non-completers distribute over groupings (clusters) of individual post-compulsory-school trajectories in a way that differs in crucial respects from completers, that is, from those young people who achieve an upper secondary certificate within five years after completion of compulsory school. Next we do not, however, depart from the ten broad clusters formed for each country in the previous sub-chapter based on each national dataset. Instead we repeat the cluster analysis on data restricted to embrace non-completers only. By excluding all completers from the data, we expect to obtain a more comprehensive picture of the post-compulsory-school pathways followed by youngsters ending up as young non-completers and, in so doing, also improve the crosscountry comparability of non-completers' early educational and labour market experiences. More precisely, we allocate the multitude on noncompleters' early school-to-work trajectories displayed in Figures 2.3a to $2.3 \mathrm{~d}$ of Chapter 2 across a total of ten clusters using optimal matching. Again, the reported results are country specific in the sense that each national dataset is allowed to unrestrictedly form these ten clusters.

\subsubsection{Denmark}

Figure 3.5 contains the ten clusters obtained for the Danish noncompleters $(61,884$ in total). The study-track cluster (cluster 1$)$ forms the largest group covering about one-half of the non-completers. This outcome is not surprising in view of the results presented in the previous sub-chapter: large shares of also non-completers spending most of their time up to age 20 in full-time education but without completing an upper secondary degree within five years after leaving compulsory school. Table 3.6, in turn, shows that a slightly larger share of young men than of young women goes into this cluster and that the size of the cluster has remained relatively stable over cohorts. 
Figure 3.5: Clustering of young Danes' individual school-to-work trajectories from age 16 up to age 20 into ten groups, using cluster analysis on pooled information for non-completers only

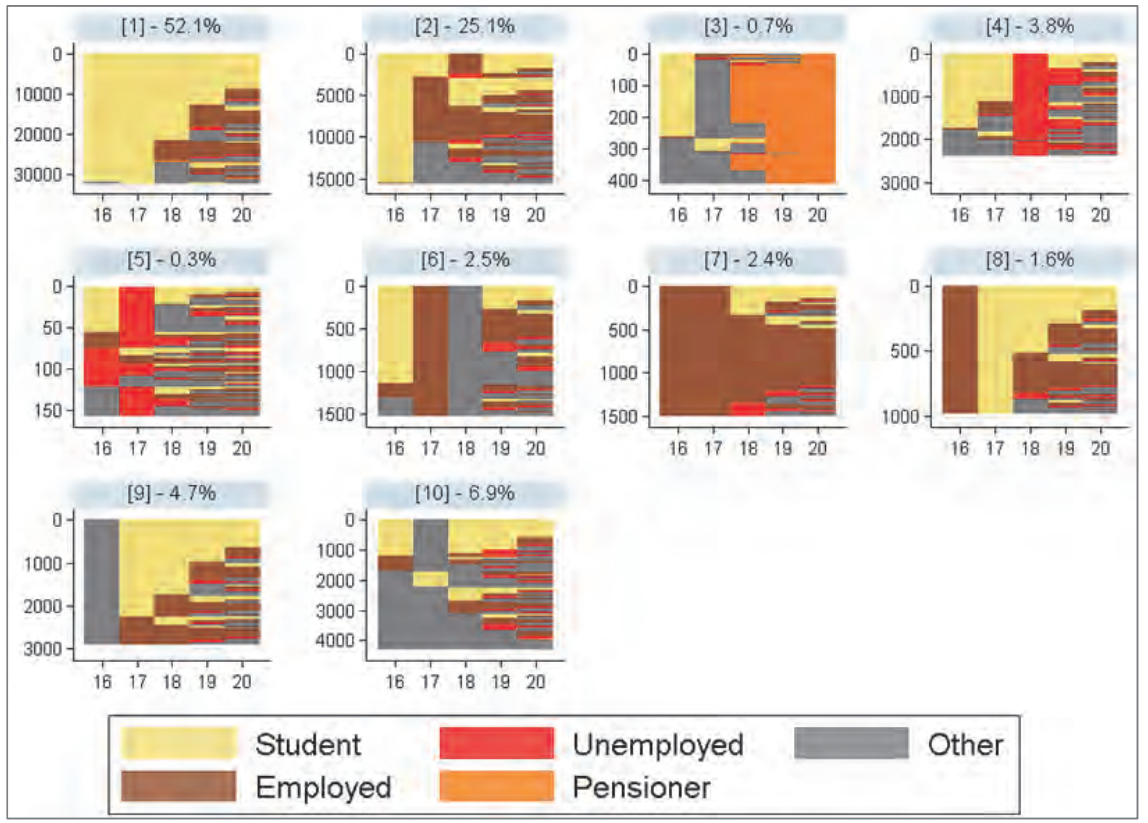

Notes: The clustering concerns individual school-to-work trajectories of Danish non-completers when aged 16 to 20, as displayed in the lower graph of Figure 2.3a in Chapter 2, using the optimal matching methodology described in sub-chapter 3.1. The vertical axis of each graph gives the absolute number of non-completers in the cluster, whereas the percentage share on top of each graph shows the relative share of non-completers in the cluster. The total number of non-completers is 61,884 . The number in bracketed parentheses on top of each graph refers to the number given by the algorithm creating the ten clusters by means of the subdivision procedure described subchapter 3.1. For more details on each cluster, see Table 3.6 below.

Other groups of young people spending much time as full-time students without obtaining an upper secondary degree by age 21 are found in cluster 8 and cluster 9 . All youngsters in cluster 8 are employed at age 16 but have re-entered the school system by age 17 , some of them temporarily and some of them on a more permanent basis. All youngsters in cluster 9 are inactive at age 16 but most of them have returned to fulltime education by age 17 . Table 3.6 shows that the difference in the male and female shares for these two clusters is marginal. Moreover, the share of non-completers contained in these two clusters has increased over time and especially so for cluster 9 , which covers $6.6 \%$ of the noncompleters in the 2003 cohort compared to only $2 \%$ in the oldest cohort. In other words, a break year before continuing in upper secondary education has become a more prevalent phenomenon among Danish noncompleters. Together with cluster 1 , these study-dominated clusters 
cover $58.4 \%$ of the non-completers, with this share being clearly higher for the 2003 cohort (60\%) than for the 1993 cohort (56\%). Hence, large shares of the non-completers spend substantial amounts of time in upper secondary education without obtaining a degree. Moreover, this trend has strengthened over time.

Table 3.6: Distribution (\%-share) of Danish non-completers across the ten clusters displayed in Figure 3.5, by gender and cohort

\begin{tabular}{|c|c|c|c|c|c|}
\hline \multirow{2}{*}{$\begin{array}{l}\text { Cluster: } \\
\text { number - \%-share - label }\end{array}$} & \multicolumn{2}{|c|}{ Gender } & \multicolumn{3}{|c|}{ Cohort of 16 -year-olds } \\
\hline & Male & Female & 1993 & 1998 & 2003 \\
\hline [1] - 52.1\% study tracks & 53.3 & 50.4 & 52.7 & 52.5 & 51.2 \\
\hline [2] - 25.1\% dropout/employment/inactivity tracks & 25.0 & 25.3 & 29.2 & 23.5 & 22.8 \\
\hline [3] - $0.7 \%$ dropout/pensioner tracks & 0.7 & 0.7 & 0.0 & 0.8 & 1.1 \\
\hline [4] - $3.8 \%$ dropout/unemployment tracks & 3.3 & 4.6 & 4.3 & 3.4 & 3.9 \\
\hline [5] - $0.3 \%$ early unemployment tracks & 0.2 & 0.4 & 0.4 & 0.3 & 0.1 \\
\hline [6] - $2.5 \%$ dropout/employment/inactivity tracks & 2.2 & 2.9 & 2.9 & 2.5 & 2.1 \\
\hline [7] - $2.4 \%$ employment tracks & 2.9 & 1.8 & 2.6 & 2.3 & 2.4 \\
\hline [8] - $1.6 \%$ early employment tracks & 1.7 & 1.4 & 1.2 & 1.7 & 1.8 \\
\hline [9] - $4.7 \%$ early inactivity tracks & 4.6 & 4.7 & 2.0 & 5.2 & 6.6 \\
\hline$[10]-6.9 \%$ inactivity tracks & 6.2 & 8.0 & 4.8 & 7.8 & 8.1 \\
\hline Total & 100.0 & 100.0 & 100.0 & 100.0 & 100.0 \\
\hline$\%$-share in the non-completers' pooled data & 58.6 & 41.4 & 32.1 & 32.4 & 35.5 \\
\hline
\end{tabular}

Notes: See Figure 3.5 above.

Two clusters contain early post-compulsory-school trajectories characterised by unemployment: cluster 4 with most non-completers having been unemployed at age 18, and cluster 5 containing non-completers having experienced unemployment at still earlier ages. A common feature of the two clusters is that this early unemployment experience often results in the non-completer continuing in unemployment or inactivity, or switching between these two NEET activities. Slightly larger shares of young women than of young men tend to follow this type of trajectory. Table 3.6 also indicates that there has been, at most, a weak decline over time in the share of non-completers following unemploymentdominated tracks after completion of compulsory school. Taken together, cluster 4 and 5 cover on average $4 \%$ of the Danish non-completers.

Cluster 7, in turn, contains non-completers who start their early postcompulsory-school career in employment. Most of them also continue in working life, so it seems, on a permanent basis. The cluster is male dominated, stable over time and covers on average $2.4 \%$ of Danish noncompleters. Employment is quite a dominating status also in cluster 2, which covers no less than $25 \%$ of the non-completers, albeit this share is clearly lower among the non-completers in the 2003 cohort (close to 23\%) than among the non-completers in the 1993 cohort (29\%). 
The early post-compulsory-school trajectories contained in cluster 6 mostly start with the non-completer dropping out from school into working life already at age 17 . However, in most cases the employment spell turns into inactivity already the following year. For some of the non-completers this withdrawal into inactivity continues, for others it is replaced by employment or full-time education. This mix-of-activities cluster is more prevalent among young women than young men. However, the share of non-completers following the quite risky trajectories contained in this cluster has declined over time, standing at $2.1 \%$ for the non-completers of cohort 2003.

Also cluster 10 contains high-risk trajectories, mostly starting with prolonged spells in inactivity straight after completion of compulsory school, with only a minor share of these youngsters returning to education. Moreover, the share of young Danes following this type of trajectory has increased substantially over time, from $4.8 \%$ in the 1993 cohort to $8.1 \%$ in the 2003 cohort. Another feature of this cluster is that it covers a clearly higher share of young women than of young men. While the early experience of non-completers appearing in cluster 3 is quite similar, these youngsters have typically become disability beneficiaries already by age 18 . Cluster 3 is marginal in size, but increasing.

All in all, Danish non-completers are found to be heavily concentrated in clusters dominated by full-time education or employment, or both. More precisely, about $52 \%$ of them follow standard study tracks. When adding the shares of non-completers following more non-standard education tracks, the outcome is that about three out of five non-completers follow education-dominated tracks, but without completing an upper secondary degree by age 21 . When further adding the shares of noncompleters in employment-dominated tracks, we end up at almost $86 \%$. Hence, a large majority of Danish non-completers follow either study- or employment-dominated tracks upon completion of compulsory school. This implies that about $14 \%$ of Danish non-completers are engaged mainly in NEET activities. The NEET share of non-completers was notably lower in the 1993 cohort than in the 2003 cohort, though.

\subsubsection{Finland}

Figure 3.6 presents the ten clusters obtained from using cluster analysis on young Finns identified as non-completers in the three cohorts of 16-year-olds under study, 35,956 in total. As already shown in Table 3.2 as well as in Table 3.5 above, a considerable share of also noncompleters spend much time as full-time students, despite the fact that 
they do not succeed in achieving an upper secondary degree within five years after completing compulsory school. Accordingly, it is hardly surprising that several of the clusters displayed in Figure 3.6 reveal conspicuous resemblance with those presented in Figure 3.2 for the full data containing both completers and non-completers.

Figure 3.6: Clustering of young Finns' individual school-to-work trajectories from age 16 up to age 20 into ten groups, using cluster analysis on pooled information for non-completers only

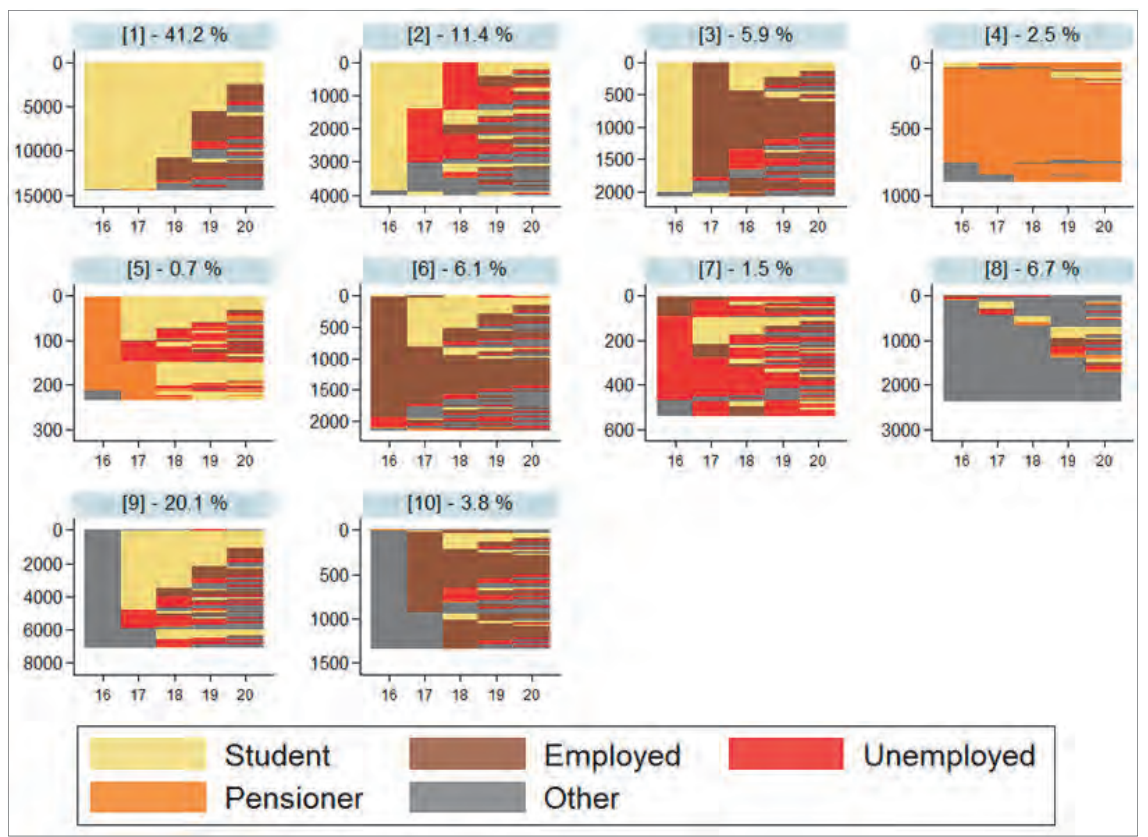

Notes: The clustering concerns individual school-to-work trajectories of Finnish non-completers when aged 16 to 20, as displayed in the lower graph of Figure 2.3b in Chapter 2, using the optimal matching methodology described in sub-chapter 3.1. The vertical axis of each graph gives the absolute number of non-completers in the cluster, whereas the percentage share on top of each graph shows the relative share of non-completers in the cluster. The total number of non-completers is 34,956 . The number in bracketed parentheses on top of each graph refers to the number given by the algorithm creating the ten clusters by means of the subdivision procedure described subchapter 3.1. For more details on each cluster, see Table 3.7 below.

As is to be expected, we identify a study-track cluster also among the non-completers, covering a substantial share of them. More precisely, two out of five non-completers $(41.2 \%)$ continue directly into postcompulsory education where they spend several consecutive years as full-time students, but without finalising an upper secondary degree. Instead, they eventually drop out, mostly to start working.

Also the next two clusters ( 2 and 3 ) resemble strongly those presented in Figure 3.2. Both start with an early dropout from post-compulsory 
education, typically already after the first year, and continue in the form of either unemployment or employment, occasionally with short reentries into the education system. Taken together, these two clusters cover about $17 \%$ of the non-completers.

Clusters 4 and 5 also have their counterparts in Figure 3.2: years as disability beneficiaries straight after completion of compulsory school, either continuing on a more or less permanent basis (cluster 4) or eventually turning into a re-entry into education (cluster 5). The noncompleters following these types of tracks are rather few in number, though (a share under 4\%).

Also the employment and unemployment tracks following straight after completion of compulsory school (clusters 6 and 7) are similar to those in Figure 3.2, as are also the inactivity-delayed tracks of postcompulsory education (cluster 9) and employment (cluster 10). Hence, the only conspicuously different cluster for the non-completers, when compared to the clusters formed for all young people in Figure 3.2, is cluster 8 . The young people going into this particular cluster spend most of their time outside both education and the labour market. Moreover, with a share of close to $7 \%$ it is the fourth largest cluster among young non-completers.

A comparison of non-completers across genders reveals considerably larger differences in cross-cluster distributions (Table 3.7) between young men and young women as compared to the situation described in Table 3.2 for the full dataset. While large shares of both male and female non-completers follow a study track (cluster 1), this track is clearly more common among young male non-completers. At the other extreme we have the inactivity-dominated track (cluster 8), which is far more common among young female non-completers. These findings are in line with what has previously been reported by Asplund and Vanhala (2014) for non-completers belonging to the youngest cohort, that is, the 2003 cohort of 16-year-olds: while there are fewer young women than young men belonging to the group of noncompleters, the female non-completers often seem to follow riskier trajectories than their male counterparts.

Finally, when it comes to the distribution across the ten clusters of non-completers from the three cohorts under study, Table 3.7 does not add much to the trends already pointed out in relation to Table 3.2. Both the study track (cluster 1 ) and the inactivity/delayed-study track (cluster 9) have gained substantially in popularity also among noncompleters. This holds true also for the inactivity track (cluster 8) which, as noted earlier, is a track distinct to non-completers only. For most of 
the other clusters we observe a declining share over cohorts, obviously mainly due to successful changes in the institutional setting. The only conspicuous exceptions from this declining trend concern the employment-dominated tracks (contained in cluster 6 and 10), the shares of which have remained approximately stable across cohorts. In other words, an early move into working life has retained its popularity among non-completers of an upper secondary degree.

Table 3.7: Distribution (\%-share) of Finnish non-completers across ten clusters as displayed in Figure 3.6, by gender and cohort

\begin{tabular}{|c|c|c|c|c|c|}
\hline \multirow{2}{*}{$\begin{array}{l}\text { Cluster: } \\
\text { number - \%-share - label }\end{array}$} & \multicolumn{2}{|c|}{ Gender } & \multicolumn{3}{|c|}{ Cohort of 16 -year-olds } \\
\hline & Male & Female & 1993 & 1998 & 2003 \\
\hline$[1]-41.2 \%$ study tracks & 42.9 & 38.8 & 35.0 & 42.1 & 46.1 \\
\hline [2] $-11.4 \%$ dropout/unemployment tracks & 10.8 & 12.2 & 19.6 & 8.7 & 6.8 \\
\hline [3] - 5.9\% dropout/employment tracks & 5.8 & 5.9 & 7.0 & 6.4 & 4.2 \\
\hline [4] $-2.5 \%$ disability-benefit (pensioner) tracks & 2.5 & 2.6 & 2.9 & 3.1 & 1.6 \\
\hline [5] $-0.7 \%$ disability/delayed-study tracks & 0.7 & 0.6 & 1.6 & 0.4 & 0.0 \\
\hline$[6]-6.1 \%$ employment tracks & 6.3 & 5.9 & 5.6 & 7.1 & 5.3 \\
\hline [7] - $1.5 \%$ unemployment tracks & 1.5 & 1.6 & 3.4 & 1.0 & 0.5 \\
\hline [8] $-6.7 \%$ inactivity tracks & 5.7 & 8.3 & 3.6 & 6.8 & 9.5 \\
\hline [9] - $20.1 \%$ inactivity/delayed-study tracks & 20.1 & 20.0 & 18.3 & 19.8 & 22.2 \\
\hline$[10]-3.8 \%$ inactivity/delayed-employment tracks & 3.5 & 4.2 & 3.0 & 4.6 & 3.7 \\
\hline Total & 100.0 & 100.0 & 100.0 & 100.0 & 100.0 \\
\hline$\%$-share in the non-completers' pooled data & 59.3 & 40.7 & 30.1 & 37.8 & 32.1 \\
\hline
\end{tabular}

Notes: See Figure 3.6 above.

On the whole, then, also Finnish non-completers show up in large numbers in post-compulsory-school tracks dominated by full-time education: about $41 \%$ of them go into the study track (cluster 1), whereas an additional $21 \%$ follow study tracks after a delayed start. Hence, more than three out of five non-completers follow education-dominated tracks, but without gaining an upper secondary certificate by age 21 . When further adding the share of non-completers following employment-dominated tracks (close to $16 \%$ ), we actually cover close to $78 \%$ of all non-completers. Hence, a substantial share of also Finnish noncompleters follow either study- or employment-dominated trajectories after having left compulsory school, a share that is about 8 percentage points lower than for Denmark. Conversely, a larger share of Finnish than of Danish non-completers show up in NEET activities: about $22 \%$ compared to $14 \%$ in Denmark. A common feature of the two countries, however, is that the share of non-completers following inactivitydominated tracks has increased over cohorts. The situation is different when it comes to unemployment: the share of Finnish non-completers going into unemployment-dominated tracks has declined remarkably 
over time. A major reason for this finding is that the school-to-work transition of the 1993 cohort started in the middle of a deep recession.

\subsubsection{Norway}

Also for Norway, the clusters formed for the non-completers look quite similar to those for the full youth population, as is evident from comparing Figure 3.7 to Figure 3.3. However, when restricting the cluster analysis to non-completers only, as in Figure 3.7, we see a more even distribution of young people across the different tracks. Again, the dominant cluster is the standard study track (cluster 1), which contains almost $70 \%$ of the non-completers. Young men are more likely than young women to pursue a study track without completing an upper secondary degree by age 21 . Moreover, the time trend towards convergence of tracks is even stronger for the non-completers than for the full youth population: while the study track covers close to $59 \%$ of the noncompleters in the 1993 cohort, the corresponding share for noncompleters in the 1998 and 2003 cohorts is 71 and $77 \%$, respectively. In other words, the phenomenon of following standard study tracks without completion of an upper secondary degree by age 21 has become increasingly common among young Norwegians.

The other nine clusters, covering almost one-third of the noncompleters, may be divided into three broad groups: study tracks with a delayed start in post-compulsory education (clusters 6 and 10), early dropout from upper secondary education (clusters 3, 4 and 7) and tracks mainly dominated by non-study activities (clusters 2, 5, 8 and 9). Earlydropout tracks are followed by a relatively large share of young Norwegians: close to $15 \%$ of the non-completers show up in this type of track. Another large group consists of late starters with delayed entry into post-compulsory education (11\%), while the non-study tracks cover only $6 \%$ of the non-completers. 
Figure 3.7: Clustering of young Norwegians' individual school-to-work trajectories from age 16 up to age 20 into ten groups, using cluster analysis on pooled information for non-completers only

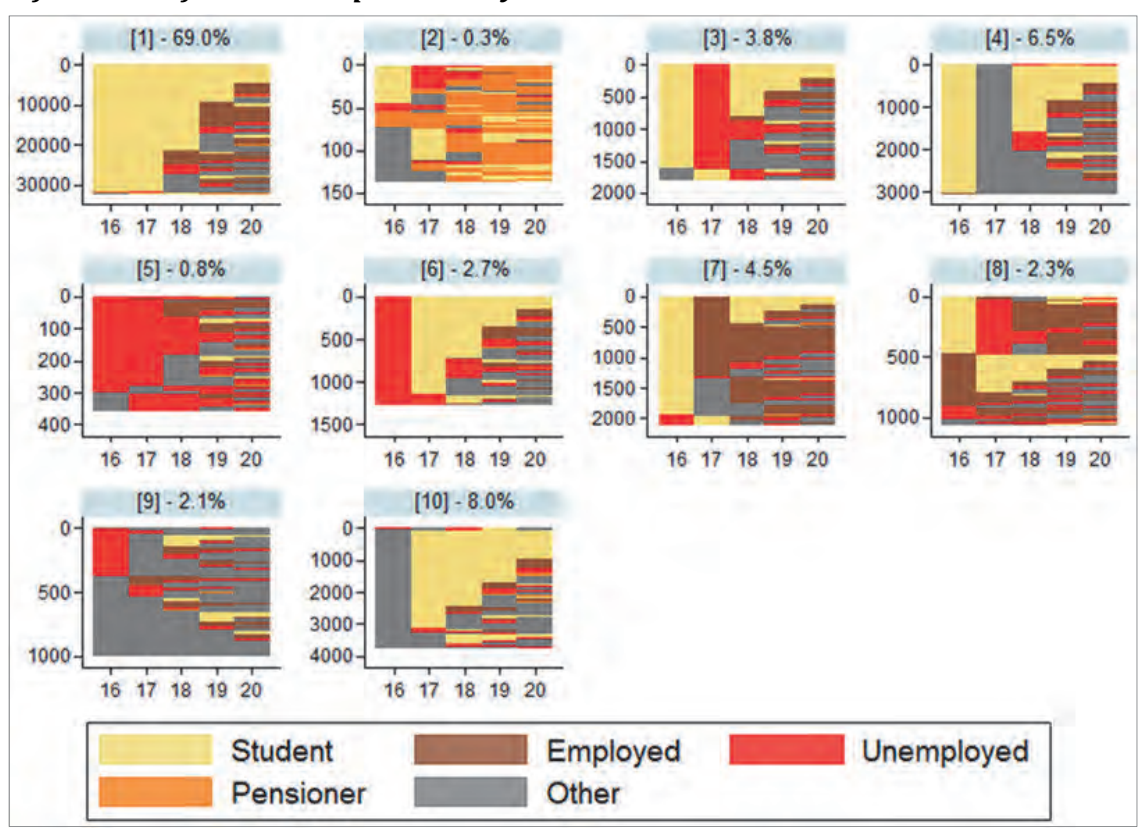

Notes: The clustering concerns individual school-to-work trajectories of Norwegian non-completers when aged 16 to 20, as displayed in the lower graph of Figure 2.3c in Chapter 2, using the optimal matching methodology described in sub-chapter 3.1. The vertical axis of each graph gives the absolute number of non-completers in the cluster, whereas the percentage share on top of each graph shows the relative share of non-completers in the cluster. The total number of non-completers is 46,441 . The number in bracketed parentheses on top of each graph refers to the number given by the algorithm creating the ten clusters by means of the subdivision procedure described subchapter 3.1. For more details on each cluster, see Table 3.8 below.

The early-dropout clusters differ from each other with respect to the activity dominating after school dropout: almost $4 \%$ become unemployed (cluster 3) and close to 5\% move into working life (cluster 7), whereas close to $7 \%$ withdraw from both education and the labour market, that is, disappear from all large administrative registers (cluster 4). Notable shares of these early school-dropouts return to education, but far from all. Moreover, these early-school-dropout tracks are typically more common among young women than among young men. This holds true especially for the early-dropout/inactivity tracks (cluster 4). Although the time trend across cohorts for these three early-dropout tracks is not equally clear-cut as for the standard study tracks, a comparison between non-completers in the oldest (1993) cohort and non-completers in the youngest (2003) cohort indicates that the probability of young Norwegians following these types of early-dropout tracks has declined over time (Table 3.8). 
Table 3.8: Distribution (\%-share) of Norwegians non-completers across ten clusters as displayed in Figure 3.7, by gender and cohort

\begin{tabular}{|c|c|c|c|c|c|c|}
\hline \multirow{2}{*}{\multicolumn{2}{|c|}{$\begin{array}{l}\text { Cluster: } \\
\text { number - \%-share - label }\end{array}$}} & \multicolumn{2}{|c|}{ Gender } & \multicolumn{3}{|c|}{ Cohort of 16-year-olds } \\
\hline & & \multirow{2}{*}{$\begin{array}{r}\text { Male } \\
70.9\end{array}$} & \multirow{2}{*}{$\begin{array}{r}\text { Female } \\
66.3\end{array}$} & \multirow{2}{*}{$\begin{array}{r}1993 \\
58.6\end{array}$} & \multirow{2}{*}{$\begin{array}{r}1998 \\
70.6\end{array}$} & \multirow{2}{*}{$\begin{array}{r}2003 \\
76.5\end{array}$} \\
\hline [1] & $69.0 \%$ study tracks & & & & & \\
\hline [2] & $0.3 \%$ disability-benefit tracks & 0.3 & 0.3 & 0.4 & 0.2 & 0.3 \\
\hline [3] & 3.8\% early-dropout/unemployment tracks & 3.5 & 4.2 & 5.3 & 3.1 & 3.2 \\
\hline [4] & $6.5 \%$ early-dropout/inactivity tracks & 5.6 & 7.8 & 7.7 & 5.9 & 6.0 \\
\hline [5] & $0.8 \%$ unemployment tracks & 0.7 & 0.8 & 1.6 & 0.4 & 0.4 \\
\hline [6] & $2.7 \%$ unemployment/delayed-study tracks & 2.6 & 2.9 & 3.9 & 2.1 & 2.2 \\
\hline [7] & $4.5 \%$ early-dropout/employment tracks & 4.6 & 4.5 & 4.9 & 5.7 & 3.1 \\
\hline [8] & $2.3 \%$ employment/unemployment tracks & 2.4 & 2.2 & 2.8 & 2.7 & 1.4 \\
\hline & $2.1 \%$ inactivity tracks & 1.6 & 2.9 & 3.6 & 1.8 & 1.2 \\
\hline [10] & $8.0 \%$ inactivity/delayed-study tracks & 7.8 & 8.2 & 11.2 & 7.5 & 5.7 \\
\hline \multicolumn{2}{|c|}{ Total } & 100.0 & 100.0 & 100.0 & 100.0 & 100.0 \\
\hline \multicolumn{2}{|r|}{$\%$-share in the non-completers' pooled dataset } & 58.0 & 42.0 & 31.4 & 32.2 & 36.4 \\
\hline
\end{tabular}

Notes: See Figure 3.7 above.

Clusters 6 and 10 represent delayed-study tracks. These two clusters contain young people who take a break year before continuing in postcompulsory education. Cluster 10 is, in effect, the second largest cluster after the study-track cluster, comprising $8 \%$ of the non-completers. Young people delaying their start in post-compulsory education typically spend their break year outside both education and the labour market. However, in contrast to the situation in both Denmark and Finland, the share of young Norwegians following such tracks has declined markedly over time (Table 3.8). The other delayed-study-track cluster, which is notably smaller in size $(2.7 \%)$, includes youngsters who spend a year mainly in unemployment - mostly in some form of active labour market policy (ALMP) program - before continuing in post-compulsory education. Also the share of non-completers following these types of tracks has declined across cohorts, though. Larger shares of young women than of young men tend to follow delayed-study tracks.

The four remaining clusters (2, 5, 8 and 9) contain young people spending most of their time pursuing non-study activities after completing compulsory school. In clusters 5 and 8 , these young people are mostly either employed or unemployed: around $2 \%$ of the non-completers spend their post-compulsory-school years up to age 20 by switching between employment and unemployment (cluster 8) while a minor share of them $(0.8 \%)$ is for the most part unemployed (cluster 5 ). About $2 \%$ of the non-completers follow tracks dominated by time spent outside both education and the labour market, in unknown inactivity (cluster 8$)$, and a very small share of them $(0.3 \%)$ is receiving health-related benefits (cluster 2). All these clusters were more prevalent in the oldest (1993) cohort. 
The overall picture emerging for young Norwegians having achieved no post-compulsory degree by age 21 is quite clear-cut. In particular, we can identify two parallel trends over cohorts. First, there has been a strong tendency of convergence towards standard study tracks also among the non-completers, whereas a declining share of them follows other types of post-compulsory-school tracks, many of which involve high-risk elements. Put differently, a growing share of also noncompleters continue directly in post-compulsory education and spend most or all of their time up to age 20 as full-time students, but without finishing an upper secondary degree within five years after completing compulsory school. Second, a growing share of Norwegian youth is facing this situation: the share of non-completers has been on the rise, from $28.5 \%$ in the oldest (1993) cohort to $31.5 \%$ in the youngest (2003) cohort, a trend that became evident already in Chapter 2 .

\subsubsection{Sweden}

More than two-thirds (69.4\%) of the Swedish non-completers follow standard-type study tracks (cluster 1), implying that they continue directly in post-compulsory education for a number of years but fail to complete an upper secondary degree by age 21 . This is a remarkably high share. Only for Norway do we observe a share of a similar size (69\%) while it is substantially lower for Danish and Finnish noncompleters (58 and 41\%, respectively). As also in the other Nordic countries, young men are more likely to continue studying on a full-time basis without completing an upper secondary degree within five years after completing compulsory school, whereas the situation is the opposite among young women, whose full-time study tracks more often result in an upper secondary certificate by age 21 , at the latest. Additionally, we see a clear time trend in the share of non-completers engaged in fulltime studies: $67 \%$ for the non-completers in the oldest (1993) cohort compared to $72 \%$ of the non-completers in the youngest (2003) cohort (Table 3.9). Hence, larger shares of Swedish non-completers spend increasingly more time in post-compulsory education, but without completing an upper secondary degree by age 21 . Moreover, as shown in Chapter 2, few of these non-completers finalise an upper secondary degree after age 21 . 
Figure 3.8: Clustering of young Swedes' individual school-to-work trajectories from age 16 up to age 20 into ten groups, using cluster analysis on pooled information for non-completers only

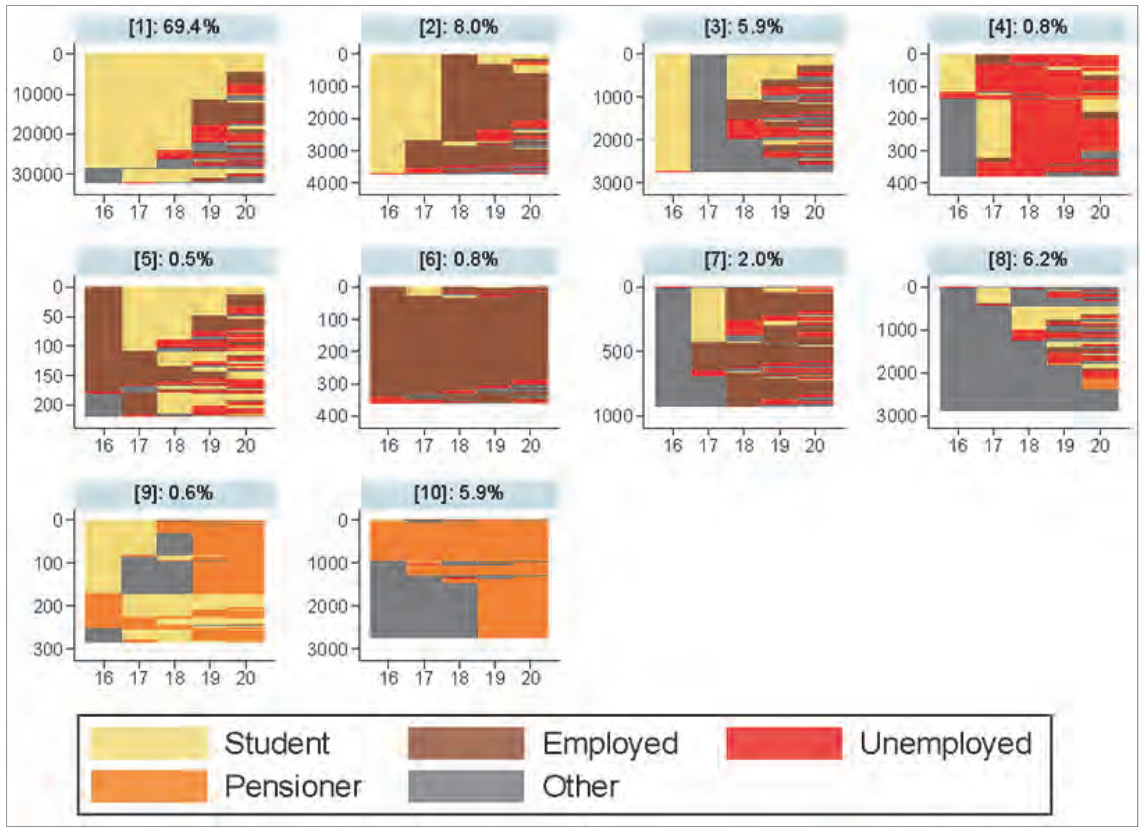

Notes: The clustering concerns individual school-to-work trajectories of Swedish non-completers when aged 16 to 20, as displayed in the lower graph of Figure 2.3d in Chapter 2, using the optimal matching methodology described in sub-chapter 3.1. The vertical axis of each graph gives the absolute number of non-completers in the cluster, whereas the percentage share on top of each graph shows the relative share of non-completers in the cluster. The total number of non-completers is 46,523 . The number in bracketed parentheses on top of each graph refers to the number given by the algorithm creating the ten clusters by means of the subdivision procedure described subchapter 3.1. For more details on each cluster, see Table 3.9 below.

Table 3.9: Distribution (\%-share) of Swedish non-completers across ten clusters as displayed in Figure 3.8 , by gender and cohort

\begin{tabular}{|c|c|c|c|c|c|}
\hline \multirow{2}{*}{$\begin{array}{l}\text { Cluster: } \\
\text { number - \%-share - label }\end{array}$} & \multicolumn{2}{|c|}{ Gender } & \multicolumn{3}{|c|}{ Cohort of 16 -year-olds } \\
\hline & Male & Female & 1993 & 1998 & 2003 \\
\hline [1] - $69.4 \%$ study tracks & 70.1 & 68.5 & 67.0 & 68.8 & 71.8 \\
\hline [2] - $8.0 \%$ dropout/employment tracks & 7.0 & 9.2 & 8.0 & 10.1 & 6.1 \\
\hline [3] - $5.9 \%$ dropout/mix-of-activities tracks & 5.4 & 6.5 & 7.1 & 5.5 & 5.3 \\
\hline [4] - $0.8 \%$ dropout/unemployment tracks & 0.8 & 0.8 & 1.4 & 0.7 & 0.5 \\
\hline [5] - $0.5 \%$ employment-delayed-start tracks & 0.3 & 0.7 & 0.8 & 0.4 & 0.3 \\
\hline [6] - $0.8 \%$ employment tracks & 1.0 & 0.5 & 1.0 & 0.8 & 0.7 \\
\hline [7] - $2.0 \%$ inactivity-delayed-employment tracks & 1.9 & 2.0 & 2.4 & 2.3 & 1.4 \\
\hline$[8]-6.2 \%$ inactivity tracks & 7.6 & 5.7 & 7.1 & 6.0 & 5.7 \\
\hline [9] - $0.6 \%$ dropout/pensioner tracks & 0.6 & 0.6 & 0.5 & 0.6 & 0.7 \\
\hline [10] - $5.9 \%$ inactivity/pensioner tracks & 6.2 & 5.5 & 4.7 & 4.9 & 7.7 \\
\hline Total & 100.0 & 100.0 & 100.0 & 100.0 & 100.0 \\
\hline$\%$-share in the non-completers' pooled dataset & 56.5 & 43.5 & 28.2 & 33.8 & 38.0 \\
\hline
\end{tabular}

Notes: See Figure 3.8 above. 
The distribution of the remaining one-third of non-completers across the other nine clusters looks quite uneven in the sense that some clusters embrace relatively large shares of non-completers while the size of other clusters is minor. The largest group consists of clusters characterised by school-dropout tracks, notably clusters 2,3 and 4 . In total, close to $15 \%$ of Swedish non-completers follow this type of track upon leaving compulsory school. However, the main activity after the young person's dropout from school differs considerably between the three clusters. More precisely, cluster 2 with a share of $8 \%$ of the non-completers contains school-dropouts who move into working life and also succeed in staying employed. Only occasionally does the working spell turn into unemployment or inactivity, or end with the young person returning to school. In cluster 3 with a share of $6 \%$, the dropout occurs already after one year in post-compulsory school and results in the young person staying outside both education and the labour market for typically one year. A majority of them continue in non-study activities, either directly or after a temporary return to education, with NEET activities being more common than employment. In cluster 4, finally, school dropout is mostly followed by prolonged spells in unemployment. This cluster, however, comprises less than $1 \%$ of the non-completers. Larger shares of young women than of young men follow the dropout tracks of clusters 2 and 3, whereas the gender shares are identical for cluster 4 (Table 3.8). A common feature of all three dropout clusters is that their importance has declined over time, especially when comparing the youngest (2003) cohort to the oldest (1993) cohort.

Another relatively large group of non-completers follow postcompulsory tracks starting from non-study activities, with the young person then moving either into working life (clusters 5 and 6) or withdrawing from both education and the labour market (clusters 7 and 8). Otherwise, these four clusters differ quite substantially from each other. Cluster 5 , which is minor in size covering only $0.5 \%$ of Swedish noncompleters, contains tracks where initial employment turns into reentry into the education system, albeit often also into unemployment. In cluster 6 , which is only marginally larger in size $(0.8 \%)$, initial employment seems to be more permanent in nature with most of the noncompleters in this cluster being employed during all years from age 16 up to age 20 . Also the tracks contained in cluster 7 , covering $2 \%$ of Swedish non-completers, tend to turn into prolonged employment spells but only after one or two early years spent in inactivity. However, compared to the employment tracks of the non-completers in cluster 6 , the employment tracks of cluster 7 tend to involve more breaks, notably in the 
form of unemployment. Cluster 8, finally, covers more than $6 \%$ of the non-completers and contains tracks starting with prolonged spells outside both education and the labour market. The longer these spells, the more likely they turn into NEET activities. Returns to school occur only occasionally and mostly for short periods. However, also these types of tracks have become less common among the non-completers. When it comes to the distribution of young men and young women across the four clusters, there is no clear pattern discernible: some of the tracks are more typical among young women while others are more typical among young men.

The last two clusters ( 9 and 10) are closely related to disability arrangements. Taken together, they cover $6.5 \%$ of Swedish noncompleters. However, cluster 9 is small in size $(0.6 \%)$, covers equal shares of young men and women, and has increased only marginally in size across cohorts. Most young people covered by disability arrangements follow the tracks contained in cluster 10: disability benefits straight after compulsory school or after a post-compulsory-school period of mostly three years spent in inactivity, that is, outside both education and the labour market.

Broadly speaking, the Swedish situation of non-completers may be summarised as follows. Remarkably large numbers (close to 70\%) continue directly in post-compulsory education and typically stay there for three years, often longer. A distinct feature of Swedish non-completers, however, is that few of them move into such study tracks after a break year after completion of compulsory school. Also employmentdominated early-age tracks are quite common among Swedish noncompleters. When adding the shares of non-completers following typical employment tracks to the shares of those in standard study tracks, we end up covering almost $81 \%$ of all non-completers. This implies that close to $20 \%$ of Swedish non-completers go at an early age into NEETdominated tracks, with the role of pure unemployment tracks being marginal. Instead, they spend most of their years up to age 20, at least, outside both education and the labour market, in unknown inactivity.

\subsubsection{Main findings}

This country-specific analysis of non-completers, that is, young people with no upper secondary degree still at age 21 , reveals that Nordic noncompleters have much in common, although they also differ in certain crucial respects. Next, we try to summarise these similarities and dissimilarities by means of Table 3.10. 
Strikingly large shares of non-completers in Norway and Sweden follow standard study tracks straight after completing compulsory school. However, when adding study tracks characterised by a delayed start in post-compulsory education, Norway outperforms Sweden, while Denmark and Finland move closer to Sweden. As can be concluded from Table 3.10, we identify small shares of Swedish non-completers delaying their start in post-compulsory education, whereas this is quite common in the other three Nordic countries under study and especially in Finland. The time trend across cohorts implies, in turn, that increasingly larger shares of Nordic non-completers follow study-dominated tracks but without achieving an upper secondary degree within five years after leaving compulsory school. Another common feature of the four Nordic countries is that larger shares of young men than of young women follow study tracks without completing an upper secondary degree by age 21. As indicated earlier, the opposite holds true for completers. Hence, full-time education without graduation by age 21 is a more typical phenomenon among young men than among young women.

\begin{tabular}{|c|c|c|c|c|c|c|}
\hline & \multirow{2}{*}{$\begin{array}{l}\text { All non- } \\
\text { completers }\end{array}$} & \multicolumn{2}{|c|}{ Gender } & \multicolumn{3}{|c|}{ Cohort of 16 -year-olds } \\
\hline & & Male & Female & 1993 & 1998 & 2003 \\
\hline \multicolumn{7}{|l|}{ Study-track cluster (cluster 1) } \\
\hline Denmark & 52.1 & 53.3 & 50.4 & 52.7 & 52.5 & 51.2 \\
\hline Finland & 41.2 & 42.9 & 38.8 & 35.0 & 42.1 & 46.1 \\
\hline Norway & 69.0 & 70.9 & 66.3 & 58.6 & 70.6 & 76.5 \\
\hline Sweden & 69.4 & 70.1 & 68.5 & 67.0 & 68.8 & 71.8 \\
\hline \multicolumn{7}{|l|}{ Major study-dominated clusters } \\
\hline Denmark (clusters 1, 8 \& 9) & 58.4 & 59.6 & 56.5 & 55.9 & 59.4 & 59.6 \\
\hline Finland (clusters 1, 5 \& 9) & 62.0 & 63.7 & 59.4 & 54.9 & 62.3 & 68.3 \\
\hline Norway (clusters 1, 6\& 10) & 79.7 & 81.3 & 77.4 & 73.7 & 80.2 & 84.4 \\
\hline Sweden (clusters 1 \& 5) & 69.9 & 70.4 & 69.2 & 67.8 & 69.2 & 72.1 \\
\hline \multicolumn{7}{|l|}{ Study- and employment clusters } \\
\hline Denmark (clusters 1, 2, 7, 8 \& 9) & 85.9 & 87.5 & 83.6 & 87.7 & 85.2 & 84.8 \\
\hline Finland (clusters 1, 3, 5, 6, 9\& 10) & 77.8 & 79.3 & 75.4 & 70.5 & 80.4 & 81.5 \\
\hline Norway (clusters $1,6,7,8$ \& 10) & 86.5 & 88.3 & 84.1 & 81.4 & 88.6 & 88.9 \\
\hline Sweden (clusters 1, 2, 5, 6 \& 7) & 80.7 & 80.3 & 80.9 & 79.2 & 82.4 & 80.3 \\
\hline \multicolumn{7}{|l|}{ NEET-dominated clusters } \\
\hline Denmark (clusters 3, 4, 5, 6 \& 10) & 14.2 & 12.6 & 16.6 & 12.4 & 14.8 & 15.3 \\
\hline Finland (clusters 2, 4, 7 \& 8) & 22.1 & 20.5 & 24.7 & 29.5 & 19.6 & 18.4 \\
\hline Norway (clusters 2, 3, 4, 5 \& 9) & 13.5 & 11.7 & 16.0 & 18.6 & 11.4 & 11.1 \\
\hline Sweden (clusters $3,4,8,9 \& 10$ ) & 19.4 & 20.6 & 19.1 & 20.8 & 17.7 & 19.9 \\
\hline
\end{tabular}

Notes: This table is compiled based on the information presented in the country-specific tables 3.6 to 3.9. NEET refers to Not in Employment, Education or Training. 
When adding to these study-track shares of non-completers their shares in employment-dominated tracks, Denmark almost closes the gap to Norway due to a remarkably large share of Danish non-completers following employment tracks upon completion of compulsory school. Put differently, Finland - closely followed by Sweden - comes out with the lowest shares of non-completers in study- and employment-dominated tracks in all dimensions under scrutiny: in total, by gender and by cohort. Conversely, Finland and Sweden have much larger shares of noncompleters following early tracks dominated by NEET activities. The high share of NEET-dominated tracks obtained for Finnish noncompleters reflects, in part, widespread unemployment among school dropouts, starting in the deep recession years of the early 1990s (cf. Table 3.7 above).

On the whole, we observe considerable heterogeneity across the four Nordic countries in the NEET trajectories followed by non-completers. This also explains the countries' different trends in NEET shares over cohorts, as well as the differently signed and sized gender gaps in the shares of non-completers following NEET-dominated tracks. Taken together, these observations justify our efforts to create both "common Nordic trajectories" and "stylized trajectories" in order to improve the cross-country comparability of results especially with respect to young non-completers. We start out, in the next chapter, from the common Nordic pathways. 


\section{School-to-work trajectories: common Nordic cluster results}

The previous chapter has shown that the trajectories through upper secondary education are, generally speaking, highly similar for young people in the four Nordic countries under study. Apart from certain country-specific particularities, the following picture emerges: a large majority of Nordic youth enters a study track after completing compulsory school and only a smaller share of them follows pathways dominated by activities outside both education and employment, i.e. so-called NEET activities. By and large, this holds true also for the category of noncompleters, that is, young people who have not completed an upper secondary education still at age 21 . Moreover, a comparison across cohorts reveals that there has, in all four Nordic countries, been a notable increase in the share of young people following study-dominated tracks. This convergence over time in early school-to-work trajectories is, in effect, discernible also among the non-completers. Put differently, increasing shares of also non-completers spend most, or all, of their time up to age 20 as full-time students, but without completing an upper secondary degree within five years after leaving compulsory school.

In this chapter, we have used one of the two methods described in sub-chapter 3.1 for forming cross-country comparable clusters of individual school-to-work trajectories. As explained in that sub-chapter, we have for each country made a list of the observed sequences of activities from age 16 up to age 20 and then calculated the number of young people following each of these tracks. Finally, this country-specific information has been pooled into one big data to which we have applied optimal matching in order to form ten clusters of sequences. This procedure also guarantees that sequences observed in more than one Nordic country are allocated in exactly the same way across the ten clusters. In this sense, the clusters formed are truly "common" for the four Nordic countries under study. Next we report results obtained using this method for the full youth population and separately for the young people identified to be non-completers still when aged 21 . 


\subsection{Common Nordic trajectories and clusters: all young people}

We start our presentation of results for common Nordic trajectories and clusters by providing, in Table 4.1, a general picture of how young people are distributed across the five main activities at each age point from 16 up to 20, when merging the country-specific information (based on all three youth cohorts) reported in sub-chapter $2.2 .^{6}$ As is evident from the table, and previously seen in Chapter 2, the share of full-time students undergoes a dramatic decline while the share in working life increases rapidly when Nordic youths approach the age of 20: by age 20, the share in employment (about $40 \%$ ) is almost as large as the share in full-time education (some 42\%). In total, these two shares cover close to $83 \%$ of 20-year-old Nordic youth, with the share in NEET activities thus being about $17 \%$. However, as can be seen from Table 4.1, this NEET share varies a lot over these early post-compulsory-school ages with strikingly high shares after three years in upper secondary education.

Table 4.1: Distribution (\%-share) of Nordic youth across main activities, by age

\begin{tabular}{lrrrrrr} 
Main activity & \multicolumn{9}{c}{ Age } & & $\begin{array}{r}\text { Average for age } \\
\mathbf{1 6} \text { to } \mathbf{2 0}\end{array}$ \\
\cline { 2 - 6 } & $\mathbf{1 6}$ & $\mathbf{1 7}$ & $\mathbf{1 8}$ & $\mathbf{1 9}$ & $\mathbf{2 0}$ & \\
Student & 90.3 & 90.1 & 83.4 & 43.8 & 42.2 & 70.0 \\
Employed & 1.6 & 3.7 & 8.4 & 36.3 & 40.4 & 18.1 \\
Student or employed & 91.9 & 93.8 & 91.8 & 90.1 & 82.6 & 88.1 \\
Unemployed & 0.6 & 1.2 & 3.1 & 9.3 & 9.0 & 4.6 \\
Pensioner (on disability benefits) & 0.4 & 0.5 & 0.6 & 1.0 & 1.2 & 0.7 \\
Other (inactivity) & 7.0 & 4.6 & 4.4 & 9.7 & 7.2 & 6.6 \\
NEET activities & 8.0 & 6.3 & 8.1 & 20.0 & 17.4 & 11.9 \\
Total & 100.0 & 100.0 & 100.0 & 100.0 & 100.0 & 100.0 \\
\hline
\end{tabular}

Note: The \%-shares are calculated from pooled information on individual post-compulsory-school trajectories up to age 20 , as produced separately for each country for all three youth cohorts combined, and reported in Chapter 2.

Figure 4.1, in turn, details the average information given in Table 4.1 by providing a cumulative graph of all sequences of young people's activities from age 16 up to age 20 . Since this graph is based on pooled infor-

${ }^{6}$ Here we repeat, once more, what was already underlined in the introductory chapter: for producing these common Nordic results, we do not merge individual-level data for the four Nordic countries under study. Instead we merely combine information on individual trajectories showing sequences of activities from age 16 up to age 20 . We have asked for and also received permission from the respective statistical bureaus to undertake such a pooling of country-specific individual trajectories. 
mation on the early school-to-work trajectories identified for each country, it actually represents a combination of the four country-specific graphs for all young people presented in sub-chapter 2.4. Accordingly, this particular figure shows individual school-to-work trajectories of more than 800,000 young persons. In view of this, it is not surprising that Figure 4.1 conveys much the same pattern as previously seen in Chapter 2, with large shares of Nordic youth following a study track of typically three or more years in upper secondary education straight after completing compulsory school, only occasionally after an initial year in non-study activities. Upper secondary education is followed by studies mostly in tertiary-level education, or shifts into working life or NEET activities. Next we take a closer look at these common Nordic school-to-work trajectories.

Figure 4.1: Common Nordic pathways: Individual school-to-work trajectories from age 16 up to age 20 for all young people in the four Nordic countries under study, as obtained from pooled information on all three youth cohorts under scrutiny

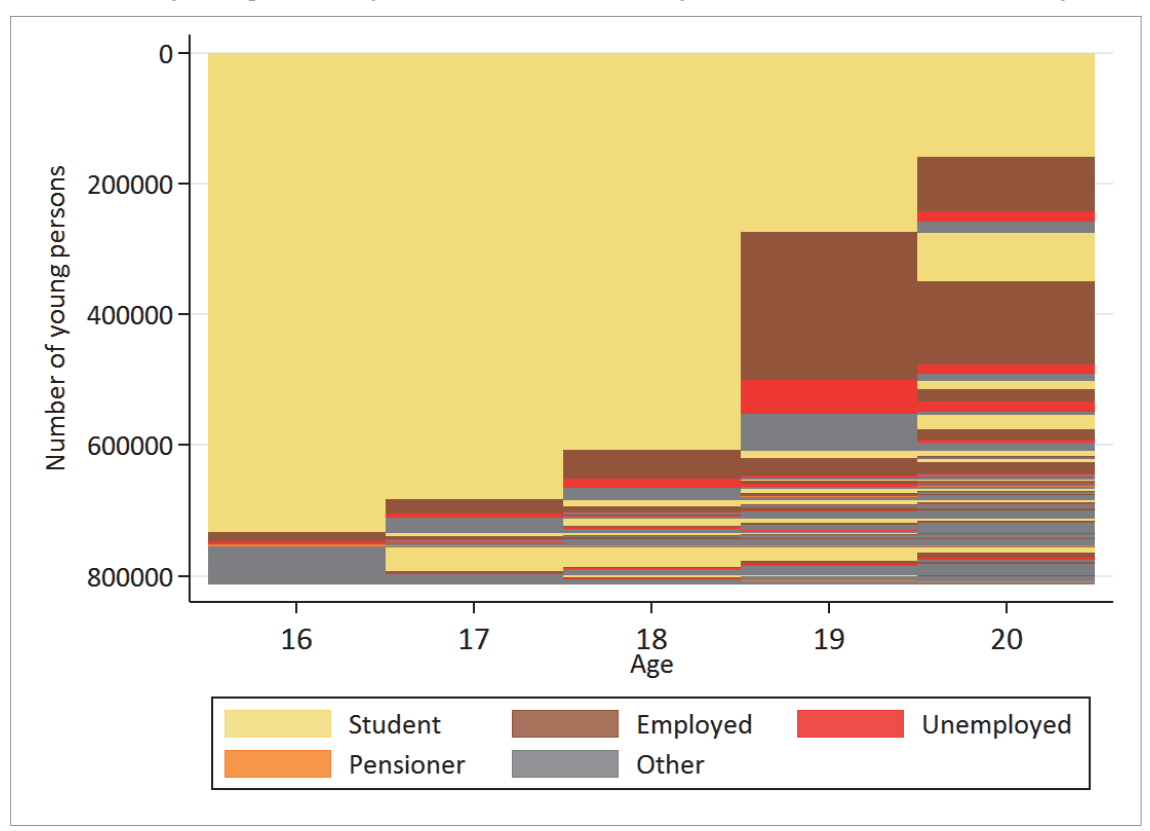

Notes: The graph is obtained by pooling the information on individual post-compulsory-school trajectories up to age 20 , as produced separately for each country and reported in Chapter 2 . The vertical axis gives the absolute number of individual school-to-work trajectories and, hence, the absolute number of young persons covered by the graph. The total number of young people covered by the graph is 802,867 ( 164,879 for Denmark, 193,567 for Finland, 156,164 for Norway and 290,257 for Sweden). 
The main types of trajectories followed by Nordic youth when moving through upper secondary education and into working life are illustrated in Figure 4.2 and broken down in Table 4.2. About $84 \%$ of young people in the four Nordic countries follow standard study tracks (cluster 1), that is, they typically spend at least three consecutive years in full-time education straight after completing compulsory school. The second largest group consists of young people who delay their entry into upper secondary education by one year (cluster 5).

The third and fourth largest groups represent trajectories where the young person spends a pause year from school when aged 17, either in working life (cluster 3) or in inactivity (cluster 2). However, this pause year is far from always followed by a return to education. Many young people prolong their spell in employment or in inactivity. For both groups of young people, there also seems to be a growing risk of their trajectories turning increasingly unstable after this pause year, as indicated by their frequent moves between employment, unemployment and inactivity. As can be seen from cluster 10, the fifth largest cluster, the risk of an early shift into NEET activities is typically even higher for those young people who start working straight after completing compulsory school.

Each of the other five clusters covers less than $2 \%$ of Nordic youth but, taken together, their coverage lands at 3.6\%, which makes them, in effect, the third largest group after standard and delayed study tracks. All these five clusters contain trajectories dominated by NEET activities. The spread of these types of tracks across no less than five clusters reveals, once more, the heterogeneity prevailing among youngsters facing notable problems when trying to continue in or return to postcompulsory education, and also when trying to move into working life. In particular, cluster 6 shows that prolonged spells outside both education and the labour market (in unknown activities) straight after completion of compulsory school result only occasionally in the young person re-entering school or moving into working life on a more permanent basis. Clusters 7, 8 and 9 are, in turn, dominated by spells in unemployment, with these spells increasingly interrupted by non-study, instead of study, activities when going from cluster 7 to cluster 9. Cluster 4, finally, contains trajectories followed by young people with health problems moving them into different types of disability arrangements.

Table 4.2 provides some more details on the ten clusters displayed in Figure 4.2. In line with the results reported in Chapter 3, Sweden comes out with by far the largest group of young people going straight into standard study tracks (about 91\%), followed by Norway (close to $86 \%$ ). 
Finland and especially Denmark have more differentiated tracks, with less than $80 \%$ of young people showing up in the study-track cluster (cluster 1). This difference between Sweden and the other three Nordic countries under study is due to a comparatively small share of young Swedes in principally all the other nine clusters. Most notably Sweden diverges from the other countries when it comes to cluster 3 , which contains trajectories characterised by a break spent in working life before returning to upper secondary education: while these types of early trajectories are quite uncommon among Swedish youth, they are relatively prevalent in Denmark, as are also post-compulsory-school tracks dominated by employment of a more permanent nature (cluster 10). Norway strikes out mainly with respect to a relatively large share of young people following trajectories characterised by frequent moves between education and unemployment (cluster 7). Finally, the most conspicuous feature of Finland is the high share of young people delaying their entry into upper secondary education (cluster 5). When it comes to the other clusters, most of which represent inactivity-dominated trajectories, no country comes out as a clear "outlier" when compared to the corresponding share in the other countries.

Also a comparison of cross-cohort distributions of young people into the ten clusters investigated provides further support for the observations made in Chapter 3 based on country-specific clusters. More precisely, as is evident in Table 4.2, the overwhelming dominance of standard study tracks (cluster 1 ) has been growing over time, covering about $86 \%$ of Nordic youth belonging to the youngest (2003) cohort. This growth in the share of young people following standard study tracks has occurred mainly at the expense of employment-dominated tracks (clusters 3 and 10): the combined share of these two clusters has declined from about $6 \%$ in the oldest (1993) cohort to $3 \%$ in the youngest (2003) cohort. 
Figure 4.2: Common Nordic clusters: Clustering of young people's school-to-work trajectories from age 16 up to age 20 into ten groups, using cluster analysis on pooled information for all three youth cohorts, all four Nordic countries combined

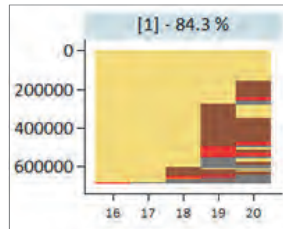

[5] $-4.6 \%$

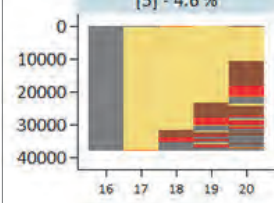

[9] $-0.4 \%$

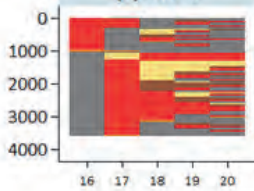

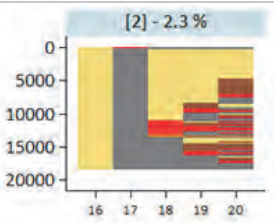

[6] $-1.6 \%$

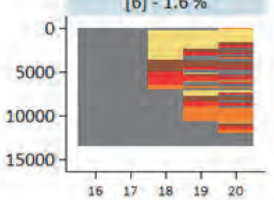

$[10]-2.0 \%$

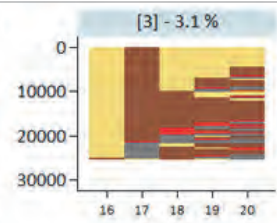

[7] $-0.9 \%$

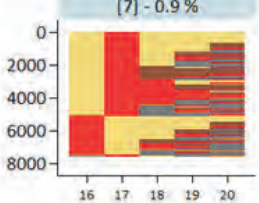

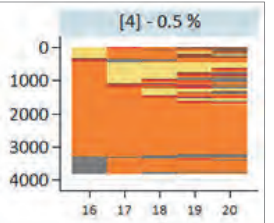

$[8]-0.2 \%$

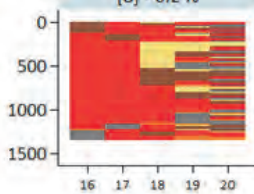

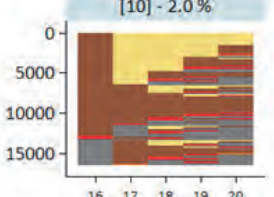

Notes: The clustering concerns the individual school-to-work trajectories displayed in Figure 4.1 above, using the optimal matching methodology described in sub-chapter 3.1. The vertical axis of each graph gives the absolute number of young persons in the cluster, whereas the percentage share on top of each graph shows the relative share of young people in the cluster. The number in bracketed parentheses on top of each graph refers to the number given by the algorithm creating the ten clusters by means of the subdivision procedure described sub-chapter 3.1.

Table 4.2: Distribution (\%-share) of young people across the ten common Nordic clusters displayed in Figure 4.2, by country and cohort

\begin{tabular}{lrrrrrrr}
$\begin{array}{l}\text { Cluster: } \\
\text { number - \%-share - label }\end{array}$ & \multicolumn{3}{c}{ Country } & \multicolumn{7}{c}{ Cohort of 16-year-olds } \\
& DK & FI & NO & SW & $\mathbf{1 9 9 3}$ & $\mathbf{1 9 9 8}$ & $\mathbf{2 0 0 3}$ \\
& & & & & & & \\
[1] - 84.3\% study tracks & 76.0 & 79.9 & 85.8 & 91.3 & 83.0 & 83.4 & 86.4 \\
[2] - 2.3\% study/inactivity/study tracks & 4.0 & 0.8 & 3.5 & 1.6 & 2.3 & 2.3 & 2.2 \\
[3] - 3.1\% study/employment/study tracks & 9.0 & 2.9 & 1.8 & 0.6 & 3.9 & 3.2 & 2.3 \\
[4] - 0.5\% disability tracks & 0.4 & 0.8 & 0.1 & 0.5 & 0.6 & 0.6 & 0.2 \\
[5] - 4.6\% inactivity-delayed-study tracks & 4.1 & 7.2 & 3.7 & 3.7 & 4.4 & 5.5 & 4.0 \\
[6] - 1.6\% inactivity/mix-of-activities tracks & 2.0 & 2.2 & 0.8 & 1.6 & 1.2 & 1.5 & 2.1 \\
[7] - 0.9\% study/unemployment/study tracks & 0.2 & 1.3 & 2.6 & 0.2 & 1.5 & 0.7 & 0.6 \\
[8] - 0.2\% unemployment tracks & 0.1 & 0.3 & 0.3 & 0.0 & 0.3 & 0.1 & 0.1 \\
[9] - 0.4\% inactivity/unemployment tracks & 0.2 & 0.9 & 0.7 & 0.1 & 0.6 & 0.4 & 0.3 \\
[10] - 2.0\% employment tracks & 4.0 & 3.6 & 0.8 & 0.5 & 2.2 & 2.2 & 1.7 \\
Total & 100 & 100 & 100 & 100 & 100 & 100 & 100 \\
\%-share in pooled Nordic trajectory-data & 20.6 & 24.1 & 19.4 & 35.9 & 32.8 & 32.6 & 34.7 \\
\hline
\end{tabular}

Notes: See Figure 4.2 above. 


\subsubsection{Main findings}

In this sub-chapter, we have reported the main results obtained when pooling country-specific information on young people's early postcompulsory-school trajectories into one big data and performing cluster analysis on this data to form ten groups (clusters) of trajectories, across which our Nordic youth are allocated by use of optimal matching. The reported results, which here concern all young people belonging to the three youth cohorts under scrutiny, provide strong support for the most important observations made on the basis of the country-specific cluster results presented in Chapter 3. This holds true for major similarities as well as key differences between the four Nordic countries under study.

In particular, large shares of young people in the four Nordic countries follow standard study tracks, implying that they continue in education straight after completing compulsory school and typically stay in full-time education for at least three years. This pattern is most pronounced for Sweden and least so for Denmark. In parallel, we observe much smaller but, nonetheless, conspicuous shares of young people (a) dropping out from post-compulsory education to move into working life; (b) delaying their entry into post-compulsory education by spending a break year working; or (c) moving into working life on a more permanent basis straight after completing compulsory school. These employment-dominated tracks are strikingly common among young Danes. A comparison across cohorts reveals, however, that study tracks have gained in popularity among compulsory-school leavers, mainly at the expense of employment tracks. Not surprisingly, this trend is weakest for Denmark.

Additionally, we see non-negligible shares of compulsory-school leavers following trajectories dominated by NEET activities. All these trajectories tend to involve a high risk of the young person being marginalised from economic as well as social life. Moreover, as shown in Chapter 3, these NEET-dominated trajectories are observable mainly among the non-completers, i.e. young people having only a compulsoryschool exam still at age 21. Next we, therefore, turn our focus to these non-completers using a pan-Nordic perspective. 


\subsection{Common Nordic trajectories and clusters: non- completers}

Again, we start our presentation of results for common Nordic trajectories and clusters by providing a general picture of how young people in the four Nordic countries are distributed across the five main activities at each age point from 16 up to 20 (Table 4.3). However, now the emphasis is on non-completers, implying that the subsequent results are obtained by excluding all completers from the pooled Nordic data used in the previous sub-chapter. In particular, we form a new set of ten clusters based only on information about the non-completers' early postcompulsory-school trajectories up to age 20. In this context, it is therefore important to recall that the non-completion rates at age 21 differ considerably across the four countries, as shown in Table 2.2 of Chapter 2. This means that relatively larger shares of young Danes and Norwegians than of young Finns and Swedes enter this combined Nordic data on non-completers' educational and labour market experiences when aged 16 to 20 .

\begin{tabular}{|c|c|c|c|c|c|c|}
\hline \multirow[t]{2}{*}{ Main activity } & \multicolumn{5}{|c|}{ Age } & \multirow{2}{*}{$\begin{array}{r}\text { Average for age } 16 \\
\text { to } 20\end{array}$} \\
\hline & 16 & 17 & 18 & 19 & 20 & \\
\hline Student & 75.2 & 69.4 & 54.5 & 37.9 & 32.4 & 53.9 \\
\hline Employed & 4.0 & 10.6 & 18.2 & 30.0 & 36.0 & 19.8 \\
\hline Student or employed & 79.2 & 80.0 & 72.7 & 67.9 & 68.4 & 73.7 \\
\hline Unemployed & 2.1 & 4.3 & 9.3 & 12.1 & 12.7 & 8.1 \\
\hline Pensioner(on disability benefits) & 1.2 & 1.2 & 1.9 & 3.3 & 4.2 & 2.4 \\
\hline Other (inactivity) & 17.6 & 14.4 & 16.1 & 16.6 & 14.7 & 15.9 \\
\hline NEET activities & 20.9 & 20.1 & 26.3 & 32.0 & 31.6 & 26.4 \\
\hline Total & 100.0 & 100.0 & 100.0 & 100.0 & 100.0 & 100.0 \\
\hline
\end{tabular}

Notes: The \%-shares are calculated from pooled information on individual post-compulsoryschool trajectories up to age 20 , as produced separately for each country's non-completers and reported in Chapter 2.

As shown in Table 4.3, and in line with what was observed in Chapter 2, large shares of non-completers continue in education straight after completing compulsory school. More than one-half of them are full-time students still at age 18, but less than one-third two years later, at age 20. Concomitantly, the share of non-completers outside both education and working life (in NEET activities) increases steadily, from $21 \%$ at age 16 to $32 \%$ at age 19 , a share that is approximately unchanged one year later, at age 20. This increase is, however, almost entirely due to growing shares of non-completers in unemployment and on disability arrangements, while their share in inactivity ("other") changes only marginally 
during these first five years after completion of compulsory school. Simultaneously, also the share of non-completers in working life grows rapidly, especially after age 18 . By age 20 , approximately one-third of the non-completers are in education, one-third in employment and onethird in NEET activities.

After this brief look at the average age-specific distributions of Nordic non-completers across the five main activities under scrutiny, we turn our focus to the overall shape of these youngsters' individual postcompulsory-school trajectories up to age 20. This information is given in Figure 4.3, which provides a cumulative graph of all sequences of noncompleters' activities between age 16 and 20. As for the pooled youth populations analysed in the previous sub-chapter, also this graph is in reality a combination of the four country-specific graphs for noncompleters presented and discussed in sub-chapter 2.4. When pooling all this country-specific information on non-completers' early school-towork trajectories, we end up with individual school-to-work trajectories of almost 200,000 young Nordic non-completers. Accordingly, it is hardly surprising that Figure 4.3 mediates a pattern that was observed already based on the country-specific results of Chapter 2 .

In particular, the early school-to-work trajectories of non-completers displayed in Figure 4.3 form a "staircase" with young people leaving post-compulsory-school in a more or less smooth "step-wise" way, mainly after one, two or three years in post-compulsory education. Fewer of them are likely to leave after four years in full-time education. Instead they often continue in post-compulsory education still at age 20 but without having completed an upper secondary degree still by age 21 . Some dropouts soon return to education while others stay in non-study activities. Moreover, this same pattern seems to hold true also for those non-completers who delay their start in post-compulsory education by spending typically one year outside both education and the labour market (located at the bottom of the graph).

Figure 4.3 also indicates that the probability of non-completers getting a job upon leaving post-compulsory education increases, and of ending up in NEET activities decreases with the number of years spent in post-compulsory education before dropping out. In other words, more years in upper secondary education, even without completing a degree, seem to be related to less risky school-to-work profiles up to age 20 . 
Figure 4.3: Common Nordic pathways: Individual school-to-work trajectories from age 16 up to age 20 for all non-completers in the four Nordic countries under study, as obtained from pooled information on all three youth cohorts under scrutiny

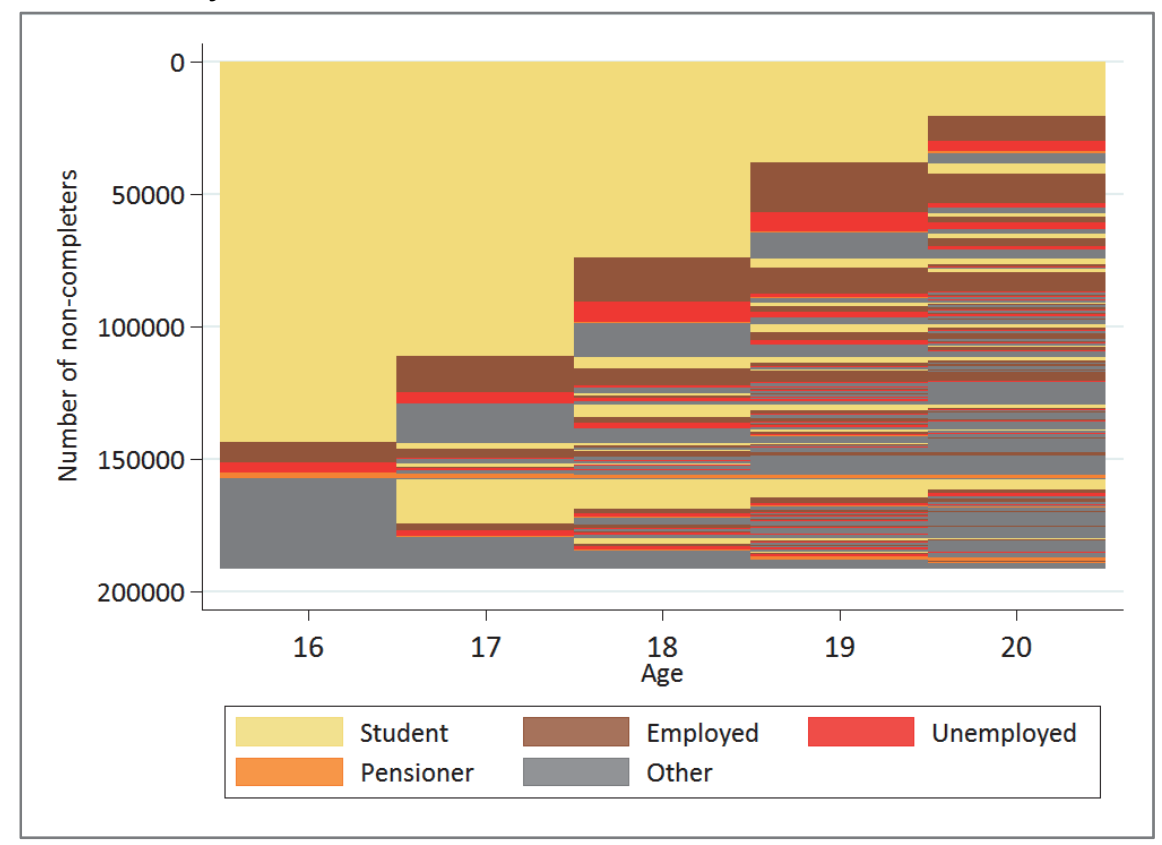

Notes: The graph is obtained by pooling the information on non-completers' individual postcompulsory-school trajectories up to age 20 , as produced separately for each country and reported in Chapter 2. The vertical axis gives the absolute number of individual school-to-work trajectories and, hence, the absolute number of young non-completers covered by the graph. The total number of young people covered by the graph is 189,804 (61,884 for Denmark, 34,956 for Finland, 46,441 for Norway and 46,523 for Sweden). Non-completers refer to young people who still five years after completing compulsory education have no upper secondary degree.

The main types of early post-compulsory-school trajectories followed by Nordic non-completers are displayed in Figure 4.4 with more details on each of the ten clusters given in Table 4.4. Also the non-completers are heavily concentrated into the study-track cluster (cluster 1): almost three out of five Nordic non-completers follow a study track straight upon having left compulsory school. Moreover, also among the noncompleters we observe a growth in the share following standard study tracks, implying that increasingly larger shares of the non-completers continue directly in post-compulsory education for typically three or more years, but without completing an upper secondary degree within five years after completing compulsory school.

In view of the strong dominance of standard study tracks among Swedish youth, it is hardly surprising that also young Swedes with difficulties in completing an upper secondary degree continue in large 
shares in post-compulsory education. While two-thirds of Swedish noncompleters show up in standard study tracks, the corresponding share is only about $43 \%$ among Finnish non-completers, with Denmark and Norway falling between these two extremes (Table 4.4). However, and as pointed out also earlier, it is more common among young Finns than among other Nordic youth to delay their entry into post-compulsory education with typically one year (cluster 9). These late starters comprise as much as $14.3 \%$ of the Finnish non-completers compared to only $5 \%$ in Denmark. When combining the shares of these two study-trackdominated clusters ( 1 and 9), we cover close to 58\% of Finnish noncompleters. But even with this share, Finland still ranks below Denmark (62.4\%) and far below Norway (71.4\%) and Sweden (75.9\%).

Another common set of non-completers' trajectories involves frequent spells in working life, either on a temporary or, so it seems, a more permanent basis (clusters 6 and 7). A major difference between these two clusters is that cluster 6 contains employment-dominated trajectories interrupted mainly by years spent in full-time education, whereas in cluster 7 , these education breaks are mostly replaced by time spent in NEET activities. In view of cluster 7 thus containing much more risky trajectories, it is confident to note that it covers, on average, only $2.4 \%$ of Nordic non-completers compared to $11 \%$ for cluster 6 . However, the time trend for these two clusters is less satisfying: while there has been a decline across cohorts in the share of non-completers going into the less risky trajectories of cluster 6 , the corresponding share for the more risky trajectories of cluster 7 reveals a slight increase (Table 4.4).

As is only to be expected based on previously presented results, the study-employment-mixed tracks of cluster 6 are most common among Danish non-completers, covering almost one-fifth of them. The share of non-completers going into cluster 6 is relatively high also in Finland (almost 12\%), but quite low in Norway (5.4\%) and Sweden (3.6\%). When it comes to the more risky post-compulsory-school employment profiles contained in cluster 7 , the situation looks quite different with the highest share observed for Finnish non-completers (4.4\%), followed by Danish non-completers (3.1\%), but very small shares for Swedish $(1.4 \%)$ and Norwegian $(0.7 \%)$ non-completers.

Relatively large shares of non-completers drop out after one year in post-compulsory education and move into NEET activities, either into unemployment (cluster 2) or into activities outside both education and the labour market (cluster 3). Although a non-negligible share of these early dropouts return to education either on a temporary or more permanent basis, most of them seem to continue on bumpy tracks with fre- 
quent shifts between non-study activities. The dropout-into-inactivity tracks of cluster 3 have retained, or even strengthened, their position across cohorts with a share of $7.2 \%$ in the oldest (1993) cohort and $7.7 \%$ in the youngest (2003) cohort. These types of trajectories are most common among Danish non-completers, but quite prevalent also among Norwegian $(8.7 \%)$ and Swedish $(6 \%)$ non-completers. The dropout-intounemployment tracks of cluster 2 cover a notably smaller share of Nordic non-completers (2.2\%). Moreover, the prevalence of this type of tracks has declined over time with only $1.4 \%$ of the non-completers in the youngest (2003) cohort following such tracks. While these tracks are of equal importance in Finland and Norway, covering close to 5\% of the two countries' non-completers, they play a marginal role among Danish and Swedish non-completers.

Figure 4.4: Common Nordic clusters: Clustering of young non-completers'schoolto-work trajectories from age 16 up to age 20 into ten groups, using cluster analysis on pooled information for all three youth cohorts, all four Nordic countries combined

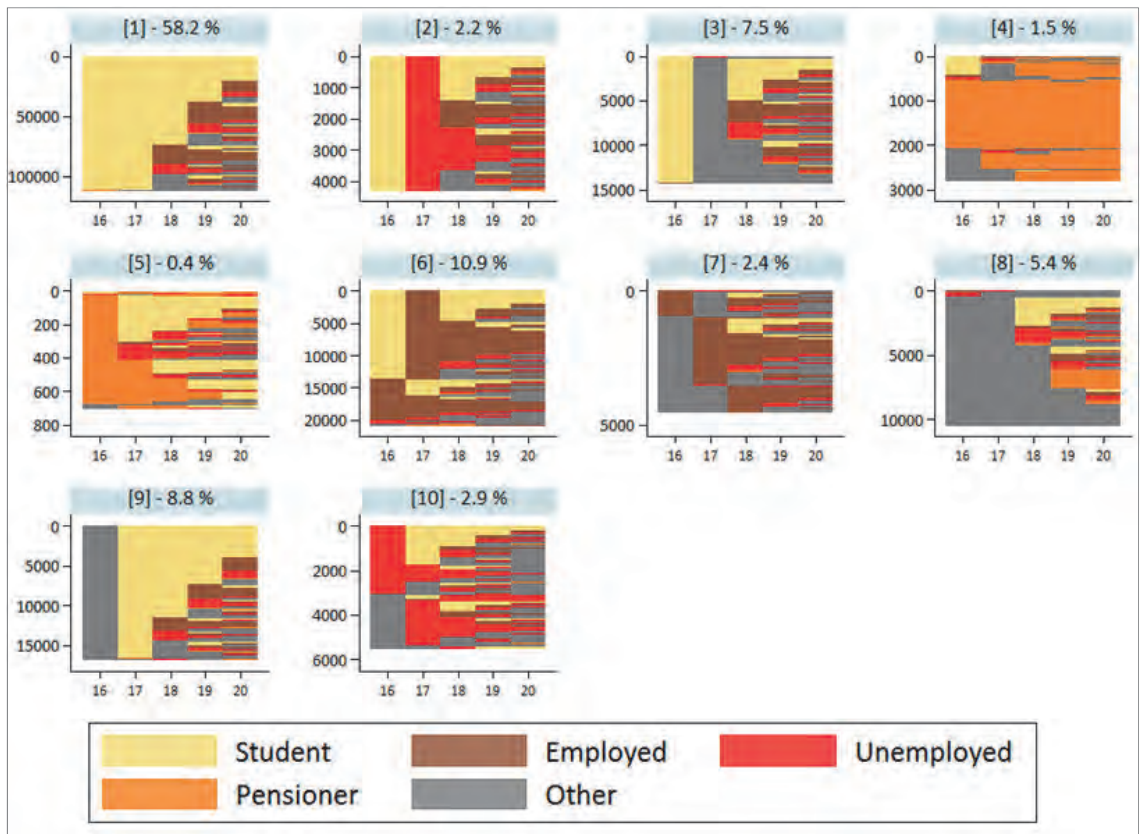

Notes: The clustering concerns the individual school-to-work trajectories of non-completers displayed in Figure 4.4 above, using the optimal matching methodology described in sub-chapter 3.1. The vertical axis of each graph gives the absolute number of young persons in the cluster, whereas the percentage share on top of each graph shows the relative share of young people in the cluster. The number in bracketed parentheses on top of each graph refers to the number given by the algorithm creating the ten clusters by means of the subdivision procedure described sub-chapter 3.1. Non-completers refer to young people who still five years after completing compulsory education have no upper secondary degree. 
Cluster 10, covering on average close to $3 \%$ of Nordic non-completers, is related to both cluster 2 and cluster 3 in the sense that also these tracks involve frequent spells in NEET activities. A major difference, however, is that the non-completers going into cluster 10 do not drop out from post-compulsory education but, instead, move into NEET activities straight after completing compulsory school. Another conspicuous difference is that the tracks of cluster 10 are much more seldom interrupted by spells in education or employment. On the contrary, the trajectories of cluster 10 seem to contain ever more time spent outside not only education but also the labour market when these youngsters approach the age of 20. While the share of non-completers following this type of track has declined over time, these high-risk pathways have remained more prevalent among Finnish and Norwegian non-completers than among Danish and Swedish non-completers.

Cluster 8 contains youngsters spending most, or all, of their first five years after completing compulsory school outside both education and the labour market, that is, in unknown activities (inactivity). These prolonged spells in inactivity early in life may, so it seems, turn into highly different subsequent activities. In other words, the outcome of these initial inactivity spells is quite mixed when looking at the situation at age 19 or age 20 . Nonetheless, it seems as if shorter initial spells in inactivity are more likely to end in a return to education or a shift into working life, whereas prolonged spells in inactivity tend to involve a higher risk of the young person continuing in NEET activities. While cluster 8 covers, on average, $5.4 \%$ of Nordic non-completers, its share is clearly higher (over 8\%) in both Finland and Sweden. Moreover, a comparison across cohorts reveals that it contains a much larger share of the noncompleters in the youngest (2003) cohort as compared to the oldest (1993) cohort (7.3\% vs. 4.1\%), implying that growing shares of noncompleters disappear for several years from all large administrative registers straight after completing compulsory school. 
Table 4.4: Distribution (\%-share) of young non-completers across the ten common Nordic clusters displayed in Figure 4.4, by country and cohort

\begin{tabular}{|c|c|c|c|c|c|c|c|}
\hline \multirow{2}{*}{$\begin{array}{l}\text { Cluster: } \\
\text { number - \%-share - label }\end{array}$} & \multicolumn{4}{|c|}{ Country } & \multicolumn{3}{|c|}{ Cohort of 16 -year-olds } \\
\hline & DK & $\mathbf{F I}$ & No & sw & 1993 & 1998 & 2003 \\
\hline [1] $58.2 \%$ study tracks & 57.4 & 43.3 & 63.2 & 65.9 & 55.4 & 57.5 & 61.3 \\
\hline [2] $2.2 \%$ study/unemployment/mix tracks & 0.3 & 4.7 & 4.6 & 0.8 & 3.9 & 1.6 & 1.4 \\
\hline [3] $7.5 \%$ study/inactivity/mix tracks & 10.2 & 3.1 & 8.7 & 6.0 & 7.2 & 7.4 & 7.7 \\
\hline [4] $1.5 \%$ disability tracks & 0.5 & 2.5 & 0.3 & 2.9 & 1.7 & 2.0 & 0.7 \\
\hline [5] $0.4 \%$ disability-delayed-study tracks & 0.0 & 1.4 & 0.2 & 0.3 & 0.7 & 0.4 & 0.0 \\
\hline [6] $10.9 \%$ study/employment/study tracks & 19.5 & 11.8 & 5.4 & 3.6 & 12.3 & 11.4 & 9.1 \\
\hline [7] $2.4 \%$ inactivity/employment tracks & 3.1 & 4.4 & 0.7 & 1.4 & 1.9 & 2.7 & 2.4 \\
\hline [8] $5.4 \%$ prolonged-inactivity tracks & 3.5 & 8.9 & 2.5 & 8.1 & 4.1 & 4.8 & 7.3 \\
\hline [9] $8.8 \%$ inactivity-delayed-study tracks & 5.0 & 14.3 & 8.2 & 10.0 & 8.3 & 9.8 & 8.2 \\
\hline [10] $2.9 \%$ unemployment/inactivity-mix tracks & 0.4 & 5.6 & 6.1 & 1.0 & 4.4 & 2.5 & 1.9 \\
\hline Total & 100 & 100 & 100 & 100 & 100 & 100 & 100 \\
\hline $\begin{array}{l}\% \text {-share in non-completers' pooled Nordic } \\
\text { school-to-work-trajectory data }\end{array}$ & 32.9 & 19.2 & 22.9 & 24.9 & 31.3 & 33.8 & 35.0 \\
\hline
\end{tabular}

Notes: See Figure 4.4 above.

The two final clusters are dominated by disability arrangements, covering together about $2 \%$ of Nordic non-completers. Both clusters are dominated by prolonged spells on disability benefits straight after completion of compulsory school. Most young people experiencing early health problems tend to continue on disability benefits (cluster 4) while only a small share of them succeed in returning to education, either permanently or only for a year or two (cluster 5). The uninterrupted disability tracks contained in cluster 4 are most common among Finnish and Swedish non-completers, but show a declining trend over time, obviously mainly due to stricter eligibility conditions for young people. The disability-delayed study tracks of cluster 5 occur mainly among Finnish noncompleters, but these types of trajectories have basically disappeared among non-completers belonging to younger cohorts.

\subsubsection{Main findings}

This analysis of common Nordic trajectories and clusters for noncompleters largely confirm the main findings concerning cross-country similarities and dissimilarities pointed out in Chapter 3 based on separate clustering of non-completers for the four Nordic countries under study. The most distinct feature of Nordic non-completers is that large and increasing shares of them continue directly in post-compulsory education and spend most, or all, of their time up to age 20 as full-time students, but without completing an upper secondary degree by age 21 . This pattern is most prevalent among Swedish non-completers and least prevalent among Finnish non-completers. Moreover, this overall cross- 
country pattern does not change, when adding the shares of noncompleters continuing in post-compulsory education only after first having spent typically one year in non-study activities, and mostly in activities not showing up in any large administrative register. The share of non-completers following these types of inactivity-delayed study tracks has remained approximately unchanged across cohorts and is highest among Finnish non-completers.

Compared to these study tracks, the other trajectories play a much smaller role, covering notably lower shares of Nordic non-completers. Broadly speaking, we can identify two major categories. The first category contains various types of employment-dominated trajectories with the move into working life occurring immediately after compulsory school, after early dropout from post-compulsory education, or after a year in inactivity straight after completion of compulsory school. Prolonged employment tracks as well as employment-dominated tracks involving frequent returns to education are common especially among Danish non-completers but also among Finnish non-completers. However, the share of such tracks among the non-completers reveals a declining trend over time. Early employment tracks coupled with frequent spells in inactivity are much less common, but reveal a slowly increasing trend. These bumpy employment tracks are most common among Finnish non-completers.

The second category consists of non-completers following highly different early post-compulsory trajectories, a common feature of which is that they for the most part represent high-risk tracks. In other words, they are dominated by NEET activities which follow upon an early dropout from post-compulsory education, or into which the young person moves straight after completing compulsory school. Only occasionally do these tracks involve a return to education even temporarily. Moreover, the share of non-completers following tracks dominated by NEET activities has remained practically unchanged over cohorts. A comparison across the four Nordic countries implies, in turn, that the share of noncompleters finding themselves in high-risk NEET-dominated tracks is much lower in Denmark than in the other three countries. Hence, once more we are inclined to conclude that Danish non-completers seem to fair better than Finnish, Norwegian and Swedish non-completers. Whether this holds true also beyond the age of 20 is an issue that we return to in Chapters 6 and 7.

On the other hand, there is reason to recall also in this context that the share of young non-completers differs considerably across the four Nordic countries under study (see Chapter 2). While the summary in 
Table 4.4 illustrates well the situation among young non-completers in the different countries, it overlooks the fact that these percentage shares cover, in effect, highly varying proportions of the full youth population in each country. In Table 4.5 we, therefore, recalculate the distribution of young non-completers in Table 4.4 by relating each percentage share to the full youth population of the country in question.

Table 4.5: Distribution of young non-completers across the ten common Nordic clusters displayed in Figure 4.4 , by country, \%-share of the full youth population

\begin{tabular}{lrrrr} 
Cluster: & \multicolumn{3}{c}{ \%-share of the full youth population } \\
number - label & Denmark & Finland & Norway & Sweden \\
& & & & \\
& & 7.8 & 18.8 & 10.5 \\
[1] study tracks & 0.1 & 0.8 & 1.4 & 0.1 \\
[2] study/unemployment/mix tracks & 3.8 & 0.6 & 2.6 & 1.0 \\
[3] study/inactivity/mix tracks & 0.2 & 0.5 & 0.1 & 0.5 \\
[4] disability tracks & 0.0 & 0.3 & 0.1 & 0.0 \\
[5] disability-delayed-study tracks & 7.3 & 2.1 & 1.6 & 0.6 \\
[6] study/employment/study tracks & 1.2 & 0.8 & 0.2 & 0.2 \\
[7] inactivity/employment tracks & 1.3 & 1.6 & 0.7 & 1.3 \\
[8] prolonged-inactivity tracks & 1.9 & 2.6 & 2.4 & 1.6 \\
[9] inactivity-delayed-study tracks & 0.1 & 1.0 & 1.8 & 0.2 \\
[10]unemployment/inactivity-mix tracks & 37.3 & 18.0 & 29.7 & 16.0 \\
Non-completers' share in the full youth population & & & & \\
\hline
\end{tabular}

Notes: The percentage shares displayed in Table 4.4 as recalculated in relation to the full youth population of each country.

Due to the prevailing cross-country differences in non-completion rates among the 21-year-olds, this rescaling is bound to produce a picture that differs from the one emerging in Table 4.4. We now observe that those young non-completers having followed study-dominated tracks upon completion of compulsory school cover nearly one-third of the Danish and more than one-fifth of the Norwegian youth population. The corresponding share for both Finland and Sweden is only about $10 \%$. Furthermore, about $6 \%$ of Finnish as well as Norwegian youths have followed one of the four inactivity-dominated tracks (track [7] to [10]), compared to, respectively, only 3.3 and $4.5 \%$ for Sweden and Denmark. A common feature of the four countries, however, is that disability tracks are followed by approximately the same proportion of youth in all four countries.

All in all, when relating the proportion of young non-completers having followed different tracks upon completion of compulsory school to the full youth population, the overall pattern changes in certain respects. As noted above, the reason is simple: young non-completers make up highly different shares of the full youth population in the four Nordic countries under study. Thus, the relatively large share of 21-year-old non-completers in Denmark implies that the proportion of Danish youth 
following standard study tracks or study/employment tracks straight after completing compulsory school - but without completing an upper secondary degree by age 21 - is much larger in Denmark than in the other three countries. This rescaling in proportion to the full youth population also shows that the situation in Norway resembles more that in Denmark than the picture emerging for Finland and Sweden, especially when it comes to a large share of youngsters continuing in education but without completing an upper secondary degree by age 21 . With Finland, in turn, Norway shares the feature of having a relatively large group of youngsters turning into young non-completers because they end up outside both education and the labour market already at an early age. 



\section{Non-completers' school-to- work trajectories: stylized cluster results}

In the previous chapter, country-specific information on individual school-to-work trajectories was pooled into one big data. Based on all this information for the four Nordic countries under study, ten common Nordic clusters were formed for all young people and separately for the non-completers. The results obtained for the common Nordic clusters presented in Chapter 4 provided further support for the broad picture painted already by the country-specific cluster results reported in Chapter 3. Basically two distinct features emerged. First, large shares of young people in the Nordic countries follow study tracks straight after completing compulsory school, occasionally only after a break year before entering post-compulsory education. The rest of the identified clusters of typical school-to-work trajectories play a more marginal role as alternatives to standard study tracks in the sense that they cover much smaller, but by no means negligible, shares of young people. Second, while standard study tracks are often followed also by non-completers, these alternative types of tracks play a much more important role among young people facing problems in completing an upper secondary degree, let alone starting an upper secondary education.

While there are striking similarities between the four Nordic countries in all these respects, they reveal distinct differences, as well. In this chapter, we take a closer look at these differences, but also similarities, when it comes to the early post-compulsory-school trajectories followed by Nordic non-completers. This is done by use of clustering by reference sequences, a method outlined in some detail in Chapter 3. In brief, the basic idea is to choose a number of reference sequences and cluster the other sequences around these reference sequences. By use of the same reference sequences for all four Nordic countries, we are able to shed further light on both similarities and dissimilarities across the four countries in relation to non-completers' post-compulsory-school experiences up to age 20 . 


\subsubsection{6 stylized pathways}

For the cluster analysis reported in this chapter, we constructed a total of 16 reference sequences. We prefer to call these reference sequences "stylized pathways", as we have explicitly aimed at identifying reference sequences for non-completers' main post-compulsory-school activities up to age 20 which resemble as closely as possible the "typical" trajectories of non-completers distinguished both in the country-specific cluster analysis of Chapter 3 and in the common Nordic cluster analysis of Chapter 4. Two main criteria were used when selecting these stylized pathways. A first criterion was: For how long do the young non-completers stay initially in post-compulsory education? A second criterion was, logically: What is their main activity if leaving school? Based on different combinations of these two criteria, we constructed a total of 16 stylized pathways for the non-completers. Thereafter, we allocated all non-completers to the stylized pathway that looked most similar to the trajectory they had actually followed upon completion of compulsory school, up to age 20. Hence, the only difference in this setting, when compared to the clustering undertaken in Chapters 4 and 5 is that here, the clusters are pre-determined (by us) and not decided by the clustering method used.

Table 5.1 presents the 16 stylized pathways constructed based on the two aforementioned criteria, grouped after the non-completer's main activity after leaving upper secondary education: continue in education, employment, unemployment, disability arrangements or "other" (i.e. unknown inactivity). The number series in the column labelled "stylized pathways" shows the pre-determined reference sequences of main activities from age 16 up to age 20. Each number signifies a main activity, defined in the same way as in the previous chapters, that is, with 1 standing for full-time student, 2 for being employed, 3 for being unemployed, 4 for receiving disability benefits, and 5 for being in the dumping category "other". The order of these numbers denotes the year in which the activity takes place. For instance, the stylized pathway [11122] means that the young person spends the first three years, from age 16 to age 18, in post-compulsory education. Thereafter, this person moves into working life and stays employed up to age 20. This employment spell may, of course, continue beyond age 20 - as might any of the other main activities. Also in this chapter, however, we stop our tracing of young people's activities at age 20. Only in the next chapters will we investigate what happens beyond the age of 20 .

Next we turn to the clusters formed when allocating all noncompleters across these 16 stylized pathways, separately for each country. As already pointed out above, this means that all non-completers 
show up in one of the 16 clusters built around these stylized pathways, depending on which pathway comes closest to the non-completer's actual post-compulsory-school trajectory. We present these results by following the categorization of pathways used in Table 5.1. In other words, we start from the first category containing stylized educational pathways, that is, two typical post-compulsory-school trajectories dominated by noncompleters spending practically all their time up to age 20 as full-time students (but without obtaining an upper secondary certificate by age 21).

\begin{tabular}{|c|c|c|}
\hline $\begin{array}{l}\text { Main activity after leaving } \\
\text { education (criterion 2) }\end{array}$ & $\begin{array}{l}\text { Years in post-compulsory education up to } \\
\text { age } 20 \text { (criterion 1) }\end{array}$ & Stylized pathways \\
\hline Continue in education & $\begin{array}{l}\text { all } 5 \text { years in education } \\
\text { delayed start, all } 4 \text { years in education }\end{array}$ & $\begin{array}{l}{[11111]} \\
{[51111]}\end{array}$ \\
\hline Employment & $\begin{array}{l}\text { first } 3 \text { years in education } \\
\text { first } 2 \text { years in education } \\
\text { first (initial) year in education } \\
\text { no entry into post-compulsory education }\end{array}$ & $\begin{array}{l}{[11122]} \\
{[11222]} \\
{[12222]} \\
{[52222]}\end{array}$ \\
\hline Unemployment & $\begin{array}{l}\text { first } 3 \text { years in education } \\
\text { first } 2 \text { years in education } \\
\text { first (initial) year in education }\end{array}$ & $\begin{array}{l}{[11133]} \\
{[11333]} \\
{[13333]}\end{array}$ \\
\hline Disability benefits (pensioner) & $\begin{array}{l}\text { first } 3 \text { years in education } \\
\text { first } 2 \text { years in education } \\
\text { first (initial) year in education }\end{array}$ & $\begin{array}{l}{[11144]} \\
{[11444]} \\
{[14444]}\end{array}$ \\
\hline Other (inactivity) & $\begin{array}{l}\text { first } 3 \text { years in education } \\
\text { first } 2 \text { years in education } \\
\text { first (initial) year in education } \\
\text { no entry into post-compulsory education }\end{array}$ & $\begin{array}{l}{[11155]} \\
{[11555]} \\
{[15555]} \\
{[55555]}\end{array}$ \\
\hline
\end{tabular}

Notes: 1 = full-time student, 2 = employed, 3 = unemployed, 4 = disability beneficiary, 5= "other". For other explanations of the table, see the text.

\subsubsection{Stylized educational pathways}

The country-specific graphs contained in Figure 5.1 illustrate what the two stylized educational pathways actually look like in the four Nordic countries, and also how prevalent they are in each country. As before, the vertical axis of each graph gives the absolute number of young people covered by the graph, whereas the information on top of each graph shows the stylized pathway in question and the share of each country's non-completers going into that particular cluster. Hence, these \%-shares of the 16 stylized pathways are country specific and, accordingly, sum up to $100 \%$ for each country. Some more details, by gender and cohort, are provided in the accompanying table (Table 5.2).

Figure 5.1 confirms the overall picture for non-completers mediated by the results presented in Chapters 3 and 4: large shares of also noncompleters continue, either directly or after a break year, in post- 
compulsory education. In particular, these two sets of country-specific graphs amplify this overall picture by explicitly illustrating the shares of non-completers spending all or most of their time, from age 16 up to age 20 , as full-time students. Indeed, these two stylized educational pathways can, in effect, be seen as extreme cases of the more general studytrack clusters formed in Chapters 3 and 4 in the sense that they identify non-completers that are highly engaged in post-compulsory studies but, nonetheless, fail to achieve an upper secondary degree within five years after completing compulsory school. As can be seen from Figure 5.1, the share of these full-time-studying non-completers is highest in Denmark (35\%), closely followed by Norway and Sweden, and lowest in Finland (close to 22\%). When adding the share of non-completers delaying their entry into post-compulsory education, the two stylized educational pathways cover almost $40 \%$ of the Danish non-completers and $35-37 \%$ of the non-completers in the other three countries. Again, Finland "closes the gap" to the other three countries due to a comparatively high share of late starters $(13.5 \%)$.

Figure 5.1: Stylized educational pathways of non-completers, by country

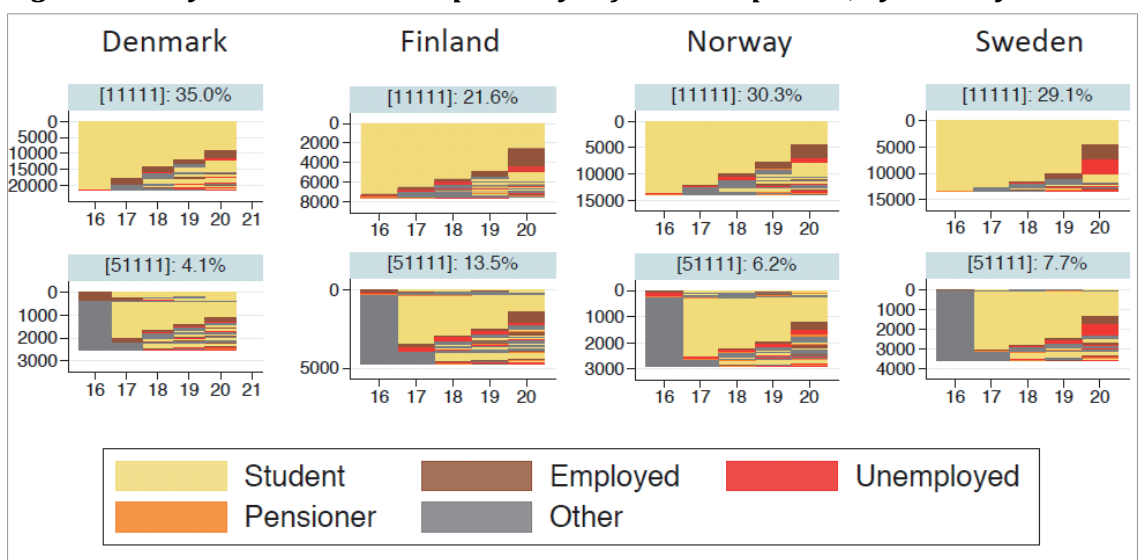

Notes: The clustering concerns individual school-to-work trajectories of non-completers when aged 16 to 20 , using clustering by reference sequences as described in sub-chapter 3.1. The vertical axis of each graph gives the absolute number of non-completers in the cluster, whereas the percentage share on top of each graph shows the relative share of non-completers in the cluster, by country. The country-specific percentage shares for the 16 stylized pathways thus sum up to $100 \%$ for each country. The number in bracketed parentheses on top of each graph refers to the stylized pathway in question as described in Table 5.1. For more details, by gender and cohort, see Table 5.2 below.

This full, or close to full, engagement in education over the five years following upon completion of compulsory school reveals no clear-cut gendered pattern across the four countries, as is evident from Table 5.2. In Norway and especially in Denmark, young men are more likely 
than young women to continue in post-compulsory education for most or all years up to age 20, but without completing an upper secondary degree by age 21 . The share in these two countries of educationengaged non-completers delaying their entry into full-time education is small, covering an approximately equal share of young men and young women. In Finland and Sweden, on the other hand, the situation is the opposite, except for Swedish late starters. These cross-country differences in gendered shares do not change when adding up the noncompleter shares of the two stylized educational pathways, as shown in the lower half of Table 5.2.

These gender-specific findings are interesting in view of the country results for non-completers reported in Chapter 3: for all four countries, we observed that young men are more likely to follow non-completers' study tracks, whereas young women are more likely to follow completers' study tracks. However, according to Table 5.2, this pattern does not necessarily hold true for Finland and Sweden when restricting the noncompleters' study tracks to cover only those with most, or all, years up to age 20 spent in full-time education. Instead, these two countries rather reveal a kind of segmentation by gender when it comes to noncompleters' study tracks: female non-completers seem to be more likely to follow "longer" (uninterrupted) study tracks, whereas male noncompleters tend to more often follow "shorter" or bumpier study tracks. Yet, despite this difference in post-compulsory-school engagement, their situation at age 21 is identical: still five years after compulsory school they only have an exam from primary education.

\begin{tabular}{|c|c|c|c|c|c|c|c|}
\hline \multirow{2}{*}{\multicolumn{2}{|c|}{$\begin{array}{l}\text { Years in post-compulsory } \\
\text { education up to age } 20 \\
\text { [stylized pathway] }\end{array}$}} & \multirow{3}{*}{$\begin{array}{r}\text { \%-share } \\
\text { in the } \\
\text { cluster } \\
35.0\end{array}$} & \multicolumn{2}{|c|}{ Gender } & \multicolumn{3}{|c|}{ Cohort of 16 -year-olds } \\
\hline & & & \multirow{2}{*}{$\begin{array}{r}\text { Male } \\
39.2\end{array}$} & \multirow{2}{*}{$\begin{array}{r}\text { Female } \\
29.9\end{array}$} & \multirow{2}{*}{$\begin{array}{l}1993 \\
32.6\end{array}$} & \multirow{2}{*}{$\begin{array}{r}1998 \\
38.0\end{array}$} & \multirow{2}{*}{$\begin{array}{r}2003 \\
34.5\end{array}$} \\
\hline All 5 years in & Denmark & & & & & & \\
\hline education & Finland & 21.6 & 19.7 & 24.4 & 20.6 & 21.0 & 23.3 \\
\hline \multirow[t]{2}{*}{ [11111] } & Norway & 30.3 & 31.3 & 28.9 & 25.2 & 31.4 & 33.8 \\
\hline & Sweden & 29.1 & 27.9 & 30.6 & 31.8 & 25.2 & 30.5 \\
\hline \multirow{4}{*}{$\begin{array}{l}\text { Delayed } \\
\text { start, all } 4 \\
\text { years in } \\
\text { education } \\
\text { [51111] }\end{array}$} & Denmark & 4.1 & 3.1 & 3.4 & 1.6 & 4.9 & 5.8 \\
\hline & Finland & 13.5 & 12.4 & 15.2 & 10.4 & 13.8 & 16.2 \\
\hline & Norway & 6.2 & 6.3 & 6.2 & 8.9 & 5.6 & 4.6 \\
\hline & Sweden & 7.7 & 8.0 & 7.4 & 12.3 & 8.8 & 3.4 \\
\hline \multirow{4}{*}{$\begin{array}{l}\text { Total share = } \\
{[11111]+} \\
{[51111]}\end{array}$} & Denmark & 39.1 & 42.3 & 33.3 & 34.2 & 42.9 & 40.3 \\
\hline & Finland & 35.1 & 32.1 & 39.6 & 31.0 & 34.8 & 39.5 \\
\hline & Norway & 36.5 & 37.6 & 35.1 & 34.1 & 37.0 & 38.4 \\
\hline & Sweden & 36.8 & 35.9 & 38.0 & 44.1 & 34.0 & 33.9 \\
\hline
\end{tabular}

Notes: See Figure 5.1 above. 
Also the time trend (across cohorts) for these two stylized educational pathways differs in certain respects from the one observed in Chapters 4 and 5. More precisely, while the previously reported results point to growing shares of also non-completers in study tracks, the pattern emerging from Table 5.2 is more diversified. In particular, this trend of increasingly more non-completers following study tracks does not seem to hold true for Swedish non-completers spending most, or all, of their time up to age 20 as full-time students. Instead, their share reveals a declining trend, especially with respect to delayed entries into postcompulsory education. Hence, the composition of Swedish noncompleters' study tracks seems to have undergone a major change in two, potentially closely linked dimensions: the shorter and more bumpy post-compulsory-school study tracks appear to have increased at the expense of longer and less interrupted study tracks and, as noted above, this tendency seems to have been stronger for young men than for young women. Indeed, this could well be one major mechanism underlying the growing gender gap in non-completion rates discernible for Sweden in Figure 2.2d of Chapter 2.

\subsubsection{Stylized employment pathways}

Next, we turn to the four stylized employment pathways defined in Table 5.1 above. As shown in that table, the only difference between the four pathways is the number of consecutive years spent by the noncompleter in post-compulsory education before moving into working life. Accordingly, these four pathways can be expected to detail the general picture mediated by the broad employment clusters underlying the results reported in Chapters 3 and 4 .

The first set of graphs in Figure 5.2 illustrates the outcome for the four Nordic countries under study when it comes to the stylized pathway where the young person continues directly in post-compulsory education for three consecutive years before moving into working life [11122]. The cross-country differences in non-completers' shares with respect to this pathway are rather small, ranging from $10-11 \%$ for Denmark and Norway to $14-15 \%$ for Finland and Sweden. Hence, many young people leave upper secondary school after three years for work, even though they have not succeeded in achieving an upper secondary certificate. Moreover, the role of these study-employment pathways has increased over time in all four countries, although this trend is less clearcut for Norway and Sweden (Table 5.3). 
The second set of graphs in Figure 5.2 gives the same information for the next stylized employment pathway: the person continues directly in post-compulsory education, but only for two years before substituting education with work [11222]. The share of non-completers allocated around this stylized pathway is lower (Finland) or much lower (Norway and Sweden), when compared to the corresponding share for the previous, more "standard" pathway [11122]. Only for Denmark do we get an opposite result. In all four countries, however, there has been a decline in the share of non-completers following this type of employmentinterrupted education pathway.

A slightly (Denmark) or notably lower (Finland, Norway, Sweden) share of the non-completers are clustered around the stylized employment pathway where non-completers spend only one year in full-time education straight after completing compulsory school before entering working life. In all four countries, many of these early employment tracks tend to be quite unstable, though. However, also these early employment pathways cover decreasing shares of the non-completers, when comparing the situation across cohorts. Again this holds true for all four Nordic countries.

The fourth and last set of graphs in Figure 5.2 concerns employment pathways typically starting with a year in inactivity straight after completing compulsory school before entering working life. The share of noncompleters following this type of track is comparatively large only in Finland. Moreover, this high share for Finnish non-completers reveals a weak, if any, downward trend over time. Among Danish non-completers, this type of pathway is clearly on the rise, with their share having rapidly approached the level observed for Finland (Table 5.3). In Norway and Sweden, the small share of non-completers allocated around this stylized pathway has declined further over time. 
Figure 5.2: Stylized employment pathways for non-completers, by country

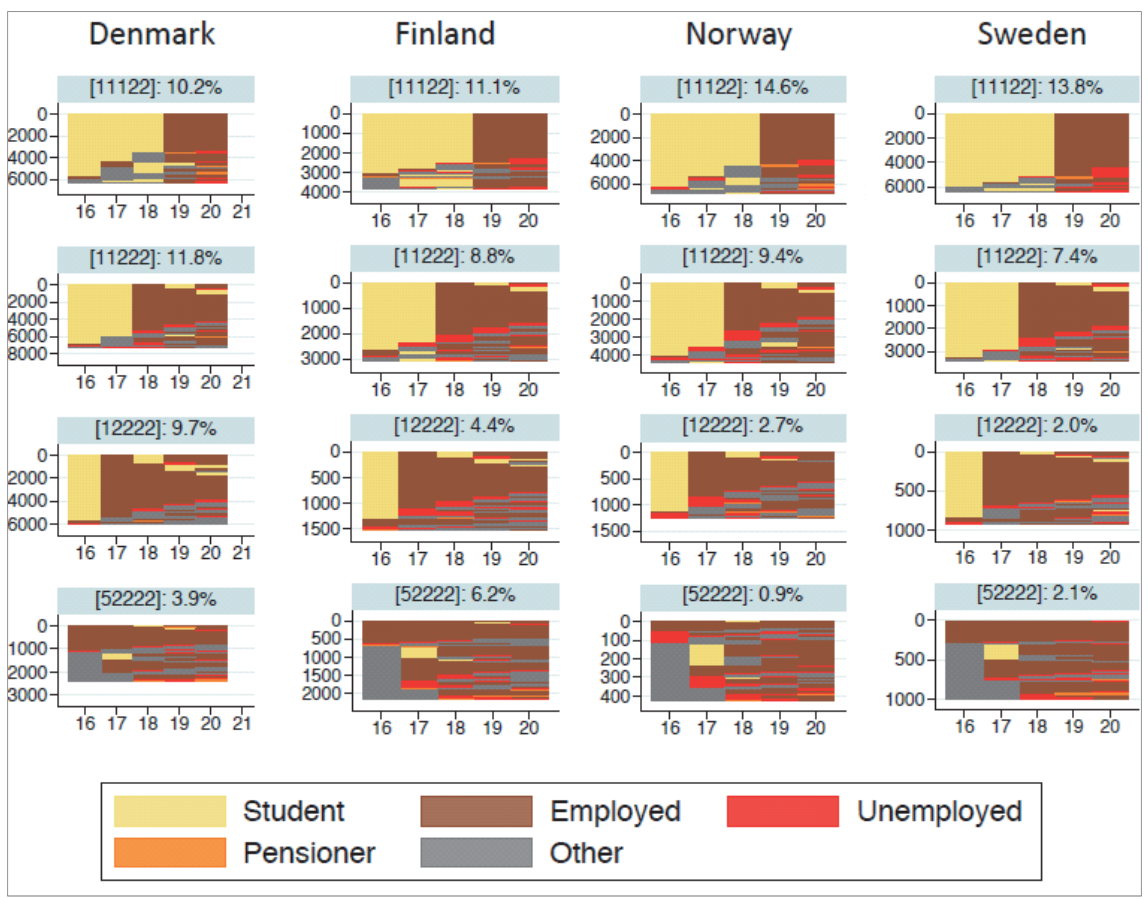

Notes: The clustering concerns individual school-to-work trajectories of non-completers when aged 16 to 20 , using clustering by reference sequences as described in sub-chapter 3.1. The vertical axis of each graph gives the absolute number of non-completers in the cluster, whereas the percentage share on top of each graph shows the relative share of non-completers in the cluster, by country. The country-specific percentage shares for the 16 stylized pathways thus sum up to $100 \%$ for each country. The number in bracketed parentheses on top of each graph refers to the stylized pathway in question as described in Table 5.1. For more details, by gender and cohort, see Table 5.3 below.

All in all, two main contentions can be made based on these four stylized employment pathways. First, employment tracks are more prevalent among Danish non-completers than among non-completers in the other three countries: almost $36 \%$ of the Danish non-completers go into one of the four employment pathways. Second, the role of these employment pathways, as measured by the share of non-completers covered, increases with the number of years spent in full-time education before moving into working life. Put differently, the fewer years non-completers initially spend in post-compulsory education, the less likely they are to move into employment careers of a more permanent nature. Both findings lend further support to the conclusions drawn in Chapters 3 and 4. 
We conclude this section with a few words on the distribution of young men and women across these four stylized employment pathways. Based on Table 5.3, there seems to be no obvious pattern in this respect, neither within countries nor between countries. The [11122] pathway covers a larger share of young men than of young women, except in Denmark where the situation is the opposite. The [11222] pathway covers a larger share of young men than of young women, except in Denmark and Sweden where the situation is the opposite. The [12222] pathway covers a larger share of young men than of young women, except in Norway and Sweden where the gendered shares are approximately of the same size. The [52222] pathway, finally, covers a larger share of young men than of young women, except in Norway where these two shares are, again, of much the same size.

Table 5.3: Distribution (\%-share) of young non-completers across the four stylized employment pathways displayed in Figure 5.2, by country, gender and cohort

\begin{tabular}{llrrrrrr}
$\begin{array}{l}\text { Years in post-compulsory education } \\
\text { before moving into working life [stylized } \\
\text { pathways] }\end{array}$ & $\begin{array}{r}\text { \%-share } \\
\text { in the } \\
\text { cluster }\end{array}$ & Male & Female & 1993 & 1998 & $\mathbf{2 0 0 3}$ \\
first 3 years in education & Denmark & 10.2 & 9.0 & 9.6 & 8.6 & 9.8 & 12.1 \\
[11122] & Finland & 11.1 & 13.3 & 7.8 & 7.7 & 11.4 & 13.8 \\
& Norway & 14.6 & 15.1 & 13.8 & 11.6 & 16.8 & 15.1 \\
& Sweden & 13.8 & 14.3 & 13.2 & 8.3 & 18.3 & 13.9 \\
first 2 years in education & Denmark & 11.8 & 12.1 & 12.4 & 14.5 & 10.0 & 11.0 \\
[11222] & Finland & 8.8 & 9.9 & 7.2 & 9.8 & 9.7 & 6.9 \\
& Norway & 9.4 & 10.1 & 8.5 & 10.3 & 10.7 & 7.6 \\
& Sweden & 7.4 & 6.8 & 8.2 & 6.8 & 9.2 & 6.2 \\
first (initial) year in educa- & Denmark & 9.7 & 11.0 & 9.8 & 12.0 & 9.0 & 8.4 \\
tion & Finland & 4.4 & 4.7 & 3.8 & 5.5 & 4.7 & 2.9 \\
[12222] & Norway & 2.7 & 2.7 & 2.8 & 3.3 & 3.3 & 1.6 \\
& Sweden & 2.0 & 1.9 & 2.1 & 2.3 & 2.2 & 1.6 \\
No entry into post- & Denmark & 3.9 & 3.9 & 2.8 & 3.3 & 3.6 & 4.7 \\
compulsory education & Finland & 6.2 & 6.8 & 5.4 & 5.9 & 7.3 & 5.2 \\
[52222] & Norway & 0.9 & 0.8 & 1.1 & 1.4 & 1.0 & 0.4 \\
& Sweden & 2.1 & 2.5 & 1.7 & 2.4 & 2.3 & 1.8 \\
Total share = & Denmark & 35.6 & 36.0 & 34.6 & 38.4 & 32.4 & 36.2 \\
[11122] + [11222] + & Finland & 30.5 & 34.7 & 24.2 & 28.9 & 33.1 & 28.8 \\
[12222] + [5222] & Norway & 27.6 & 28.7 & 26.2 & 26.6 & 31.8 & 24.7 \\
& Sweden & 25.3 & 25.5 & 25.2 & 19.8 & 32.0 & 23.5 \\
\hline
\end{tabular}

Notes: See Figure 5.2 above.

\subsubsection{Stylized unemployment pathways}

In Table 5.1, we distinguish between three stylized unemployment pathways, which differ from each other only with respect to the number of years spent by the young non-completer in post-compulsory education before becoming unemployed (i.e., registering as an unemployed jobseeker). The first set of graphs in Figure 5.3 departs from the typical 
upper secondary study track of three years, upon which follows spells in unemployment [11133]. The second set of graphs highlights a situation where the young non-completer drops out from post-compulsory education already after two years [11333], whereas the last set of graphs covers a situation with the young non-completer moving into unemployment already after one year in post-compulsory education [13333].

In line with the results reported in Chapters 3 and 4, these stylized unemployment pathways are relatively infrequent among young Nordic noncompleters: in all four countries, each of them covers, at most, some $5 \%$ of the non-completers - typically much less. The only exception is Sweden with a share of close to $12 \%$ of the non-completers moving into unemployment upon leaving upper secondary school after three years (without a degree). The corresponding share is much lower in the other three countries, with Denmark down at $2.1 \%$. A common feature of the four countries, however, is that the share of non-completers following this type of dropout-unemployment trajectory reveals a weak decline across cohorts (Table 5.4). Another joint feature is a minor difference in the shares of young men and young women following this type of trajectory.

Figure 5.3: Stylized unemployment pathways of non-completers, by country

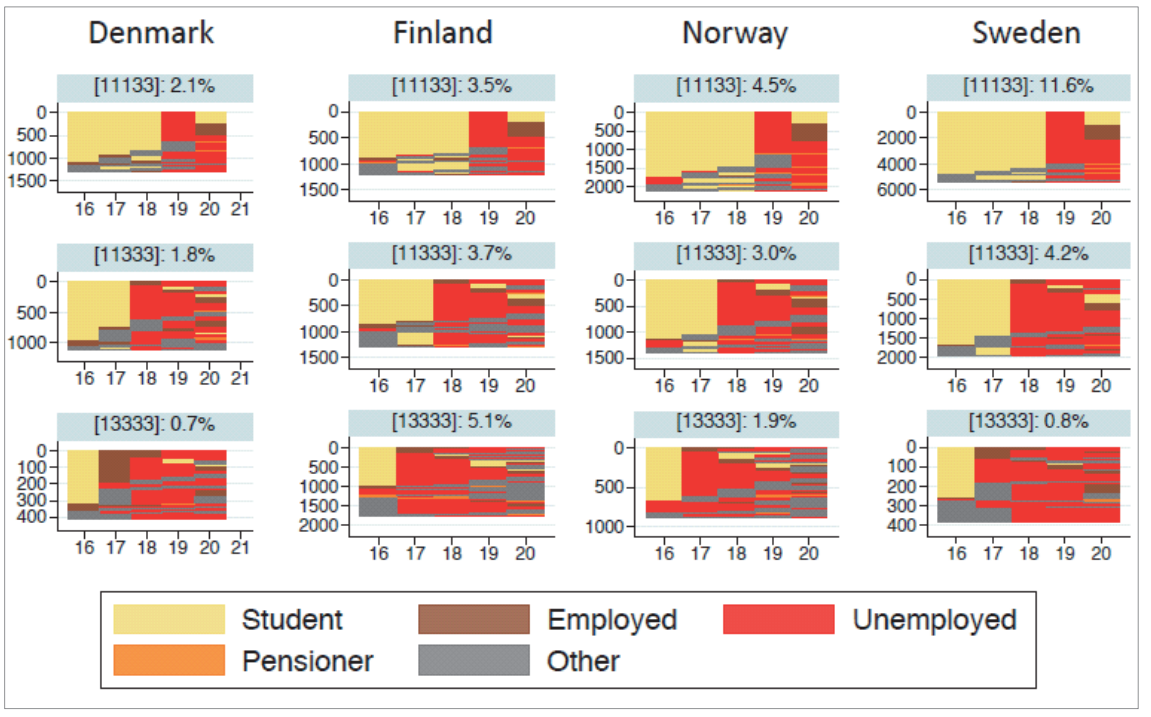

Notes: The clustering concerns individual school-to-work trajectories of non-completers when aged 16 to 20 , using clustering by reference sequences as described in sub-chapter 3.1. The vertical axis of each graph gives the absolute number of non-completers in the cluster, whereas the percentage share on top of each graph shows the relative share of non-completers in the cluster, by country. The country-specific percentage shares for the 16 stylized pathways thus sum up to $100 \%$ for each country. The number in bracketed parentheses on top of each graph refers to the stylized pathway in question as described in Table 5.1. For more details, by gender and cohort, see Table 5.4 below. 
Compared to the [11133] pathway, Sweden has a notably lower share of non-completers ending up in unemployment when dropping out from post-compulsory education already after two years. This holds true also for Norway, whereas these two unemployment pathways cover approximately equal shares of the non-completers in both Denmark and Finland. However, the ranking of the four countries remains unchanged with Sweden showing up with the highest and Denmark with the lowest share. A distinct feature of this [11333] pathway is that the share of young non-completers following this type of school-dropout trajectory has declined substantially over time. This decline has been particularly strong for Finland. Additionally, Finland is characterised by a clearly higher share of young male than of young female non-completers following this type of dropout-unemployment track. However, all these peculiarities are related to the difficult employment situation of especially low-educated youngsters following upon the deep recession that the Finnish economy plunged into in the early 1990s (cf. the Finnish cluster results for non-completers in Chapter 3). The younger Finnish cohorts, in contrast, face an unemployment situation which is quite similar to that experienced by other young Nordic non-completers.

Also the share of non-completers showing up as unemployed jobseekers already when aged 17, is conspicuously high for Finland (about 5\%), but very low in the other three countries. The explanation is, by and large, the same as in the previous case: widespread unemployment among low-educated young people starting in the deep recession years of the early 1990s. As is evident in Table 5.4, the share of Finnish non-completers moving into unemployment more or less straight after compulsory school has shrunk from about $13 \%$ in the oldest (1993) cohort to $1.2 \%$ in the youngest (2003) cohort, a share which is very much in line with the shares observed for the other three countries. Finally, as for the other two stylized unemployment pathways, we see declining shares across cohorts in all four countries and minor, if any, differences in the shares of the two genders. 


\begin{tabular}{|c|c|c|c|c|c|c|c|}
\hline \multirow{2}{*}{\multicolumn{2}{|c|}{$\begin{array}{l}\text { Years in post-compulsory education } \\
\text { before moving into unemployment } \\
\text { [stylized pathway] }\end{array}$}} & \multirow{3}{*}{$\begin{array}{r}\text { \%-share } \\
\text { in the } \\
\text { cluster } \\
2.1\end{array}$} & \multicolumn{2}{|c|}{ Gender } & \multicolumn{3}{|c|}{ Cohort of 16 -year-olds } \\
\hline & & & \multirow{2}{*}{$\begin{array}{r}\text { Male } \\
2.0\end{array}$} & \multirow{2}{*}{$\begin{array}{r}\text { Female } \\
2.7\end{array}$} & \multirow{2}{*}{$\begin{array}{r}1993 \\
2.3\end{array}$} & \multirow{2}{*}{$\begin{array}{r}1998 \\
2.4\end{array}$} & \multirow{2}{*}{$\begin{array}{r}2003 \\
1.6\end{array}$} \\
\hline First 3 years in education & Denmark & & & & & & \\
\hline \multirow[t]{3}{*}{ [11133] } & Finland & 3.5 & 3.7 & 3.1 & 4.2 & 3.5 & 2.8 \\
\hline & Norway & 4.5 & 4.8 & 4.1 & 5.4 & 4.2 & 4.1 \\
\hline & Sweden & 11.6 & 11.8 & 11.2 & 12.6 & 10.7 & 11.5 \\
\hline \multirow{4}{*}{$\begin{array}{l}\text { First } 2 \text { years in education } \\
\text { [11333] }\end{array}$} & Denmark & 1.8 & 1.6 & 2.3 & 2.0 & 1.9 & 1.6 \\
\hline & Finland & 3.7 & 4.2 & 2.9 & 7.1 & 2.6 & 1.7 \\
\hline & Norway & 3.0 & 3.3 & 2.6 & 4.7 & 2.5 & 2.0 \\
\hline & Sweden & 4.2 & 4.1 & 4.4 & 6.1 & 3.4 & 3.6 \\
\hline \multirow{4}{*}{$\begin{array}{l}\text { First (initial) year in } \\
\text { education } \\
\text { [13333] }\end{array}$} & Denmark & 0.7 & 0.7 & 0.9 & 0.8 & 0.7 & 0.5 \\
\hline & Finland & 5.1 & 5.4 & 4.6 & 12.8 & 2.2 & 1.2 \\
\hline & Norway & 1.9 & 2.0 & 1.9 & 3.5 & 1.3 & 1.1 \\
\hline & Sweden & 0.8 & 0.8 & 0.9 & 1.3 & 0.6 & 0.6 \\
\hline \multirow{4}{*}{$\begin{array}{l}\text { Total share }= \\
{[11133]+[11333]+} \\
{[13333]}\end{array}$} & Denmark & 4.6 & 4.3 & 5.9 & 5.1 & 5.0 & 3.7 \\
\hline & Finland & 12.3 & 13.3 & 10.6 & 24.1 & 8.3 & 5.7 \\
\hline & Norway & 9.4 & 10.1 & 8.6 & 13.6 & 8.0 & 7.2 \\
\hline & Sweden & 16.6 & 16.7 & 16.5 & 20.0 & 14.7 & 15.7 \\
\hline
\end{tabular}

Notes: See Figure 5.3 above.

Broadly speaking, these stylized unemployment pathways thus cover relatively small and further declining shares of young Nordic noncompleters. While this is the outcome of a declining trend observed for all three pathways, the early dropout trajectories [13333 and 11333] have contributed more to this decline than the more "standard" [11133] trajectory. This finding is likely to be the outcome of several trends. This decline in the share of young non-completers following dropoutunemployment trajectories may well reflect a situation where young people truly try to avoid this type of high-risk track. However, the decline may also simply reflect a situation where the tightened conditions for receiving unemployment benefits have made young people less inclined to register as unemployed jobseekers (in which case they go into the dumping category labelled "other"). The observed decline is also likely to reflect intensified youth unemployment policies, coupled with a favourable economic development, where low-educated youngsters registering as unemployed have increasingly been directed either back into school or into various types of arrangements equipping them with work practises (in the former case they turn up in our datasets as fulltime students, in the latter case as employed). Indeed, the time trends for the stylized educational and employment pathways explored in the previous sections seem to lend further support to this last interpretation. The much higher shares of young non-completers covered by the [11133] pathway may, in turn, be taken to indicate that non-completers having spent more years in post-compulsory education before experi- 
encing (registered) unemployment are, first and foremost, slightly older and, hence, more likely to start in "ordinary" active labour market programs when facing prolonged difficulties in finding a job.

With both Finland, Norway and Sweden fitting into this overall picture, Denmark is an profound exception with small and further declining shares of young non-completers moving early into unemploymentdominated pathways. Even taken together, the three stylized unemployment pathways cover less than $4 \%$ of the Danish non-completers in the youngest (2003) cohort (Table 5.4). The corresponding share is $5.7 \%$ for Finland, $7.1 \%$ for Norway and as high as $15.7 \%$ for Sweden.

\subsubsection{Stylized disability-benefit (pensioner) pathways}

The three stylized disability-benefit pathways describe a situation where the young non-completer moves into disability arrangements after three, two or only one, if any, years in post-compulsory education [11144, 11444, 14444]. Depending on the system in place, the young person is either on disability benefits of a more permanent nature, or on so-called rehabilitation benefits, which are typically meant to be temporary but may also precede more permanent disability arrangements.

Figure 5.4 contains these three sets of stylized disability-benefit pathways, showing the prevalence of each of them in the four Nordic countries under study. A general observation is that few young noncompleters move into this type of post-compulsory-school track. Taken together, the three graphs illustrating the situation for Denmark cover an average of only $1.3 \%$ of Danish non-completers. The three graphs also reflect the country's institutional setting with young people being granted a disability pension only from age 18 (cf. Chapter 2). In view of this, it is not surprising that a majority of young Danish non-completers move into disability pension arrangements immediately when fulfilling the age condition. This is also the category of young disability pensioners that stands behind the observed increase across cohorts in the Danish share of disability-pension-receiving non-completers (Table 5.5). 
Figure 5.4: Stylized disability-benefit pathways of non-completers, by country

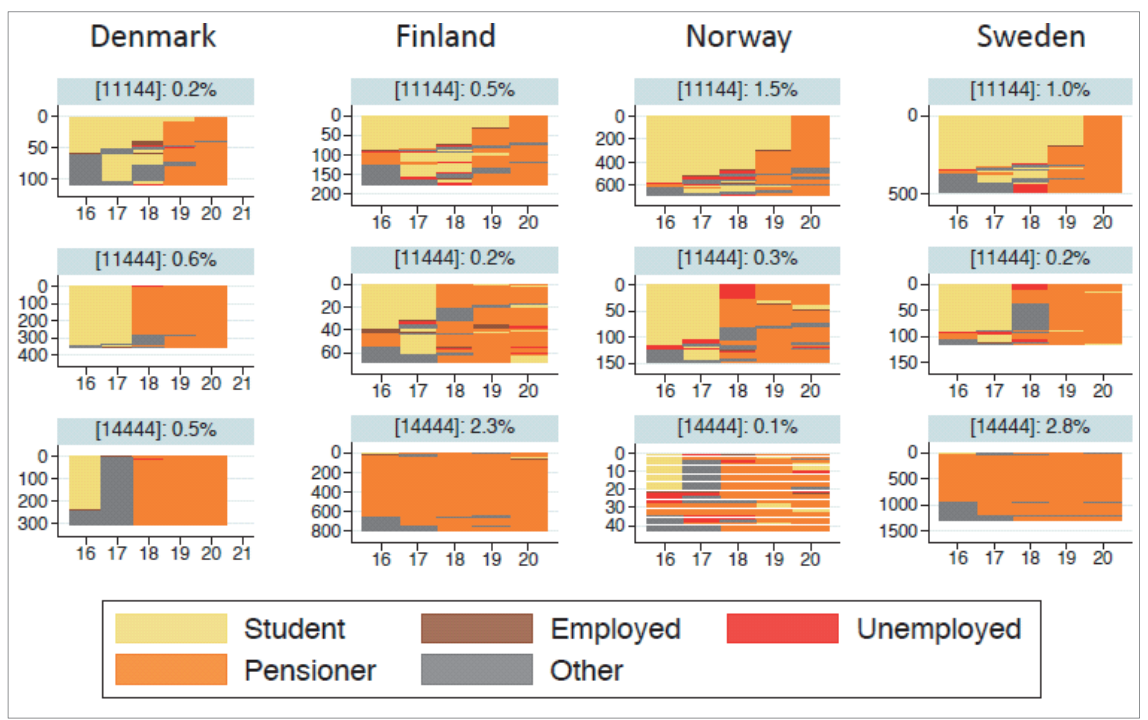

Notes: The clustering concerns individual school-to-work trajectories of non-completers when aged 16 to 20 , using clustering by reference sequences as described in sub-chapter 3.1. The vertical axis of each graph gives the absolute number of non-completers in the cluster, whereas the percentage share on top of each graph shows the relative share of non-completers in the cluster, by country. The country-specific percentage shares for the 16 stylized pathways thus sum up to $100 \%$ for each country. The number in bracketed parentheses on top of each graph refers to the stylized pathway in question as described in Table 5.1. For more details, by gender and cohort, see Table 5.5 below.

Also Norway displays an increasing trend in the share of young noncompleters going into disability arrangements. A growing share of Norwegian youth receiving temporary or permanent health-related disability benefits is, in effect, also reported by, for example, Bragstad and Brage (2011), with this development shown to be mainly due to a steep rise in young disability beneficiaries suffering from severe mental diseases. Otherwise, the situation in Norway looks quite different compared to that observed for Denmark. In particular, most Norwegian noncompleters following a disability-benefit track have stayed in postcompulsory education for three or more years before showing up as a disability beneficiary [11144]. This is also the category having increased over time, especially among non-completers in the youngest (2003) cohort (Table 5.5). Most probably, the main reason for the growth in the number of young disability beneficiaries being concentrated to this particular track is the institutional changes made in Norway with respect to young peoples' possibilities to move into disability arrangements at an early age (cf. Chapter 2). 
Another pattern emerges for Finland and Sweden. Both countries appear to have comparatively large shares of young non-completers going into disability arrangements straight after completing compulsory school. This type of disability benefit track is totally missing in Denmark and covers a negligible share of young non-completers in Norway. However, the share of Finnish and Swedish youngsters following such trajectories has declined remarkably over cohorts, mainly due to profound changes in the disability benefit system for young people (cf. Chapter 2). Indeed, the change has been quite dramatic for Sweden: a share of $0.1 \%$ in the youngest (2003) cohort compared to about $4.5 \%$ in the two older cohorts (Table 5.5). Simultaneously, there has been a notable increase, from $0.4 \%$ to $1.9 \%$, in the share of young Swedish non-completers moving into disability arrangements at a slightly higher age, viz. after the age of 18. While we observe a similar pattern for Finland, the changes in shares across cohorts have been much smaller.

\begin{tabular}{|c|c|c|c|c|c|c|c|}
\hline \multirow{2}{*}{\multicolumn{2}{|c|}{$\begin{array}{l}\text { Years in post-compulsory education before } \\
\text { moving into disability arrangements } \\
\text { [stylized pathway] }\end{array}$}} & \multirow{3}{*}{$\begin{array}{r}\text { \%-share } \\
\text { in the } \\
\text { cluster } \\
0.2\end{array}$} & \multicolumn{2}{|c|}{ Gender } & \multicolumn{3}{|c|}{ Cohort of 16-year-olds } \\
\hline & & & \multirow{2}{*}{$\begin{array}{r}\text { Male } \\
0.2\end{array}$} & \multirow{2}{*}{$\begin{array}{r}\text { Female } \\
0.2\end{array}$} & \multirow{2}{*}{$\begin{array}{r}1993 \\
0.2\end{array}$} & \multirow{2}{*}{$\begin{array}{r}1998 \\
0.2\end{array}$} & \multirow{2}{*}{$\begin{array}{r}2003 \\
0.2\end{array}$} \\
\hline First 3 years in education & Denmark & & & & & & \\
\hline \multirow[t]{3}{*}{ [11144] } & Finland & 0.5 & 0.4 & 0.6 & 0.4 & 0.5 & 0.6 \\
\hline & Norway & 1.5 & 1.4 & 1.6 & 1.5 & 1.0 & 1.8 \\
\hline & Sweden & 1.0 & 1.1 & 1.0 & 0.4 & 0.7 & 1.9 \\
\hline \multirow{4}{*}{$\begin{array}{l}\text { First } 2 \text { years in education } \\
{[11444]}\end{array}$} & Denmark & 0.6 & 0.5 & 0.5 & 0.5 & 0.3 & 0.7 \\
\hline & Finland & 0.2 & 0.2 & 0.2 & 0.1 & 0.3 & 0.2 \\
\hline & Norway & 0.3 & 0.3 & 0.4 & 0.3 & 0.3 & 0.4 \\
\hline & Sweden & 0.2 & 0.3 & 0.3 & 0.1 & 0.2 & 0.3 \\
\hline \multirow{4}{*}{$\begin{array}{l}\text { First (initial) year in education } \\
\text { [14444] }\end{array}$} & Denmark & 0.5 & 0.4 & 0.3 & 0.3 & 0.5 & 0.7 \\
\hline & Finland & 2.3 & 2.2 & 2.3 & 2.6 & 2.6 & 1.5 \\
\hline & Norway & 0.1 & 0.1 & 0.1 & 0.1 & 0.1 & 0.1 \\
\hline & Sweden & 2.8 & 3.0 & 2.6 & 4.4 & 4.5 & 0.1 \\
\hline \multirow{4}{*}{$\begin{array}{l}\text { Total share }= \\
{[11144]+[11444]+} \\
{[14444]}\end{array}$} & Denmark & 1.3 & 1.1 & 1.0 & 1.0 & 1.0 & 1.6 \\
\hline & Finland & 3.0 & 2.8 & 3.1 & 3.1 & 3.4 & 2.3 \\
\hline & Norway & 1.9 & 1.8 & 2.1 & 1.9 & 1.4 & 2.3 \\
\hline & Sweden & 4.0 & 4.4 & 3.9 & 4.9 & 5.4 & 2.3 \\
\hline
\end{tabular}

Notes: See Figure 5.4 above.

In sum, for all four Nordic countries we observe relatively few noncompleters entering disability arrangements at an early age. Another common feature of the four countries is that the institutional setting in place is strongly reflected in the type of disability-benefit trajectories followed by a majority of the country's young people showing up, sooner or later, as disability beneficiaries. Likewise, the institutional changes made during the time period covered by our three youth cohorts are clearly mirrored by the cross-cohort changes seen in the relative im- 
portance of different types of tracks ending up in some form of disability arrangements. In particular, the overall impression is that these institutional changes have, first and foremost, only delayed these youngsters' possibilities to enter disability arrangements, until they fulfil the new (increased) age condition. Finally, a third common feature of the four countries is that there are no striking gender differences in the disability pathways in any of the countries.

\subsubsection{Stylized inactivity ("other") pathways}

Finally we turn the focus to the clusters of young non-completers built around the four stylized inactivity pathways defined in Table 5.1. As often pointed out earlier, the category of other inactivity embraces all young people not appearing in any of the large administrative registers from which our datasets are compiled. The allocation of non-completers across these four stylized pathways is displayed in Figure 5.5, separately for each country. Again, these stylized pathways provide, at best, a much more detailed description of young non-completers' early inactivitydominated pathways than the more broadly defined inactivity clusters of Chapters 3 and 4.

In all four countries, we see large shares of young non-completers continuing directly in post-compulsory education for three or more years before dropping out from school (without a degree) just to withdraw also from the labour market, into inactivity. This share of young non-completers is particularly high in Norway (about 13\%). The corresponding share in the other three countries is only about half this size, but still some 6 to 7\%. Moreover, this type of dropout-inactivity trajectory covers increasing shares of young non-completers. The increase over cohorts has been remarkably strong among Norwegian and Swedish non-completers, with a growth from 11 to $17 \%$ in Norway and from 3 to $9 \%$ in Sweden (Table 5.6.). While an increasing trend is discernible also for Finland, it is notably weaker (less than two percentage points). In Denmark, in contrast, the share of young non-completers in such tracks has declined over time. 
Figure 5.5: Stylized inactivity ("other") pathways of non-completers, by country

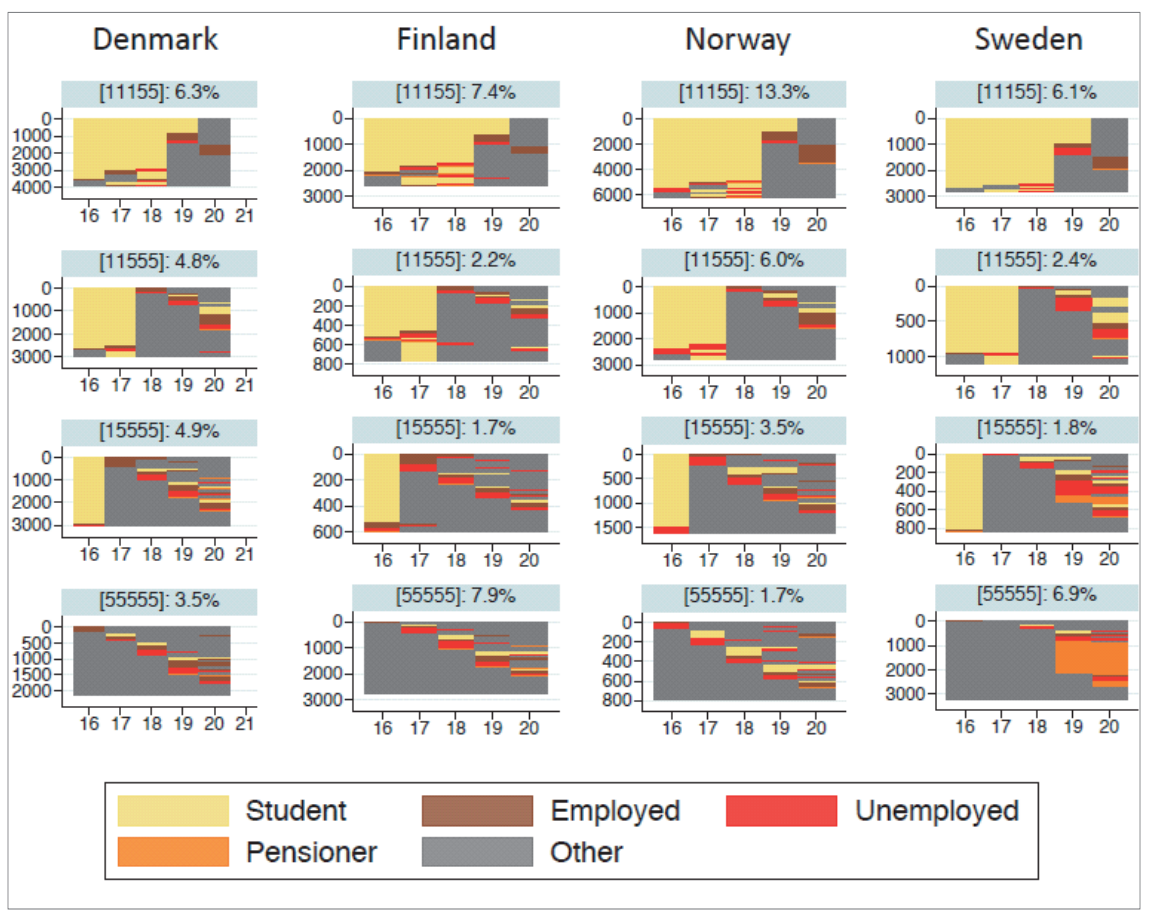

Notes: The clustering concerns individual school-to-work trajectories of non-completers when aged 16 to 20 , using clustering by reference sequences as described in sub-chapter 3.1. The vertical axis of each graph gives the absolute number of non-completers in the cluster, whereas the percentage share on top of each graph shows the relative share of non-completers in the cluster, by country. The country-specific percentage shares for the 16 stylized pathways thus sum up to $100 \%$ for each country. The number in bracketed parentheses on top of each graph refers to the stylized pathway in question as described in Table 5.1. For more details, by gender and cohort, see Table 5.6 below.

Also early dropout into inactivity - already after two or only one year in post-compulsory education - turns out to be a comparatively common phenomenon, but mainly in Denmark and Norway. Taken together, these two inactivity pathways cover, in both countries, almost $10 \%$ of the young non-completers. Indeed, when further adding the share of noncompleters allocated around the previous [11155] pathway, we cover $16 \%$ of the Danish non-completers and as much as $23 \%$ of the Norwegian non-completers. However, for both countries we observe a declining trend in the shares of young non-completers following these types of early-dropout-inactivity tracks. The corresponding share for Finland and Sweden is much lower, covering only about $4 \%$ of the noncompleters. But in both countries, these shares are increasing rather than decreasing.

The situation is reversed with respect to inactivity tracks starting straight after completion of compulsory school [55555] in the sense that 
these early inactivity tracks are quite prevalent among Finnish and Swedish non-completers. Moreover, both countries have seen a doubling across cohorts in the share of non-completers in such tracks: from less than $5 \%$ in the oldest (1993) cohort to nearly $11 \%$ or more in the youngest (2003) cohort. The corresponding share is much lower in Denmark but increasing, whereas it is very low in Norway and decreasing.

\begin{tabular}{|c|c|c|c|c|c|c|c|}
\hline \multirow{2}{*}{\multicolumn{2}{|c|}{$\begin{array}{l}\text { Years in post-compulsory education before } \\
\text { moving into inactivity ('other') } \\
\text { [stylized pathway] }\end{array}$}} & \multirow{3}{*}{$\begin{array}{r}\text { \%-share } \\
\text { in the } \\
\text { cluster } \\
6.3\end{array}$} & \multicolumn{2}{|c|}{ Gender } & \multicolumn{3}{|c|}{ Cohort of 16 -year-olds } \\
\hline & & & Male & Female & 1993 & 1998 & 2003 \\
\hline \multirow{4}{*}{$\begin{array}{l}\text { First } 3 \text { years in education } \\
\text { [11155] }\end{array}$} & Denmark & & 5.9 & 8.0 & 6.7 & 6.9 & 5.5 \\
\hline & Finland & 7.4 & 7.1 & 7.8 & 6.6 & 7.2 & 8.3 \\
\hline & Norway & 13.3 & 12.3 & 14.8 & 10.6 & 11.9 & 17.0 \\
\hline & Sweden & 6.1 & 6.3 & 5.9 & 2.9 & 5.8 & 8.8 \\
\hline \multirow{4}{*}{$\begin{array}{l}\text { First } 2 \text { years in education } \\
\text { [11555] }\end{array}$} & Denmark & 4.8 & 4.0 & 6.9 & 6.7 & 3.8 & 4.0 \\
\hline & Finland & 2.2 & 1.8 & 2.7 & 0.8 & 2.8 & 2.8 \\
\hline & Norway & 6.0 & 5.3 & 6.8 & 6.2 & 5.2 & 6.5 \\
\hline & Sweden & 2.4 & 2.4 & 2.4 & 2.4 & 2.2 & 2.5 \\
\hline \multirow{4}{*}{$\begin{array}{l}\text { First (initial) year in education } \\
\text { [15555] }\end{array}$} & Denmark & 4.9 & 3.7 & 6.9 & 5.5 & 4.6 & 4.6 \\
\hline & Finland & 1.7 & 1.3 & 2.3 & 0.5 & 2.4 & 2.0 \\
\hline & Norway & 3.5 & 3.0 & 4.2 & 4.4 & 3.2 & 2.9 \\
\hline & Sweden & 1.8 & 1.7 & 1.9 & 1.7 & 1.6 & 2.1 \\
\hline \multirow{4}{*}{$\begin{array}{l}\text { No entry into post-compulsory } \\
\text { education } \\
\text { [55555] }\end{array}$} & Denmark & 3.5 & 2.7 & 3.6 & 2.8 & 3.4 & 4.1 \\
\hline & Finland & 7.9 & 6.6 & 9.7 & 4.9 & 8.0 & 10.6 \\
\hline & Norway & 1.8 & 1.2 & 2.2 & 2.6 & 1.5 & 1.0 \\
\hline & Sweden & 6.9 & 7.4 & 6.3 & 4.3 & 4.2 & 11.3 \\
\hline \multirow{4}{*}{$\begin{array}{l}\text { Total share }= \\
{[11155]+[11555]+} \\
{[15555]+[55555]}\end{array}$} & Denmark & 19.5 & 16.3 & 25.4 & 21.7 & 18.7 & 18.2 \\
\hline & Finland & 19.2 & 16.8 & 22.5 & 12.8 & 20.4 & 23.7 \\
\hline & Norway & 24.6 & 21.8 & 28.0 & 23.8 & 21.8 & 27.4 \\
\hline & Sweden & 17.2 & 17.8 & 16.5 & 11.3 & 13.8 & 24.7 \\
\hline
\end{tabular}

Notes: See Figure 5.5 above.

All in all, the share of young non-completers following inactivitydominated tracks at an early age is strikingly high in all four countries. Moreover, increasingly larger shares of the non-completers show up in such tracks, except in Denmark (Table 5.6). This increase has been quite moderate in Norway, but with the initial level being remarkably high when compared to the other three countries: from a share of $22-23 \%$ in the older cohorts to a share exceeding $27 \%$ in the youngest (2003) cohort. The development observed for Finland and Sweden can rather be described as explosive with an increase from $13 \%$ to almost $24 \%$ among Finnish non-completers and from $11 \%$ to almost 25\% among Swedish non-completers. However, a look behind these average shares reveals distinct differences in the dropout-inactivity tracks actually dominating in each country and the time trends emerging for these various tracks. Indeed, it is tempting to explain these differences in country-specific pat- 
terns with the underlying institutional setting and the restrictions made over the past decades especially with respect to the eligibility conditions for young people concerning unemployment and disability benefits. Finally, it may be noted that young women tend to be more likely to follow early inactivity-dominated tracks, when compared to their male peers. Only in Sweden do young men seem to face an equally high risk or an even slightly higher risk of ending up in such tracks.

\subsubsection{Main findings}

The construction of a total of 16 stylized pathways and the results obtained for these pathways sharpen further the picture formed so far of young Nordic non-completers' early post-compulsory-school experiences, up to age 20. While there are lots of distinct differences between the four Nordic countries in this respect, we can also observe important similarities. All these details, including results by gender and cohort, have been presented and discussed above. In sum, these results show the following.

The two stylized educational pathways confirm the overall picture for young Nordic non-completers painted by the results presented in previous chapters, notably in Chapters 4 and 5. In particular, large shares of also non-completers continue, either directly or after a break year, in post-compulsory education. Indeed, remarkably many of them spend all or most of their time, from age 16 up to age 20, as full-time students, but without completing an upper secondary degree within five years after leaving compulsory school.

The four stylized employment pathways show, once again, that employment tracks are more prevalent among Danish non-completers than among non-completers in the other three countries. Nonetheless, the four countries share the feature of the role of these employment pathways, as measured by the share of non-completers covered, increasing with the number of years spent in full-time education before moving into working life (but without having completed an upper secondary degree). In other words, the earlier the non-completers leave postcompulsory education, the less likely they are to move into employment careers of a more permanent nature.

Compared to the stylized educational and employment pathways, the stylized unemployment pathways cover relatively small shares of young Nordic non-completers. This is well in line with the observations made in the previous chapters. Moreover, the share of young non-completers in registered unemployment has declined notably over time. This declin- 
ing trend across cohorts is discernible for all three stylized unemployment pathways. It is worth noting, though, that the early- dropoutunemployment trajectories have contributed more to this decline than the more "standard" trajectory characterised by unemployment spells following upon dropout only after three years in upper secondary education, without completion of a degree. As discussed above, this outcome obviously mirrors the combined effect of several circumstances.

When it comes to other types of NEET activities, our results indicate the following. The institutional setting in place strongly affects the type of disability-benefit trajectories followed by a majority of the country's young people showing up, sooner or later, as disability beneficiaries. Likewise, major institutional reforms undertaken since the early 1990s are clearly reflected in the changing relative importance of different types of tracks ending up in some form of disability arrangements. Indeed, the overwhelming impression is that these institutional changes have merely delayed young non-completers' possibilities to enter disability arrangements. Obviously, this also explains why we in all four Nordic countries observe relatively few non-completers entering disability arrangements at an early age. In all four countries, the NEET activities of non-completers are dominated by withdrawal from both education and working life already at an early age. Another distinct feature is that relatively more young women than young men go into this type of high-risk track straight or almost straight after having left compulsory school. Only in Sweden do young men seem to face an equally high risk or an even slightly higher risk of ending up in such tracks.

Another way of summarising these main findings is to rely on two more figures which highlight the observed cross-country similarities and dissimilarities from slightly different angles. The first figure, Figure 5.6, summarises the distribution of non-completers according to the number of initial years (none, one, two, three, or more than three) spent by the non-completers in post-compulsory education before dropping out without having achieved an upper secondary certificate. The second figure, Figure 5.7, is organised in a reversed manner: it departs from the distribution of young non-completers across the five "end-up" activities without account being made for the number of initial years spent in post-compulsory education before entering the activity in question. Hence, Figure 5.6 is built on criterion 1 and Figure 5.7 on criterion 2 of Table 5.1. Apart from these two figures highlighting our main findings for the group of non-completers as such, we also relate these noncompleter-specific outcomes to the full youth population. As in the previous chapter, this is done in order to provide a fuller picture of the rela- 
tive importance of different non-completer tracks in the four Nordic countries under study.

Figure 5.6 illustrates well a finding pointed out earlier in this report: large shares of Nordic non-completers spend most of their postcompulsory years, up to age 20 , as full-time students, but without completing an upper secondary degree by age 21 . As shown in the figure, between 35\% (Finland) and 39\% (Denmark) of young non-completers spend more than three years in upper secondary education before dropping out temporarily or permanently. Also the share of young noncompleters continuing for exactly three years in upper secondary education before dropping out is relatively high, especially in Norway and Sweden. This implies that about $70 \%$ of both Norwegian and Swedish noncompleters spend three or more years in upper secondary education, but without achieving a degree. The corresponding share is notably lower (close to 58\%) for Danish and Finnish non-completers. This is mainly due to Danish and Finnish non-completers being almost twice as likely as Norwegian and Swedish non-completers to drop out already after one year in post-compulsory education. Finnish non-completers also have the highest probability of following a non-starter pathway: about $14 \%$ of Finnish non-completers do not continue in post-compulsory education. This share is strikingly low (under $3 \%$ ) for Norway.

Figure 5.6: Distribution (\%-share) of non-completers across stylized pathways by number of initial years spent in post-compulsory education before dropping out, for the four Nordic countries

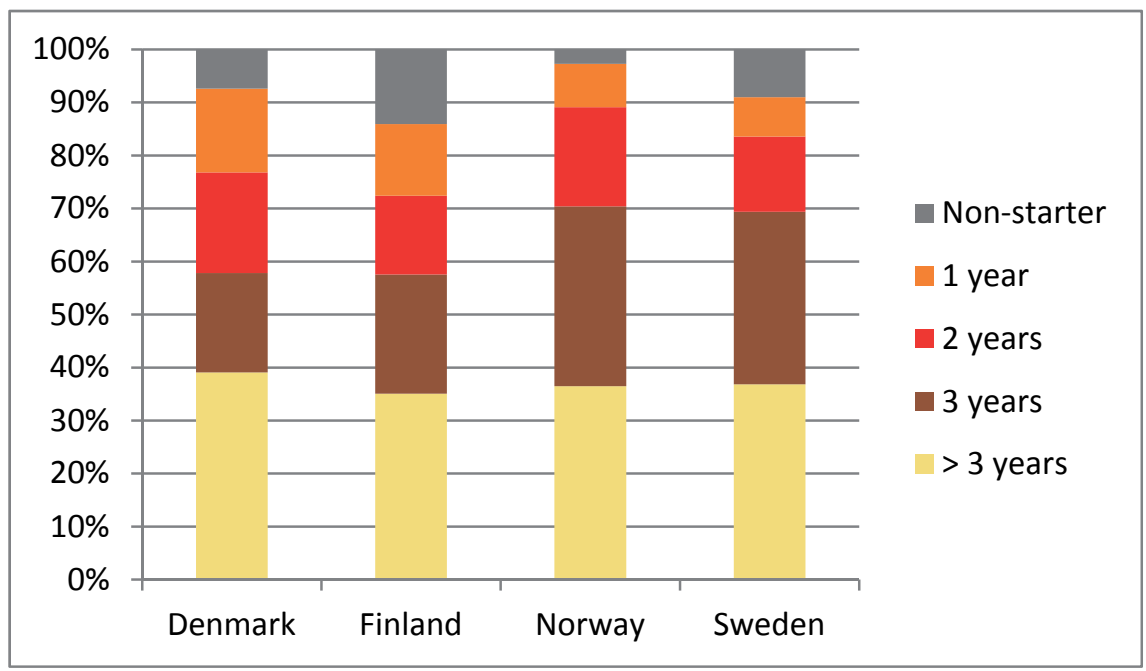

Note: The figure is based on the information provided in Tables 5.2 to 5.6. 
The information provided in Figure 5.6 is presented in a rescaled format in Table 5.7 in order to illustrate the corresponding situation when account is made for the fact that the non-completion rate among 21-yearolds differs substantially across the four countries (see Chapter 2). In both Denmark and Norway, more than one-fifth of the youth population continue in post-compulsory education for at least 3 years but fail, nonetheless, to achieve an upper secondary degree by age 21 . The corresponding share for Finland and Sweden is just above 10\%. The cross-country situation is much more similar when, instead, comparing the share of youngsters dropping out early from post-compulsory education: almost $14 \%$ of Danish youth follow early school-leaving tracks, compared to about $8 \%$ for Finland and Norway, and close to $6 \%$ for Sweden.

Table 5.7: Distribution of non-completers across stylized pathways by number of initial years spent in post-compulsory education before dropping out, for the four Nordic countries, \%-share of the full youth population

\begin{tabular}{|c|c|c|c|c|}
\hline \multirow{2}{*}{$\begin{array}{l}\text { Number of initial years in post- } \\
\text { compulsory education }\end{array}$} & \multicolumn{4}{|c|}{$\%$-share of the full youth population } \\
\hline & Denmark & Finland & Norway & Sweden \\
\hline$>3$ years & 14.6 & 6.3 & 10.8 & 5.9 \\
\hline 3 years & 7.0 & 4.1 & 10.1 & 5.2 \\
\hline 2 years & 7.1 & 2.7 & 5.6 & 2.3 \\
\hline 1 year & 5.9 & 2.4 & 2.4 & 1.2 \\
\hline Non-starter & 2.8 & 2.5 & 0.8 & 1.4 \\
\hline $\begin{array}{l}\text { Non-completers' share in the full } \\
\text { youth population }\end{array}$ & 37.3 & 18.0 & 29.7 & 16.0 \\
\hline
\end{tabular}

Notes: The percentage shares displayed in Figure 5.6 as recalculated in relation to the full youth population of each country.

Next, we turn from the activity dominating the start of each stylized pathway to the activity dominating the end years of each pathway. As shown in Figure 5.7, the stylized post-compulsory-school pathways dominated by continuous engagement in full-time education cover more than one-third of the non-completers in all four countries. These young people spend most of their years from age 16 up to age 20 as full-time students but fail, nonetheless, to finalise their upper secondary education by age 21 . The share of young non-completers spending most of their early post-compulsory-school years in education is largest in Denmark (39\%) and lowest in Finland (32\%).

Denmark also has the largest share of young non-completers following post-compulsory-school tracks moving them early into working life (close to 36\%), followed by Finland (30.5\%). In Norway and Sweden, young noncompleters are less likely to leave school for employment. Taken together, these early education- and employment-dominated pathways comprise $75 \%$ of the Danish non-completers. The corresponding share of non- 
completers spending most of their years from age 16 up to age 20 in either school or work is $66 \%$ for Finland, $64 \%$ for Norway and $62 \%$ for Sweden.

Figure 5.7: Distribution (\%-share) of young non-completers across stylized pathways by main activity after leaving post-compulsory education, for the four Nordic countries

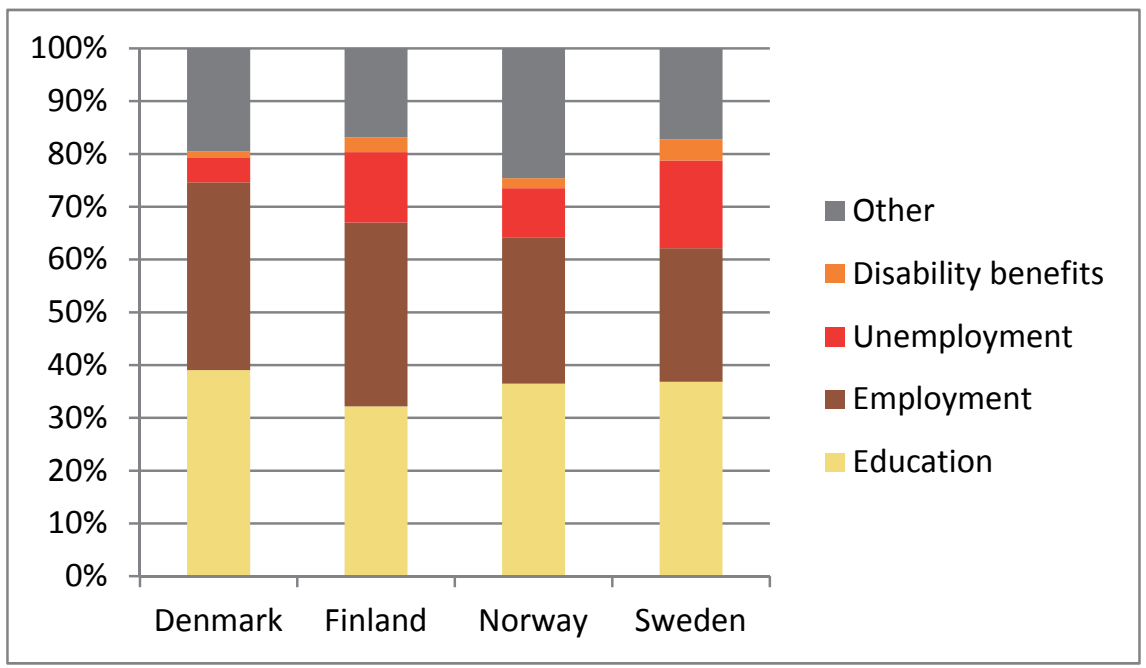

Note: The figure is based on the information provided in Tables 5.2 to 5.6.

Swedish non-completers are more likely to spend time in (registered) unemployment after having dropped out from upper secondary education than are non-completers in the other three Nordic countries: an average of close to $17 \%$ of Swedish non-completers end up in unemployment tracks before turning 21, compared to about $12 \%$ for Finland, some $9 \%$ for Norway and less than 5\% for Denmark. Most Swedish noncompleters in unemployment tracks have spent three years in upper secondary education before showing up as unemployed jobseekers. The relatively large share of Finnish non-completers in unemployment tracks is mainly due to the weak employment prospects of low-educated youth who tried to enter the labour market in the high-unemployment years of the 1990s. In the two younger Finnish cohorts, the shares of non-completers experiencing early spells in unemployment are similar to those of Danish and Norwegian non-completers.

Relatively few non-completers follow pathways shifting them already at an early age into disability arrangements. The highest share (an average of 4\%) is observed for Sweden. Much larger shares of young noncompleters show up in trajectories dominated by (unknown) activities outside both education and the labour force. This share is notably high 
(close to 25\%) for Norway. In the other three countries, it is clearly lower but still of a remarkable size: about 19\% for Denmark and Finland, and some $17 \%$ for Sweden.

Consider next the same distribution, now related to the full youth population of each country. After this rescaling, we find that about $13 \%$ of Danish youth shift early into employment tracks, which typically means that they lack an upper secondary degree still when aged 21. In Norway, the corresponding share is $8 \%$, in Finland $6 \%$ and in Sweden only $4 \%$. Denmark and Norway also come out with a larger share of the youth population (7.3\%) following early tracks ending with the young person withdrawing from both education and the labour market already before turning 21. In Finland and Sweden, such tracks are much less common. When related to the full youth population, the country-specific shares of youth ending up, already at an early age, in unemployment or disability become quite similar in size. Indeed, Finland, Norway and Sweden have about the same share of youth (2.4-2.8\%) moving early into registered unemployment - and non-completion of an upper secondary degree. The share for Danish youth is smaller, 1.7\%, but not as different as the comparison across non-completers suggests (in Figure 5.7).

\begin{tabular}{|c|c|c|c|c|}
\hline \multirow{2}{*}{$\begin{array}{l}\text { Main activity after leaving } \\
\text { post-compulsory education }\end{array}$} & \multicolumn{4}{|c|}{$\%$-share of the full youth population } \\
\hline & Denmark & Finland & Norway & Sweden \\
\hline Continue in education & 14.6 & 5.8 & 10.8 & 5.9 \\
\hline Employment & 13.3 & 6.3 & 8.2 & 4.0 \\
\hline Unemployment & 1.7 & 2.4 & 2.8 & 2.7 \\
\hline Disability benefits & 0.5 & 0.5 & 0.6 & 0.6 \\
\hline Other (inactivity) & 7.3 & 3.0 & 7.3 & 2.8 \\
\hline $\begin{array}{l}\text { Non-completers' share in the } \\
\text { full youth population }\end{array}$ & 37.3 & 18.0 & 29.7 & 16.0 \\
\hline
\end{tabular}

Notes: The percentage shares displayed in Figure 5.7 as recalculated in relation to the full youth population of each country.

Hence, the main implication of the large difference among the 21-yearolds in non-completion rates between especially Denmark, but also Norway, on the one hand, and Finland and Sweden, on the other hand, is that a notably larger share of Danish and Norwegian youth continue straight in post-compulsory education for typically three or more years, but without completing an upper secondary degree by age 21 , or leave school for work before having graduated. These differences are likely to be basically due to the institutional setting, in particular the organisation of vocational training. The shares of early dropouts going into typical NEET paths are more similar across the four countries. 


\section{Labour market outcomes as young adults}

Our analyses have so far focused on exploring young people's main activities and school-to-work-transition patterns over the five years following upon completion of compulsory school, that is, from age 16 up to age 20. A logical next step is to ask: What happens to these youngsters after they have turned 20? What kind of main activities - studying, employment, unemployment, disability arrangements or other types of inactivity - are they mostly engaged in as young adults? Can we observe distinct and rather stable differences in this respect across genders and/or between those differing in their educational background? Or is it possible that these later outcomes are, by and large, quite similar for young men and women, as well as for early and later completers of a post-compulsory educational degree and, possibly, even for noncompleters, i.e. those with no exam beyond primary education still as a young adult? Last, but not least, can we identify clear-cut similarities or dissimilarities in all these important dimensions across the four Nordic countries under study? This chapter sets out to provide answers to these key questions.

\subsection{Main activities beyond age 21 - all young people}

We start by recalling the overall pattern of labour market outcomes for young adults based on pooled information on our three country-specific youth cohorts (16-year-olds in 1993, 1998 and 2003). In other words, we first re-report in which main activities the young people covered by our national datasets are engaged at three different ages $-21,26$ and 31. But instead of repeating the country-specific graphs contained in Figures 2.1a to $2.1 \mathrm{~d}$ of Chapter 2, we now present the same information from a slightly different angle. Then we refine this descriptive information in an attempt to unravel to what extent the economic situation is possibly reflected in the labour market outcomes of our youth cohorts under scrutiny. 


\subsubsection{Reproducing the general picture from a different angle}

In particular, Figure 6.1 contains three graphs with the first graph providing information on the allocation of each country's young people across our five main activity categories - studying, employment, unemployment, disability arrangements and other types of inactivity - when they turned 21. The next (middle) graph gives the corresponding information five years later, at age 26 . The bottom graph, finally, displays the situation ten years later, at age 31. Each graph then highlights crossNordic similarities and dissimilarities in young people's activity shares at these particular ages. A top-down comparison of the three age-specific distributions sheds, in turn, light on the changes in activity shares within countries when moving from age 21 to age 31 . Indeed, this line-ofcomparison is identical to the information provided in the countryspecific Figures 2.1a to $2.1 \mathrm{~d}$ of Chapter 2 .

Figure 6.1: Distribution (\%-share) of young people across main activities at age 21, 26 and 31, respectively, based on pooled information on all three youth cohorts, by country

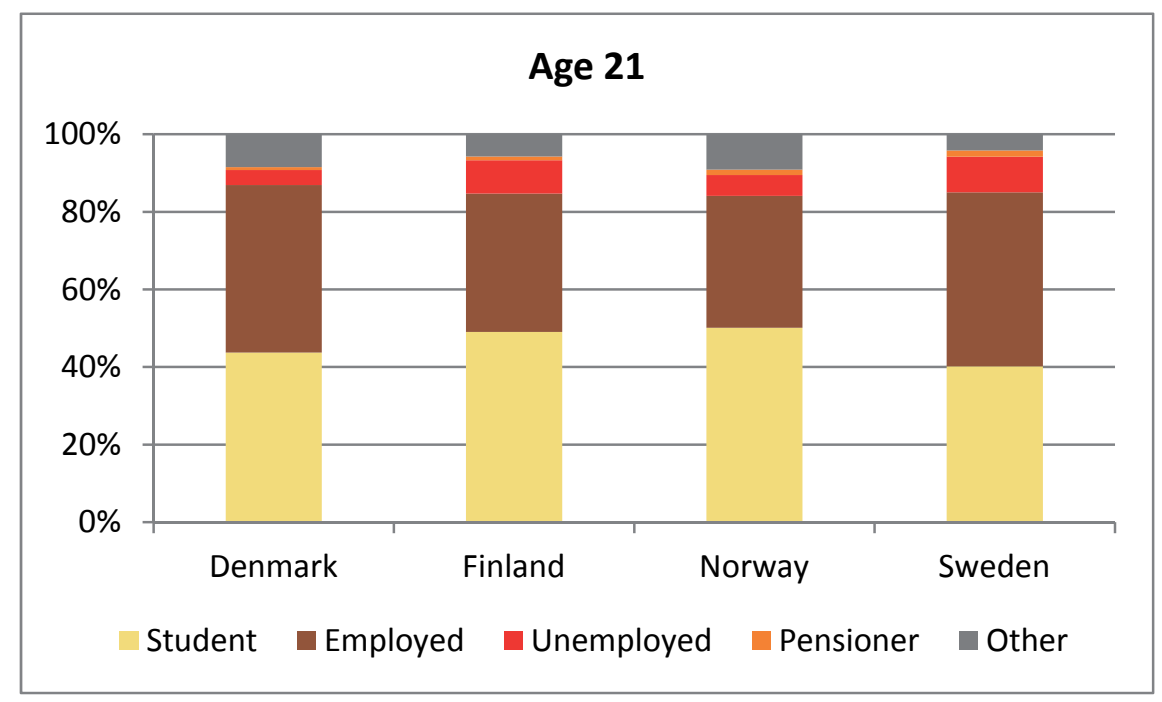



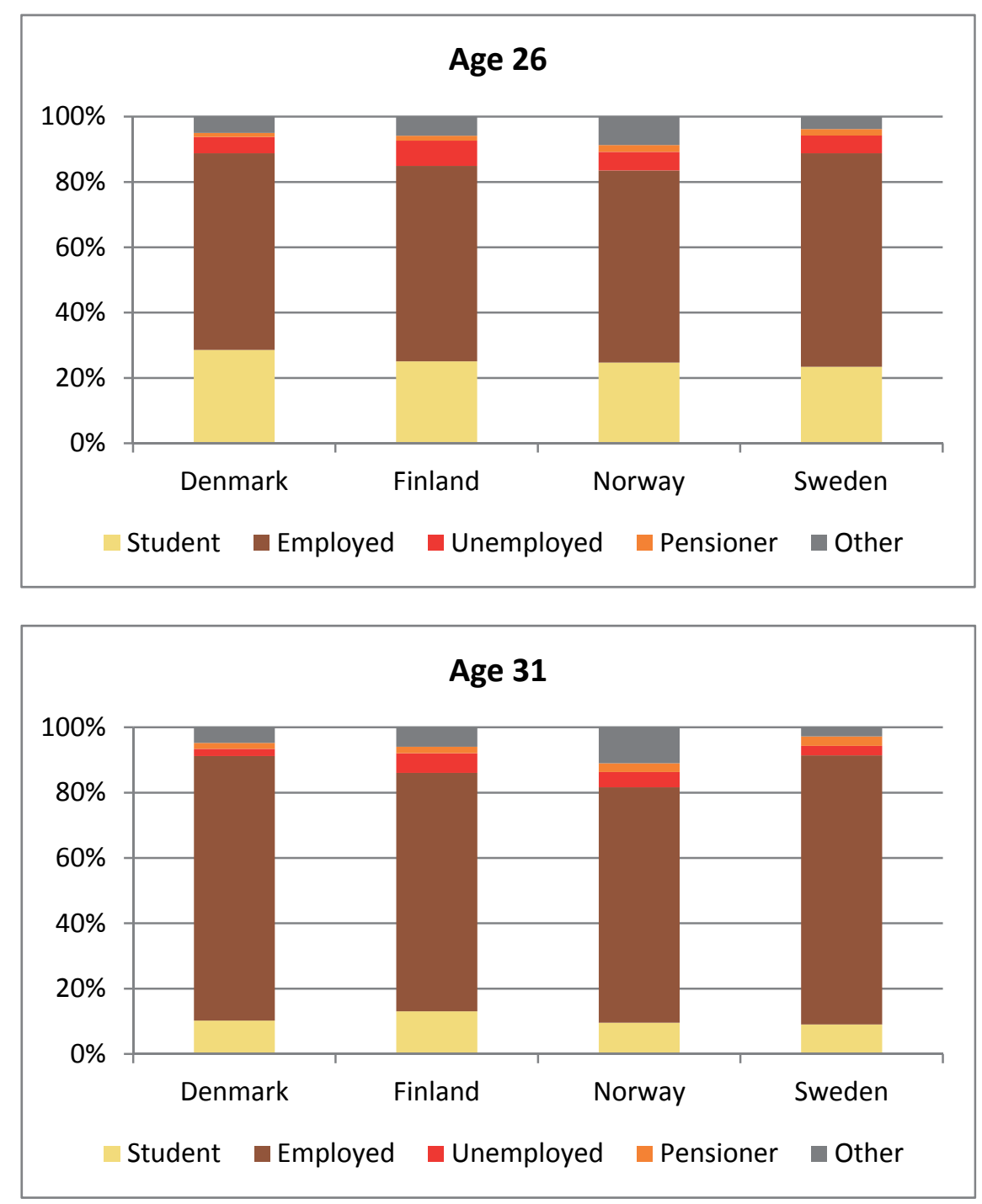

Note: For definitions of the five main activity groups, see Chapter 2.

Since Figure 6.1 contains the same basic information at ages 21, 26 and 31 as Figures 2.1a to 2.1d, albeit in a different mode, we here comment only briefly on the patterns displayed in the figure. First, the overall picture looks much the same across the four Nordic countries under study: rapidly declining shares in full-time education and growing shares in employment when young people grow older. This is, in effect, the most conspicuous change in activity shares occurring beyond age 21 .

Second, in all four countries there are non-negligible shares of young people experiencing unemployment, health problems moving them onto disability benefits, or other forms of inactivity excluding them from both 
education and working life. Although also these NEET shares change over time, the changes are rather small in magnitude with no systematic pattern discernible across the four countries.

Third, the situation at age 21 looks more or less the same in all four countries when adding up young people's shares in full-time education and employment, on the one hand, and in NEET activities, on the other hand. Moreover, this overall pattern is only marginally different five years later, at age 26 . While the distribution of young people across main activities appears to reveal more distinct cross-country differences by age 31 , when compared to the situation five or ten years earlier, we need to recall that the outcome at age 31 is based on information on one single cohort, viz. the oldest cohort representing young people who turned 16 in 1993.

\subsubsection{Cohort effects of the economic situation}

Next we refine the descriptive information provided in Figure 6.1. This exercise departs from the fact that we have information on later labour market outcomes for two of our three youth cohorts under scrutiny: the 1998 cohort of 16-year-olds can be followed up to age 26 (in 2008) while the 1993 cohort of 16-year-olds can be traced up to age 31 (in 2008). A main motivation for looking somewhat closer into these two cohorts is that they started their school-to-work transition in very different economic contexts. As referred to in the outset, we know from the literature that the prevailing economic situation tends to have farreaching career consequences for young labour market entrants. Accordingly, we might expect the labour market experiences in adulthood to be different for these two cohorts.

In Figure 6.1, the distribution across activities of our young people when aged 26 shows the average outcome for the 1993 and 1998 cohorts. In contrast, and as already underlined above, the distribution of 31-year-olds reflects the situation for the oldest (1993) cohort only. By splitting the information provided in the figure for age 26, we may undertake two additional comparisons potentially shedding light on the following questions: Does the distribution across main activities at age 26 look different for the "economic-bust" cohort of 1993 and the "economic-boom" cohort of 1998 ? Does the situation change markedly between age 26 and age 31 for the economic-bust cohort of 1993, or does it remain approximately unchanged? These two comparisons are undertaken in Figures 6.2 and 6.3. Since the economic recession in the early 
1990s hit all four Nordic countries under study, we would expect crosscohort differences to show up for all four countries.

According to Figure 6.2, the share of young people engaged in either full-time education or employment is, by age 26 , slightly higher in the 1998 "economic-boom" cohort than in the 1993 cohort. However, this holds true for Denmark and Finland only. In Denmark, this higher "activity" share of the 1998 cohort is due to full-time studying still at age 26 being more common in the 1998 cohort, whereas the share in working life is of the same magnitude in the two cohorts. In Finland, on the other hand, the 1998 cohort is by age 26 more engaged in both full-time studies and employment than was the 1993 cohort when aged 26.

The situation looks different for Norway and Sweden. For both countries, we observe a small decline across the two cohorts in the share of full-time students and a slight increase in the share in employment. For Sweden, these two opposite-signed changes are of much the same magnitude, for which reason we see principally no difference across the two cohorts in the total share of 26-year-olds in either education or employment. For Norway, in contrast, the drop in the share of full-time students more than outweighs the concomitant increase in the share of employed. This results in an "activity" share among 26-year-old Norwegians that is lower in the 1998 cohort than in the 1993 cohort.

Figure 6.2 further indicates that the share of 26-year-olds in (registered) unemployment was clearly lower in the 1998 cohort. This holds true for all four countries and is, most likely, due to a favourable economic situation in combination with increased volumes of active labour market policies. Simultaneously, however, the share of 26-year-olds on disability benefits or in other types of inactivity appears to be of much the same size in the two cohorts (Finland) or even larger in the 1998 economic-boom cohort (notably in Norway, but also in Denmark and Sweden). All in all, Figure 6.2 seems to suggest that the prevailing business cycle has, at most, been reflected in young people's activities when it comes to studying and working, including unemployment, whereas withdrawal from both education and the labour market is mainly the result of other processes and mechanisms. 
Figure 6.2: Distribution (\%-share) of young people across main activities at age 26, by country: comparison of the "economic-bust" cohort of 1993 with the "economic-boom" cohort of 1998
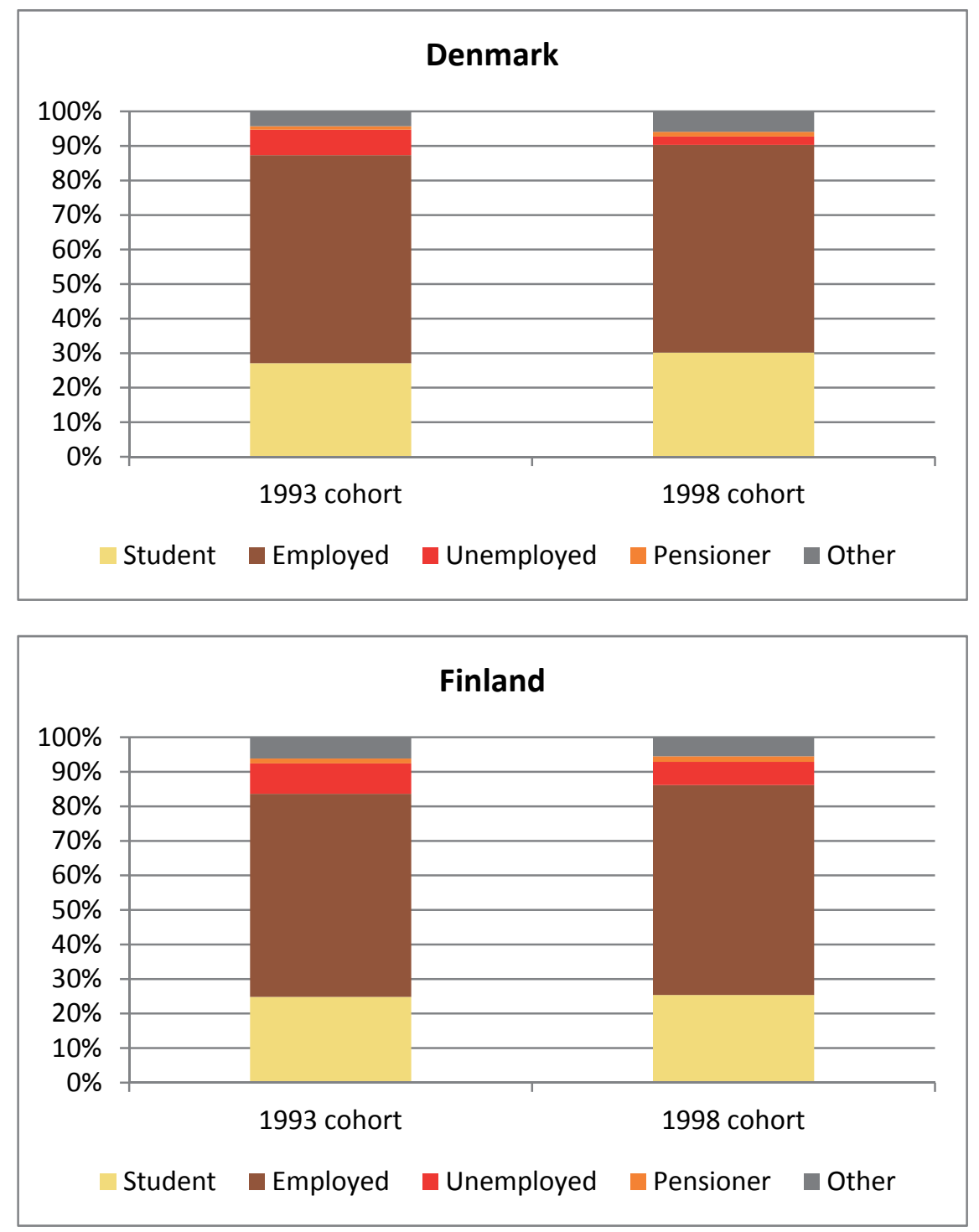

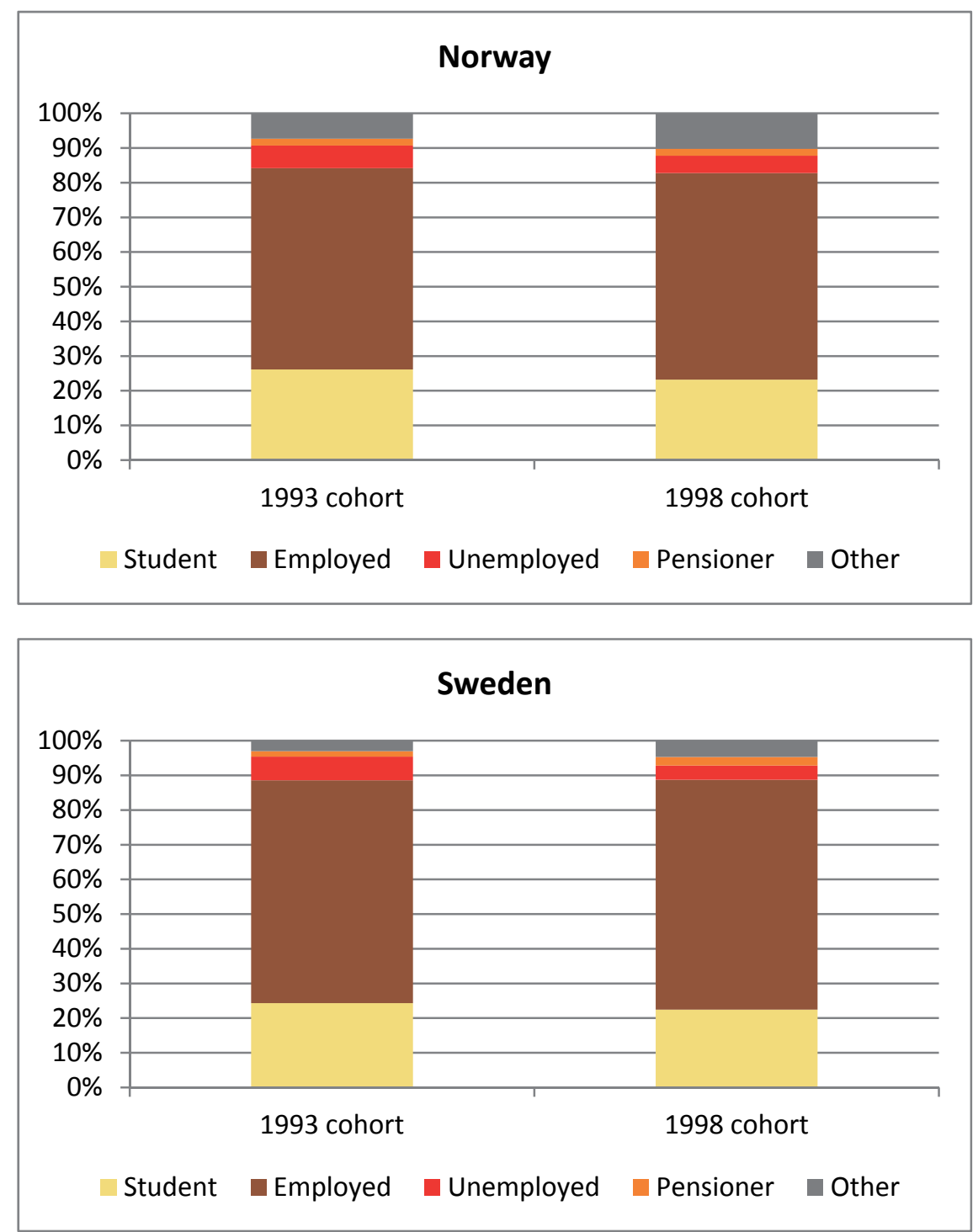

Note: For definitions of the five main activity groups, see Chapter 2.

Figure 6.3, finally, shows that the overall labour market outcome of the 1993 "economic-bust" cohort improved over the 5-year period from age 26 up to age 31. In Sweden, more than eight out of ten (82\%) of the cohort's young people were working at age 31 with an additional $9 \%$ still being enrolled in education. Hence, $91 \%$ of the Swedes belonging to this cohort were either studying or working when aged 31 (compared to close to $89 \%$ when aged 26). In other words, the situation of the Swedish 1993 cohort had, by age 31, turned very similar to the situation of the Danish 1993 cohort in terms of both employment and education. Also 
the Finnish 1993 cohort had by age 31 experienced a notable improvement in its employment situation which, nonetheless, remained notably weaker (73\%) than for the Danish and Swedish 1993 cohorts. However, this lower employment level is not necessarily entirely due the economic recession and the high unemployment levels of the 1990s: as has become evident also in the previous chapters, Finland is throughout characterised by a lower share of employed, when compared to the other three Nordic countries under study.

Figure 6.3: Distribution (\%-share) of young people across main activities at age 26 and 31, respectively, by country: the "economic-bust" cohort of 1993
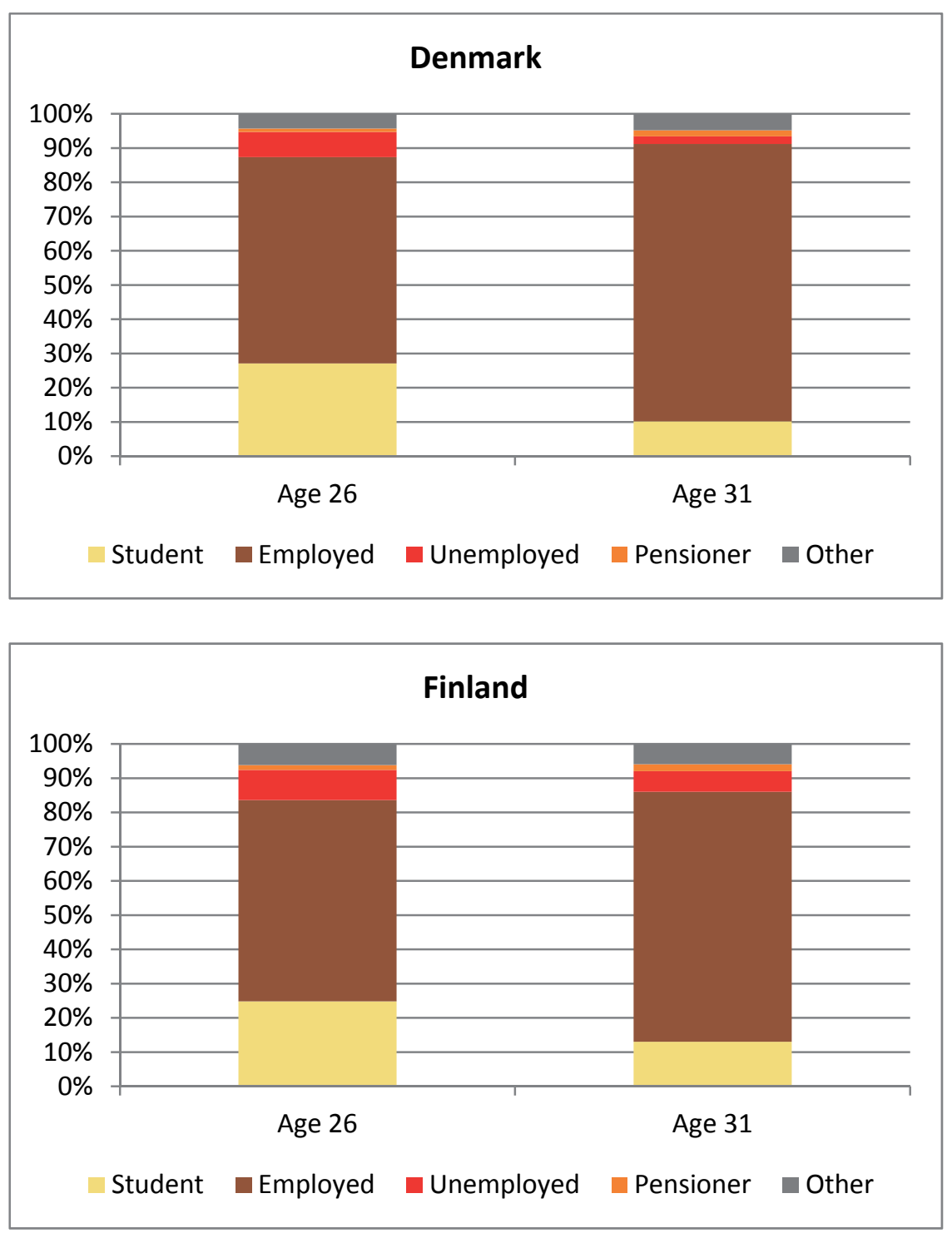

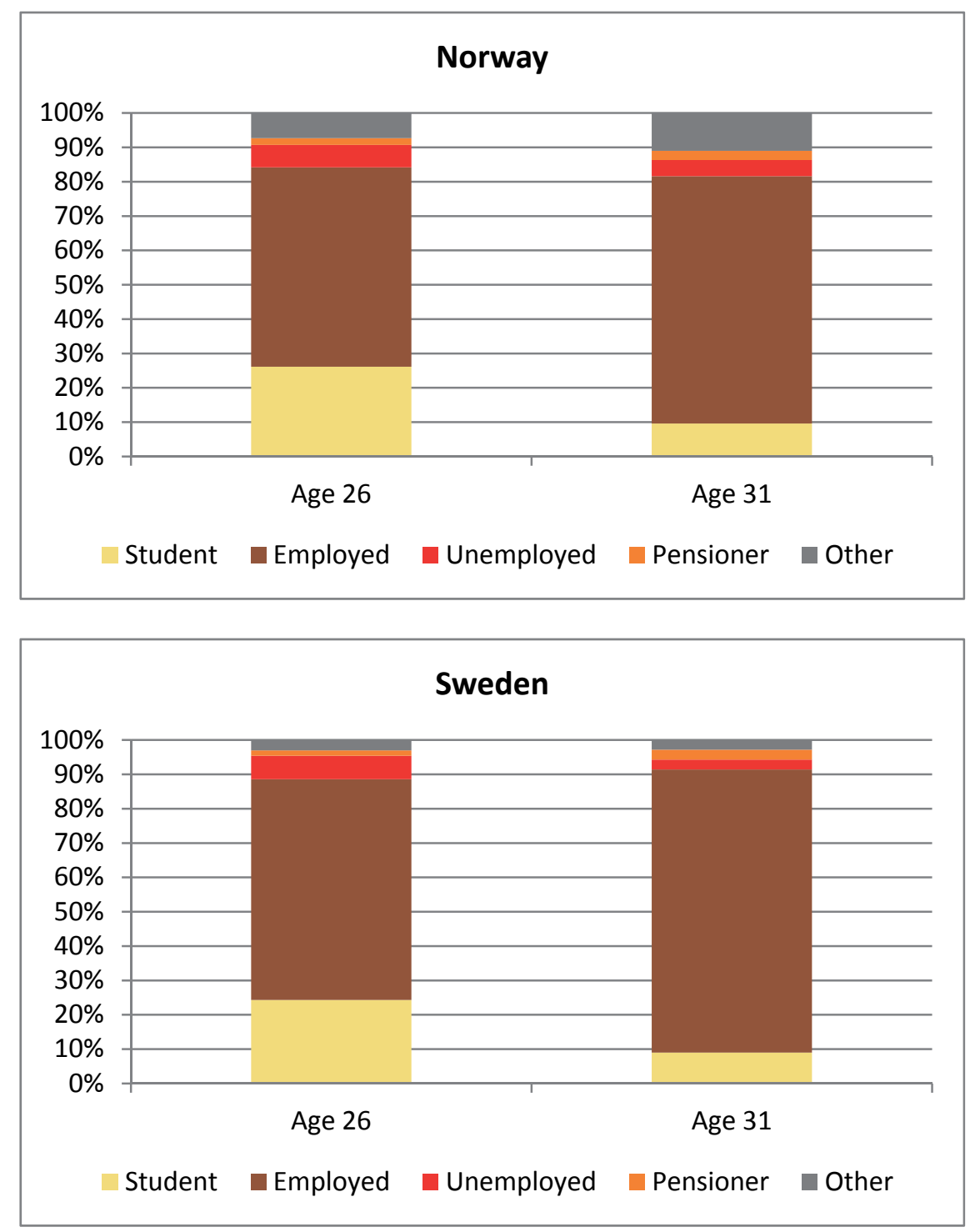

Note: For definitions of the five main activity groups, see Chapter 2.

In parallel with improving employment up to age 31, the 1993 cohort of Denmark, Finland and Sweden experienced a decline in the share of unemployed jobseekers. But simultaneously all three countries also saw an increasing share of the cohort's young people moving outside both education and the labour market, when comparing the situation at age 26 to that prevailing five years later, at age 31 . This increase is, for the most part, explained by a higher share receiving disability benefits.

A slightly different pattern emerges for the Norwegian 1993 cohort. While the share of the cohort's young people moving into working life 
increased between age 26 and 31 also in Norway, this growth could not compensate for the concomitant drop in the share still enrolled in fulltime education. As a consequence, the cohort's "activity" (education + employment) share was marginally lower at age 31 than at age 26 . Simultaneously, the share of the cohort's young people moving outside both education and the labour force increased quite markedly, from about $9 \%$ at age 26 to almost $14 \%$ at age 31 . Moreover, only a minor part of this increase is explained by a growing number of the cohort's young adults moving into disability arrangements. Instead, the main explanation seems to be that they withdraw into other types of inactivity not covered by the large administrative registers from which our national datasets are compiled.

\subsubsection{Main findings}

In this sub-chapter, we have addressed two interconnected questions: Does the distribution across main activities at age 26 look different for the "economic-bust" cohort of 1993 and the "economic-boom" cohort of 1998 ? Does the labour market situation change between age 26 and age 31 for the economic-bust cohort of 1993, or does it remain approximately unchanged?

Our descriptive analysis based on information for the full youth cohorts cannot provide clear-cut answers to these questions in the sense that we see no systematic cross-country trends in activity shares at age 26 for the economic-bust and economic-boom cohorts. In particular, the share in employment by age 26 is slightly higher in the 1998 cohort than in the 1993 cohort for Finland, Norway and Sweden, but not for Denmark. The share enrolled in full-time education still at age 26 is higher in the Danish and Finnish 1998 cohorts, whereas the opposite holds true for Norway and Sweden. Adding up the shares in education and employment reveals no conspicuous differences between the two cohorts, either. Instead, we see a slight increase in this "activity" share across the Danish and Finnish cohorts, no change across the Swedish cohorts and a decline across the Norwegian cohorts. While the share in (registered) unemployment at age 26 is, indeed, lower in the 1998 cohort in all four countries, this change seems to have occurred at the expense of a much higher (Norway) or slightly higher (Denmark and Sweden) share of 26year-olds in the 1998 cohort standing outside both education and the labour force. For Norway, the results further indicate that this conspicuous increase in the share of young people withdrawing from both educa- 
tion and the labour force has been fed not only by a flow from unemployment but also from full-time education.

The country-specific patterns are more similar when comparing the labour market situation of the 1993 cohort of 16-year-olds at two points later in life, viz. at age 26 and age 31. In all four countries, we observe an improvement in the cohort's labour market situation between age 26 and 31 in terms of more employment and less unemployment. Finally, while the share outside both education and the labour force reveals an upward trend in all four countries, this change up to age 31 has generally been quite modest, except for the Norwegian 1993 cohort.

However, these weak signs of the deep economic recession of the early 1990s having had an impact on the labour market outcomes of the 1993 cohort of 16-year-olds do not mean that their situation was unaffected by the difficult employment situation that prevailed for several years also after the start of the economic recovery. First and foremost, we need to recall that our data measures registered unemployment. As shown and discussed at length in Chapter 1, this measure is likely to underestimate the prevalence of unemployment among young people: many of them do not fulfil the conditions for signing on, or then they may choose not to register if not being eligible for receiving unemployment benefits. However, it might also be that the economic crisis affected specific groups of young persons, instead of influencing all young people about to enter the labour market. In Finland, for instance, the recession started in male-dominated export industries, which resulted in surging male unemployment rates and widespread destruction of especially low-skilled jobs often occupied by low-educated youngsters. Next, we therefore deepen our analysis in two respects: first, by comparing the situation of young men and women, and second, by contrasting the outcomes of completers and non-completers of an upper secondary degree by age 21 .

\subsection{Main activities beyond age 21 - young men vs. young women}

Next, we compare the labour market outcomes in adulthood across genders: Are the labour market experiences of young men and young women distinctly different at age 21, 26 and/or 31? Can we identify a stronger impact on either gender of the economic crisis in the early 1990s? 


\subsubsection{Labour market outcomes by gender at three specific age points}

A logical way to start this male-female comparison is to split the information provided in Figure 6.1 by gender. This is done in Figure 6.4 with the structure of the figure now emphasising, first and foremost, withincountry differences rather than between-country differences.

Figure 6.4: Distribution (\%-share) of young people across main activities at age 21, 26 and 31, respectively, based on pooled information on all three youth cohorts, by gender and country
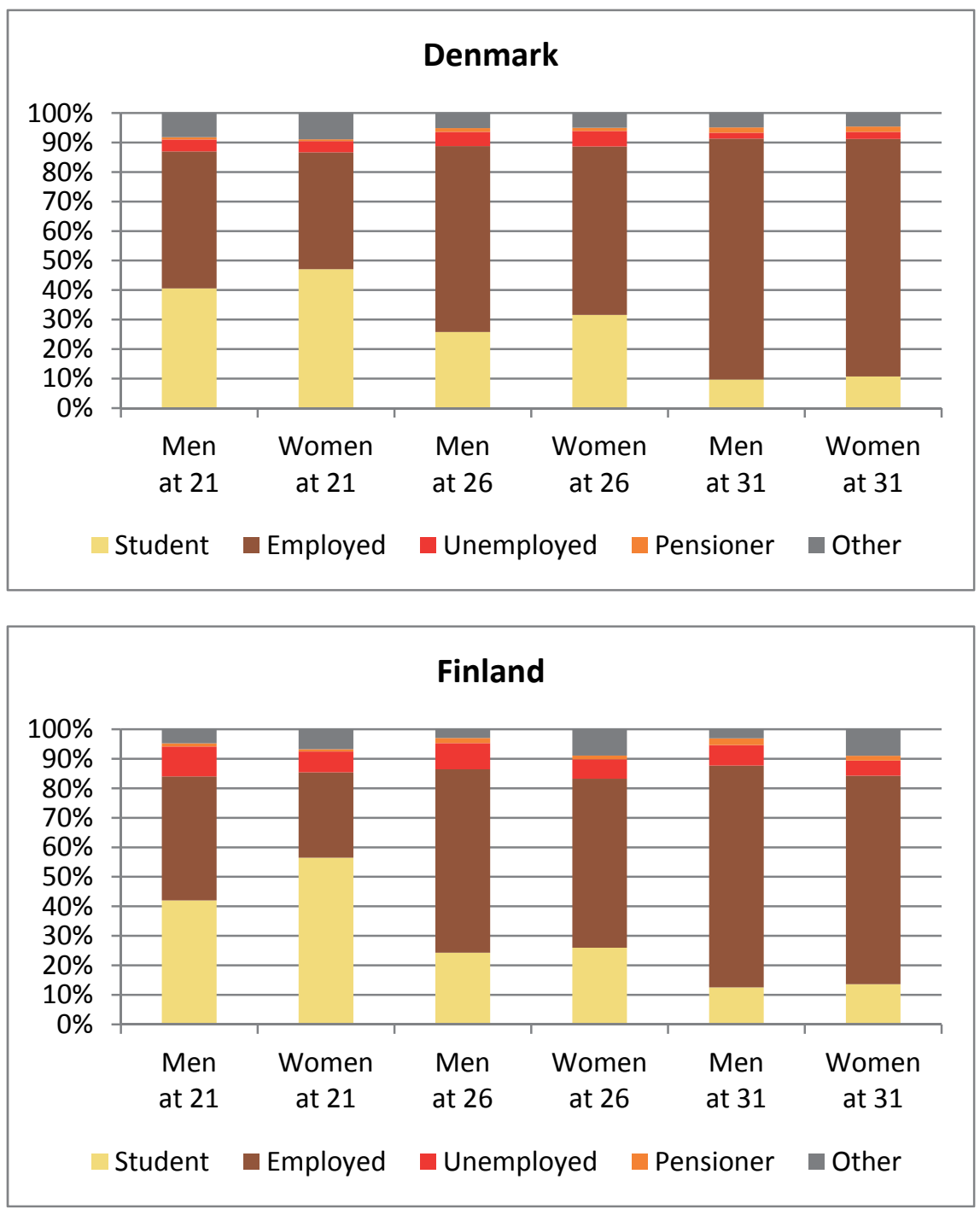

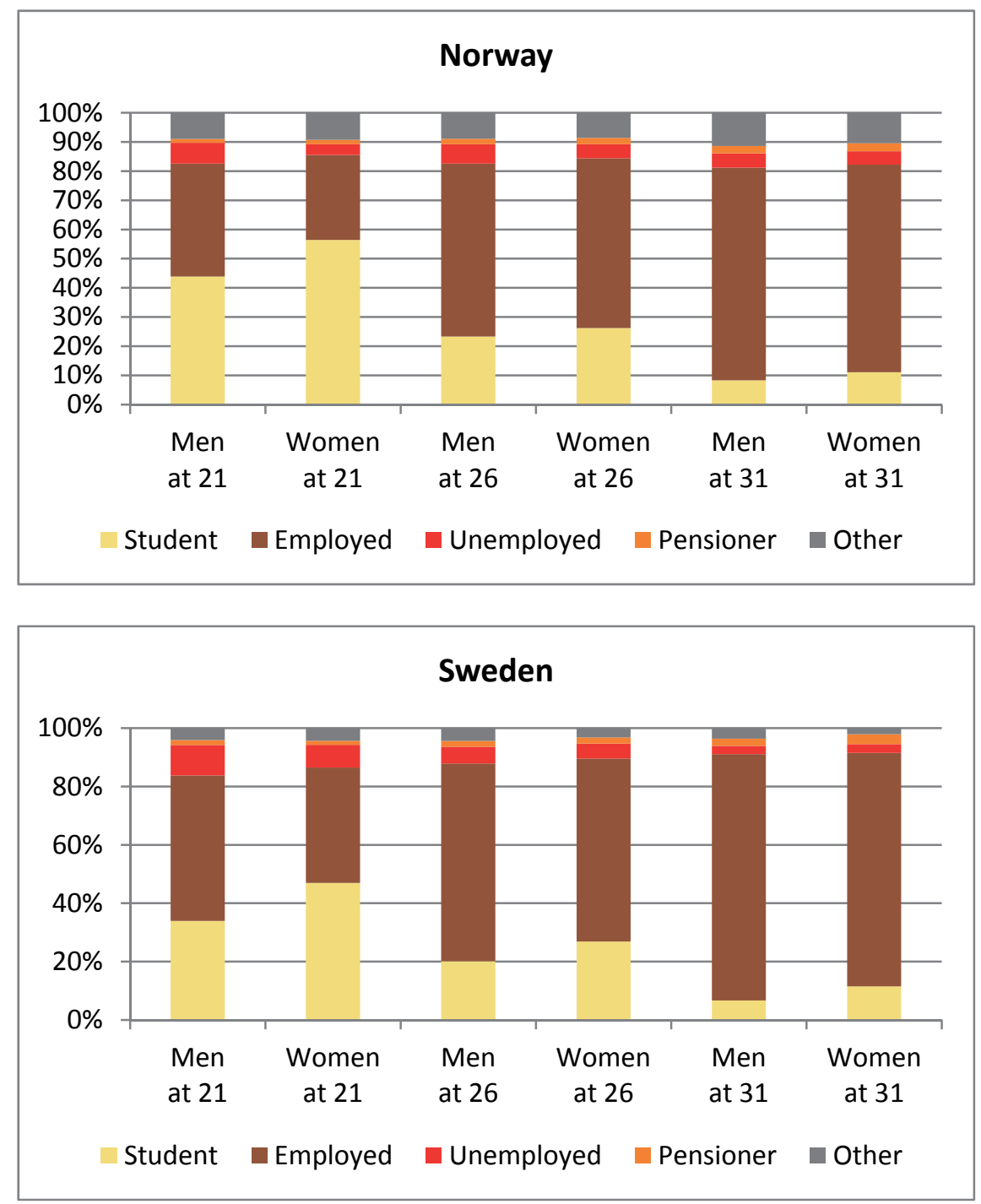

Note: For definitions of the five main activity groups, see Chapter 2.

The first graph of Figure 6.4 highlights the situation for Denmark. We see basically no distinct differences across genders among young Danes, irrespective of whether we compare their situation at age 21 , age 26 or age 31 . The only divergence in their distributions across main activities concerns the higher share of young women continuing in full-time education and a correspondingly higher share among young men moving into working life, a difference pointed out already in previous chapters. However, also this cross-gender difference seems to have almost disappeared by age 31 . 
In the other three countries, there are clearly more differences in the distribution across activities between young men and young women. While much larger shares of young women are in full-time education at age 21 , this difference across genders diminishes steadily over time, but it does not come close to vanishing as in Denmark. Likewise, also the gender gap in employment shares observed at age 21 shrinks over time, but still by age 31 working is more common among men than among women. Nonetheless, taken together, larger shares of young women than of young men are in either education or work. This holds true at all three age points for Norway and Sweden, but only at age 21 for Finland. At age 26, as well as at age 31, the share of Finnish women either working or studying is lower than for Finnish men.

These gender differences in education and employment shares are, of course, mirrored by corresponding differences across genders in relation to NEET activities. However, in this respect it is much harder to find common patterns for Finland, Norway and Sweden. In particular, while all three countries are characterised by a lower share in unemployment among young women than among young men aged 21, this gap prevails up to age 31 in Finland, disappears by age 31 in Norway, and is reversed by age 31 in Sweden. A similar pattern is, in effect, discernible across genders, ages and countries for those on disability benefits. For other types of inactivity, we observe for Finland a larger share for young women than for young men with, moreover, the inactivity share among young Finnish women increasing rapidly with age. The inactivity share is large and growing with age also in Norway but, in contrast to Finland, this seems to hold true for both genders. For Sweden, on the other hand, the inactivity share shrinks with age for both genders, more for young women than for young men. By age 31, a clearly larger share of Swedish men than of Swedish women belongs to the dumping category of "other" inactivity.

\subsubsection{Gender-specific cohort effects of the economic situation}

As a second step in our cross-gender comparisons, we repeat the split by cohort of the distributive information provided for age 26 in an attempt to unravel whether or not the economic situation at the time of labour market entry has eventually affected young men and young women differently when it comes to their distributions over main activity categories. The results of this exercise are displayed in Figure 6.5. 
Figure 6.5: Distribution (\%-share) of young people across main activities at age 26, by gender and country; comparison of the "economic-bust" cohort of 1993 with the "economic-boom" cohort of 1998
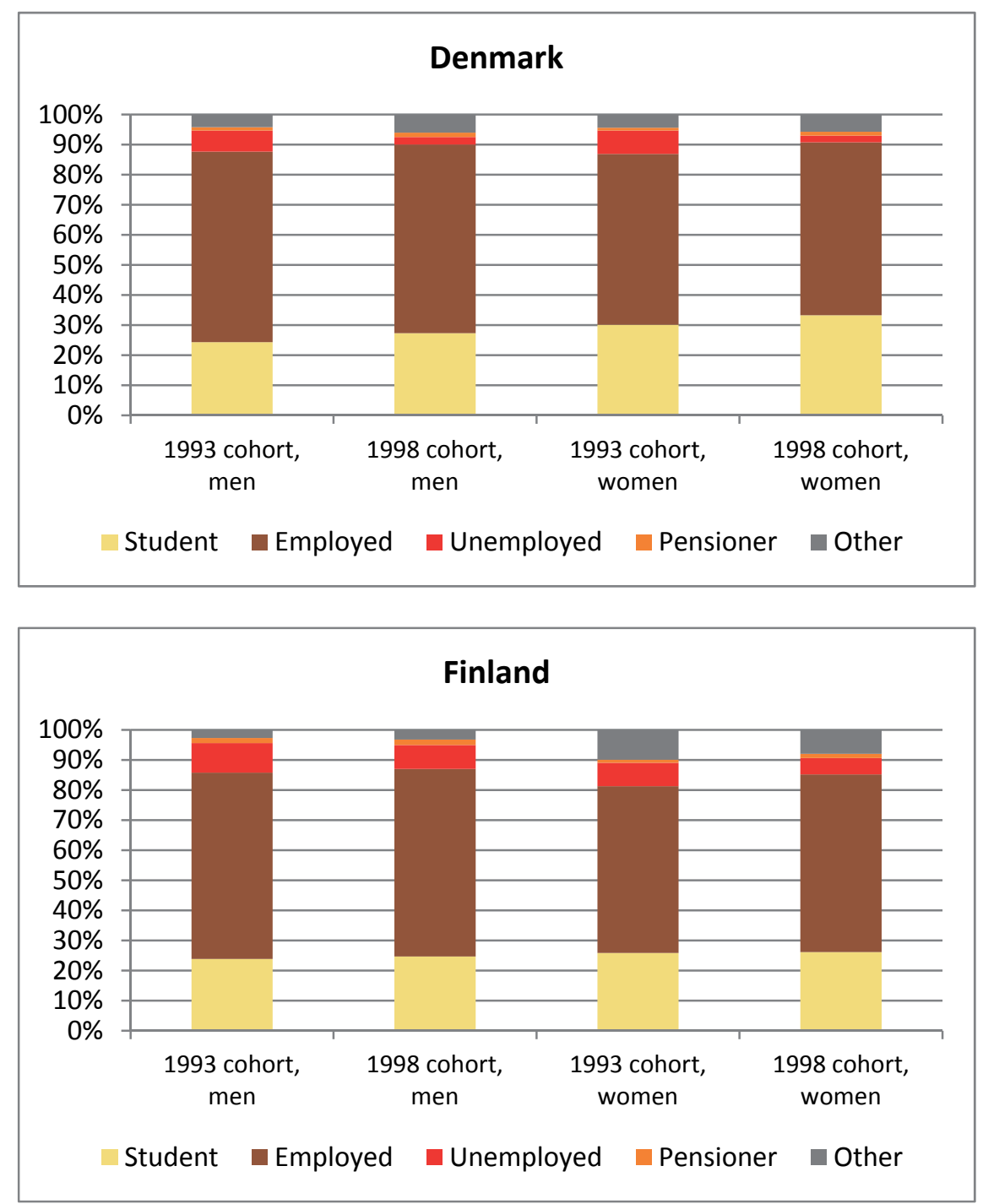

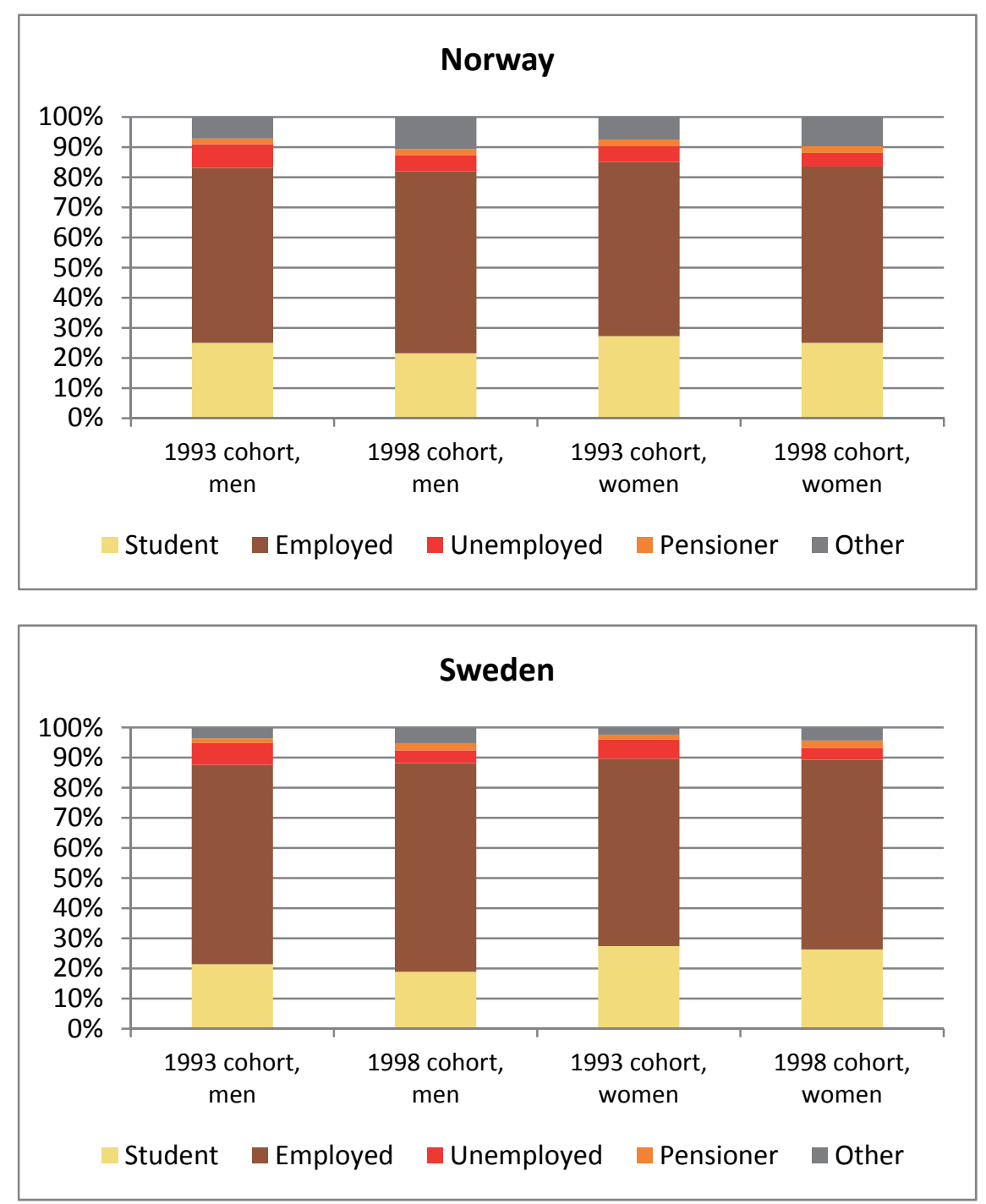

Note: For definitions of the five main activity groups, see Chapter 2.

In the previous sub-chapter focusing on all young people, we noted that the share of young people in either education or employment is somewhat higher at age 26 in the 1998 "economic-boom" cohort, but only in Denmark and Finland. For Denmark, this higher "activity" (education + employment) share was found to be due to a larger share of full-time students in the 1998 cohort, whereas the share in working life appeared to be of the same magnitude in the two cohorts. As is evident in Figure 6.5 , this overall pattern shows up for both genders. For Finland, we found a slightly higher share in both education and employment among 26-year-olds belonging to the 1998 cohort. Also these patterns are re- 
peated for both genders. For Sweden, we identified opposite-signed but balanced changes in educational and employment shares across the two cohorts, resulting in approximately similar "activity" shares for the 1993 and 1998 cohorts. This pattern is discernible for both genders. For Norway, finally, we saw a similar but more unbalanced trend across the two cohorts. In particular, the increase in the share of employed was not enough to compensate for the concomitant decline in the share of 26year-olds enrolled in full-time studies, which showed up as a lower "activity" share in the 1998 cohort than in the 1993 cohort. Again, the same pattern holds true for both genders.

Common to all four countries was a share of 26-year-olds in (registered) unemployment that was lower in the 1998 cohort. Also in this respect we observe the same pattern for young men and young women. The cohort-specific shares of 26-year-olds on disability benefits or in other types of inactivity were found to reveal much more cross-country variation: for Finland, the inactivity share in the two cohorts was noted to be of much the same size, but for Denmark, Sweden and notably for Norway, the share of young people outside both education and the labour market was found to be larger in the 1998 than in the 1993 cohort. Again, the same cross-cohort pattern is discernible for both genders. Hence, the differences between the "economic-boom" and the "economic-bust" cohort in the distribution of young people across main activities pointed out in the previous sub-chapter do not show up differently among young men and young women; the same pattern emerges irrespective of gender. Moreover, this holds true for all four countries.

Finally, we take a gendered perspective on the distribution across main activities of the 1993 "economic-bust" cohort at two age points: when aged 26 and 31, respectively. Here, the main question is whether or not the labour market outcomes of males belonging to this particular cohort possibly evolved differently over these years, when compared to the experiences of their female counterparts. For this purpose, we split the information given in Figure 6.3 above to indicate the situation of young men vs. young women belonging to the 1993 cohort of 16-yearolds. This comparison is undertaken in Figure 6.6.

For Denmark, Finland and Sweden we found, based on Figure 6.3, that the overall labour market outcome of this "economic-bust" cohort improved over the 5-year period from age 26 up to age 31 . In all three countries, about nine out of ten of the cohort's young people were either studying or working at age 31 . Moreover, in all three countries, the share of young people registered as unemployed jobseekers declined up to age 31 , whereas the share outside both education and the labour force was 
marginally higher. Again, much the same overall pattern is discernible for both men and women: a decline in the share enrolled in full-time studies accompanied by a strong increase in the share in employment, less unemployment but slightly more inactivity. For both genders, this increase in the inactivity share by age 31 seems to be mainly due to a growing inflow into disability arrangements.

Figure 6.6: Distribution (\%-share) of young people across main activities at age 26 and 31, respectively, by gender and country, the economic-bust cohort of 1993
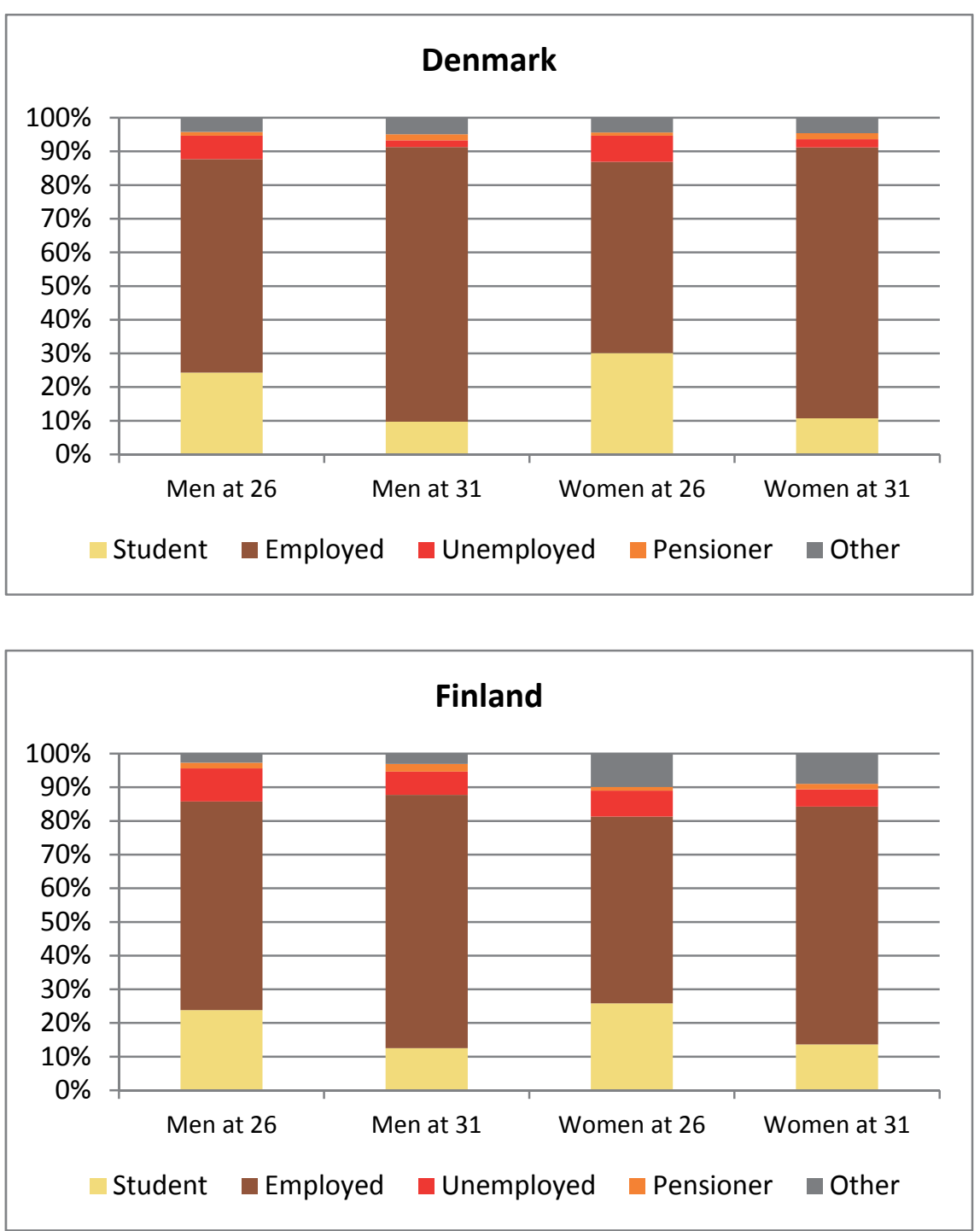

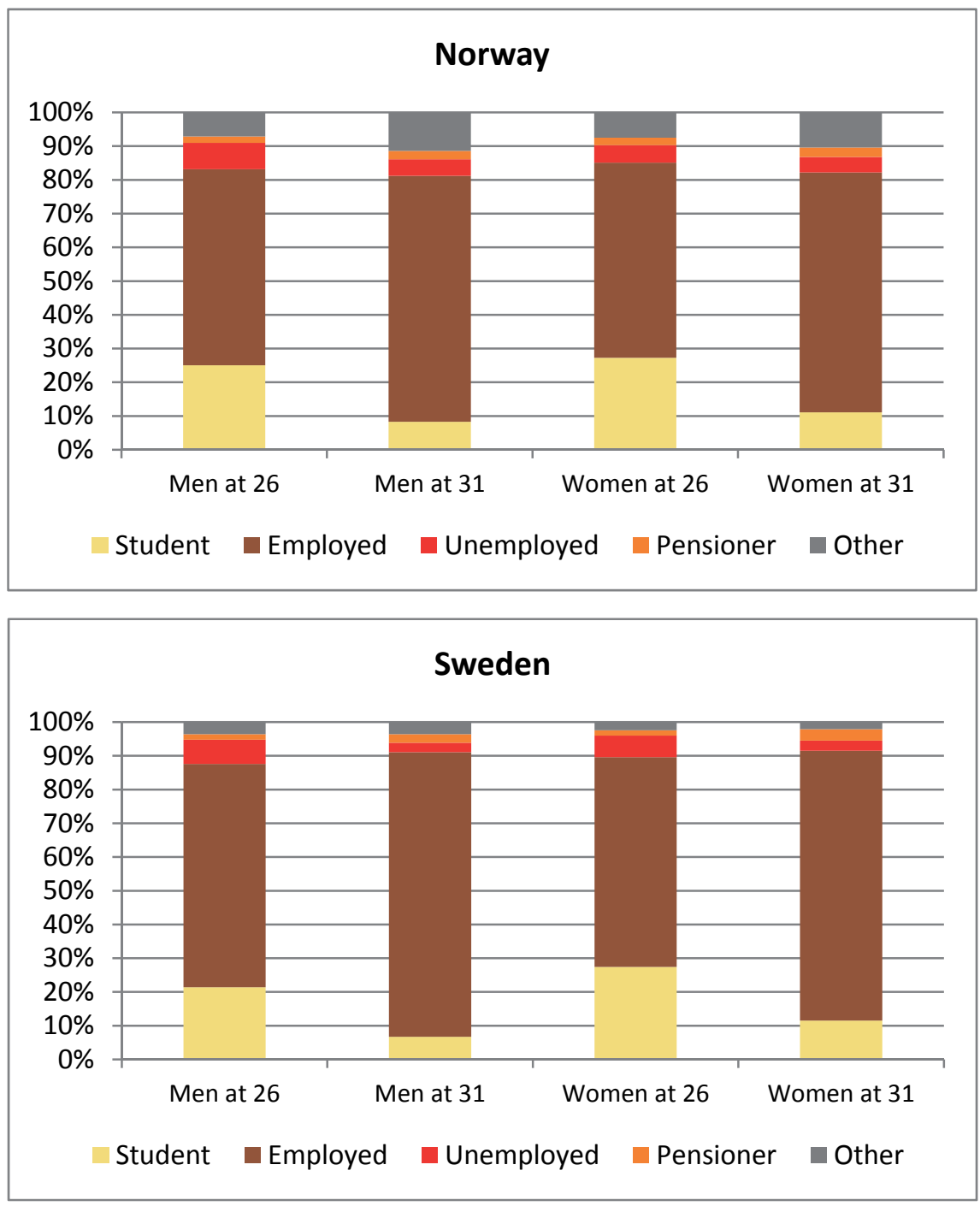

Note: For definitions of the five main activity groups, see Chapter 2.

Figure 6.3 mediated a slightly different pattern for the Norwegian 1993 cohort of 16-year-olds: despite a growth in the share of employed between age 26 and age 31 also in Norway, this increase could not compensate for the concomitant drop in the share of full-time students, which resulted in an "activity" share of the 1993 cohort that was lower at age 31 than at age 26. Moreover, while the share in unemployment declined, the share of the cohort's young people withdrawing from both education and the labour market had increased markedly by age 31 . As is evident from Figure 6.6, this overall pattern is strikingly similar for men and women. 


\subsubsection{Main findings}

In Chapter 2, we made a first comparison between genders also with respect to their distribution across main activities at age 21 . In this subchapter, we have expanded this comparison to two age points later in life: five years later, at age 26 , and ten years later, at age 31 . Doing so, we observe basically no differences across genders among young Danes, irrespective of whether we compare their labour market situation at age 21 , age 26 or age 31 . Indeed, also the higher share of young women continuing in post-compulsory education and the correspondingly higher share among young men moving already at a relatively early age into working life are differences that seem to have disappeared by age 31 .

We find much more differences in the gender distributions across main activities for the other three countries. The gender gap in the shares of full-time students and the employed narrows steadily over time but is discernible also at age 31 with enrolment in full-time education still being more common among young women and working being more common among young men. However, simultaneously we observe larger shares of young men than of young women outside education and work. This holds true at all three age points $(21,26,31)$ for Norway and Sweden, but only at age 21 for Finland. At both age 26 and age 31, the share of Finnish women neither working nor studying on a full-time basis is much higher than for Finnish men.

Attempts were also made to identify possible differences in the distribution of young men and young women across main activities due to changes in the economic environment. The simple exercises undertaken in this respect do not provide support for changes in the business cycle showing up differently among young men and young women. In other words, to the extent that changing business cycles are reflected in the overall distribution of young people across main activities, men and women seem to be affected in much the same way. This finding emerges for all four countries under study.

\subsection{Main activities beyond age 21 - completers vs. non-completers}

As a final step in this descriptive analysis of the labour market outcomes of our three youth cohorts when young adults, we turn the focus to a comparison of upper-secondary-school completers and non-completers. Again, completers refer to those young people in our three youth cohorts who succeeded in finalising their upper secondary education within five 
years after leaving compulsory school. The group of non-completers, in turn, comprises the remaining young people, that is, those with still no post-compulsory degree when reaching 21 years-of-age. This definition is valid throughout this sub-chapter. In the next sub-chapter (6.4), however, we will use a less strict definition in the sense that we allow for the fact that at least some of these non-completers do complete their upper secondary education, but only later on, after the age of 21 (cf. Chapter 2).

We start by comparing the distribution of completers and noncompleters across our five main activity categories when aged 21 . This comparison of labour market outcomes is then repeated five years later, at age 26 and, finally, ten years later, at age 31 . We conclude by returning to the question of the role played by the economic situation for later labour market outcomes, with the focus now being on a comparison of completers and non-completers.

\subsubsection{Situation five years later, at age 21}

Figure 6.7 illustrates to what extent completers (upper graph) and noncompleters (lower graph) are engaged in different main activities when aged 21, that is, five years after leaving compulsory school. From the figure it is evident that the distribution across main activities varies a lot when comparing those having completed to those not having completed an upper secondary degree by age 21 . Moreover, this holds true for all four Nordic countries under study. In several respects, there are also distinct cross-country differences among both completers and noncompleters, as pointed out already in Chapter 2 .

Broadly speaking, about $90 \%$ of the completers are either continuing in education or working when aged 21, with this share being slightly higher in Denmark and marginally lower in Finland. The corresponding situation for 21-year-old non-completers looks very different with a share in either full-time education or employment well below $70 \%$ for Finland, Norway and Sweden. Only in Denmark is this share clearly higher $(78 \%)$. From a within-country perspective, these differences in combined education and employment shares imply that the "activity" gap between 21-year-old completers and non-completers is comparatively large for Sweden (26\%-points), closely followed by Norway and Finland, with Denmark coming out with the smallest but still quite notable gap (15\%-points) (Table 6.1). 
The share of full-time students is by far highest (60\%) among Norwegian completers while the share of completers in employment is highest - and of a similar size (about 46\%) - in Denmark and Sweden. Conversely, the share of Danish and Swedish completers continuing in education when aged 21 is comparatively low, as is the share of 21-yearold Norwegian completers having entered working life. A distinctly different cross-country pattern emerges for the non-completers. In particular, the highest share of 21-year-old non-completers engaged in full-time studies is observed for Denmark (40\% compared to less than $30 \%$ in the other three countries). The situation is more or less the opposite when it comes to the non-completers' employment share: now Norway has the largest (close to 43\%) and Denmark the lowest (below 38\%) share.

Figure 6.7: Main activities at age 21 for completers and non-completers of an upper secondary education, based on pooled information on all three youth cohorts under study, by country

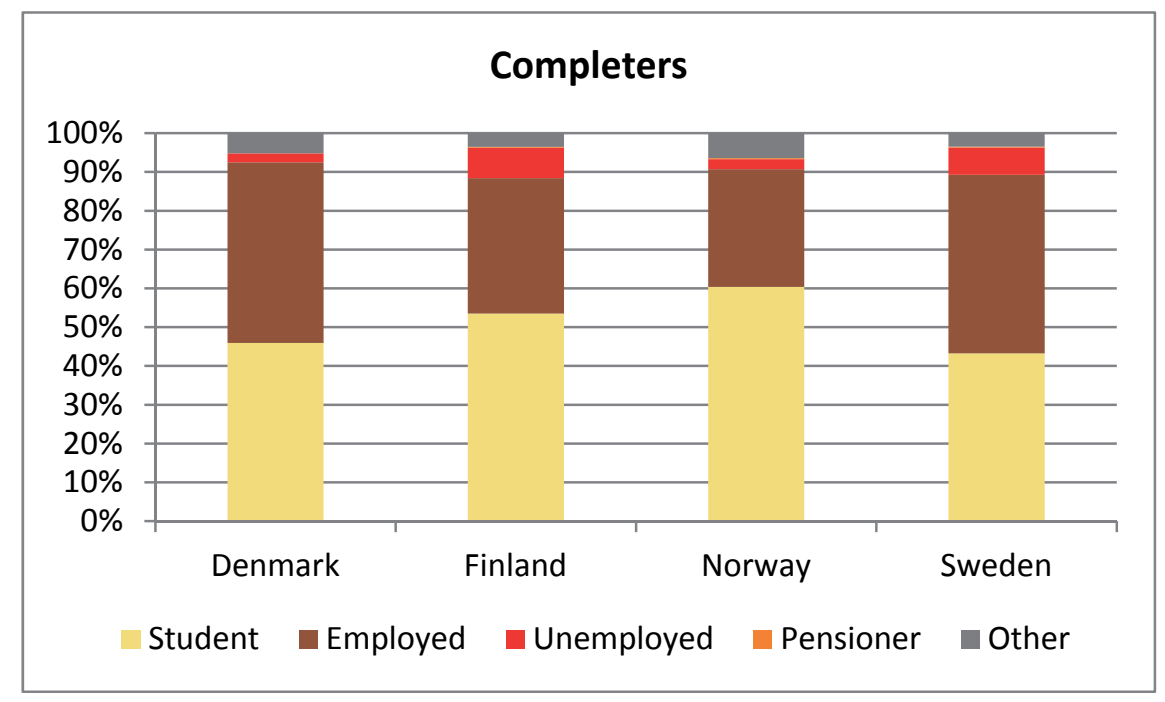




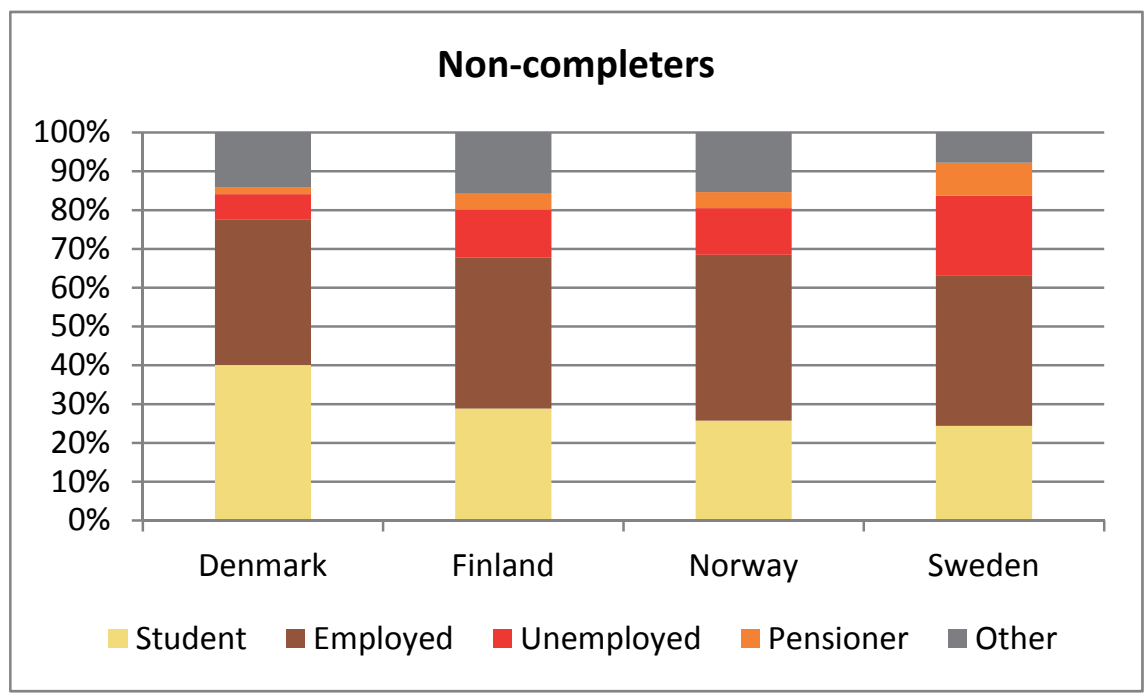

Notes: Completers are defined as young people having completed an upper secondary education by age 21 . Conversely, non-completers are defined as those having reached 21 years-of-age without finishing an upper secondary degree. The number of completers is 103,203 for Denmark, 158,611 for Finland, 109,723 for Norway and 243,763 for Sweden, whereas the number of non-completers is 61,676 for Denmark, 34,956 for Finland, 46,441 for Norway and 46,494 for Sweden. For definitions of the five main activity groups, see Chapter 2 .

These highly different cross-country patterns when it comes to both completers' and non-completers' shares in education and employment when aged 21 also explain the huge variation observed across the four countries when contrasting the "activity" (education + employment) share of completers to that of non-completers. As shown in Table 6.1, most of this "activity" gap originates in a dramatic cross-country variation in the gap between completers' and non-completers' enrolment in education: the difference in the share of 21-year-old completers and non-completers continuing in education is conspicuously large for Norway (an almost 35\%-point lower share for the non-completers) compared to a gap of less than 6\%-points for Denmark. The corresponding gap in employment shares is typically much smaller (about 9\%-points in Denmark and 7\%-points in Sweden) or even reversed. Indeed, the employment share is higher for 21-year-old non-completers than for their completer peers in Finland (about 4\%-points higher) and especially in Norway (about 12\%-points higher). 


\begin{tabular}{|c|c|c|c|c|c|c|c|c|}
\hline \multirow[t]{2}{*}{ Main activity } & \multicolumn{2}{|c|}{ Denmark } & \multicolumn{2}{|c|}{ Finland } & \multicolumn{2}{|c|}{ Norway } & \multicolumn{2}{|c|}{ Sweden } \\
\hline & $\begin{array}{c}\%- \\
\text { share }\end{array}$ & $\begin{array}{r}\begin{array}{r}\% \text {-point } \\
\text { gap }\end{array}\end{array}$ & $\begin{array}{c}\%- \\
\text { share }\end{array}$ & $\begin{array}{r}\text { \%-point } \\
\text { gap }\end{array}$ & $\begin{array}{c}\%- \\
\text { share }\end{array}$ & $\begin{array}{r}\text { \%-point } \\
\text { gap }\end{array}$ & $\begin{array}{c}\%- \\
\text { share }\end{array}$ & $\begin{array}{r}\text { \%-point } \\
\text { gap }\end{array}$ \\
\hline Student & 45.9 & -5.8 & 53.5 & -24.6 & 60.4 & -34.6 & 43.2 & -18.8 \\
\hline Employed & 46.6 & -9.1 & 34.9 & +4.1 & 30.3 & +12.4 & 46.0 & -7.1 \\
\hline Student + employed & 92.5 & -14.9 & 88.4 & -20.5 & 90.7 & -22.2 & 89.2 & -25.9 \\
\hline Unemployed & 2.3 & +4.3 & 7.8 & +4.5 & 2.6 & +9.4 & 7.0 & +13.5 \\
\hline Disability benefit (pensioner) & 0.0 & +1.8 & 0.3 & +3.8 & 0.2 & +3.9 & 0.2 & +8.3 \\
\hline Other (inactivity) & 5.2 & +8.9 & 3.5 & +12.3 & 6.5 & +8.9 & 3.5 & +4.3 \\
\hline NEET activities & 7.5 & +15.0 & 11.6 & +20.6 & 9.3 & +22.2 & 10.7 & +26.1 \\
\hline Total & 100.0 & & 100.0 & & 100.0 & & 100.0 & \\
\hline
\end{tabular}

Notes: These calculations are based on the distributions displayed in Figure 6.7. The \%-shares in the table show the distribution of completers across main activities when aged 21 (these shares are identical to those displayed in the upper graph of Figure 6.7). The \%-point gaps indicate the difference between non-completers' and completers' shares. A negative sign implies that the non-completers' share is lower: for instance, the share of Danish non-completers in education is $5.8 \%$-points lower (or $40.1 \%$ ) than the corresponding share for completers (45.9\%). Likewise, a positive sign means that a larger share of the non-completers than of the completers is in that particular activity.

The other side of the coin illustrates completers' and non-completers' involvement in various NEET activities. The share of completers showing up in such activities when aged 21 is small in all four countries, which follows directly from most of them being either full-time students or employed. Of the non-completers, on the other hand, about one-third is neither studying nor working by age 21 . Only in Denmark is this share smaller, under one-fourth. Moreover, a majority of the non-completers in NEET activities belongs to the dumping category of "other" (inactivity), that is, they do not appear in any of the large administrative registers from which our national datasets are compiled. A conspicuous exception from this pattern is Swedish non-completers, though, whose NEET activities are dominated by registered unemployment. Notable shares of the non-completers are also on disability benefits, a labour market outcome that is almost totally missing among 21-year-old completers in all four countries.

In view of these findings, it is hardly surprising that a comparison by country of completers' and non-completers' NEET activities when aged 21 results in a situation where the non-completers' shares throughout exceed those of the completers. However, the cross-country variation in also these patterns is substantial (Table 6.1). For instance, the share of the unemployed is quite high and of a similar size (about 7\%) among Finnish and Swedish completers aged 21. The share of unemployed noncompleters is, however, even larger $(12.3 \%$ in Finland and $20.5 \%$ in Sweden). This results in a remarkably large gap (13.5\%-points) in Swe- 
dish completers' and non-completers' unemployment shares, with the corresponding gap being notably lower (4.5\%-points) for Finland. A similar situation prevails for Denmark and Norway, where the share of 21-year-old completers in unemployment is very low (less than $3 \%$ ) while their non-completer peers experience either somewhat higher unemployment (Denmark) or much higher unemployment (Norway). As is evident in Table 6.1, similar examples can be found with respect to disability benefits, as well as other types of inactivity.

\subsubsection{Situation ten years later, at age 26}

Next we pick up these completers and non-completers at age 26. As noted above, we thereby retain the classification of our young people into completers and non-completers that prevailed when they turned 21. This exercise results in the country-specific distributions across main activities shown in the two graphs contained in Figure 6.8.

Among the completers, employment has by age 26 taken over as the overwhelmingly most common activity, while their share in full-time education has shrunk to between one-third (Denmark) and one-fourth (Sweden). Taken together, the share of completers either studying or working is at this age slightly higher than five years earlier, at age 21, but only for Denmark and Sweden. For Finnish completers, the "activity" share stands at approximately the same level as five years earlier, obviously mainly due to the conspicuous share outside both education and the labour market observed among young women at age 26 (and also at age 31) in the previous sub-chapter. For Norway, in contrast, the share of completers either studying or working is slightly lower at age 26 than at age 21. This finding is in line with the observations made earlier in this chapter, viz. a decline in the share of students that exceeds the growth in the share in employment, coupled with a concomitant increase in the share of young people in inactivity (cf. Tables 6.1 and 6.2).

Also the share of non-completers in either education or employment is higher at age 26 than at age 21: marginally higher in Denmark (78 vs. $79 \%$ ) and Norway (69 vs. 70\%), but notably higher in Sweden (63 vs. $71 \%$ ). Only for Finland do we see a decline in non-completers' "activity" share over these five years, just as for Finnish completers and, evidently, for the same main reason. In all four countries, the "activity" share of noncompleters lags, nonetheless, far behind that of completers still at age 26 (Table 6.2). However, the cross-country variation in this "activity" gap between completers and non-completers is much smaller at age 26 than at age 21 . In particular, while this activity gap between completers and non- 
completers has remained approximately unchanged from age 21 up to age 26 in Denmark, it has narrowed quite substantially in Sweden (cf. Tables 7.1 and 7.2). Yet, the way in which the gaps between completers and noncompleters in student and employment shares feed into this "activity" gap looks totally different at age 26 , when compared five years earlier. Noteworthy is especially the change in the employment gap: by age 26 , it has turned from positive to negative (Finland and Norway) or even more negative (Sweden). At age 26, non-completers thus typically face a weaker (Norway) or much weaker (Finland and Sweden) employment situation than completers, except in Denmark (Table 6.2).

The share of 21-year-old completers outside education and employment was noted to be low in all four countries. The share of completers in NEET activities has declined further by age 26 . Among non-completers of age 21, large shares were found to be either unemployed, on disability benefits or in other types of inactivity, with this share ranging from about $22 \%$ in Denmark up to almost 37\% in Sweden. The situation has, by age 26, improved slightly among Swedish non-completers but only marginally so among Danish and Norwegian non-completers. For Finnish non-completers, the NEET share is basically unchanged and now, in effect, highest among the four countries. However, just as the "activity" gap, also the NEET gap is constructed in a different way at age 26 than age 21 . Put differently, the gaps in completers' and non-completers' shares in unemployment, disability arrangements and other inactivity feed into the total NEET gap in highly different ways at age 21 and at age 26 (cf. Tables 6.1 and 6.2).

On the whole, though, the overall cross-country pattern in relation to completers' and non-completers' distributions across main activities does not change that much when comparing the situation at age 26 to the situation prevailing at age 21 . The highest "activity" share is still observed for Denmark, and this holds true for both completers and non-completers. The ranking of the other three countries has changed, though, with Sweden surpassing both Finland and Norway with respect to both completers and noncompleters. Then follows Norway and, finally, comes Finland. Indeed, at age 26, Finland comes out with the lowest "activity" share and, conversely, the highest NEET share among both completers and non-completers. As noted above, this outcome for Finland seems to be due, at least in part, to the growing share beyond age 21 of young women outside both education and the labour market. Additionally, (registered) unemployment turns out to be quite widespread among Finnish non-completers aged 26. However, as has been indicated in earlier parts of this report and as will be shown later on, this is largely a cohort effect related to the weak employment prospects faced by young labour market entrants in the 1990s. 
Figure 6.8: Main activities at age 26 for completers and non-completers of an upper secondary education, based on pooled information on two (1993 and 1998) of the youth cohorts under study, by country
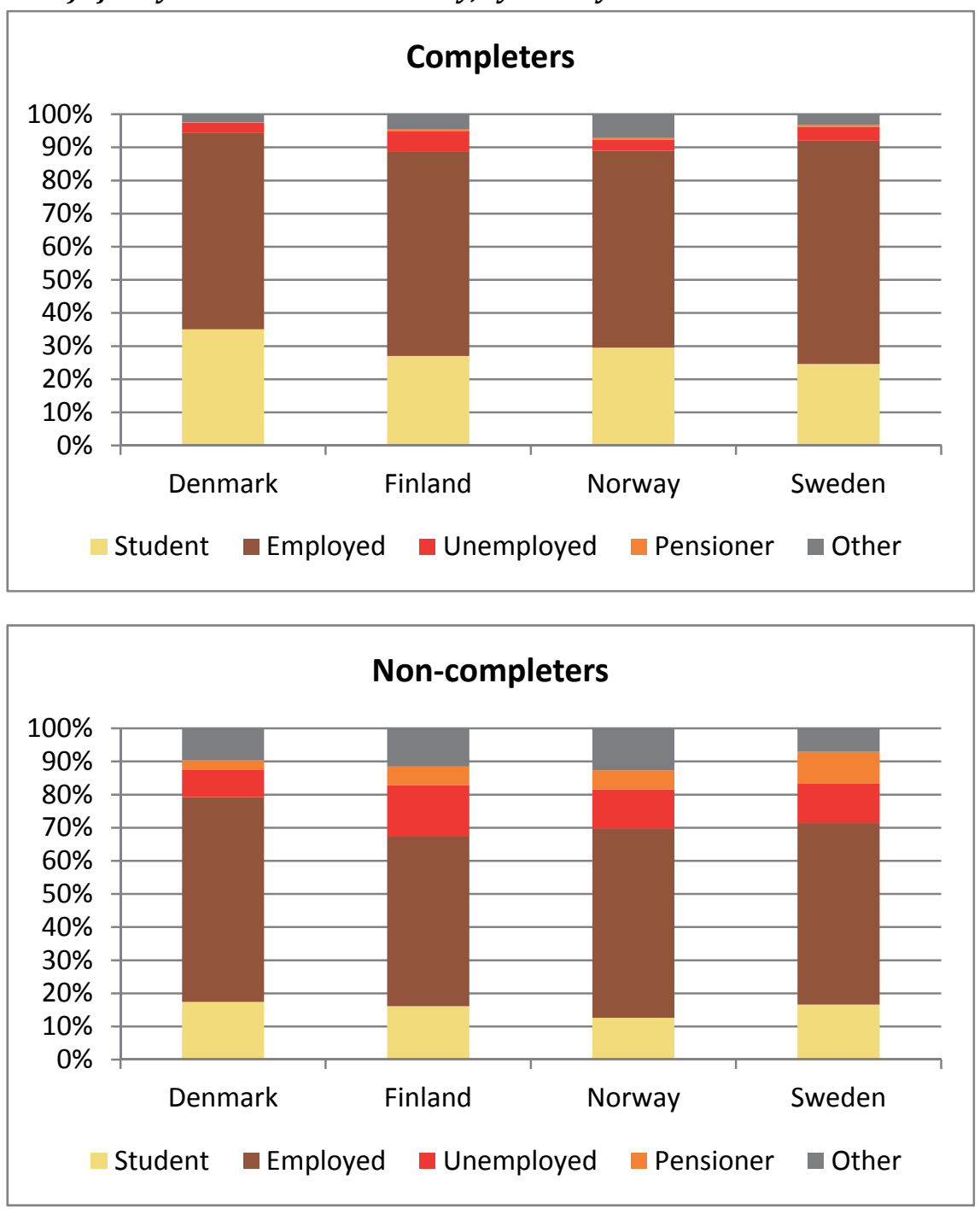

Notes: Completers are defined as young people having completed an upper secondary education by age 21 . Conversely, non-completers are defined as those having reached 21 years-of-age without finishing an upper secondary degree. Recall that we are not able to trace the 2003 cohort of 16 -year-olds up to age 26 , only up to age 21 . This also affects the total number of completers and non-completers in respective country underlying the \%-shares displayed in this particular figure: now the number of completers is 68,003 for Denmark, 108,935 for Finland, 72,885 for Norway and 154,288 for Sweden, whereas the number of non-completers is 36,762 for Denmark, 23,728 for Finland, 29,521 for Norway and 28,818 for Sweden. For definitions of the five main activity groups, see Chapter 2. 


\begin{tabular}{|c|c|c|c|c|c|c|c|c|}
\hline \multirow[t]{2}{*}{ Main activity } & \multicolumn{2}{|c|}{ Denmark } & \multicolumn{2}{|c|}{ Finland } & \multicolumn{2}{|c|}{ Norway } & \multicolumn{2}{|c|}{ Sweden } \\
\hline & $\begin{array}{r}\%- \\
\text { share }\end{array}$ & $\begin{array}{r}\text { \%-point } \\
\text { gap }\end{array}$ & $\begin{array}{c}\text { \%- } \\
\text { share }\end{array}$ & $\begin{array}{r}\text { \%-point } \\
\text { gap }\end{array}$ & $\begin{array}{r}\text { \%- } \\
\text { share }\end{array}$ & $\begin{array}{r}\text { \%-point } \\
\text { gap }\end{array}$ & $\begin{array}{c}\%- \\
\text { share }\end{array}$ & $\begin{array}{r}\text { \%-point } \\
\text { gap }\end{array}$ \\
\hline Student & 35.1 & -17.7 & 27.0 & -10.9 & 29.5 & -16.9 & 24.6 & -8.0 \\
\hline Employed & 59.2 & +2.7 & 61.6 & -10.2 & 59.4 & -2.2 & 67.3 & -12.5 \\
\hline Student + employed & 94.3 & -15.0 & 88.6 & -21.1 & 88.9 & -19.1 & 91.9 & -20.5 \\
\hline Unemployed & 3.2 & +4.9 & 6.1 & +9.2 & 3.3 & +8.5 & 4.2 & +7.8 \\
\hline Disability benefit (pensioner) & 0.1 & +2.9 & 0.6 & +5.0 & 0.5 & +5.3 & 0.6 & +8.9 \\
\hline Other (inactivity) & 2.4 & +7.2 & 4.6 & +6.9 & 7.2 & +5.5 & 3.2 & +3.9 \\
\hline NEET activities & 5.7 & +15.0 & 11.3 & +21.1 & 11.0 & +19.3 & 8.0 & +20.6 \\
\hline Total & 100.0 & & 100.0 & & 100.0 & & 100.0 & \\
\hline
\end{tabular}

Notes: These calculations are based on the distributions displayed in Figure 6.8. The \%-shares in the table show the distribution of completers across main activities when aged 26 (these shares are identical to those displayed in the upper graph of Figure 6.8). The \%-point gaps indicate the difference between non-completers' and completers' shares. A negative sign implies that the non-completers' share is lower: for instance, the share of Danish non-completers in education is $17.7 \%$-points lower (or $17.4 \%$ ) than the corresponding share for completers (35.1\%). Likewise, a positive sign means that the share of the non-completers is larger than that of the completers in that particular activity.

\subsubsection{Situation 15 years later, at age 31}

Finally we compare the labour market outcomes of completers and noncompleters at age 31 , that is, 15 years after these young adults left compulsory education. At this particular age, about $95 \%$ of the Danish and $94 \%$ of the Swedish completers are either employed or enrolled in education (Figure 6.9, upper graph), that is, a still larger share than at age 26. In Finland, this "activity" share is $89 \%$, which corresponds to an only marginally higher share than five years earlier. In Norway, on the other hand, this share has declined slightly, from $89 \%$ at age 26 to below $87 \%$ at age 31 , a pattern observed also in earlier parts of this report.

Of the Danish and Swedish completers, about $85 \%$ are in working life when aged 31, compared to some $76 \%$ of Finnish and Norwegian completers. Hence, the completers' employment situation has improved further by age 31.The share of completers still enrolled as full-time students has shrunk to below 10\% in Denmark and Sweden and to less than $11 \%$ in Norway. The highest share is found for Finland, or 13.5\%. 
While the employment situation has, in all four countries, improved also for the non-completers over the 5-year period from age 26 to age 31 , the growth in the non-completers' employment share has been clearly weaker: up to a share of $73 \%$ in Denmark, $65 \%$ in Sweden, $62 \%$ in Norway and $60 \%$ in Finland (lower graph of Figure 6.9). As a consequence, the gap in employment shares between completers and noncompleters is in all four countries larger at age 31 than at age 26 (cf. Table 6.2 and 6.3). Simultaneously, the gap in completers' and noncompleters' student shares has turned small (Finland and Norway) or negligible (Denmark and Sweden). This also explains why the "activity" gap between completers and non-completers is, in effect, slightly smaller at age 31 than at age 26, except for Sweden where it is unchanged and, hence, still highest among the four countries (Table 6.3). However, despite these various trends between and within countries up to age 31, the overall cross-country pattern observed for completers and noncompleters at age 26 shows up also five years later, at age 31: an employment share and, hence, also an "activity" share of non-completers lagging far behind that of completers.

Figure 6.9: Main activities at age 31 for completers and non-completers of an upper secondary education, based on information on one (1993) of the youth cohorts under study, by country

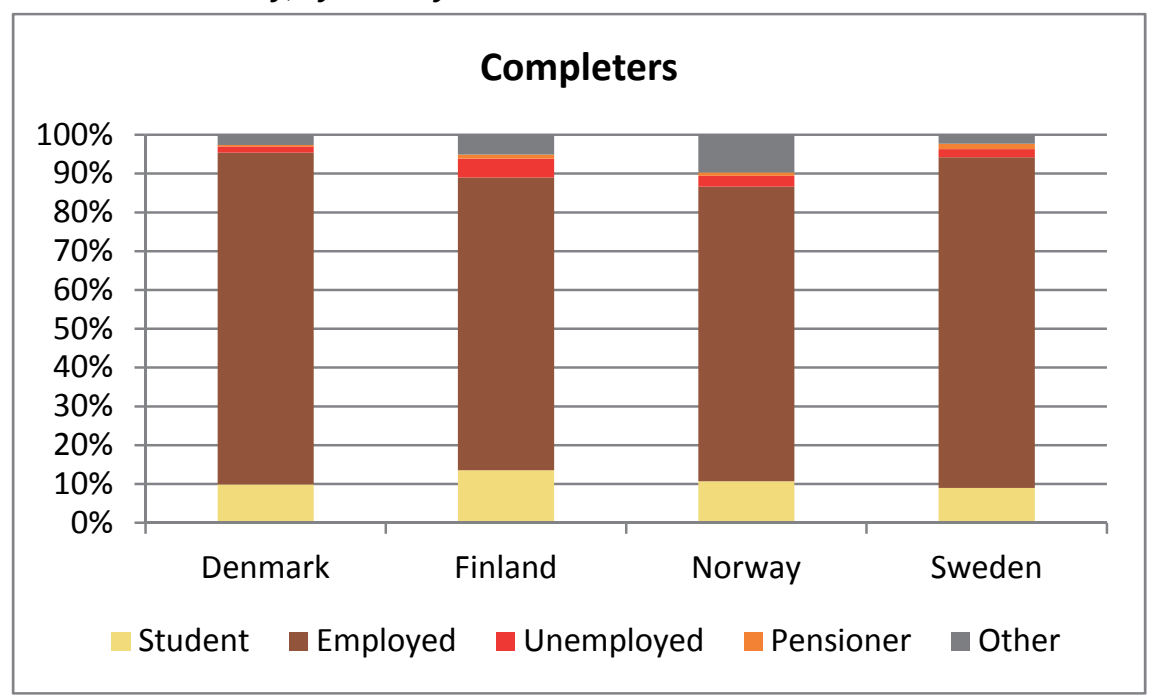




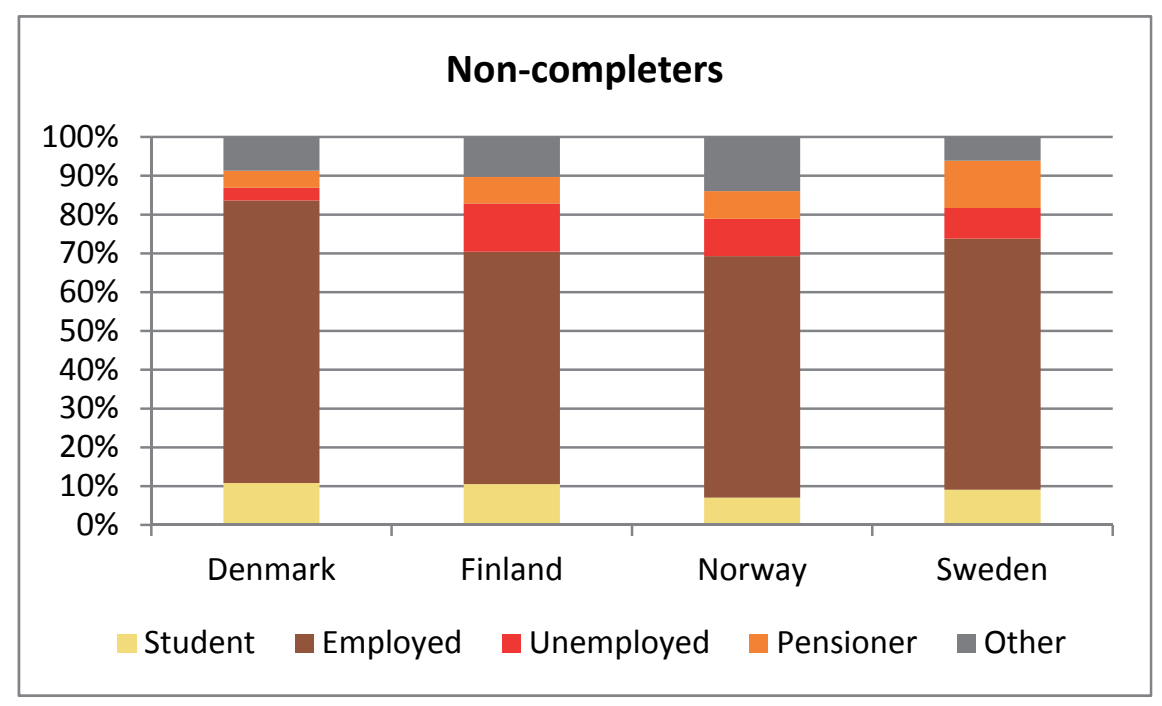

Notes: Completers are defined as young people having completed an upper secondary education by age 21. Conversely, non-completers are defined as those having reached 21 years-of-age without finishing an upper secondary degree. Recall that we are not able to trace the 1998 and 2003 cohorts of 16-year-olds up to age 31. This affects the total number of completers and non-completers in the respective country underlying the \%-shares displayed in this particular figure: now the number of completers is 36,932 for Denmark, 55,088 for Finland, 36,451 for Norway and 77,498 for Sweden, whereas the number of non-completers is 19,778 for Denmark, 10,507 for Finland, 14,561 for Norway and 13,113 for Sweden. For definitions of the five main activity groups, see Chapter 2 .

The steady increase up to age 31 in the share of Danish and Swedish completers in either education or employment is mirrored by a concomitant decrease in their share in NEET activities. Indeed, the share of 31year-old completers outside both education and work is very low in Denmark and Sweden. In Finland, it is about 11\% among both 26-yearold and 31-year-old completers, with unemployment showing up as an important explanation for also 31-year-old completers' comparatively high NEET share. In Norway, in contrast, the share of completers in NEET activities is slightly higher at age $31(13 \%)$ than at age $26(11 \%)$. This relatively large and increasing NEET share among Norwegian completers seems to be mainly due to withdrawal not only from education but also from the labour market: the share of completers in unknown inactivity increases steadily from age $21(6.5 \%)$ up to at age 31 (close to $10 \%$ ). While a similar trend is discernible also for those receiving disability benefits, the share of disability beneficiaries among Norwegian completers is still by age 31 below one per cent. 


\begin{tabular}{|c|c|c|c|c|c|c|c|c|}
\hline \multirow[t]{2}{*}{ Main activity } & \multicolumn{2}{|c|}{ Denmark } & \multicolumn{2}{|c|}{ Finland } & \multicolumn{2}{|c|}{ Norway } & \multicolumn{2}{|c|}{ Sweden } \\
\hline & $\begin{array}{r}\%- \\
\text { share }\end{array}$ & $\begin{array}{r}\text { \%-point } \\
\text { gap }\end{array}$ & $\begin{array}{r}\%- \\
\text { share }\end{array}$ & $\begin{array}{r}\text { \%-point } \\
\text { gap }\end{array}$ & $\begin{array}{c}\%- \\
\text { share }\end{array}$ & $\begin{array}{r}\text { \%-point } \\
\text { gap }\end{array}$ & $\begin{array}{c}\%- \\
\text { share }\end{array}$ & $\begin{array}{r}\text { \%-point } \\
\text { gap }\end{array}$ \\
\hline Student & 9.9 & +0.9 & 13.5 & -2.9 & 10.7 & -3.6 & 9.0 & +0.1 \\
\hline Employed & 85.5 & -12.6 & 75.5 & -15.6 & 76.0 & -13.8 & 85.3 & -20.6 \\
\hline Student + employed & 95.4 & -11.7 & 89.0 & -18.5 & 86.7 & -17.4 & 94.3 & -20.5 \\
\hline Unemployed & 1.6 & +1.7 & 4.8 & +7.7 & 2.8 & +6.8 & 2.1 & +5.8 \\
\hline Disability benefit (pensioner) & 0.4 & +4.0 & 1.1 & +5.8 & 0.9 & +6.2 & 1.4 & +10.8 \\
\hline Other (inactivity) & 2.6 & +6.1 & 5.1 & +5.1 & 9.7 & +4.3 & 2.3 & +3.8 \\
\hline NEET activities & 4.6 & +11.8 & 11.0 & +18.6 & 13.4 & +17.3 & 5.8 & +20.4 \\
\hline Total & 100.0 & & 100.0 & & 100.0 & & 100.0 & \\
\hline
\end{tabular}

Notes: These calculations are based on the distributions displayed in Figure 6.9. The \%-shares in the table show the distribution of completers across main activities when aged 31 (these shares are identical to those displayed in the upper graph of Figure 6.9). The \%-point gaps indicate the difference between non-completers' and completers' shares. A negative sign implies that the non-completers' share is lower: for instance, the share of Danish non-completers in employment is $12.6 \%$-points lower (or $72.9 \%$ ) than the corresponding share for completers (85.5\%). Likewise, a positive sign means that a larger share of the non-completers than of the completers is in that particular activity.

Denmark shows up with the lowest NEET share (16.4\%) also among 31year-old non-completers, whereas substantially higher shares emerge for the other three countries: about $26 \%$ for Sweden, close to $30 \%$ for Finland and almost $31 \%$ for Norway. Hence, still by age 31 remarkably large shares of the non-completers stand outside both education and employment. Indeed, their NEET share is only marginally lower than five years earlier, at age 26, and in Norway it reveals an increasing rather than decreasing trend. The moderate change in the non-completers' NEET share in adulthood is mainly explained by two opposite trends: declining shares of non-completers in unemployment (compared with the situation at age 26) and growing shares of them withdrawing from the labour market, especially into disability arrangements. Accordingly it is not surprising that the gap in the share of completers and noncompleters on disability benefits has increased in all four countries from age 26 up to age 31, whereas the corresponding gaps in unemployment and inactivity shares have typically declined over these five years.

\subsubsection{The influence of economic shocks}

As a final exercise of this sub-chapter, we again split the information provided at age 26 into two parts: one illustrating the distribution across main activities at this particular age of young people belonging to the "economic-bust" cohort of 1993, and one providing the same information for the "economic-boom" cohort of 1998. Needless to say, the 
focus now is on a comparison by cohort of the situation of completers vs. that of non-completers. The outcome of this exercise is presented in Figure 6.10, separately for each of the four Nordic countries under study.

The first graph of Figure 6.10 gives the distribution across main activities of Danish completers and non-completers belonging to respective youth cohort. The "activity" (education + employment) share of the 1998 economic-boom cohort is slightly higher than for the 1993 economic-bust cohort among both completers and non-completers. Conversely, the 1993 cohort is characterised by a somewhat higher NEET share irrespective of whether or not the young person has completed an upper secondary degree by age 21 . In this sense, the 1998 economicboom cohort seems to have experienced a more favourable labour market situation when aged 26. Simultaneously, however, the changes across NEET activities have been remarkable, and not necessarily always to the favour of the 1998 cohort. In particular, while the share in registered unemployment is smaller (completers) or dramatically smaller (non-completers) in the 1998 cohort, when compared to the 1993 cohort, the situation is reversed when it comes to time spent outside both education and the labour force: higher shares of young people belonging to the 1998 cohort are, when aged 26, either on disability benefits or in other (unknown) types of inactivity. Moreover, this holds true for both completers and non-completers. These findings point to a "reshuffling" of NEETs rather than to a straightforward impact of an economic shock.

The next graph provides the corresponding information for Finland. As for Denmark, we see a clear improvement of completers' and especially of non-completers' engagement in education and employment across the two cohort, and a corresponding reduction in their NEET activities. But in contrast to Denmark, the shares among both completers and non-completers in disability arrangements or other types of inactivity are basically the same in the two cohorts. In other words, the decline in NEET activities across the two cohorts is in Finland almost entirely explained by less registered unemployment in the 1998 economic-boom cohort than in the 1993 economic-bust cohort. These findings are well in line with the high unemployment levels that prevailed long after the start of the economic recovery in 1994. 
Figure 6.10: Distribution (\%-share) of completers and non-completers of an upper secondary education across main activities at age 26, by country; comparison of the "economic-bust" cohort of 1993 with the "economic-boom" cohort of 1998
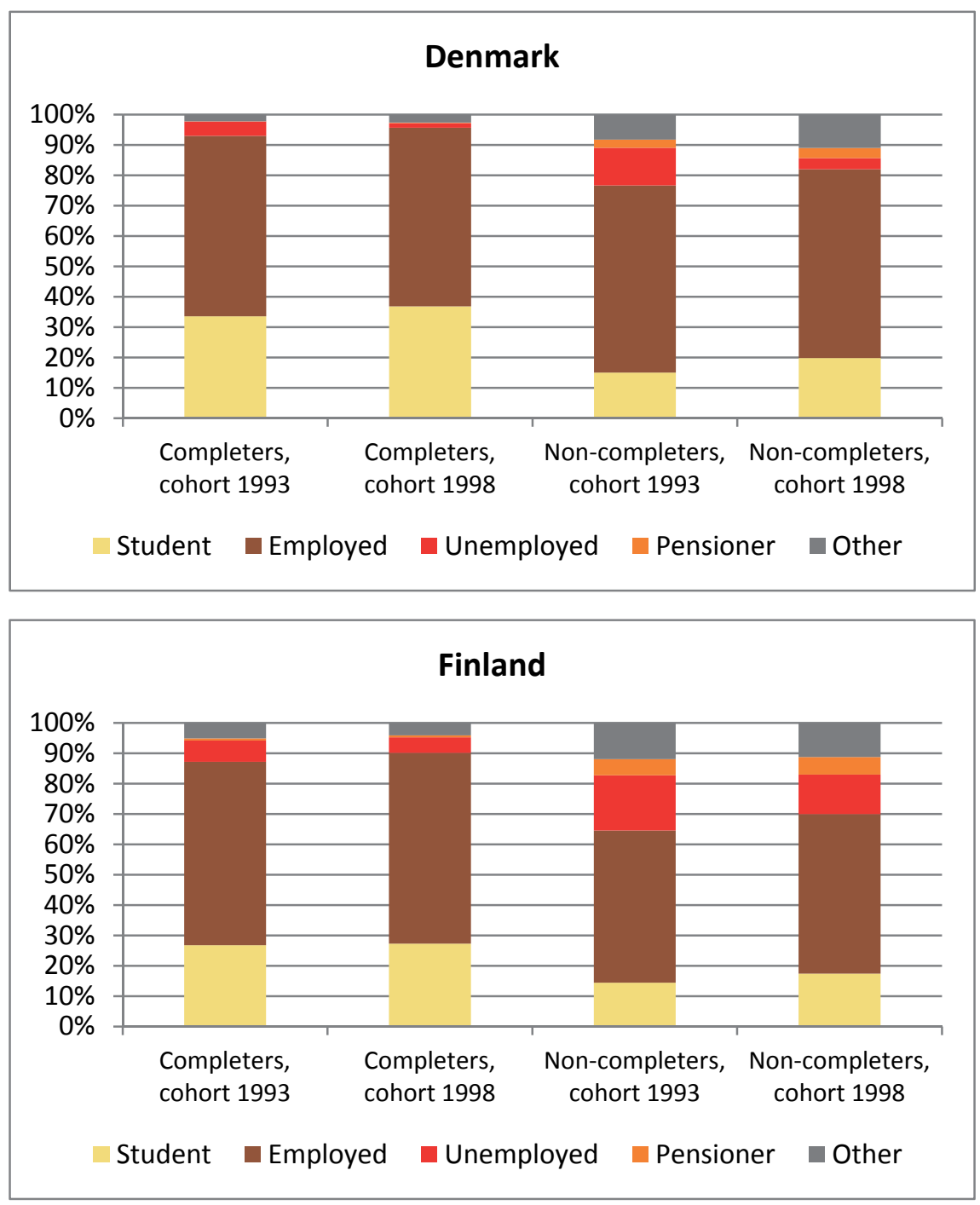

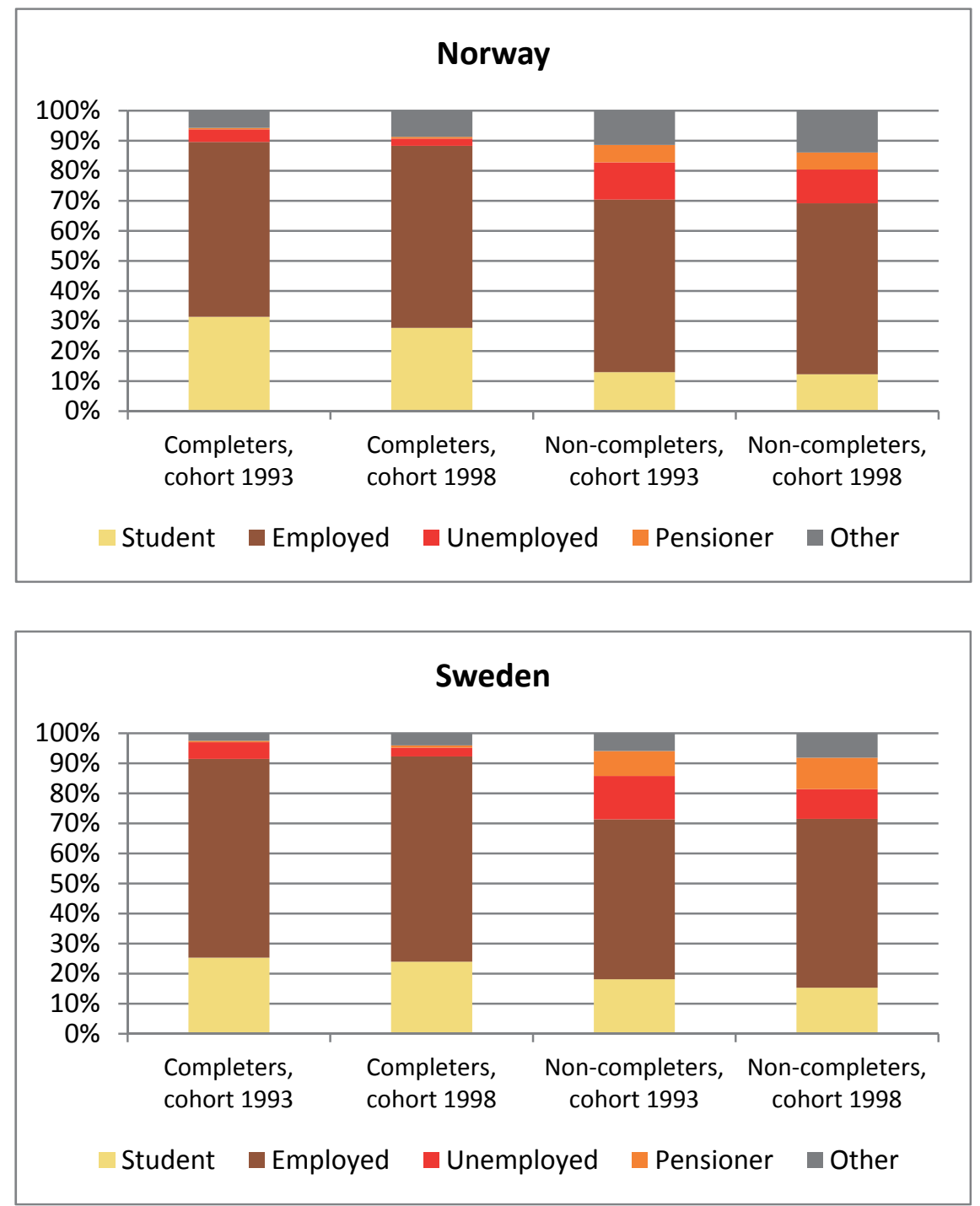

Notes: See Figure 6.7.

The graph displaying the situation for Norwegian completers and noncompleters belonging to either one of the two cohorts repeats a pattern that has already been discernible in previous parts of this report: a drop in the share of students that is not fully compensated by the concomitant increase in employment, resulting in an "activity" (education + employment) share that reveals a declining rather than increasing trend over cohorts (as well as within cohorts with age). Simultaneously, we see more young people moving outside both education and the labour force. This trend shows up strongly among both completers and non-completers. 
The last graph of Figure 6.10 illustrates the situation for Sweden. The combined education and employment share is slightly higher for completers belonging to the 1998 economic-boom cohort, but among noncompleters it is of approximately of the same size in the two cohorts. Hence, while the completers' NEET share is slightly lower in the 1998 cohort, it is unchanged across the two cohorts for non-completers. As in Denmark and Norway, much more seems to happen with the composition of NEETs: lower shares of unemployed jobseekers in the 1998 cohort but at the expense of higher shares outside both education and the labour force. Again, the same pattern shows up among both completers and non-completers.

All in all, the most profound difference between completers and noncompleters is much weaker employment prospects of non-completers and, hence, notably higher risks of ending up in NEET activities. Economic business cycles, on the other hand, seem to impact on young people in much the same way irrespective of their educational background (completion or not of an upper secondary degree by age 21). But with an initially weaker labour market attachment of non-completers, it is not surprising that also the consequences of an economic shock are typically conceived to be more serious for low-skilled young people. Apart from a persistently higher NEET share among non-completers, another conspicuous trend that seems to be little, if at all, linked to the prevailing economic situation is the growing tendency among non-completers of moving into potentially more risky NEET activities, that is, withdrawal not only from education but also from the labour force, including registered unemployment. In Norway, this trend is for some reason conspicuously common and increasing also among completers.

\subsubsection{Main findings}

The main finding is, unsurprisingly, that lower shares of non-completers than of completers are either in education or in employment and that this gap prevails up to age 31, at least. In other words, non-completers do not close up the gap to completers in their "activity" (education + employment) share. Simultaneously, however, this gap to completers in terms of education and employment seems to be relatively small, or at least smaller than would perhaps have been expected in view of the discussion of lowskilled young people being the losers in today's labour markets.

Indeed, the conspicuous "success rates" in terms of studying and working observed for also non-completers could be taken to indicate that, in the last resort, many of them fare reasonably well in the labour 
market as young adults despite a low formal education. The highest success rates are obtained for Denmark. The difference in non-completer outcomes between Denmark, on the one hand, and Finland and Sweden, on the other hand, could then be interpreted as a result of notable crosscountry differences in the composition of the group of non-completers. The so-called hard core of non-completers, that is, young people with disproportionally weak labour market prospects, tend to drop out from education at an early age in principally any country. If the number of non-completers increases, this most likely implies that also young people with less serious problems and, hence, with an obviously closer labour market attachment, are for some reason shifting into the group of non-completers. If this is the case, then the overall size of the group of non-completers could also tell us something about the composition of non-completers. For this very reason we would expect the relatively large share of 21-year-old Danish non-completers to do better on average than the comparatively small shares of non-completers among the 21-year-olds observed for Finland and Sweden (cf. Chapter 2).

However, this interpretation does not get support when comparing Finland and Sweden to Norway. In this setting, a large cross-country difference in non-completion rates does not result in conspicuously different success rates of the three countries' non-completers. Moreover, even in the case of relatively high employment rates also among noncompleters, previous research has shown that there is a large and significantly negative wage differential between employed non-completers and employed completers (see Bratsberg et al., 2010).

Apart from differences in the quality of completers' and noncompleters' employment contracts, a dimension overlooked in our analyses, there are worrying aspects also related to the time trend in noncompleters' labour market prospects. In particular, the results presented in this sub-chapter point to the employment share of non-completers lagging increasingly behind that of completers when comparing their situation at three different age points $(21,26$ and 31$)$. This is highly evident in Table 6.4, which reproduces parts of the information provided in Tables 6.1 to 6.3 . Simultaneously, the cross-country variation in the gaps having emerged by age 31 between completers' and non-completers' employment shares is particularly pronounced when it comes to Denmark and Sweden: in both countries, completers fair equally well in terms of employment, whereas the gap to the non-completers' employment share is almost twice as large in Sweden (20.6\%-points) as it is in Denmark (12.6\%-points). 
Table 6.4: Selected main activities at age 21, 26 and 31, respectively: completers (\%-share) vs. non-completers (\%-point gap)

\begin{tabular}{lrrrrrrr} 
Main activity & \multicolumn{2}{c}{$\begin{array}{c}\text { Denmark } \\
\text { \%- }\end{array}$} & \multicolumn{2}{c}{ Finland } & \multicolumn{2}{c}{ Norway } & \multicolumn{2}{c}{ Sweden } \\
& $\begin{array}{r}\text { \%-point } \\
\text { share }\end{array}$ & $\begin{array}{r}\text { \%- } \\
\text { gap }\end{array}$ & $\begin{array}{r}\text { \%-point } \\
\text { share }\end{array}$ & $\begin{array}{r}\text { \%- } \\
\text { share }\end{array}$ & $\begin{array}{r}\text { \%-point } \\
\text { gap }\end{array}$ & $\begin{array}{r}\text { \%-point } \\
\text { share }\end{array}$ \\
gap
\end{tabular}

Notes: See Tables 6.1 to 6.3 .

The counterpart to this development is a steadily growing share of noncompleters moving outside both education and the labour market, either into disability arrangements or other (unknown) types of inactivity. While the share of disability beneficiaries increases with age also among young completers, this share is initially much higher and also increases much faster among the non-completers, a pattern repeated for all four countries.

When it comes to other types of inactivity, the situation varies substantially across countries, as also pointed out earlier. For all four countries, we observe a narrowing gap in completers' and non-completers' "other" inactivity shares (Table 6.4). However, in both Denmark and Sweden this is due to a decline with age in the inactivity share among both completers and non-completers, with this trend being stronger for the noncompleters. In Finland and especially in Norway, on the other hand, we see growing numbers of completers withdrawing from both education and the labour force when going from age 21 up to age 31, whereas an opposite trend is observable among non-completers. Indeed, the share in unknown inactivity among 31-year-old Norwegian completers is not only much higher than the corresponding share for completers in the other three countries. It also exceeds by far the inactivity share of both Danish and Swedish non-completers aged 31, while it is only marginally lower than the inactivity share of 31-year-old non-completers in Finland.

Moreover, these overall patterns and trends across and within the four Nordic countries under study, as well as across and within the three youth cohorts under scrutiny, seem to have been only marginally, if at all, affected by changes in the economic environment. Changing business cycles seem to typically impact on young people in much the same way 
irrespective of their upper secondary graduation background (completer vs. non-completer). However, with an initially much weaker labour market attachment, the consequences are inevitably more far-reaching for young non-completers than for their completer peers.

Finally, we undertake a rescaling of also these findings by relating them to the full youth population of each of the four Nordic countries under study. Table 6.5 displays the outcome of this exercise by main activity at age 21,26 and 31, respectively, separately for completers and non-completers.

About $50 \%$ of both Finnish and Norwegian youth are enrolled in fulltime education when aged 21, with completers contributing overwhelmingly to this high share. Sweden, in turn, comes out with the lowest share of students at this particular age mainly due to a very small share (under $4 \%$ ) of young non-completers continuing in education when aged 21. For Denmark, in contrast, we observe a strikingly high share of 21-yearold non-completers still in education. Conversely, Sweden turns out to have the highest share of 21-year-olds in employment, but this holds true for completers only. Young Swedes with only an exam from compulsory school still when aged 21 are the least likely of young Nordic non-completers of being in education or employment.

Table 6.5: Distribution of young completers and non-completers by main activity at age 21, 26 and 31 , respectively, by country, \%-share of the full youth population

\begin{tabular}{|c|c|c|c|c|c|c|c|c|}
\hline \multirow[b]{3}{*}{ Main activity } & \multicolumn{8}{|c|}{ Status at age $21-\%$-share of the full population } \\
\hline & \multicolumn{4}{|c|}{ Completers } & \multicolumn{4}{|c|}{ Non-completers } \\
\hline & DK & FI & NO & sw & DK & FI & NO & SW \\
\hline Student & 28.8 & 43.9 & 42.5 & 36.3 & 15.0 & 5.2 & 7.7 & 3.9 \\
\hline Employed & 29.2 & 28.6 & 21.3 & 38.6 & 14.0 & 7.0 & 12.7 & 6.2 \\
\hline Student + employed & 58.0 & 72.5 & 63.8 & 74.9 & 29.0 & 12.2 & 20.4 & 10.1 \\
\hline Unemployed & 1.4 & 6.4 & 1.8 & 5.9 & 2.5 & 2.2 & 3.6 & 3.3 \\
\hline Pensioner & 0.0 & 0.2 & 0.1 & 0.2 & 0.7 & 0.7 & 1.2 & 1.4 \\
\hline Other & 3.3 & 2.9 & 4.6 & 2.9 & 5.3 & 2.8 & 4.6 & 1.2 \\
\hline NEET share & 4.7 & 9.5 & 6.5 & 9.0 & 8.5 & 5.7 & 9.4 & 5.9 \\
\hline In total & 62.7 & 82.0 & 70.3 & 84.0 & 37.3 & 18.0 & 29.7 & 16.0 \\
\hline
\end{tabular}

\begin{tabular}{|c|c|c|c|c|c|c|c|c|}
\hline \multirow[b]{3}{*}{ Main activity } & \multicolumn{8}{|c|}{ Status at age $26-\%$-share of the full population } \\
\hline & \multicolumn{4}{|c|}{ Completers } & \multicolumn{4}{|c|}{ Non-completers } \\
\hline & DK & FI & NO & sw & DK & FI & NO & sw \\
\hline Student & 22.0 & 22.1 & 20.7 & 20.7 & 15.0 & 2.9 & 3.7 & 2.7 \\
\hline Employed & 37.1 & 50.5 & 41.8 & 56.5 & 14.0 & 9.3 & 17.0 & 8.8 \\
\hline Student + employment & 59.1 & 72.6 & 62.5 & 77.2 & 29.0 & 12.2 & 20.7 & 11.5 \\
\hline Unemployed & 2.0 & 5.0 & 2.3 & 3.5 & 2.5 & 2.8 & 3.5 & 1.9 \\
\hline Pensioner & 0.1 & 0.5 & 0.4 & 0.5 & 0.7 & 1.0 & 1.7 & 1.5 \\
\hline Other & 1.5 & 3.8 & 5.1 & 2.7 & 5.3 & 2.1 & 3.8 & 1.1 \\
\hline NEET share & 3.6 & 9.3 & 7.8 & 6.7 & 8.5 & 5.9 & 9.0 & 4.5 \\
\hline In total & 62.7 & 82.0 & 70.3 & 84.0 & 37.3 & 18.0 & 29.7 & 16.0 \\
\hline
\end{tabular}




\begin{tabular}{|c|c|c|c|c|c|c|c|c|}
\hline \multirow[b]{3}{*}{ Main activity } & \multicolumn{8}{|c|}{ Status at age $31-\%$-share of the full population } \\
\hline & \multicolumn{4}{|c|}{ Completers } & \multicolumn{4}{|c|}{ Non-completers } \\
\hline & DK & $\mathbf{F I}$ & NO & sw & DK & FI & NO & SW \\
\hline Student & 6.2 & 11.1 & 7.5 & 7.6 & 4.0 & 1.9 & 2.1 & 1.5 \\
\hline Employed & 53.6 & 61.9 & 53.4 & 71.7 & 27.2 & 10.8 & 18.5 & 10.4 \\
\hline Student + employed & 59.8 & 73.0 & 60.9 & 79.3 & 31.2 & 12.7 & 20.6 & 11.9 \\
\hline Unemployed & 1.0 & 3.9 & 2.0 & 1.8 & 1.2 & 2.3 & 2.9 & 1.3 \\
\hline Pensioner & 0.3 & 0.9 & 0.6 & 1.2 & 1.6 & 1.2 & 2.1 & 2.0 \\
\hline Other & 1.6 & 4.2 & 6.8 & 1.9 & 3.2 & 1.8 & 4.2 & 1.0 \\
\hline NEET share & 2.9 & 9.0 & 9.4 & 4.9 & 6.0 & 5.3 & 9.2 & 4.3 \\
\hline In total & 62.7 & 82.0 & 70.3 & 84.0 & 37.3 & 18.0 & 29.7 & 16.0 \\
\hline
\end{tabular}

Notes: See Tables 6.1 to 6.3 .

Sweden, together with Finland, also stands out with a comparatively high share of 21-year-olds registered as unemployed jobseekers, but only among young completers. Among 21-year-old non-completers, Norway shows up with the highest unemployment ratio, albeit the difference to the other three countries is rather small. However, together with Denmark, Norway also has a relatively high share of 21-year-olds whose activity is unknown. Moreover, this high inactivity share shows up among both young completers and young non-completers.

By age 26, Sweden still comes out with the highest employment share in the youth population but, again, only for completers; Swedish noncompleters' employment situation is weakest among Nordic noncompleters also at age 26. The share of young people enrolled in fulltime education still when aged 26 has, in turn, converged across the four countries and, moreover, among both completers and non-completers. The only exception is Denmark, where the share of non-completers continuing as full-time students is at the same high level as five years earlier, at age 21 .

When it comes to NEET activities, the overall cross-country pattern at age 26 is very similar to the situation observed at age 21 . In particular, the share of the unemployed has remained relatively high in both Finland and Sweden due to unemployment still being more common among Finnish and Swedish completers, when compared to Danish and Norwegian completers. And also among the 26-year-olds, Norway comes out with the highest share of unemployed jobseekers among the noncompleters. Also the share of young Norwegians outside both education and the labour market is comparatively high still at age 26 , with the inactivity share being even higher among completers than among noncompleters. While the inactivity share has remained at a high level also among Danish non-completers, the situation has improved among Danish completers. 
By age 31 , finally, all four countries have around $10 \%$ of their youths enrolled as full-time students, with the student share among the noncompleters now being very low. The employment share is high among Finnish and especially among Swedish completers, whereas the employment situation of the countries' non-completers is relatively weak still at age 31. A reversed situation is observed for Denmark and Norway in the sense that the employment share is comparatively low among completers but relatively high among non-completers.

The share of young people in unemployment has come down in all four countries and, moreover, among both completers and non-completers but is, nonetheless, still highest among Norwegian non-completers. Likewise, Norway has the highest share of young people outside both education and the labour market also among the 31-year-olds. Indeed, compared to the situation at age 26 , the inactivity share has increased rather than decreased among both completers and non-completers.

On the whole, then, the overall impression from this exercise of rescaling the main activity shares at three age points of completers and noncompleters in relation to the full youth population of each country, does not seem to change the general picture mediated so far. Particularly striking is the stability with age in the NEET share among the non-completers. Another conspicuous feature of Table 6.5 is the similarity in NEET shares across the four countries, a similarity that is higher than expected in view of the large differences observed in school-to-work-transition patterns and completing-rate time-profiles between the countries.

\subsection{Main activities beyond age 21 - late completion vs. non-completion}

We end this chapter on labour market outcomes in adulthood by relaxing our "prime" definition of non-completers. More precisely, we now allow for the fact that some of the non-completers, but far from all, do finalise an upper secondary education, but only after the age of 21, i.e., more than five years after having left compulsory school. As shown earlier, notably in Chapter 2, late completion of upper secondary education is common among young people in Denmark and Norway. In Finland and Sweden, on the other hand, a majority of young people has finalised an upper secondary education by the time they turn 21 .

We start this sub-chapter by exploring to what extent young people with only a basic education still at age 21 succeed in completing an upper secondary education later in life. Again, this is done for two age 
points: by the time they turn 26 and by the time they turn 31 . In the following, these young people are called "late completers" in order to separate them from 21-year-old completers, that is, young or early completers. Likewise, we use the term "adult non-completer" for young people lacking an upper secondary degree still at age 26 or age 31 in order to separate them from 21-year-old non-completers, that is, young noncompleters. In a next step, we compare the labour market outcomes of young completers, late completers and adult non-completers at these same age points.

This particular focus on late completers implies that most attention is paid to young people identified as non-completers at age 21. More precisely, the emphasis is on whether or not these 21-year-old noncompleters succeed in achieving an upper secondary certificate by age 26 or by age 31 , and to what extent this late completion eventually affects their labour market situation when compared to early completers and, especially, to adult non-completers. In other words, is early completion the best choice, late completion a second-best choice and noncompletion still in adulthood the worst alternative in terms of labour market outcomes as a young adult? This setting also means that all subsequent results are based on information on only two out of our three youth cohorts for the simple reason that the youngest cohort (the 2003 cohort of 16-year-olds) cannot be traced beyond the age of 21 .

\subsubsection{Late completion of upper secondary education}

There might be reason to first recall the shares of 21-year-old noncompleters in the four Nordic countries under study. We therefore start by reproducing the information provided in Table 2.2 of Chapter 2, but now with the youngest (2003) cohort of 16-year-olds left out. As has been pointed out earlier in this report, non-completion of an upper secondary degree is strikingly common among 21-year-old Danes, less so among 21-year-old Norwegians and quite infrequent among 21-year-old Finns and Swedes.

\begin{tabular}{|c|c|c|c|c|}
\hline Cohort & Denmark & Finland & Norway & Sweden \\
\hline 16-year-olds in 1993 & 34.7 & 16.0 & 28.5 & 14.5 \\
\hline 16-year-olds in 1998 & 39.0 & 19.7 & 29.1 & 17.0 \\
\hline
\end{tabular}

The next table (Table 6.7) shows to what extent these 21-year-old noncompleters have succeeded in finalising an upper secondary degree either by age 26 or by age 31 . In Denmark, more than one-half of the 21- 
year-old non-completers from the 1993 cohort had eventually completed an upper secondary degree by the time they turned 31 . Close to $42 \%$ of them had actually come around to completing an upper secondary education by age 26 . This high share of late completers also explains the rapid decline beyond age 21 in the non-completion share displayed in Figure 2.2a of Chapter 2 for the oldest (1993) Danish cohort of 16-yearolds. Moreover, this high share of late completers is repeated for the Danish 1998 cohort (about 43\% by age 26).

Table 6.7: Completion of an upper secondary degree by age 26 and by age 31 among 21-year-old non-completers, by country

\begin{tabular}{|c|c|c|c|c|c|c|c|c|}
\hline \multirow[b]{3}{*}{ Cohort } & \multicolumn{8}{|c|}{ Completion by age } \\
\hline & \multicolumn{2}{|c|}{ Denmark } & \multicolumn{2}{|c|}{ Finland } & \multicolumn{2}{|c|}{ Norway } & \multicolumn{2}{|c|}{ Sweden } \\
\hline & 26 & 31 & 26 & 31 & 26 & 31 & 26 & 31 \\
\hline 16-year-olds in 1993 & 41.6 & 51.8 & 25.4 & 34.7 & 26.3 & 36.3 & 19.3 & 26.4 \\
\hline 16-year-olds in 1998 & 42.9 & & 29.7 & & 29.1 & & 16.7 & \\
\hline
\end{tabular}

In the other three countries, the completion rates beyond age 21 are much lower. In Finland and Norway, slightly more than one-third of the young people in the 1993 cohort identified as non-completers still at age 21 had achieved an upper secondary certificate by the time they turned 31 with the corresponding share being only about $26 \%$ for Sweden. Moreover, while the late-completion rate reveals an increasing trend across cohorts in both Finland and Norway, it has rather been declining in Sweden.

These cross-Nordic differences in young non-completers' likelihood of achieving an upper secondary certificate only in adulthood are quite pronounced. It could be argued that the substantially lower noncompletion rates beyond age 21 observed for Finland, Norway and Sweden, when compared to Denmark, are related to the comparatively high share of non-completers among 21-year-old Danes (Table 6.6). However, the markedly higher non-completion share among 21-year-old Danes can only explain part of these conspicuous cross-country differences in late-completion rates. In particular, while the late-completion rates are very similar for Finland and Norway, the share of non-completers by age 21 is highly different in the two countries. Likewise, while the share of 21-year-old non-completers is of similar size in Finland and Sweden, there are substantial differences between the two countries when it comes to late-completion rates. In other words, there is no clear-cut cross-country correlation between the early- and late-completion rates reported in Tables 6.6 and 6.7. 


\subsubsection{Late completers: comparison of labour market outcomes at age 26}

The completion patterns of post-compulsory-school degrees displayed in Tables 6.6 and 6.7 raise several questions: Do early completers tend to fair better in terms of labour market outcomes than late completers? Likewise, do late completers typically fair better than adult noncompleters? The next figure aims to provide at least part of an answer to these questions with the focus being on the situation prevailing among 26-year-olds differing in their completion and non-completion history. In order to clean the picture from changing cohort-specific distributions across main activities as well as from business cycle fluctuations, Figure 6.11 is based on information for the 1998 cohort only, a cohort of young people that can be traced up to age 26 , but not to age 31 . We return to the situation among 31-year-olds in the next section.

Figure 6.11 contains four country-specific graphs, each of which displays three distributions across our five main activity categories. All three distributions refer to the situation prevailing at age 26 , whereas the allocation of these 26-year-olds across the three distributions depends on their completion and non-completion history with respect to post-compulsory-school educations. The first (left-hand-side) pillar illustrates the distribution across main activities of the 1998 cohort's young completers when aged 26 , that is, of those young people in the cohort who had completed an upper secondary education already by age 21. The second (middle pillar) shows the corresponding distribution for the cohort's late completers, that is, those young people in the cohort who were classified as non-completers when aged 21 but who had succeeded in completing an upper secondary degree by age 26 . The third (right-hand-side) pillar, finally, displays the distribution across main activities of the cohort's adult non-completers, that is, those young people in the cohort who still when aged 26 had an exam only from compulsory school.

The first graph of Figure 6.11 illustrates the situation for Danish young completers, late completers and adult non-completers belonging to the 1998 cohort. The differences in main-activity distributions between young completers and late completers are marginal at age 26 . In both groups, engagement in either education or employment is the overwhelmingly most common activity. Hence, the advantage of completing an upper secondary degree by age 21 instead of age 26 seems to be minor in Denmark, at least in terms of labour market outcomes in adulthood. The situation is entirely different for Danish adult noncompleters: an "activity" (education + employment) share of about $74 \%$ 
implies that one-fourth of the adult non-completers is in NEET activities when aged 26, belonging mainly to the dumping category of "other" (unknown) inactivity.

The next graph gives the corresponding information for Finland. It clearly shows that young completers fare much better than late completers. In particular, about nine out of ten young completers are engaged in either education or employment by age 26 . Among late completers, this share is approximately ten percentage points lower, or some $80 \%$. Instead, they face a higher risk of showing up as unemployed jobseekers or disability beneficiaries, when compared to young completers. However, compared to adult non-completers, these late completers do quite well. The "activity" share of adult non-completers is only about $65 \%$. While they have an only marginally higher risk of becoming unemployed, when compared to late completers, their risk of ending up in either disability arrangements or other types of inactivity is considerably higher than for late completers. Hence, early completion, late completion and non-completion still as a young adult makes a considerable difference in Finland.

A more or less similar overall pattern emerges for Norway except that the main-activity distributions of young and late completers are more similar in Norway than in Finland. Indeed, the difference in employment shares between young and late completers is marginal in Norway, whereas there are larger differences between the two groups when it comes to enrolment in education and registered unemployment: the share enrolled in education still at age 26 is higher among young completers, whereas the share in registered unemployment is higher among late completers. A conspicuous finding, however, is that the difference in inactivity shares among young and late completers is close to negligible, implying that young and late completers are equally likely to withdraw outside both education and the labour market. The distribution across main activities of adult non-completers is distinctly less favourable and, in effect, remarkably similar to that observed for adult non-completers in Finland: an "activity" share just above $60 \%$ in combination with large shares being in NEET activities already at age 26 . 
Figure 6.11: Main-activity distributions (\%-share) by age 26 of young completers vs. young non-completers having completed (late completer) or not having completed (adult non-completer) an upper secondary education by age 26, based on information for the 1998 youth cohort, by country
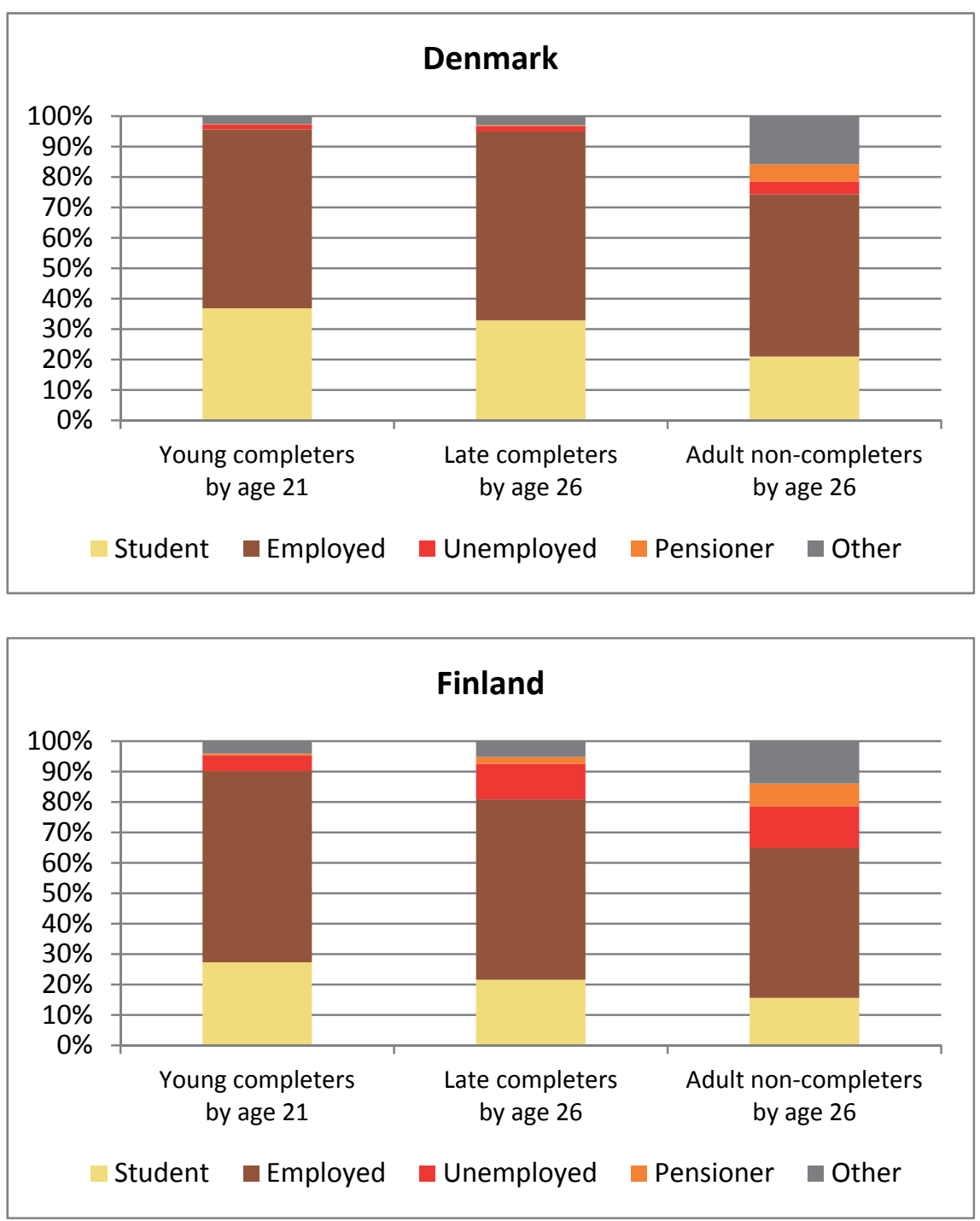

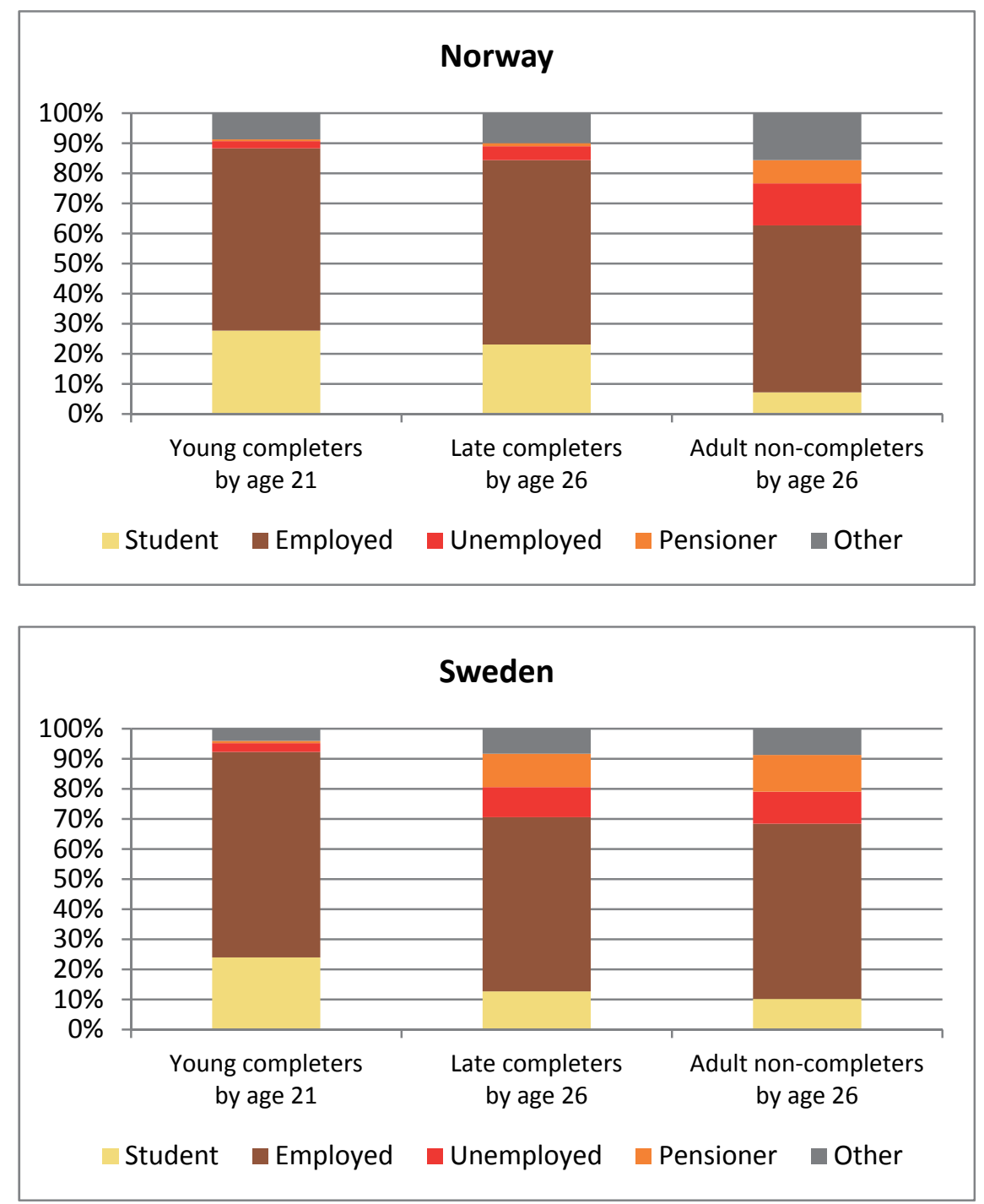

Notes: For definitions of the five main activity groups, see Chapter 2 . Young completers = young people having completed an upper secondary education already by age 21 . Late completers $=21-$ year-old non-completers having achieved an upper secondary degree by age 26 (but not yet by age 21). Adult non-completers = young people with only a basic education still at age 26 .

A third kind of pattern is discernible for Sweden (last graph of Figure 6.11). As noted earlier in this report, young Swedish completers fare extremely well: their "activity" (education + employment) share exceeds $90 \%$ by age 26 , a share that lags only slightly behind that of young Danish completers. Late completers, in contrast, do not seem to manage better in terms of labour market outcomes than do adult non-completers: the difference in main-activity distributions is strikingly small. While the "activity" share of 26 -year-old non-completers is about $68 \%$, it is only slightly 
higher for late completers. Hence, when compared to the other three countries, Swedish late completers fare worst, whereas Swedish adult non-completers fare better than both Finnish and Norwegian adult noncompleters, and only slightly worse than Danish adult non-completers.

\subsubsection{Late completers: comparison of labour market outcomes at age 31}

Next we compare the labour market outcomes of late completers and adult non-completers five years later, at age 31 (Figure 6.12). More precisely, we amend the setting used in the previous figure and add one more distribution: a pillar showing the distribution across main activities of young non-completers having achieved an upper secondary degree only by age 31 (but not yet by age 26). In other words, Figure 6.12 displays the activity distribution at age 31 for a total of four different categories of young people: those who had completed an upper secondary degree already by age 21 (young completer); those who had completed an upper secondary degree either by age 26 (late completers by 26) or by age 31 (late completer by 31); and those having an exam only from compulsory school still when aged 31 (adult non-completer). Consequently, all four distributions displayed in Figure 6.12 cover merely the 1993 cohort, as the two younger cohorts cannot be traced up to age 31 .

The first graph of Figure 6.12, displaying the situation at age 31 for young Danes differing in their completion and non-completion history, reveals several interesting results. First, the pattern of no clear-cut disadvantage from completing an upper secondary degree only by age 26 , instead of age 21, is repeated also for the 1993 cohort. Second, completion only by age 31 seems to have been an even better option, at least in terms of labour market outcomes. Third, adult non-completion, now at age 31, results in much the same outcome as adult non-completion at age 26: an "activity" share of about $74 \%$ and one-fourth of adult noncompleters in NEET activities. Finally, when comparing the results for young completers and late completers by age 26 with those displayed in Figure 6.11 for the 1998 cohort, the 1993 cohort seems to have fared slightly worse in terms of unemployment, which is in line with the results reported earlier in this chapter (when comparing the 1993 "economic-bust" cohort to the 1998 "economic-boom" cohort). The conspicuously good labour market performance of young people having graduated from upper secondary education only by age 31 may, in effect, also be an indication of the influence of these deep economic recession years (cf. the discussion in the introductory chapter). 
Figure 6.12: Main activity distributions (\%-share) by age 31 of young completers vs. young non-completers having completed an upper secondary degree by age 26 (late completers by 26) or only by age 31 (late completers by 31), or not having completed an upper secondary education by age 31 (adult non-completers), based on information on the 1993 youth cohort, by country
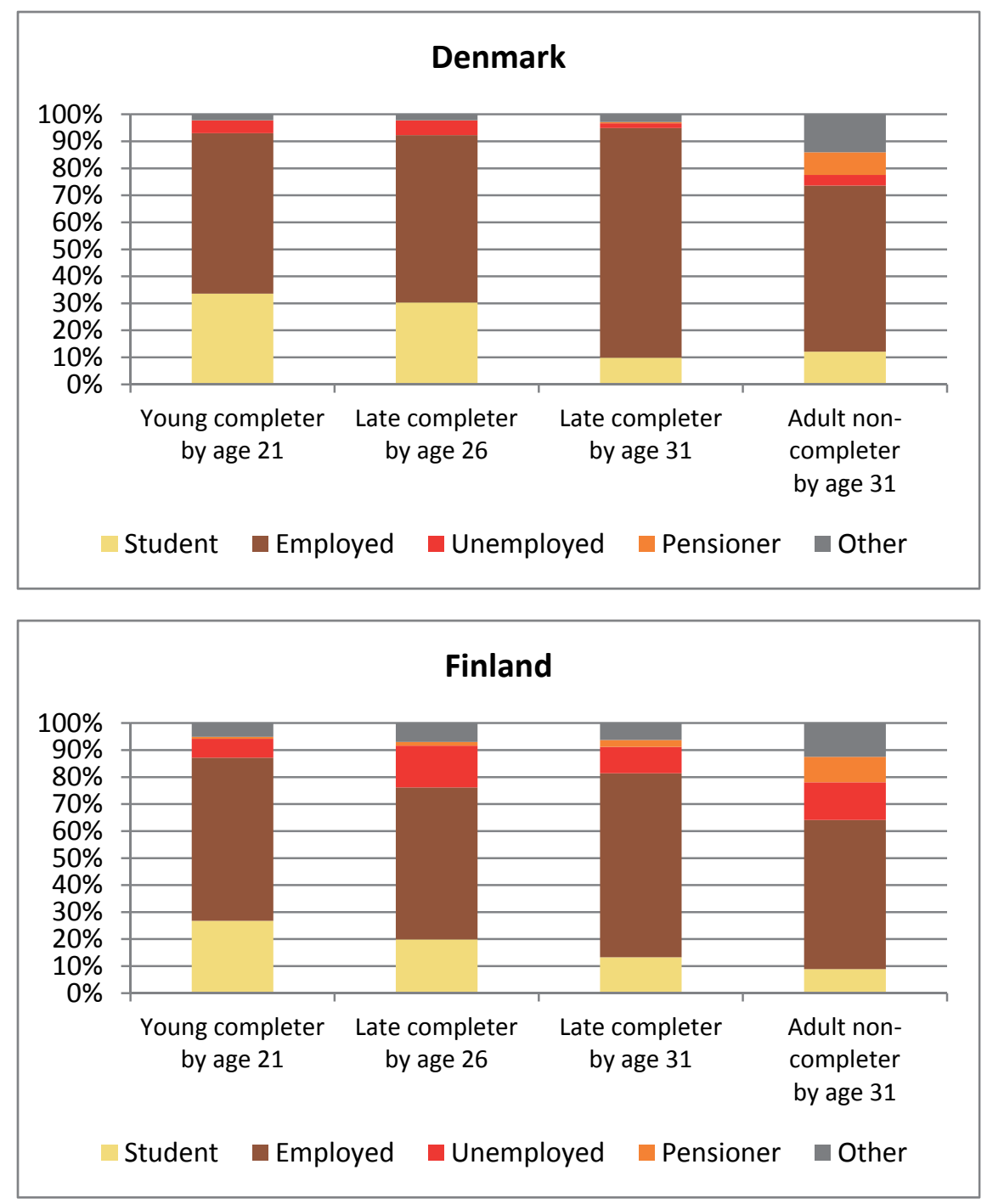

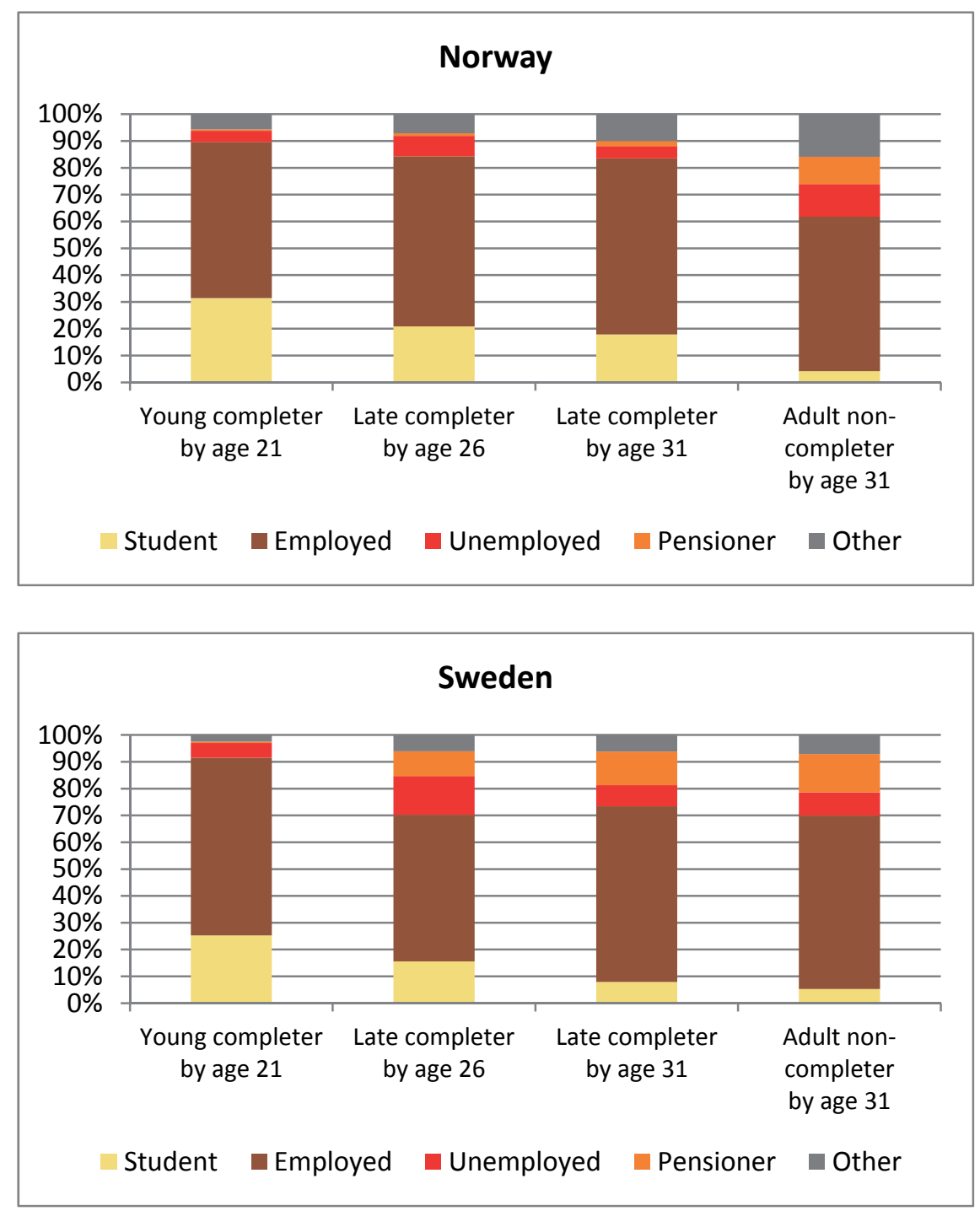

Notes: For definitions of the five main activity groups, see Chapter 2. Young completers = young people having completed an upper secondary education already by age 21 . Late completers by 26 = 21-year-old non-completers having achieved an upper secondary degree by age 26 (but not yet by age 21). Late completers by $31=21$-year-old non-completers having achieved an upper secondary degree by age 31 (but not yet by age 26). Adult non-completers = young people with only a basic education.

The second graph of Figure 6.12, showing the corresponding results for Finland, paints a slightly different picture, despite certain distinct features which Finland seems to share with Denmark. In particular, young completers are most successful with respect to labour market outcomes, followed by those having completed an upper secondary degree only by age 31. The lowest "activity" (education + employment) share is ob- 
tained for those having graduated by age 26 which, again, may well reflect the impact of the economic recession in the early 1990s. Another sign of the influence of these recession years is the higher shares of unemployed in the 1993 than in the 1998 cohort. Adult non-completers, on the other hand, are also in Finland distributed across main activities in much the same way as adult non-completers in the 1998 cohort: both reach up to an "activity" level of only some $65 \%$. The share of the unemployed is in both cohorts about $14 \%$, whereas about one-fifth has withdrawn outside the labour market.

The pattern emerging for Norway, as displayed in the third graph of Figure 6.12, indicates that the two groups of late completers of an upper secondary degree fare, by and large, equally well in terms of combined education and employment shares, but worse than young completers. There is, however, one major difference between the two groups of late completers: a relatively larger share of unemployed among those having graduated by age 26 and a relatively larger share having withdrawn from both education and the labour market among those having graduated only by age 31 . The weakest labour market situation is observed for adult non-completers. Indeed, their distribution across main activities is very similar to that of adult noncompleters in the 1998 cohort also in Norway.

The last graph of Figure 6.12 explores the situation at age 31 among young Swedes belonging to the 1993 cohort. Of the four groups of young people differing in their graduation history, only the young completers reach an "activity" share of almost 92\% when aged 31. This share is marginally lower than that of young completers in the 1998 cohort, mainly due to a slightly higher share of unemployed jobseekers among young completers in the 1993 cohort. The outcome for the other three groups of young people is notably weaker with an "activity" share of about $70 \%$, irrespective of late graduation or no graduation. Indeed, the main difference between the three groups of late completers and adult non-completers is not in their combined education and employment share but rather in the composition of their NEET activities: those having graduated by age 26 experience, when 31-year-old, more unemployment but less disability, with the situation being the opposite for those having graduated only by age 31 as well as for those having no post-compulsory degree still at age 31 . Perhaps most striking is the steady increase in the share of disability beneficiaries and also of those in other types of inactivity when going from young completers to late completers to adult non-completers. 


\subsubsection{Main findings}

This sub-chapter has focused on exploring whether there are distinct differences in terms of labour market outcomes between young completers, late completers and adult non-completers of an upper secondary degree. This comparison was made at two age points: among those aged 26 and among those aged 31 .

The results obtained for young people when aged 26 indicate the following. We can identify basically three patterns characterising the four Nordic countries under study. In Denmark, early and late completion of an upper secondary degree seems to make no difference in terms of labour market outcomes. Indeed, the distributions across main activities are almost identical for young completers and late completers, with most of them either studying or working at age 26. Adult noncompleters, on the other hand, are less likely to continue in education. They also face weaker employment possibilities and, consequently, a higher risk of ending up in NEET activities.

A second kind of pattern emerges for Finland and Norway. In both countries, young completers fare better than late completers, albeit the difference in labour market outcomes is more pronounced in Finland than in Norway. Conversely, the gap to adult non-completers is broader in Norway than in Finland. Indeed, the labour market situation of 26year-old non-completers is strikingly similar for these two countries, with almost $40 \%$ of them being in NEET activities.

Sweden, finally, is characterised by a third type of pattern. In particular, while young completers fare extremely well in terms of labour market outcomes, late completers lag far behind. A conspicuous finding is that there are only small, if any, differences in the distributions across main activities for late completers and adult non-completers. In both groups, about $30 \%$ are in NEET activities when aged 26 . Hence, adult non-completers do not seem to fare worse than late completers, in terms of labour market outcomes. Late completers seem to be the major loser group in this setting.

Taken together, these highly different young-completer/latecompleter/adult-non-completer patterns observed for Denmark, Finland, Norway and Sweden imply that late completers and adult noncompleters encounter highly different labour market situations in the four countries. Among late completers, Swedish late completers fare worst, whereas Danish late completers manage equally well as young completers in terms of labour market outcomes by age 26 . The ranking of countries changes slightly when comparing the position of adult noncompleters: Denmark shows up with the highest "activity" (education + 
employment) share also among adult non-completers, but now with Sweden coming second. Hence, while adult non-completers throughout fare much worse than young completers in terms of labour market outcomes in adulthood it is not obvious that late completion is always the second-best option. In particular, late completion can definitively be seen as a second-best option in Finland and Norway, but not necessarily in Denmark and Sweden, albeit for different reasons: in Denmark late completers fare equally well as young completers, whereas in Sweden late completers fare no better than adult non-completers.

When extending this exercise with five more years, up to age 31, the cross-country pattern becomes more dispersed. An additional circumstance affecting the pattern within as well as between countries relates to the economic recession in the early 1990s, as the results for the 31year-olds concern the 1993 cohort only. This impact shows up in, for instance, typically higher shares of unemployed among both early and late completers in the 1993 cohort, when compared to the 1998 cohort. This finding thereby also lends further support to the conclusion drawn earlier in this chapter in relation to the impact of economic shocks on young completers and non-completers; i.e., changing business cycles seem to typically impact on young people in much the same way irrespective of their upper secondary graduation background.

But despite the more mixed picture mediated by the results obtained for the 1993 cohort, there are also distinct country-specific features common to the results produced for the 1993 and 1998 cohorts, implying that much the same overall pattern can be identified for both cohorts. In particular, early and late completers tend to fare equally well in Denmark, while late completers fare worse than young completers in Finland and Norway, and much worse in Sweden. Moreover, the age of late completion does not seem to matter that much: the "activity" (education + employment) share is approximately the same and, hence, also the NEET share. However, there is considerable variation in the composition of late completers' NEET activities depending on the age of graduation.

The worst outcome is obtained for adult non-completers, except for Sweden in the sense that the labour market outcome of adult noncompleters is highly similar to that of late completers. Moreover, the distribution across main activities of adult non-completers is, in all four countries, approximately the same in the 1993 cohort as in the 1998 cohort. This indicates that the labour market situation of adult noncompleters looks more or less the same irrespective of cohort and changing economic environments. 


\section{Labour market outcomes in view of background}

In this chapter, we look into the background of our young people, with a view of trying to identify factors that seem to be especially strongly related to the labour market outcomes observed at three points in time: when these young persons turned 21, 26 and 31. Our statistical analysis is based on the use of so-called multinomial logit models which show the probability of belonging to one out of several mutually exclusive groups, given a particular set of background characteristics. In our case, these groups are made up of the five categories of main activities used in the previous chapters: full-time student, employed, unemployed, disability beneficiary or outside all of these activities ("other"). The background factors accounted for in these multinomial logit models divide basically into two main groups: one reflecting family background and the other early school-to-work-transition patterns, that is, trajectories followed straight after completion of compulsory education up to age 20. Additionally, we account for gender as well as cohort. Accounting also for cohort is relevant as we base our analysis on the pooled information available for all three youth cohorts under scrutiny, i.e., those young people who became 16 in 1993, 1998 and 2003, respectively.

In the following, we report our results by background factor, starting with a brief notion on the role of gender and cohort. Thereafter we turn to family background and, finally, to early school-to-work transitions. Each sub-chapter will also, when relevant, provide a short description of the background factor in question.

\subsection{Gender and labour market outcomes}

We start by comparing the average outcome for young men and women. More precisely, we compare the overall probability of young women to that of young men of being a full-time student, employed, unemployed, on disability benefits and in inactivity ("other") when aged 21, 26 and 31. This information is gathered into Table 7.1 for all four Nordic countries under study. It is worth noting that the reported differences in the proba- 
bility of young women, when compared to young men, of showing up in either one of the five main activity categories under scrutiny reflects the situation after taking into account differences in family background and early school-to-work-transition patterns, in addition to cohort.

\begin{tabular}{|c|c|c|c|c|c|}
\hline \multicolumn{2}{|c|}{ Labour market outcome } & \multirow{2}{*}{$\begin{array}{r}\text { Denmark } \\
0.079\end{array}$} & \multirow{2}{*}{$\begin{array}{l}\text { Finland } \\
0.131\end{array}$} & \multirow{2}{*}{$\begin{array}{r}\text { Norway } \\
0.106\end{array}$} & \multirow{2}{*}{$\begin{array}{r}\text { Sweden } \\
0.124\end{array}$} \\
\hline Full-time student & age 21 & & & & \\
\hline & age 26 & 0.048 & 0.013 & 0.021 & 0.065 \\
\hline & age 31 & 0.010 & 0.010 & 0.027 & 0.048 \\
\hline \multirow[t]{3}{*}{ Employed } & age 21 & -0.084 & -0.124 & -0.089 & -0.107 \\
\hline & age 26 & -0.053 & -0.053 & -0.012 & -0.055 \\
\hline & age 31 & -0.011 & -0.054 & -0.022 & -0.050 \\
\hline \multirow[t]{3}{*}{ Unemployed } & age 21 & 0 & -0.029 & -0.028 & -0.021 \\
\hline & age 26 & 0.006 & -0.020 & -0.014 & 0 \\
\hline & age 31 & 0.005 & -0.015 & 0 & 0.004 \\
\hline \multirow[t]{3}{*}{ Disability beneficiary } & age 21 & 0 & -0.001 & 0.003 & 0 \\
\hline & age 26 & 0 & -0.003 & 0.005 & 0.005 \\
\hline & age 31 & 0 & -0.003 & 0.006 & 0.013 \\
\hline \multirow[t]{3}{*}{ Other (inactive) } & age 21 & 0.008 & 0.023 & 0.007 & 0.004 \\
\hline & age 26 & 0 & 0.063 & 0 & -0.011 \\
\hline & age 31 & 0 & 0.062 & -0.009 & -0.014 \\
\hline
\end{tabular}

Notes: The reported average probabilities are obtained after also accounting for cohort, family background and early school-to-work-transition patterns. A negative sign implies a weaker probability of young women than of young men of showing up in the labour market situation in question. Low (high) absolute values indicate a small (large) difference in this respect to young men. All reported probabilities are significant at the $99 \%$ level or more. Statistically insignificant and only weakly significant probabilities are set at zero. The probabilities are calculated from pooled information on the three youth cohorts under scrutiny. For definitions of the five main activity categories, see Chapter 2.

Table 7.1 indicates the following. In all four countries, young women have a significantly higher probability of being in full-time education, when compared to their male peers. All four countries also share the feature of a notable narrowing in this gender gap when moving towards age 31 , with most of this change typically occurring before age 26, except in Denmark. The highest probability gap shows up for Finland with 21 -year-old women having a $13 \%$ higher probability than their male peers of being enrolled in full-time education. This probability is only slightly lower (12.4\%) for Sweden, but comparatively low (about 8\%) for Denmark. However, while this full-time-study probability in favour of young women remains comparatively high in Sweden up to age 31, it narrows remarkably in Finland and, in effect, down to the same low level (1\%) as in Denmark.

Conversely, young men have throughout, at all three age points, a clearly higher probability of being in working life. However, again we see a declining trend with age in the probability gap between the two gen- 
ders: a smoothly narrowing gap in Denmark, but a more step-wise decline in the other three countries in the sense that most of also this narrowing seems to take place before the age of 26. In view of the high probability gap obtained for full-time studying 21-year-old Finns, it is hardly surprising that Finland comes out with the highest gender gap also in the probability of 21 -year-olds being in employment $(12.4 \%$ in favour of men), again closely followed by Sweden (close to $11 \%$ in favour of men). In Denmark and Norway, the corresponding gap is just below $9 \%$. However, this higher probability of young men being in working life has, in all countries, shrunk to about $5 \%$ already by age 26 , in Norway to only about $1 \%$.

The difference between young men and young women in the average probability of showing up in (registered) unemployment is small or nonexistent at all three age points investigated. Moreover, this holds true for all four countries. Even the largest differences fall below 3\% (to the favour of women) and are observed only at the age of 21 and only for three out of the four countries under study.

Also the differences in average gender probabilities of being in disability arrangements are more or less negligible in all four countries. In Denmark, there exist principally no differences between young men's and young women's probabilities of being on disability benefits. This pattern is repeated at all three age points. In Finland, young women have a marginally lower probability in this respect, and the difference to young men remains approximately unchanged up to age 31. In Norway, the situation is reversed. Additionally, young women tend to face a weakly increasing probability, when compared to young men, of ending up as disability beneficiaries. This pattern and trend is even more pronounced for young women in Sweden.

When it comes to the probability of young men and young women moving into other types of inactivity, the differences in probabilities are, again, small or negligible. The only exception is Finland where young women have a comparatively high probability of withdrawing from both education and the labour force, when compared to their male peers. Moreover, this difference in inactivity probabilities is much higher at age 26, and also at age 31, than at age 21. Also the results for Sweden are worth commenting on in the sense that while young women tend to face an increasingly higher probability of going into disability arrangements when approaching age 31, young men tend instead to increasingly withdraw into other types of inactivity. 


\subsubsection{Main findings}

The largest differences in young men's and young women's average probabilities of showing up in alternative labour market situations when aged 21, 26 and 31, respectively, relate to full-time education and employment. In particular, young women face a much higher probability of being enrolled in full-time education, whereas young men are much more likely to enter working life. While these differences in education and employment probabilities prevail across all three age points investigated, they diminish substantially with age especially up to age 26 , with more minor changes occurring beyond age 26 , up to age 31 . This overall pattern is discernible in all four countries, implying that there is a notable convergence over time not only across genders but also across countries.

When it comes to NEET activities, the differences in gender probabilities are notably smaller. Also this pattern emerges for all four countries. For Denmark, the differences in gender probabilities are consistently minor or non-existent, suggesting that young men and young women in Denmark are on average equally likely to become unemployed, to go on disability benefits and to withdraw into other types of inactivity. This holds, by and large, true also for Norway, except when it comes to unemployment. In this respect, young men face a clearly higher risk than young women, although the difference is small and vanishes with age. Finland stands out with a persistently, albeit only marginally, higher probability of young men becoming unemployed or of moving into disability arrangements, whereas young women have a strikingly higher probability of withdrawing into other types of inactivity, especially beyond age 21. A reversed pattern appears for Sweden with young women being on average more likely to go on disability benefits and young men into other (unknown) types of inactivity. While these country-specific patterns were pointed out already in the previous chapter, they thus seem to be retained also after control for differences in cohort, family background and early school and labour market experiences. 


\subsection{Cohort and labour market outcomes}

Next, we look briefly at differences in the average probability for the three youth cohorts under study of being in these same five alternative labour market situations at age 21 and 26 after also controlling for gender, family background and early school-to-work-transition patterns. At age 21, we can make comparisons across all three cohorts as we have observations for all of them five years after completion of compulsory school. At age 26, this comparison boils down to two cohorts (the 1993 and 1998 cohorts of 16-year-olds) for which we have observations up to ten years after completion of compulsory education. For age 31, no corresponding comparisons can be made as the information at this particular age concerns the oldest cohort only, i.e. the 1993 cohort of 16-year-olds.

Again, the differences in average probabilities reported here (in Tables 7.2 and 7.3) are those obtained after accounting for the full set of background factors. However, in contrast to the situation for gender, we see considerable variation in the relation between cohort and labour market outcomes depending on whether or not we leave out information on either family background or early school-to-work transitions. This observation points to a changing role of these two background factors across cohorts. This lends further support to including both sets of background factors when analysing data containing information on more than one cohort and when, furthermore, trying to explore whether there are distinct differences between cohorts in labour market outcome probabilities in adulthood.

\subsubsection{Differences in average labour market outcome probabilities at age 21}

From Table 7.2 it is evident that the average probability of being in either one of the five alternative labour market situations when aged 21 reveals remarkable variation across cohorts within countries, as well as within cohorts across countries. The probability of being a full-time student at age 21 has increased across cohorts in Denmark, stayed unchanged in Norway, and followed a concave-type trend in Finland and Sweden. The Danish result is well in line with previous observations of more young Danes continuing in education and increasingly also delaying their completion of an upper secondary degree (cf. Chapter 2). The Finnish and Swedish results, in turn, are likely to reflect the growing tendency across cohorts of young people delaying their start in higher education. 
Table 7.2: 1998 and 2003 cohort probabilities in terms of labour market outcomes at age 21, by country; differences in average probabilities when compared to the 1993 cohort

\begin{tabular}{|c|c|c|c|c|}
\hline Labour market outcome & Denmark & Finland & Norway & Sweden \\
\hline \multicolumn{5}{|l|}{ Full-time student } \\
\hline cohort 1998 & 0.030 & 0.019 & 0 & 0.020 \\
\hline cohort 2003 & 0.066 & -0.019 & 0 & -0.037 \\
\hline \multicolumn{5}{|l|}{ Employed } \\
\hline cohort 1998 & -0.040 & 0 & 0 & -0.012 \\
\hline cohort 2003 & -0.053 & 0.059 & 0.011 & 0.041 \\
\hline \multicolumn{5}{|l|}{ Unemployed } \\
\hline cohort 1998 & 0.009 & -0.019 & 0.034 & -0.028 \\
\hline cohort 2003 & -0.033 & -0.036 & 0.013 & -0.051 \\
\hline \multicolumn{5}{|l|}{ Disability beneficiary } \\
\hline cohort 1998 & -0.002 & -0.002 & 0 & 0.003 \\
\hline cohort 2003 & -0.002 & 0 & 0 & 0.012 \\
\hline \multicolumn{5}{|l|}{ Other (inactive) } \\
\hline cohort 1998 & 0 & 0 & -0.038 & 0.016 \\
\hline cohort 2003 & 0.022 & -0.005 & -0.021 & 0.034 \\
\hline
\end{tabular}

Notes: The reported average probabilities are obtained after also accounting for gender, family background and early school-to-work-transition patterns. A negative sign implies a weaker probability of the cohort's young people of showing up in the labour market situation in question compared to young people from the 1993 cohort. Low (high) absolute values indicate a small (large) difference in this respect to the 1993 cohort. All reported probabilities are significant at the $99 \%$ level or more. Insignificant and only weakly significant probabilities are set to zero. The probabilities are calculated from pooled information on the three youth cohorts under study. For definitions of the five main activity groups, see Chapter 2.

The average probability of 21-year-olds of being in working life is more or less the same for cohorts 1993 and 1998, but significantly higher for the 2003 cohort, notably in Finland and Sweden. For Denmark, on the other hand, we see a steady decline across cohorts in the probability of 21-yearolds being in employment. These findings are the logical counterpart to the country-specific trends in studying probabilities discussed above.

In all countries, except Norway, the average probability of being registered as an unemployed jobseeker when aged 21 is clearly lower in the youngest (2003) cohort than in the oldest (1993) cohort. For Finland and Sweden, this holds true also for the 1998 cohort. These different findings for the four Nordic countries, despite the fact that all of them experienced a deep economic recession in the early 1990s, may seem rather surprising. However, the interpretation of these differences in average unemployment probabilities across the three cohorts is not necessarily straightforward, for several reasons. First, unemployment refers to registered unemployment and, as pointed out earlier, young people not eligible for unemployment benefits might choose not to sign on. This behaviour may well have become more common with tightened conditions, especially for young people, for receiving unemployment benefits. Second, youth unemployment policies have increased both in 
volume and in intensity over the past decade or so, but in quite different ways in the four Nordic countries.

Also when it comes to disability benefits and other types of inactivity, the cross-country picture appears quite mixed. In all countries, except Sweden, we see minor cross-cohort differences in young people's probability of being on disability benefits already at age 21 . For Sweden, we observe instead a weakly increasing probability across cohorts of 21year-olds showing up in disability arrangements. A similar trend is, in fact, observable for Sweden also when it comes to other types of inactivity. Also for Denmark, the average probability of withdrawal from both education and the labour force when aged 21 is slightly higher in the 2003 cohort than in the older cohorts, whereas the opposite trend is discernible for Finland and Norway.

\subsubsection{Differences in average labour market outcome probabilities at age 26}

In the next table, we expand this picture for the 21-year-olds with corresponding results five years later, at age 26 . As noted above, the comparison across cohorts at this age is, due to data limitations, restricted to only two cohorts (cohorts 1993 and 1998). In order to facilitate our comparison of probabilities at age 21 and age 26, Table 7.3 reproduces the probabilities of the 1998 cohort at age 21, as reported in Table 7.2. This allows us to easily compare the situation of the 1993 cohort to that of the 1998 cohort at two specific age points.

\begin{tabular}{|c|c|c|c|c|}
\hline Labour market outcome & Denmark & Finland & Norway & Sweden \\
\hline \multicolumn{5}{|l|}{ Full-time student } \\
\hline cohort 1998, age 21 & 0.030 & 0.019 & 0 & 0.020 \\
\hline cohort 1998, age 26 & 0.028 & 0 & -0.037 & -0.015 \\
\hline \multicolumn{5}{|l|}{ Employed } \\
\hline cohort 1998, age 21 & -0.040 & 0 & 0 & -0.012 \\
\hline cohort 1998, age 26 & 0.008 & 0.031 & 0.015 & 0.021 \\
\hline \multicolumn{5}{|l|}{ Unemployed } \\
\hline cohort 1998, age 21 & 0.009 & -0.019 & 0.034 & -0.028 \\
\hline cohort 1998 , age 26 & -0.051 & -0.020 & -0.013 & -0.028 \\
\hline \multicolumn{5}{|l|}{ Disability beneficiary } \\
\hline cohort 1998, age 21 & -0.002 & -0.002 & 0 & 0.003 \\
\hline cohort 1998, age 26 & 0 & 0 & 0.003 & 0.006 \\
\hline \multicolumn{5}{|l|}{ Other (inactive) } \\
\hline cohort 1998, age 21 & 0 & 0 & -0.038 & 0.016 \\
\hline cohort 1998, age 26 & 0.015 & -0.009 & 0.031 & 0.016 \\
\hline
\end{tabular}

Notes: See Table 7.2 above. 
The overall impression mediated by Table 7.3 is that there is no clearcut cross-country pattern discernible in the labour market outcome probabilities of the two cohorts when moving from age 21 to age 26. In Norway and Sweden, 26-year-olds from the 1998 cohort are less likely to be in education than were 26-year-olds from the 1993 cohort. This could be argued to be a consequence of the deep economic recession in the early 1990s spurring young people to stay longer in education due to sluggish employment opportunities. However, this same pattern does not emerge for Finland, where the young people from the two cohorts are instead equally likely to be full-time students still at age 26 , nor for Denmark, where the higher probability of the 1998 cohort of being engaged in education is of the same magnitude at age 26 as five years earlier, at age 21.

The different economic situations faced by young people from the two cohorts upon labour market entrance seem to play a more distinct role when it comes to employment probabilities. As shown in Table 7.3, the average employment probability at age 26 is in all four countries clearly higher for the 1998 cohort than for the 1993 cohort, more so in Finland and Sweden than in Denmark and Norway. Concomitantly, the 1998 cohort's unemployment risk at this particular age is in all four countries lower than for the 1993 cohort, especially in Denmark. In Finland and Sweden, the difference in unemployment probabilities between the two cohorts is, in effect, the same at age 26 as it was at age 21 .

Concerning disability benefits, the differences in probabilities between the two cohorts are minor. Yet, the trend is increasing rather than decreasing. In particular, in both Denmark and Finland, the 1998 cohort started out, at age 21, from a lower probability level than cohort 1993, but this gap was closed by age 26 . In the other two countries, the probability of going on disability benefits already at age 21 was the same (Norway) or marginally higher (Sweden), when compared to the 1993 cohort. By age 26, this risk had increased more rapidly in the 1998 cohort, albeit it was still only marginally higher than for the 1993 cohort.

Finally, with respect to other types of inactivity cohort 1998 comes out with a notably higher risk of being outside both education and the labour force at age 26. In Norway, there is a remarkable change between age 21 (an almost 4\% higher risk for cohort 1993) and age 26 (a 3\% lower risk for cohort 1993). In Sweden, this higher risk for cohort 1998 was observable already at age 21. Only in Finland is this inactivity risk lower at age 26 in the 1998 cohort than in the 1993 cohort, but only marginally so. 


\subsubsection{Main findings}

The labour market outcome probabilities of young people belonging to different cohorts reveal considerable variation both within and across countries, showing no clear-cut overall patterns whatsoever. A first observation is that the differences in probabilities between cohorts are throughout quite small in size, implying that the probability of young people ending up in different labour market situations has not changed that much across cohorts after also controlling for cohort-specific differences in family background and early school-to-work transitions. A second observation is that there is typically no clear pattern of changing (or unchanging) probabilities across cohorts when going from age 21 to age 26 .

A comparison across the three cohorts of the labour market outcome probabilities of young people when aged 21 implies that the probability of being a full-time student was lower for the 2003 cohort than for the 1998 and 1993 cohorts in both Finland and Sweden, obviously due to break years having become increasingly common before continuing in higher education. This contention is supported by working being much more likely among 21-year-olds in the 2003 cohort than in the two older cohorts. For Denmark, the findings are rather the opposite with increasing probabilities across cohorts of being engaged in education and decreasing probabilities of being in employment when aged 21. For Norway, on the other hand, there are minor, if any, differences in cohort probabilities when it comes to studying and working.

In relation to various NEET activities among 21-year-olds, the mix of cross-cohort patterns within and between countries is much larger than for education and employment probabilities, which is only to be expected in view of the results presented in previous chapters. The mostly very small or non-existent differences in NEET probabilities across cohorts confirm, in turn, the impression of strikingly stable prevalence of NEET activities among young people. In particular, the risk of being outside both education and the labour market - in disability arrangements or other types of inactivity - when aged 21 reveals an increasing rather than decreasing trend across cohorts. This holds true especially for Sweden, but also for Denmark. Only with respect to unemployment do we see a clearly lower risk among 21-year-olds from the 2003 cohort, though again with Norway being an exception to this pattern.

A comparison of labour market outcome probabilities at age 26 now based on only two cohorts - induces basically three main observations. First, the probability of being employed at age 26 is in all four countries notably higher in the 1998 cohort than in the 1993 cohort. Second, the risk of being unemployed is clearly lower. Taken together, 
these two observations are likely to mirror the impact of the deep economic recession in the early 1990s on young labour market entrants. Third, the differences between the two cohorts in the risk of being outside both education and the labour force at age 26 are mostly small or minor and, in effect, very similar to those observed among the 21-yearolds. This points to rather stable inactivity patterns across cohorts also in adulthood. Indeed, also at age 26 the risk of ending up in inactivity has been increasing rather than decreasing across cohort, a tendency which now shows up for all countries except Finland.

\subsection{Family background and labour market outcomes}

Intergenerational transmission from parents to children has for long been an important academic as well as political issue. Special attention has thereby been paid to the parents' educational and income levels. Indeed, as shown by e.g. Björklund et al. (2010) and Black and Devereux (2011) in their comprehensive reviews, there is a huge body of literature providing support for the contention that school success and, ultimately, labour market outcomes are closely related to family background. It is therefore of interest to include also in this context measures approximating the family background situation of the young people belonging to the three cohorts investigated.

We measure family background by use of a small set of traditional family background measures common to all four Nordic countries under study. More precisely, we ask whether there is a clear-cut relation between the family situation as measured by education and income and the child's probability of being a full-time student, employed, unemployed, disability beneficiary or inactive ("other") at age 21, 26 and 31, respectively. The parents' formal educational level is measured by means of three categories: basic, secondary and higher education. Also the (gross) income level of parents is split into three categories: low, middle and high wage-income. While the information on educational level is given separately for the mother and the father, the wage-income refers to the household-level income, i.e. the sum of the parents' wageincome. The parents' education and income concern the year when the child turned 16, except for Finland as the Finnish data contains parental education information for the year 2010 only.

While this family background information (mother's and father's education, parental wage-income level) is added jointly to our statistical model, we split the presentation of results, starting with the relation 
between the mother's educational level and the child's labour market outcomes at later ages. Apart from family background information, the model also accounts for gender and cohort as well as early school-towork transitions.

\subsubsection{The role of the mother's educational level}

Table 7.4 reports the extent to which the educational level of the mother is linked to the child's later labour market outcomes. In particular, it gives the differences in probabilities for the child being in either one of the five alternative labour market situations at age 21, 26 and 31, respectively, depending on the educational level completed by the mother when the child turned 16. These differences in probabilities are reported for mothers with an upper secondary or a higher education. In other words, mothers with no post-compulsory degree act as the reference group.

\begin{tabular}{|c|c|c|c|c|c|c|c|c|}
\hline \multirow[b]{3}{*}{ Labour market outcome } & \multicolumn{8}{|c|}{ Mother's highest educational level: secondary- or tertiary-level degree } \\
\hline & \multicolumn{2}{|c|}{ Denmark } & \multicolumn{2}{|c|}{ Finland } & \multicolumn{2}{|c|}{ Norway } & \multicolumn{2}{|c|}{ Sweden } \\
\hline & sec. ed. & tert. ed. & sec. ed. & tert. ed. & sec. ed. & tert. ed. & sec. ed. & tert. ed. \\
\hline \multicolumn{9}{|l|}{ Full-time student } \\
\hline at age 21 & 0.039 & 0.065 & 0.047 & 0.156 & 0.064 & 0.152 & 0.045 & 0.175 \\
\hline at age 26 & 0.035 & 0.148 & 0.023 & 0.098 & 0.040 & 0.130 & 0.028 & 0.118 \\
\hline at age 31 & 0 & 0.048 & 0 & 0.047 & 0 & 0.031 & 0 & 0.029 \\
\hline \multicolumn{9}{|l|}{ Employed } \\
\hline at age 21 & -0.027 & -0.063 & -0.035 & -0.114 & -0.041 & -0.122 & -0.019 & -0.136 \\
\hline at age 26 & -0.023 & -0.142 & -0.011 & -0.065 & -0.026 & -0.114 & -0.012 & -0.101 \\
\hline at age 31 & 0 & -0.049 & 0 & -0.038 & 0 & -0.026 & 0.008 & -0.014 \\
\hline \multicolumn{9}{|l|}{ Unemployed } \\
\hline at age 21 & -0.008 & -0.011 & -0.007 & -0.033 & -0.011 & -0.021 & -0.018 & -0.038 \\
\hline at age 26 & -0.005 & -0.007 & -0.006 & -0.020 & -0.011 & -0.017 & -0.011 & -0.019 \\
\hline at age 31 & 0 & 0 & 0 & -0.010 & -0.007 & -0.012 & -0.006 & -0.011 \\
\hline \multicolumn{9}{|l|}{ Disability beneficiary } \\
\hline at age 21 & 0 & 0 & 0 & 0 & 0 & 0 & -0.004 & -0.007 \\
\hline at age 26 & 0 & 0 & 0 & 0 & 0 & 0 & -0.003 & -0.006 \\
\hline at age 31 & 0 & 0 & 0 & 0 & 0 & 0 & 0 & -0.009 \\
\hline \multicolumn{9}{|l|}{ Other (inactive) } \\
\hline at age 21 & -0.005 & 0.009 & -0.005 & -0.009 & -0.011 & -0.010 & -0.004 & 0.006 \\
\hline at age 26 & -0.007 & 0 & -0.005 & -0.011 & 0 & 0 & 0 & 0.009 \\
\hline at age 31 & 0 & 0 & 0 & 0 & 0 & 0 & 0 & 0 \\
\hline
\end{tabular}

Notes: The three educational-level categories correspond to ISCED 1-2, 3-4 and 5-6, respectively, with ISCED 1-2 (no post-compulsory educational degree) being used as the category of reference. The Norwegian results also include a category for missing educational information which is not reported here, though. For other notes, see Table 7.2 above. 
Table 7.4 presents results concerning the child's probability of being engaged in education later in life that are well in line with the empirical evidence reported in the international literature. In particular, there is a strong link between the child's probability of continuing in education and the mother's educational level. This relation strengthens at all three age points with the level of the mother's completed degree: compared to mothers with no post-compulsory degree, this link is notably stronger for mothers with an upper secondary degree and strongest for high-educated mothers. However, the table also reveals that the role of the mother's educational background weakens with the child growing older: for mothers with an upper secondary degree, the link is broken by age 31 while it has turned quite weak for mothers with a tertiary-level degree. This overall pattern is repeated for all four countries, but with the strength of the relation varying a lot between the countries. The cross-country situation is most similar at age 31 , which again points to considerable convergence with age in the educational behaviour of Nordic youth.

Not surprisingly, the counterpart to this educational pattern is employment probabilities of the child that are negatively related to the mother's educational background. In other words, for the probability of the child being in employment - instead of continuing in education - we observe a reversed pattern: the child's probability of having entered working life already by age 21 declines notably with the educational level of the mother, implying that it is highest for children with a mother lacking a postcompulsory degree. Again, this same pattern is repeated for each of the three age points investigated, but with the link to the mother's education weakening also in the case of employment probabilities when the child grows older. And again, the same pattern emerges for all four countries.

The child's risk of ending up in NEET activities - unemployment or inactivity, including disability benefits - is, on average, much more weakly related to the mother's educational background than is the child's probability of studying or working. There is, in all four countries, a negative but quite weak link between the child's risk of experiencing unemployment and the mother's educational background, with this relationship weakening further when the child grows older. When it comes to the child's probability of becoming a disability beneficiary, the mother's educational background plays no significant role, except in Sweden where the link seems to exist but in an extremely weak mode. Also in relation to the child's risk of ending up in other types of inactivity, the link to the mother's educational background is minor or non-existent. Again, the same overall pattern shows up in all four countries. 


\subsubsection{The role of the father's educational level}

Next, we turn to the corresponding results for the father's highest completed education. The relevant probabilities are presented in Table 7.5. By and large, the picture looks much the same, suggesting that the child's labour market outcomes later in life are related to the mother's and the father's educational background in a highly similar way. Moreover, this holds true for both the sign and the strength of these relations. For all four countries, we observe studying probabilities of the child that are positively related and working probabilities that are negatively related to the father's educational background. This link strengthens markedly with the educational degree completed by the father. Moreover, while this pattern is repeated when the child turns 21,26 and 31 , also the relation to the father's educational background tends to weaken considerably with the child growing older. Likewise, the risk of the child of ending up in NEET activities is only weakly, if at all, related to the father's education.

Table 7.5: Probabilities in terms of labour market outcomes at age 21, 26 and 31, respectively, of children with a higher educated father, by country; differences in probabilities when compared to children with a father lacking a post-compulsory education

\begin{tabular}{|c|c|c|c|c|c|c|c|c|}
\hline \multirow[b]{3}{*}{ Labour market outcome } & \multicolumn{8}{|c|}{ Father's highest educational level: secondary- or tertiary-level degree } \\
\hline & \multicolumn{2}{|c|}{ Denmark } & \multicolumn{2}{|c|}{ Finland } & \multicolumn{2}{|c|}{ Norway } & \multicolumn{2}{|c|}{ Sweden } \\
\hline & sec. ed. & tert. ed. & sec. ed. & tert. ed. & sec. ed. & tert. ed. & sec. ed. & tert. ed \\
\hline \multicolumn{9}{|l|}{ Full-time student } \\
\hline at age 21 & 0.032 & 0.098 & 0.035 & 0.156 & 0.059 & 0.163 & 0.033 & 0.180 \\
\hline at age 26 & 0.018 & 0.136 & 0.019 & 0.103 & 0.037 & 0.129 & 0.022 & 0.100 \\
\hline at age 31 & 0 & 0.031 & 0 & 0.037 & 0.016 & 0.034 & 0 & 0.027 \\
\hline \multicolumn{9}{|l|}{ Employed } \\
\hline at age 21 & -0.025 & -0.101 & -0.028 & -0.116 & -0.044 & -0.141 & -0.023 & -0.162 \\
\hline at age 26 & 0 & -0.140 & -0.016 & -0.087 & -0.024 & -0.122 & -0.011 & -0.098 \\
\hline at age 31 & 0 & -0.034 & 0 & -0.027 & 0 & -0.035 & 0.009 & -0.022 \\
\hline \multicolumn{9}{|l|}{ Unemployed } \\
\hline at age 21 & -0.005 & -0.011 & 0 & -0.034 & -0.009 & -0.014 & -0.005 & -0.022 \\
\hline at age 26 & -0.007 & 0 & 0 & -0.015 & -0.006 & -0.010 & -0.006 & -0.009 \\
\hline at age 31 & 0 & 0 & 0 & -0.010 & 0 & 0 & -0.005 & 0 \\
\hline \multicolumn{9}{|l|}{ Disability beneficiary } \\
\hline at age 21 & 0 & 0 & 0 & 0 & 0 & 0 & -0.002 & -0.003 \\
\hline at age 26 & 0 & 0 & 0 & 0 & 0 & 0 & -0.003 & 0 \\
\hline at age 31 & 0 & 0 & 0 & 0 & 0 & 0 & -0.004 & 0 \\
\hline \multicolumn{9}{|l|}{ Other (inactive) } \\
\hline at age 21 & 0 & 0.012 & -0.005 & -0.008 & 0 & -0.008 & -0.004 & 0.007 \\
\hline at age 26 & 0 & 0 & 0 & 0 & 0 & 0 & 0 & 0.009 \\
\hline at age 31 & 0 & 0 & 0 & 0 & 0 & 0 & 0 & 0 \\
\hline
\end{tabular}

Notes: See Table 7.4 above. 


\subsubsection{The role of the parents' wage-income level}

The third and final family-related background factor concerns the parents' total wage income. The probabilities of young people of showing up in alternative labour market activities at age 21, 26 and 31, respectively, given observed differences in parents' wage-income levels are displayed in Table 7.6. Other background factors accounted for in this context are, in line with the overall setting used, mother's and father's educational levels, as well as the young person's gender, cohort and early school-towork-transition experiences.

\begin{tabular}{|c|c|c|c|c|c|c|c|c|}
\hline \multirow[b]{3}{*}{ Labour market outcome } & \multicolumn{8}{|c|}{ Parents' wage-income level: 2 nd tertile or 3 rd tertile } \\
\hline & \multicolumn{2}{|c|}{ Denmark } & \multicolumn{2}{|c|}{ Finland } & \multicolumn{2}{|c|}{ Norway } & \multicolumn{2}{|c|}{ Sweden } \\
\hline & $\begin{array}{r}\text { 2nd } \\
\text { tertile }\end{array}$ & $\begin{array}{r}\text { 3rd } \\
\text { tertile }\end{array}$ & $\begin{array}{l}\text { 2nd } \\
\text { tertile }\end{array}$ & $\begin{array}{r}\text { 3rd } \\
\text { tertile }\end{array}$ & $\begin{array}{l}\text { 2nd } \\
\text { tertile }\end{array}$ & $\begin{array}{r}\text { 3rd } \\
\text { tertile }\end{array}$ & $\begin{array}{r}\text { 2nd } \\
\text { tertile }\end{array}$ & $\begin{array}{r}\text { 3rd } \\
\text { tertile }\end{array}$ \\
\hline \multicolumn{9}{|l|}{ Full-time student } \\
\hline at age 21 & 0.016 & 0.067 & 0.027 & 0.074 & 0.021 & 0.058 & -0.011 & 0.035 \\
\hline at age 26 & 0 & 0.038 & 0 & 0.046 & 0 & 0.027 & -0.011 & 0.012 \\
\hline at age 31 & 0 & 0 & 0 & 0.014 & 0 & 0 & -0.010 & -0.013 \\
\hline \multicolumn{9}{|l|}{ Employed } \\
\hline at age 21 & 0.009 & -0.030 & 0 & 0 & 0.011 & -0.013 & 0.074 & 0.060 \\
\hline at age 26 & 0.024 & 0 & 0.030 & 0 & 0.026 & 0 & 0.067 & 0.063 \\
\hline at age 31 & 0.023 & 0.029 & 0.026 & 0.034 & 0.033 & 0.023 & 0.063 & 0.080 \\
\hline \multicolumn{9}{|l|}{ Unemployed } \\
\hline at age 21 & -0.009 & -0.021 & -0.021 & -0.047 & -0.012 & -0.023 & -0.038 & -0.066 \\
\hline at age 26 & -0.013 & -0.019 & -0.017 & -0.035 & -0.012 & -0.013 & -0.027 & -0.039 \\
\hline at age 31 & 0 & -0.009 & -0.016 & -0.030 & -0.008 & 0 & -0.019 & -0.026 \\
\hline \multicolumn{9}{|l|}{ Disability beneficiary } \\
\hline at age 21 & 0 & 0 & 0 & 0 & -0.004 & -0.005 & -0.006 & -0.012 \\
\hline at age 26 & 0 & 0 & -0.002 & -0.004 & -0.005 & -0.006 & -0.009 & -0.016 \\
\hline at age 31 & 0 & -0.007 & -0.004 & -0.006 & 0 & 0 & -0.016 & -0.023 \\
\hline \multicolumn{9}{|l|}{ Other (inactive) } \\
\hline at age 21 & -0.016 & -0.016 & -0.015 & -0.018 & -0.016 & -0.017 & -0.018 & -0.018 \\
\hline at age 26 & -0.012 & -0.012 & -0.015 & -0.019 & -0.013 & -0.009 & -0.020 & -0.021 \\
\hline at age 31 & -0.009 & 0 & -0.007 & -0.011 & -0.019 & 0 & -0.018 & 0.018 \\
\hline
\end{tabular}

Notes: The three wage-income categories of parents refer, respectively, to the lowest, middle and highest one-third of the wage-income scale. For other notes, see Table 7.2 above.

A first observation based on Table 7.6 is that parents' wage-income level is to a varying degree related to the child's probability of later in life being either studying, working, in unemployment, on disability benefits or in other types of inactivity, even after accounting for the parents' educational background. This means that the wage-income level of parents plays an independent role for the child's later labour market outcomes; it does not merely act as a proxy for parents' educational background. 
And vice versa, parents' educational background is not necessarily a sufficient proxy for their labour market income status. Hence, these two family background factors are correlated, but no perfect correlates. Moreover, these two family background indicators are, in this context, occasionally oppositely related to the child's later labour market outcomes, as discussed below.

Children with higher wage-income parents are, on average, more likely to be enrolled in full-time education when aged 21, when compared to children with low wage-income parents. Moreover, this positive relation strengthens with the wage-income level of parents and, hence, is strongest for parents belonging to the highest one-third of the wageincome scale. However, this relation between the child's later probability of being engaged in full-time studies and the parents' wage-income level weakens rapidly when the child grows older: for parents located in the middle part of the wage-income scale it has disappeared before the child turns 26 and for parents in the highest one-third of the wage-income scale it has turned very small or insignificant by the time the child turns 31. Only for Sweden does this pattern look different. In particular, children of parents located in the middle part of the wage-income scale show up with the lowest probability of being engaged in full-time studies at all three age points investigated. For high-income parents, in turn, the link to the child's probability of studying on a full-time basis later in life has, by age 31, turned from positive to negative. Hence, the probability of studying when aged 31 tends to be highest for children with lowincome parents. On the whole, though, this diverging pattern observed for Sweden builds on very small differences in the child's studying probability across the three parental wage-income levels.

The probability of the child of being in employment when aged 21, 26 and 31 is only weakly related to the parents' wage-income level. Moreover, this relation is typically positive and increasing with the child's age (i.e. stronger at age 31 than at age 21), which is opposite to the relation observed for the mother's and the father's educational background. These different types of relations are logical, though: while the parents' educational background is likely to mirror the child's probability of substituting work with studies, their wage-income level rather tells about the child's later employment prospects. Also with respect to the child's later employment probability we observe the same overall pattern for Denmark, Finland and Norway. Again, the pattern is different for Sweden: a relatively strong, albeit still positive, relation between the child's probability of being employed later in life and the parents' wage-income level. However, this difference appears mainly with respect to low- 
income parents, whereas the difference in the children's later employment prospects is minor or negligible when comparing middle-income parents to high-income parents.

Children with low-income parents tend to have the highest risk of experiencing unemployment later in life. This risk declines with the parents' wage-income level and, hence, is lowest for children with highincome parents. However, the link between the child's likelihood of experiencing unemployment in adulthood and the parents' wage-income level turns increasingly weaker when the child grows older. It is worth noting, though, that this negative relation remains quite strong in both Finland and Sweden: children with middle-income parents have still by age 31 an almost two per cent and children with high-income parents an almost three per cent lower probability of being unemployed, when compared to children with low-income parents.

The child's probability of moving later in life into disability arrangements is, at most, weakly negatively related to the parents' wage-income level. Moreover, when such a link exists, it typically strengthens slightly when the child grows older. In other words, the probability of the child of being on disability benefits is, on average, more strongly linked to the parents' wage-income when the child is 31 than when it is 21 . This pattern is most pronounced for Sweden with the difference in children's probability of being on disability benefits having by age 31 increased to $1.6 \%$ to the favour of those with middle-income parents and to $2.3 \%$ to the favour of those with high-income parents, when compared to the situation at age 31 of children with low-income parents.

For other types of inactivity, the overall pattern is again quite different, albeit still highly similar across the four Nordic countries under study. In particular, we observe a weak but significantly negative relation between the child's risk of ending up outside both education and the labour market and the parents' wage-income level. Moreover, this difference in risks occurs between children with low-income and children with higher-income parents, whereas there is basically no difference in this respect between children with middle-income and high-income parents. Furthermore, this clearly higher risk of children with lowincome parents of moving into inactivity remains practically unchanged up to age 31. In those few cases where this difference in risks shrinks and, ultimately, turns to zero, the underlying reason is not a true smoothing of risks across children with parents' differing in their wageincome levels but rather a statistical property following from too few children with high-income parents showing up in this particular situation later in life. 


\subsubsection{Main findings}

These results on the link between the child's probability of showing up in alternative labour market situations later in life and the parents' educational and income background show, first and foremost, that each parental dimension - the mother's and the father's educational background and the parents' wage-income level - mostly retains an independent and significant relation to the child's later labour market outcomes also after accounting for other background factors, notably the child's early school-to-work-transition experiences. Indeed, an independent relation for each of the three parental dimensions shows up, in most cases, even though they are accounted for jointly, that is, with all three indicators included at the same time. Moreover, the parents' educational background and their wage-income level tell slightly different stories, implying that accounting for both of them produces a fuller picture of the link between the child's family background and later labour market outcomes.

The country-specific results in relation to the child's probability of continuing in education when aged 21, 26 and 31 indicate the following. The probability of the child studying when aged 21 increases with the parents' educational level and also with their wage-income level. The same pattern appears five years later, at age 26 , and still ten years later, at age 31 , but with the link turning increasingly weaker.

A similar pattern shows up also when it comes to the link between the child's later employment probability and the parents' educational and income status. In particular, a higher educational level of the parents is linked to a lower probability of the child of moving into working life rather than continuing in education, whereas a higher wage-income level of the parents tends to improve the employment prospects of the child in adulthood.

The link between the child's risk of ending up in NEET activities later in life and the parents' educational and income background is, on average, notably weaker and also much more dispersed, when compared to the corresponding link for the child's educational and employment activities. The link to the parents' educational and income background for the child's risk of experiencing unemployment when aged 21 resembles that observed for the child's educational and employment probabilities: the link is negative, strengthens with the parents' education and income, but weakens when the child grows older. Moreover, the child's unemployment risk seems to be more strongly linked to the parents' income level than to their educational level, whereas the opposite holds typically true for the child's educational and employment probabilities. In other 
words, the child's risk of experiencing unemployment in adulthood is strongest for children with low-income parents, although this relation tends to weaken with age.

Also the child's risk of moving into disability arrangements or other types of inactivity when aged 21, or beyond, seems to be more strongly linked to the parents' wage-income level than to their educational background. In the case of disability benefits, the negative link (i.e. lower probability) tends to strengthen with the parents' educational and income levels: the risk is highest for children with low-education/lowincome parents and lowest for children with high-education/highincome parents. For other types of inactivity, this trend of a steadily strengthening link depending on the child's family background is mostly missing in the sense that the main difference occurs between loweducation/low-income parents, on the one hand, and highereducation/higher-income parents, on the other hand. Another conspicuous difference between these two inactivity categories is that the childparent link strengthens for disability benefits but weakens for other (unknown) types of inactivity when the child approaches the age of 31 .

Finally, it is worth noting that our results indicate that there is no major difference between the role of the mother's and the father's educational background: the overall pattern is basically the same when it comes to both the sign and the strength of the investigated child-parent links. Another noteworthy feature is that there are more clear-cut differences in the strength of these links in relation to parents' educational background than in relation to their wage-income levels. More precisely, there are distinct differences in the child's labour market outcome probabilities later in life when comparing children with low-educated parents to children with higher educated parents, as well as when comparing children with secondary-level educated parents to children with tertiary-level educated parents. In relation to parents' wage-income levels, in contrast, the main and often only difference occurs between children with low-income parents and children with higher-income parents. In other words, the differences between children with middleincome and high-income parents are often minor or negligible. 


\subsection{Early pathways through education and labour market outcomes}

The other major set of background factors included in our statistical model concerns what we have labelled "stylized" school-to-work pathways for those young people who have achieved no post-compulsory degree by the time they turn 21 . Several recent studies have explored the relationship between non-completion of upper secondary school and later labour market outcomes [e.g. Bäckman et al. (2011) for Scandinavia, and Bratsberg et al. (2010) and Falch and Nyhus (2011) for Norway]. In this section, we go one step further and investigate this relationship by means of early school-to-work pathways. These stylized pathways - 16 in total - are defined and discussed in Chapter 5 and will, therefore, not be subject to a detailed presentation in this context. The results reported below refer to those obtained when also accounting for gender, cohort and family background. As became evident already in the previous sub-chapter when discussing the role of family background, these early school-to-work trajectories are closely related to the education and income levels of parents. Accordingly we have reason to keep this in mind also when exploring the pathway results. However, even after accounting for differences in family background, early school-towork-transition experiences often prove to be closely linked to the young person's later labour market outcomes. In other words, good or bad early post-compulsory-school experiences may strengthen or weaken the role of family background.

The extent to which different school and labour market experiences early in life tend to increase or decrease young people's probability of showing up in alternative labour market situations is the main topic of this sub-chapter. Since our results cover four countries, five alternative labour market outcomes, three age points $(21,26$ and 31$)$ at which these outcomes are projected, and a total of 16 stylized school-to-work pathways, the most efficient way of reporting this multidimensional amount of results is not self-evident. All dimensions have their own value. However, as the focus is on the 16 stylized pathways, they are also chosen as our point of departure for the categorisation of the results to be reported next. In other words, for each stylized pathway we will show the probability of young people following that particular pathway of being in either one of the five main activity categories investigated when aged 21, 26 and 31 , respectively.

Evidently, it is not possible or even meaningful to comment in detail on this multitude of results. Instead, the text will mainly focus on disen- 
tangling overall patterns and trends, while country-specific results and peculiarities are easily identified in the separate tables and, therefore, mostly overlooked when commenting on key results. In the section "Main findings" concluding this sub-chapter, an attempt will be made to summarize all these results from an "opposite" perspective, viz. from the view of young peoples' probabilities of showing up in the five alternative labour market situations given the stylized pathway followed straight after completion of compulsory school.

\subsubsection{Early post-compulsory-school pathways: continue in education}

As noted in Chapter 5, large shares of the youngsters identified as noncompleters at age 21 have continued in education straight after completing compulsory school and have also stayed as full-time students over the next five years, at least. But despite this unbroken record of study years, they have not succeeded in completing an upper secondary degree by the time they turn 21 . This holds true also for another stylized pathway, the dominant feature of which is a delayed start in postcompulsory education due to a break year spent outside both education and the labour market (in unknown activities), upon which follows an unbroken record of years enrolled in full-time education. Next, we look in more detail into the link between these two education-dominated pathways and the probability of young people having followed such pathways of being in the five different labour market situations when aged 21,26 and 31 , respectively.

We start by reporting the results for the early post-compulsoryschool trajectory representing an unbroken record of study years from age 16 up to age 20 [11111; with 1 standing for full-time student]. As shown in Chapter 5, the share of young non-completers following this stylized pathway - or highly similar pathways - is close to $35 \%$ for Denmark, about 30\% for Norway, some 29\% for Sweden but less than $22 \%$ for Finland. Table 7.7 a gives the differences in the probability of these particular non-completers, when compared to completers, of being in the five alternative labour market situations at the three different age points investigated. 
Table 7.7a: "Study-track" non-completers' probabilities in terms of labour market outcomes at age 21, 26 and 31, respectively, by country; differences in probabilities compared to completers

\begin{tabular}{|c|c|c|c|c|}
\hline Labour market outcome & $\begin{array}{r}\text { Denmark } \\
\text { [11111] } \\
\text { share } 34.9 \%\end{array}$ & $\begin{array}{r}\text { Finland } \\
\text { [11111] } \\
\text { share } 21.6 \%\end{array}$ & $\begin{array}{r}\text { Norway } \\
\text { [11111] } \\
\text { share } 30.3 \%\end{array}$ & $\begin{array}{r}\text { Sweden } \\
\text { [11111] } \\
\text { share } 29.2 \%\end{array}$ \\
\hline \multicolumn{5}{|l|}{ Full-time student } \\
\hline age 21 & 0.296 & 0 & -0.044 & 0 \\
\hline age 26 & -0.126 & -0.028 & -0.093 & 0 \\
\hline age 31 & 0 & 0 & 0 & 0.027 \\
\hline \multicolumn{5}{|l|}{ Employed } \\
\hline age 21 & -0.307 & -0.042 & -0.034 & -0.140 \\
\hline age 26 & 0.057 & -0.093 & 0 & -0.124 \\
\hline age 31 & -0.107 & -0.117 & -0.067 & -0.139 \\
\hline \multicolumn{5}{|l|}{ Unemployed } \\
\hline age 21 & 0 & 0 & 0.045 & 0.121 \\
\hline age 26 & 0.032 & 0.059 & 0.041 & 0.064 \\
\hline age 31 & 0 & 0.047 & 0.034 & 0.043 \\
\hline \multicolumn{5}{|l|}{ Disability beneficiary } \\
\hline age 21 & 0 & 0.007 & 0.024 & 0.011 \\
\hline age 26 & 0.005 & 0.019 & 0.036 & 0.028 \\
\hline age 31 & 0.021 & 0.034 & 0.038 & 0.042 \\
\hline \multicolumn{5}{|l|}{ Other (inactive) } \\
\hline age 21 & 0.012 & 0.055 & 0.010 & 0.014 \\
\hline age 26 & 0.032 & 0.044 & 0.019 & 0.027 \\
\hline age 31 & 0.068 & 0.036 & 0 & 0.027 \\
\hline
\end{tabular}

Notes: The reported average probabilities are obtained after also accounting for gender, cohort and family background. A negative sign implies a weaker probability of being in that particular activity, when compared to completers, that is, those having achieved a post-compulsory degree by the time they turn 21. Low (high) absolute values indicate a small (large) difference with respect to completers. All reported probabilities are significant at the $99 \%$ level or more. Insignificant and only weakly significant probabilities are set at zero. The probabilities are calculated from pooled information on the three youth cohorts under study. For definitions of the five main activity groups, see Chapter 2 . For a definition and discussion of the 16 stylized post-compulsory-school pathways up to age 20 constructed for the Nordic non-completers, see Chapter 5. The percentage share for the pathway in question among the non-completers, as reported for each country, is taken from Chapter 5. "1" refers to being a full-time student.

The corresponding results for non-completers having delayed their start in upper secondary education by one year after completion of compulsory school [51111; with 1 standing for full-time student and 5 for inactivity ("other")] are shown in Table 7.7b. While the share of non-completers following this type of pathway is notably lower compared to the "studytrack" non-completers, it is far from negligible: $4.1 \%$ for Denmark, $6.2 \%$ for Norway, $7.7 \%$ for Sweden and as high as $13.5 \%$ for Finland. 
Table 7.7b "Delayed-study-track" non-completers' probabilities in terms of labour market outcomes at age 21, 26 and 31, respectively, by country; differences in probabilities compared to completers

\begin{tabular}{|c|c|c|c|c|}
\hline Labour market outcome & $\begin{array}{r}\text { Denmark } \\
\text { [51111] } \\
\text { share } 4.1 \%\end{array}$ & $\begin{array}{r}\text { Finland } \\
\text { [51111] } \\
\text { share } 13.5 \%\end{array}$ & $\begin{array}{r}\text { Norway } \\
\text { [51111] } \\
\text { share } 6.2 \%\end{array}$ & $\begin{array}{r}\text { Sweden } \\
\text { [51111] } \\
\text { share } 7.7 \%\end{array}$ \\
\hline \multicolumn{5}{|l|}{ Full-time student } \\
\hline age 21 & 0.199 & -0.027 & -0.059 & 0.038 \\
\hline age 26 & -0.108 & -0.047 & -0.138 & -0.033 \\
\hline age 31 & 0 & 0 & 0 & 0 \\
\hline \multicolumn{5}{|l|}{ Employed } \\
\hline age 21 & -0.275 & -0.096 & -0.078 & -0.235 \\
\hline age 26 & -0.022 & -0.172 & 0 & -0.159 \\
\hline age 31 & -0.111 & -0.216 & -0.139 & -0.180 \\
\hline \multicolumn{5}{|l|}{ Unemployed } \\
\hline age 21 & 0.022 & 0.014 & 0.057 & 0.125 \\
\hline age 26 & 0.052 & 0.101 & 0.063 & 0.088 \\
\hline age 31 & 0 & 0.075 & 0.051 & 0.056 \\
\hline \multicolumn{5}{|l|}{ Disability beneficiary } \\
\hline age 21 & 0.003 & 0.020 & 0.039 & 0.053 \\
\hline age 26 & 0.010 & 0.052 & 0.060 & 0.065 \\
\hline age 31 & 0 & 0.075 & 0.069 & 0.085 \\
\hline \multicolumn{5}{|l|}{ Other (inactive) } \\
\hline age 21 & 0.051 & 0.089 & 0.040 & 0.019 \\
\hline age 26 & 0.069 & 0.066 & 0.031 & 0.038 \\
\hline age 31 & 0.059 & 0.079 & 0.032 & 0.026 \\
\hline
\end{tabular}

Notes: See Table 7.7a above. " 1 " refers to being a full-time student, " 5 " to belonging to the category "other" (inactivity).

The beyond-age-20 studying behaviour of study-track and delayedstudy-track non-completers is quite similar. In both Finland and Sweden, these two non-completer groups appear to have an, at most, only slightly lower probability, when compared to completers, of pursuing full-time studies also after age 20. In Norway, on the other hand, both noncompleter groups have a much lower probability of being in full-time education still at age 21 , with this difference with respect to completers widening further up to age 26 . Moreover, the delayed-study-track noncompleters seem to be in a weaker position in this respect than are the study-track non-completers. For Denmark, there is no clear-cut agerelated trend discernible: non-completers have a much higher studying probability at age 21 , but a much lower studying probability at age 26 , when compared to young people having graduated from upper secondary school already by age 21 . Moreover, while Danish study-track noncompleters are more likely to continue in education at age 21 than are their delayed-study-track non-completer peers, the situation is reversed at age 26.

Hence, this cross-country picture looks quite messy with an overwhelming dominance of dissimilarities rather than similarities. Ultimately, this is only to be expected, though, in view of highly different 
country rates of young non-completers, late completers and adult noncompleters (cf. Chapter 6) in combination with distinct differences in post-compulsory education systems. By age 31 , however, these differences across the four Nordic countries in relation to study-dominated tracks have disappeared.

The most conspicuous pattern when it comes to beyond-age- 20 employment probabilities is that completers are much more likely to be employed at age 21 than are study-track non-completers, with the employment probability typically being even lower for delayed-study-track non-completers. Moreover, this pattern is, by and large, repeated at all three age points investigated. For Finland, we observe a clear-cut trend in this respect with the employment probability of these non-completer groups weakening considerably with age. A similar age-related pattern is not discernible for the other three countries.

The counterpart to these differences in employment probabilities is a much lower unemployment risk among completers than among both study-track and delayed-study-track non-completers, with the latter group of non-completers again doing worse than their study-track noncompleter peers. However, this difference with respect to completers in unemployment risks tends to narrow with age, a pattern that is discernible in all four countries and most clearly for Sweden.

The risk of becoming a disability beneficiary is notably higher for delayed-study-track non-completers, less so for study-track noncompleters, when compared to completers. Moreover, this risk is increasing with age, more strongly for delayed-study-track noncompleters than for study-track non-completers. This overall pattern shows up in all four countries. The cross-country pattern is more dispersed when it comes to other (unknown) types of inactivity. Studytrack and especially delayed-study-track non-completers encounter a higher risk than completers also in this respect, but the age-related trend varies a lot across the four countries: in Denmark it is increasing and in Finland decreasing with age. These differences in risks are, on average, smaller in Norway and Sweden than in Denmark and Finland, revealing no age-related trend whatsoever.

\subsubsection{Early post-compulsory-school pathways: move into working life at a young age}

The analysis in Chapter 5 of non-completers' stylized post-compulsoryschool trajectories revealed that large shares of them drop out from upper secondary education and move into working life, so it seems, on 
quite a permanent basis. This dropout pattern is particularly common among those having continued in upper secondary education for two or three years. It is less frequent among young non-completers having dropped out already after one year, as well as among those who never continued in education after compulsory school. Moreover, although they tend to spend most of their time after school dropout in employment, their employment profile is of a much more bumpy nature, when compared to that of non-completers dropping out only after two or three years in upper secondary education.

Next we investigate to what extent these employment-dominated post-compulsory-school pathways of non-completers are related to their probability of ending up in the five main labour market situations under scrutiny 5, 10 and 15 years after completion of compulsory education. Again, the group of reference is completers, that is, those young people having achieved an upper secondary degree by the time they turn 21 . We thereby start with the most typical early employment tracks followed by non-completers, viz. tracks characterised by the young noncompleter continuing directly in post-compulsory education for two or three years before substituting school with work, but without having graduated from upper secondary education [11222 and 11122; with 1 standing for full-time student and 2 for employed].

As indicated in Chapter 5, the share of non-completers following these two types of early employment pathways is close to $24 \%$ for Norway and only slightly lower for Denmark (22\%), Sweden (21\%) and Finland (about 20\%). These four Nordic countries also share the feature of the 3-year-study-employment track covering a clearly larger share of the non-completers than the 2-year-study-employment track, except in Denmark where the latter track is slightly more common. Table 7.8a presents the differences for the relevant probabilities, when compared to the situation of completers, for non-completers leaving upper secondary education for work after three years of full-time studies. The corresponding information for non-completers dropping out for employment already after two years of full-time studies is given in Table 7.8b.

Not surprisingly, young non-completers moving early into working life are less likely than completers to show up as full-time students also beyond age 20. In all four countries, this lower studying probability, when compared to completers, is highest at age 21 , considerably lower at age 26 and almost non-existent at age 31. In view of the results presented in Chapter 2, this time trend obviously follows from increasing numbers of completers moving into working life rather than from early dropouts in working life returning to full-time education. Interestingly, both the pat- 
tern and strength of this lower studying probability are highly similar for non-completers staying either two or three years in upper secondary education before dropping out. Hence, it does not seem to matter that much whether the young non-completer has left upper secondary education after two or only after three years of full-time studies.

Table 7.8a: "Dropout-after-three-years-employment-track" non-completers' probabilities in terms of labour market outcomes at age 21, 26 and 31, respectively, by country; differences in probabilities when compared to completers

\begin{tabular}{|c|c|c|c|c|}
\hline Labour market outcome & $\begin{array}{r}\text { Denmark } \\
\text { [11122] } \\
\text { share } 10.3 \%\end{array}$ & $\begin{array}{r}\text { Finland } \\
\text { [11122] } \\
\text { share } 11.1 \%\end{array}$ & $\begin{array}{r}\text { Norway } \\
\text { [11122] } \\
\text { share } 14.6 \%\end{array}$ & $\begin{array}{r}\text { Sweden } \\
\text { [11122] } \\
\text { share } 13.8 \%\end{array}$ \\
\hline \multicolumn{5}{|l|}{ Full-time student } \\
\hline age 21 & -0.234 & -0.294 & -0.406 & -0.248 \\
\hline age 26 & -0.119 & -0.081 & -0.114 & -0.089 \\
\hline age 31 & 0.031 & 0 & -0.029 & 0 \\
\hline \multicolumn{5}{|l|}{ Employed } \\
\hline age 21 & 0.149 & 0.266 & 0.290 & 0.204 \\
\hline age 26 & 0.033 & 0.043 & 0 & 0.026 \\
\hline age 31 & -0.095 & 0 & -0.067 & -0.066 \\
\hline \multicolumn{5}{|l|}{ Unemployed } \\
\hline age 21 & 0.028 & 0 & 0.058 & 0.045 \\
\hline age 26 & 0.033 & 0.021 & 0.047 & 0.041 \\
\hline age 31 & 0 & 0 & 0.041 & 0.030 \\
\hline \multicolumn{5}{|l|}{ Disability beneficiary } \\
\hline age 21 & 0 & -0.003 & 0.011 & 0 \\
\hline age 26 & 0.005 & 0 & 0.015 & 0.009 \\
\hline age 31 & 0.014 & 0 & 0.029 & 0.023 \\
\hline \multicolumn{5}{|l|}{ Other (inactive) } \\
\hline age 21 & 0.055 & 0.028 & 0.047 & 0 \\
\hline age 26 & 0.043 & 0 & 0.031 & 0.013 \\
\hline age 31 & 0.039 & 0 & 0.026 & 0.016 \\
\hline
\end{tabular}

Notes: See Table 7.7a above. "1" refers to being a full-time student, " 2 " to being employed.

Likewise, it is not surprising to find that non-completers moving early into working life tend to have a higher probability of being employed also at age 21, when compared to completers. This advantage in terms of employment prospects vanishes rapidly, though. In all four countries, the employment probability of these non-completers is at age 26 only slightly higher than among completers and by age 31 , it has turned negative in all countries except Finland. In other words, young completers tend to rapidly close the employment gap to non-completers having moved into working life at an early age. Already by age 31, the employment prospects of completers are clearly better. Again, we see small, if any, differences in these respects between non-completers having left upper secondary education after two years compared to those having dropped out only after three years. 


\begin{tabular}{|c|c|c|c|c|}
\hline Labour market outcome & $\begin{array}{r}\text { Denmark } \\
\text { [11222] } \\
\text { share } 11.8 \%\end{array}$ & $\begin{array}{r}\text { Finland } \\
\text { [11222] } \\
\text { share } 8.8 \%\end{array}$ & $\begin{array}{r}\text { Norway } \\
\text { [11222] } \\
\text { share } 9.4 \%\end{array}$ & $\begin{array}{r}\text { Sweden } \\
\text { [11222] } \\
\text { share } 7.4 \%\end{array}$ \\
\hline \multicolumn{5}{|l|}{ Full-time student } \\
\hline age 21 & -0.205 & -0.294 & -0.418 & -0.224 \\
\hline age 26 & -0.131 & -0.105 & -0.194 & -0.070 \\
\hline age 31 & 0 & 0 & -0.043 & 0 \\
\hline \multicolumn{5}{|l|}{ Employed } \\
\hline age 21 & 0.119 & 0.256 & 0.293 & 0.169 \\
\hline age 26 & 0.037 & 0.039 & 0.078 & 0 \\
\hline age 31 & -0.080 & 0 & -0.053 & -0.061 \\
\hline \multicolumn{5}{|l|}{ Unemployed } \\
\hline age 21 & 0.030 & 0 & 0.063 & 0.055 \\
\hline age 26 & 0.040 & 0.022 & 0.051 & 0.036 \\
\hline age 31 & 0.019 & 0 & 0.030 & 0.026 \\
\hline \multicolumn{5}{|l|}{ Disability beneficiary } \\
\hline age 21 & 0.002 & 0 & 0.016 & 0 \\
\hline age 26 & 0.008 & 0 & 0.027 & 0.015 \\
\hline age 31 & 0.013 & 0 & 0.031 & 0.024 \\
\hline \multicolumn{5}{|l|}{ Other (inactive) } \\
\hline age 21 & 0.053 & 0.030 & 0.047 & 0 \\
\hline age 26 & 0.047 & 0.042 & 0.038 & 0.013 \\
\hline age 31 & 0.039 & 0 & 0.035 & 0.020 \\
\hline
\end{tabular}

Notes: See Table 7.7a above. "1" refers to being a full-time student, " 2 " to being employed.

The probability of these early-working non-completers of encountering unemployment at age 21 , or beyond, is only slightly higher compared to the situation faced by their completer peers. Moreover, this pattern of relatively small differences in unemployment risks, when compared to completers, holds true at all three age points investigated and, indeed, shows up in all four countries. Also in this respect, the situation is much the same for young non-completers moving into employmentdominated tracks after three years or already after two years in upper secondary education.

The two tables also indicate that these non-completers have an only a marginally, if any, higher probability of going into disability arrangements after having turned 20, when compared to completers. For Finland, there are no significant differences in this respect between completers and non-completers having moved early into working life. In the other three countries, the difference is small, albeit weakly increasing with age. Again, the pattern is strikingly similar for those dropping out either after two or only after three years in upper secondary education.

When it comes to other types of inactivity, the disadvantage in relation to completers is clearly stronger, especially in Denmark and Norway. However, this higher risk of non-completers having entered work- 
ing life at a young age of ending up in inactivity declines with age, implying that the difference with respect to completers is typically smaller at age 31 than at age 21 .

On the whole, then, we identify relatively small differences in the risk of showing up in NEET activities between completers and noncompleters moving, so it seems, quite successfully into working life already at a young age. This finding is most likely related to their comparatively good employment prospects, when compared to completers, also later in life. Hence, by means of a close to finished upper secondary education coupled with early engagement in working life, they seem to have been able to achieve competencies that keep them employed also in adulthood. Moreover, in this respect we see no major differences between non-completers dropping out after two or only after three years in upper secondary education.

Next we compare the outcome of these two groups of noncompleters to those of their non-completer peers having dropped out already after one or no year in post-compulsory education. More precisely, this first post-compulsory-school year is typically spent either in education [12222] or outside both education and the labour market [52222], with 1 standing for full-time student, 2 for employment and 5 for "other" (inactivity). The results for both of these groups are included in Table 7.8c for the simple reason that the two groups are so small for Norway that it is not possible to obtain separate results for them at age 21 . Indeed, also when taken together, they cover less than $4 \%$ of the Norwegian non-completers. Their combined share is slightly higher in Sweden (about 5\%) and highest in Denmark $(13.6 \%)$ and Finland (10.6\%). However, while those dropping out from school already after one year represent the dominating track (close to 10\%) of the two in Denmark, the "non-starters" make up a larger share (about 6\%) in Finland.

As is also to be expected, the studying probability of these noncompleters looks much the same as the studying probability of noncompleters dropping out slightly later. All four groups of non-completers substitute school with work at a young age, implying that they are much less likely than their completer peers to be enrolled in education especially when comparing their situation at age 21 . With increasing numbers of also completers moving into working life, the difference in studying probabilities between completers and non-completers narrows rapidly and, ultimately, disappears. 
Table 7.8c: "Drop-out-after-one-year-employment-track" \& “Inactivity-year-employment-track" non-completers' probabilities in terms of labour market outcomes at age 21, 26 and 31, respectively, by country; differences in probabilities when compared to completers

\begin{tabular}{|c|c|c|c|c|c|c|c|c|}
\hline \multirow[b]{2}{*}{$\begin{array}{l}\text { Labour market } \\
\text { outcome }\end{array}$} & \multicolumn{2}{|c|}{ Denmark } & \multicolumn{2}{|c|}{ Finland } & \multicolumn{2}{|c|}{ Norway } & \multicolumn{2}{|c|}{ Sweden } \\
\hline & $\begin{array}{r}\text { [12222] } \\
\text { Share } \\
9.7 \%\end{array}$ & $\begin{array}{r}\text { [52222] } \\
\text { share } \\
3.9 \%\end{array}$ & $\begin{array}{r}\text { [12222] } \\
\text { share } \\
4.4 \%\end{array}$ & $\begin{array}{r}\text { [52222] } \\
\text { share } \\
6.2 \%\end{array}$ & $\begin{array}{r}\text { [12222] } \\
\text { share } \\
2.7 \%\end{array}$ & $\begin{array}{r}\text { [52222] } \\
\text { share } \\
0.9 \%\end{array}$ & $\begin{array}{r}\text { [12222] } \\
\text { share } \\
3.0 \%\end{array}$ & $\begin{array}{r}\text { [52222] } \\
\text { share } \\
2.1 \%\end{array}$ \\
\hline \multicolumn{9}{|c|}{ Full-time student } \\
\hline at age 21 & -0.172 & -0.286 & -0.288 & -0.350 & -0.383 & -0.383 & -0.217 & -0.290 \\
\hline at age 26 & -0.154 & -0.174 & -0.110 & -0.129 & -0.215 & -0.138 & -0.102 & -0.094 \\
\hline at age 31 & 0 & 0 & 0 & 0 & -0.065 & 0 & 0 & 0 \\
\hline \multicolumn{9}{|l|}{ Employed } \\
\hline at age 21 & 0.099 & 0.171 & 0.224 & 0.271 & 0.195 & 0.195 & 0.146 & 0.236 \\
\hline at age 26 & 0.061 & 0.044 & 0 & 0 & 0.070 & 0 & 0 & 0 \\
\hline at age 31 & -0.067 & -0.107 & 0 & 0 & -0.056 & -0.139 & -0.080 & -0.108 \\
\hline \multicolumn{9}{|l|}{ Unemployed } \\
\hline at age 21 & 0.024 & 0.050 & 0 & 0 & 0.065 & 0.065 & 0.047 & 0 \\
\hline at age 26 & 0.034 & 0.043 & 0.026 & 0.032 & 0.066 & 0.063 & 0.038 & 0.041 \\
\hline at age 31 & 0.013 & 0 & 0 & 0 & 0.047 & 0.051 & 0 & 0.042 \\
\hline \multicolumn{9}{|c|}{ Disability beneficiary } \\
\hline at age 21 & 0.002 & 0 & 0 & 0 & 0.024 & 0.024 & 0 & 0.017 \\
\hline at age 26 & 0.009 & 0.010 & 0 & 0 & 0.021 & 0.060 & 0 & 0.018 \\
\hline at age 31 & 0.014 & 0.021 & 0 & 0 & 0.028 & 0.069 & 0 & 0 \\
\hline \multicolumn{9}{|l|}{ Other (inactive) } \\
\hline at age 21 & 0.047 & 0.062 & 0.048 & 0.058 & 0.099 & 0.099 & 0 & 0.020 \\
\hline at age 26 & 0.049 & 0.078 & 0.047 & 0.054 & 0.059 & 0.031 & 0.050 & 0.031 \\
\hline at age 31 & 0.033 & 0.068 & 0 & 0 & 0.046 & 0.032 & 0 & 0 \\
\hline
\end{tabular}

Notes: See Table 7.7a above. " 1 " refers to being a full-time student, " 2 " to being employed and " 5 " to belonging to "other" (inactivity). For Norway, the distribution across the five labour market outcomes of non-completers following an early post-compulsory-school trajectory of [12222] or [52222] is too skewed at age 21 to allow separate probabilities to be estimated for the two pathways.

Also with respect to the probability of being employed still beyond age 20 , the overall pattern is highly similar for the four groups of noncompleters following early employment-dominated tracks. More precisely, all of them tend to have a higher probability of being employed also at age 21, when compared to completers. Another common feature of these four non-completer groups is that this advantage in terms of employment prospects vanishes rapidly with age. In all four countries, they have when aged 26 an employment probability that is only slightly, if at all, higher than for completers. By age 31, this advantage has turned into a disadvantage in all countries except Finland. However, despite this common overall pattern there are also distinct differences between the four groups of early-employment non-completers, the most conspicuous being the following: young non-completers not having continued in postcompulsory education have, when aged 31, notably weaker employment prospects not only when compared to completers but also when compared to their non-completer peers having continued in postcompulsory education for at least one year. In Norway, for instance, 
these non-starters' employment probability at age 31 is $14 \%$ lower than for completers and about 8\% lower than for their dropout noncompleter peers.

When it comes to NEET activities, similar overall patterns show again up for all four non-completer groups following early employmentdominated tracks. Broadly speaking, all of them face a higher risk of ending up in NEET activities, when compared to completers. However, as is evident in Table 7.8c illustrating the outcome for non-completers entering working life after only one or no year in post-compulsory education, the results with respect to NEET activities are occasionally surrounded by uncertainty. Compared to the results reported in Tables 7.8a and $7.8 \mathrm{~b}$, this shows up in less clear-cut age-related trends. More important, it is reflected in a considerable number of cells containing zeros, indicating no difference in probabilities when compared to completers, when the correct interpretation rather is that the number of completers and/or non-completers in that particular labour market situation is too small for producing reliable (robust) results. But also with this warning in mind, the labour market outcome probabilities reported in the three tables seem to strongly suggest that young non-starters in upper secondary education fare much worse as young adults also when compared to their working peers having dropped out early from upper secondary education. Moreover, this contention is well in line with the results reported in the previous chapter.

\subsubsection{Early post-compulsory-school pathways: unemployment experiences at a young age}

In our national datasets, "unemployment" covers situations where the young person is registered as an unemployed jobseeker. This means that we occasionally observe quite large differences across the four Nordic countries in the shares of young people in unemployment, but also in the cross-cohort trend in these shares within single countries. This holds true for those below the age of 25 and even more so for those below the age of 21. Needless to say, a major reason for these observations is differences between countries and changes within countries in the preconditions for being eligible for unemployment benefits in combination with potential differences between countries and changes within countries in the registering behaviour of particularly those young people not eligible for receiving unemployment benefits: Will they still register as unemployed?

In Chapter 5, we constructed for the non-completers a total of three stylized post-compulsory-school trajectories dominated by unemploy- 
ment experiences at a young age. These tracks are identical to the employment-dominated tracks discussed above in the sense that they start with a varying number of years in post-compulsory schooling before dropping out without graduating. However, now a dropout after one, two or three years in post-compulsory schooling turns into early years dominated by unemployment. Next, we present and comment on the labour market outcomes up to age 31 that are most probably related to dropping out from school and experiencing prolonged unemployment already before the age of 21 .

Again, we start by first presenting results for the most typical track: non-completers who continue directly in post-compulsory education but drop out after three years of full-time studies just to end up in (registered) unemployment [11133; with 1 standing for full-time student and 3 for unemployment]. Based on the information given in Chapter 5 , the share of non-completers in our three youth cohorts following this type of unemployment-dominated pathway is $2.1 \%$ for Denmark, 3.5\% for Finland, $4.5 \%$ for Norway and $11.6 \%$ for Sweden. Table 7.9 a reports the differences for the relevant probabilities when compared to the situation of completers.

\begin{tabular}{|c|c|c|c|c|}
\hline Labour market outcome & $\begin{array}{r}\text { Denmark } \\
\text { [11133] } \\
\text { share } 2.1 \%\end{array}$ & $\begin{array}{r}\text { Finland } \\
\text { [11133] } \\
\text { share } 3.5 \%\end{array}$ & $\begin{array}{r}\text { Norway } \\
\text { [11133] } \\
\text { share } 4.5 \%\end{array}$ & $\begin{array}{r}\text { Sweden } \\
\text { [11133] } \\
\text { share } 11.6 \%\end{array}$ \\
\hline \multicolumn{5}{|l|}{ Full-time student } \\
\hline age 21 & -0.119 & -0.181 & -0.295 & -0.129 \\
\hline age 26 & -0.124 & -0.068 & -0.164 & -0.048 \\
\hline age 31 & 0 & 0 & -0.049 & 0 \\
\hline \multicolumn{5}{|l|}{ Employed } \\
\hline age 21 & -0.173 & -0.068 & 0.094 & -0.147 \\
\hline age 26 & -0.086 & -0.143 & 0 & -0.151 \\
\hline age 31 & -0.236 & -0.172 & -0.111 & -0.198 \\
\hline \multicolumn{5}{|l|}{ Unemployed } \\
\hline age 21 & 0.141 & 0.128 & 0.102 & 0.225 \\
\hline age 26 & 0.062 & 0.113 & 0.086 & 0.107 \\
\hline age 31 & 0.037 & 0.090 & 0.069 & 0.074 \\
\hline \multicolumn{5}{|l|}{ Disability beneficiary } \\
\hline age 21 & 0.015 & 0 & 0.028 & 0.011 \\
\hline age 26 & 0.049 & 0 & 0.041 & 0.040 \\
\hline age 31 & 0.066 & 0 & 0.041 & 0.059 \\
\hline \multicolumn{5}{|l|}{ Other (inactive) } \\
\hline age 21 & 0.135 & 0.118 & 0.072 & 0.040 \\
\hline age 26 & 0.098 & 0.083 & 0.060 & 0.052 \\
\hline age 31 & 0.081 & 0 & 0.050 & 0.053 \\
\hline
\end{tabular}

Notes: See Table 7.7a above. "1" refers to being a full-time student, “3” to being unemployed. 
The pattern in relation to the probability of showing up as a full-time student at age 21, 26 and/or 31 is identical to that observed for young non-completers substituting school with work: a much lower probability of being enrolled in full-time education at age 21, with this difference with respect to completers typically levelling out with age. Dropout noncompleters with early unemployment experiences also tend to encounter much lower employment possibilities. These notably weaker employment prospects are discernible already at age 21 . Moreover, they tend to weaken further with age, being considerably weaker at age 31 than at age 21. Conversely, their risk of continuing in or returning to unemployment is obvious. Indeed, they encounter still at age 31 a risk of becoming unemployed that is higher than that faced by completers, but also higher than the risk of becoming unemployed characterising noncompleters having substituted school with work at an early age.

When it comes to other types of NEET activities, we observe initially small but increasing risks of these non-completers moving into disability arrangements. This pattern shows up in all countries except Finland. Non-completers following unemployment tracks after having dropped out after three years in upper secondary education also have a remarkably high risk of being in other (unknown) types of inactivity already when aged 21. While this risk tends to decline with age it is, nonetheless, relatively high still at age 31. In Denmark, for instance, it is almost $12 \%$ higher than for completers and, indeed, also much higher than for noncompleters having dropped out from school into working life.

Dropping out into unemployment after three years in upper secondary education without graduation thus tends to result in a risky labour market situation also as a young adult. In particular, the employment prospects are much weaker and the risk of ending up in NEET activities much higher than for completers, but also much higher than for noncompleter peers having successfully entered the labour market despite dropping out from upper secondary education. Moreover, this weaker situation typically prevails still 15 years after completion of compulsory school. These findings thus lend further support to the international literature in this field.

Next, we compare this outcome for young unemployed noncompleters having dropped out after three years of upper secondary education to that of their unemployed peers having dropped out already after two years or after only one year in post-compulsory education. Table $7.9 \mathrm{~b}$ reports the results obtained for non-completers who continued in post-compulsory education but dropped out after two years of full-time studies just to end up in unemployment [11333; with 1 stand- 
ing for full-time student and 3 for unemployment]. The share of noncompleters following this type of pathway is of much the same size as for the [11133] pathway, but only in Denmark and Finland. For Norway it is slightly lower and for Sweden notably lower.

Table 7.9b: "Dropout-after-two-years-unemployment-track" non-completers' probabilities in terms of labour market outcomes at age 21, 26 and 31, respectively, by country; differences in probabilities when compared to completers

\begin{tabular}{|c|c|c|c|c|}
\hline Labour market outcome & $\begin{array}{r}\text { Denmark } \\
\text { [11333] } \\
\text { share } 1.8 \%\end{array}$ & $\begin{array}{r}\text { Finland } \\
\text { [11333] } \\
\text { share } 3.7 \%\end{array}$ & $\begin{array}{r}\text { Norway } \\
{[11333]} \\
\text { share } 3.0 \%\end{array}$ & $\begin{array}{r}\text { Sweden } \\
\text { [11333] } \\
\text { share } 4.2 \%\end{array}$ \\
\hline \multicolumn{5}{|l|}{ Full-time student } \\
\hline age 21 & -0.202 & -0.197 & -0.399 & -0.112 \\
\hline age 26 & -0.158 & -0.074 & -0.218 & -0.060 \\
\hline age 31 & 0 & 0 & 0 & 0 \\
\hline \multicolumn{5}{|l|}{ Employed } \\
\hline age 21 & -0.157 & -0.087 & 0.150 & -0.188 \\
\hline age 26 & -0.136 & -0.188 & 0 & -0.190 \\
\hline age 31 & -0.214 & -0.174 & -0.137 & -0.224 \\
\hline \multicolumn{5}{|l|}{ Unemployed } \\
\hline age 21 & 0.159 & 0.128 & 0.104 & 0.212 \\
\hline age 26 & 0.088 & 0.138 & 0.089 & 0.136 \\
\hline age 31 & 0 & 0.109 & 0.072 & 0.081 \\
\hline \multicolumn{5}{|l|}{ Disability beneficiary } \\
\hline age 21 & 0.037 & 0 & 0.029 & 0.018 \\
\hline age 26 & 0.076 & 0 & 0.040 & 0.050 \\
\hline age 31 & 0.039 & 0.026 & 0.047 & 0.073 \\
\hline \multicolumn{5}{|l|}{ Other (inactive) } \\
\hline age 21 & 0.162 & 0.151 & 0.115 & 0.069 \\
\hline age 26 & 0.130 & 0.111 & 0.073 & 0.064 \\
\hline age 31 & 0.118 & 0.062 & 0.037 & 0.058 \\
\hline
\end{tabular}

Notes: See Table 7.7a above. "1" refers to being a full-time student, “3” to being unemployed.

Table 7.9c, finally, reports the probability of ending up in alternative labour market situations for non-completers starting an unemployment track record already after one year in post-compulsory education [13333, with 1 standing for full-time student, 3 for unemployment]. As is evident in the table, the share of non-completers in our data following such tracks is mostly very low: $0.7 \%$ for Denmark, $0.8 \%$ for Sweden, $1.9 \%$ for Norway but as high as $5.1 \%$ for Finland. 
Table 7.9c: "Dropout-after-one-year-unemployment-track" non-completers' probabilities in terms of labour market outcomes at age 21, 26 and 31, respectively, by country; differences in probabilities when compared to completers

\begin{tabular}{|c|c|c|c|c|}
\hline Labour market outcome & $\begin{array}{r}\text { Denmark } \\
\text { [13333] } \\
\text { share } 0.7 \%\end{array}$ & $\begin{array}{r}\text { Finland } \\
\text { [13333] } \\
\text { share } 5.1 \%\end{array}$ & $\begin{array}{r}\text { Norway } \\
\text { [13333] } \\
\text { share } 1.9 \%\end{array}$ & $\begin{array}{r}\text { Sweden } \\
\text { [13333] } \\
\text { share } 0.8 \%\end{array}$ \\
\hline \multicolumn{5}{|l|}{ Full-time student } \\
\hline age 21 & -0.211 & -0.167 & -0.349 & -0.193 \\
\hline age 26 & -0.106 & -0.105 & -0.174 & -0.076 \\
\hline age 31 & 0 & 0 & 0 & 0 \\
\hline \multicolumn{5}{|l|}{ Employed } \\
\hline age 21 & -0.146 & -0.108 & 0.091 & -0.197 \\
\hline age 26 & -0.178 & -0.202 & 0 & -0.192 \\
\hline age 31 & -0.181 & -0.255 & -0.162 & -0.259 \\
\hline \multicolumn{5}{|l|}{ Unemployed } \\
\hline age 21 & 0.131 & 0.109 & 0.112 & 0.196 \\
\hline age 26 & 0.130 & 0.174 & 0.095 & 0.106 \\
\hline age 31 & 0 & 0.117 & 0.082 & 0.091 \\
\hline \multicolumn{5}{|l|}{ Disability beneficiary } \\
\hline age 21 & 0 & 0.006 & 0.029 & 0.042 \\
\hline age 26 & 0 & 0.023 & 0.041 & 0.073 \\
\hline age 31 & 0 & 0.033 & 0.050 & 0.081 \\
\hline \multicolumn{5}{|l|}{ Other (inactive) } \\
\hline age 21 & 0.211 & 0.160 & 0.118 & 0.152 \\
\hline age 26 & 0.122 & 0.110 & 0.101 & 0.089 \\
\hline age 31 & 0.090 & 0.123 & 0.060 & 0.091 \\
\hline
\end{tabular}

Notes: See Table 7.7a above. "1" refers to being a full-time student and " 3 " to being unemployed.

By and large, the same overall pattern is repeated for all three groups of non-completers with early experiences from (registered) unemployment. This contention seems to hold true despite the uncertainty evidently surrounding the results reported in Table $7.9 \mathrm{c}$ due to small shares of non-completers following such tracks (except for Finland). In sum, the picture mediated by the three tables indicates the following. The probability of these young non-completers of being enrolled in fulltime education when aged 21 is low, but this difference with respect to completers diminishes with graduated young people moving increasingly from education into working life. All these non-completers going early into unemployment also face a much lower probability of being employed by age 21 , and beyond, with their employment prospects worsening further with age.

A comparison of the three tables further suggests that the picture outlined above tends to strengthen when going from young unemployed non-completers having dropped out only after three years in upper secondary education to those having dropped out already after one year in post-compulsory education. However, such a pattern appears only in relation to education and employment probabilities. When it comes to the risk of experiencing unemployment also in adulthood, there are mi- 
nor differences between these three groups of young unemployed noncompleters differing in their years in post-compulsory education before dropout. Indeed, this seems to hold true also with respect to the risk of moving on disability benefits, whereas the risk of ending up in other (unknown) types of inactivity reveals a tendency of typically being the higher, the earlier the young non-completer has dropped out from school into unemployment. Finally, while the three tables indicate the difference in probabilities when compared to completers, a comparison of Tables 7.9a-c and Tables 7.8a-c suggests that young non-completers with early experiences from unemployment fare, on average, worse also when compared to young non-completers skipping school for work.

\subsubsection{Early post-compulsory-school pathways: becoming a young disability beneficiary}

When it comes to young disability beneficiaries, we refer to a recent analysis of ours (Albæk et al., 2014) published in the final report of the so-called NORWELL project funded by the Nordic Council of Ministers. Nonetheless, we will use some space also in this context to highlight labour market outcomes of young adults having experienced health problems at an early age. Doing so, we are able to obtain a fuller picture of the role of all the 16 stylized pathways presented and discussed in Chapter 5 in terms of non-completers' labour market situation as young adults. Moreover, the Albæk et al. (2014) analysis did not cover Sweden, merely Denmark, Finland and Norway.

As for the employment and unemployment pathways discussed above, we concentrate on early tracks starting with a varying number of years in post-compulsory schooling before ending with the young person dropping out, now to become a disability beneficiary. The total share of non-completers in these types of pathways is low relative to the shares of non-completers following the other stylized pathways - close to $1 \%$ for Denmark, 3\% for Finland, about 2\% for Norway and $4 \%$ for Sweden - but far from negligible in absolute terms.

The first stylized pathway analysed below turns into a disabilitybenefit track after three years in post-compulsory schooling straight after completion of basic education [11144; with 1 standing for full-time student and 4 for disability beneficiary]. The share of young non-completers following such pathways is $0.1 \%$ for Denmark, $0.5 \%$ for Finland, $1.5 \%$ for Norway and $1 \%$ for Sweden. Table 7.10a gives the differences for the relevant probabilities when compared to the situation of completers. 
Table 7.10a: "After-three-years-dropout-disability-track" non-completers' probabilities in terms of labour market outcomes at age 21, 26 and 31, respectively, by country; differences in probabilities when compared to completers

\begin{tabular}{|c|c|c|c|c|}
\hline Labour market outcome & $\begin{array}{r}\text { Denmark } \\
{[11144]} \\
\text { share } 0.1 \%\end{array}$ & $\begin{array}{r}\text { Finland } \\
\text { [11144] } \\
\text { share } 0.5 \%\end{array}$ & $\begin{array}{r}\text { Norway } \\
\text { [11144] } \\
\text { share } 1.5 \%\end{array}$ & $\begin{array}{r}\text { Sweden } \\
\text { [11144] } \\
\text { share } 1.0 \%\end{array}$ \\
\hline \multicolumn{5}{|l|}{ Full-time student } \\
\hline age 21 & -0.427 & -0.378 & -0.188 & -0.172 \\
\hline age 26 & -0.330 & -0.152 & 0 & -0.107 \\
\hline age 31 & -0.097 & 0 & 0 & 0 \\
\hline \multicolumn{5}{|l|}{ Employed } \\
\hline age 21 & -0.440 & -0.287 & -0.109 & -0.384 \\
\hline age 26 & -0.353 & -0.526 & -0.160 & -0.580 \\
\hline age 31 & 0 & -0.504 & -0.182 & -0.753 \\
\hline \multicolumn{5}{|l|}{ Unemployed } \\
\hline age 21 & -0.025 & -0.061 & -0.139 & -0.038 \\
\hline age 26 & -0.033 & 0 & 0.107 & 0 \\
\hline age 31 & -0.016 & 0 & 0.072 & -0.021 \\
\hline \multicolumn{5}{|l|}{ Disability beneficiary } \\
\hline age 21 & 0.945 & 0.702 & -0.074 & 0.612 \\
\hline age 26 & 0.716 & 0.599 & 0.099 & 0.728 \\
\hline age 31 & 0 & 0.501 & 0.120 & 0.760 \\
\hline \multicolumn{5}{|l|}{ Other (inactive) } \\
\hline age 21 & -0.052 & 0 & -0.084 & -0.018 \\
\hline age 26 & 0 & 0 & 0 & 0.026 \\
\hline age 31 & -0.027 & 0 & 0 & -0.023 \\
\hline
\end{tabular}

Notes: See Table 7.7a above. "1" refers to being a full-time student, " 4 " to being a disability beneficiary. For Norway, the distribution across the five labour market outcomes of non-completers following an early post-compulsory-school trajectory of [11144], [11444] or [14444] is too skewed at age 21 to allow separate probabilities to be estimated for these three pathways at this particular age, which explains the parentheses surrounding the estimated difference in probabilities for alternative labour market outcomes at age 21 .

Young non-completers moving into disability arrangements after having dropped out from three years of full-time studies at an upper secondary school reveal a low probability of having returned to school by age 21 . They also have a low probability of being employed at this particular age, with their employment prospects weakening further with age. The only exception is Denmark, but due to the low share of non-completers in this type of track, the Danish results should in this context be interpreted with caution. Generally non-completers having followed this type of track also tend to face a slightly higher risk of being unemployed, when compared to completers, but this pattern varies a lot between countries and also within countries with age. Additionally, they typically have a high risk, also when compared to other young non-completers, of continuing on or returning to disability benefits as young adults, whereas their likelihood of ending up in other types of inactivity is mostly very low.

In the next table (Table 7.10b), we turn the focus to young disability beneficiaries having dropped out after two years or already after one 
year in post-compulsory schooling [11444 and 14444; with 1 standing for full-time student and 4 for being a disability beneficiary]. The share of non-completers following either one of these two pathways is comparatively high only in two cases, both of which cover immediate or almost immediate moves into disability arrangements after completion of compulsory school [the 14444 track]: $2.3 \%$ for Finland and 2.8\% for Sweden, with the rest of shares staying below $0.5 \%$.

Table 7.10b: "Dropout-after-two-years-disability-track" and "Dropout-after-one-year-disabilitytrack" non-completers' probabilities in terms of labour market outcomes at age 21, 26 and 31, respectively, by country; differences in probabilities when compared to completers

\begin{tabular}{|c|c|c|c|c|c|c|c|c|}
\hline \multirow[b]{2}{*}{$\begin{array}{l}\text { Labour market } \\
\text { outcome }\end{array}$} & \multicolumn{2}{|c|}{ Denmark } & \multicolumn{2}{|c|}{ Finland } & \multicolumn{2}{|c|}{ Norway } & \multicolumn{2}{|c|}{ Sweden } \\
\hline & $\begin{array}{r}{[11444]} \\
\text { share } \\
0.4 \%\end{array}$ & $\begin{array}{r}{[14444]} \\
\text { share } \\
0.4 \%\end{array}$ & $\begin{array}{r}\text { [11444] } \\
\text { share } \\
0.2 \%\end{array}$ & $\begin{array}{r}{[14444]} \\
\text { share } \\
2.3 \%\end{array}$ & $\begin{array}{r}\text { [11444] } \\
\text { share } \\
0.3 \%\end{array}$ & $\begin{array}{r}\text { [14444] } \\
\text { share } \\
0.1 \%\end{array}$ & $\begin{array}{r}{[11444]} \\
\text { share } \\
0.2 \%\end{array}$ & $\begin{array}{r}\text { [14444] } \\
\text { share } \\
2.8 \%\end{array}$ \\
\hline \multicolumn{9}{|l|}{$\begin{array}{l}\text { Full-time stu- } \\
\text { dent }\end{array}$} \\
\hline at age 21 & -0.439 & -0.439 & -0.346 & -0.419 & -0.188 & -0.188 & -0.293 & -0.379 \\
\hline at age 26 & -0.320 & -0.330 & -0.192 & -0.234 & 0 & 0 & 0 & -0.196 \\
\hline at age 31 & 0 & 0 & -0.132 & -0.118 & 0 & & 0 & -0.058 \\
\hline \multicolumn{9}{|l|}{ Employed } \\
\hline at age 21 & -0.395 & -0.407 & -0.295 & -0.356 & -0.109 & -0.109 & -0.450 & -0.459 \\
\hline at age 26 & 0 & -0.483 & -0.556 & -0.602 & -0.468 & 0 & -0.604 & -0.626 \\
\hline at age 31 & 0 & 0 & -0.607 & -0.736 & 0 & 0 & -0.639 & -0.812 \\
\hline \multicolumn{9}{|l|}{ Unemployed } \\
\hline at age 21 & -0.025 & -0.025 & -0.070 & -0.078 & -0.139 & -0.139 & -0.054 & -0.070 \\
\hline at age 26 & -0.033 & -0.033 & 0 & -0.053 & 0.098 & 0 & 0 & -0.039 \\
\hline at age 31 & 0 & 0 & -0.049 & -0.038 & 0 & 0 & -0.021 & -0.016 \\
\hline \multicolumn{9}{|l|}{$\begin{array}{l}\text { Disability } \\
\text { beneficiary }\end{array}$} \\
\hline at age 21 & 0.911 & 0.923 & 0.720 & 0.883 & -0.074 & -0.074 & 0.831 & 0.942 \\
\hline at age 26 & 0 & 0.871 & 0.715 & 0.925 & 0.105 & 0 & 0.652 & 0.891 \\
\hline at age 31 & 0 & 0 & 0.839 & 0.933 & 0.118 & 0.118 & 0.660 & 0.908 \\
\hline \multicolumn{9}{|l|}{ Other (inactive) } \\
\hline at age 21 & -0.052 & -0.052 & 0 & -0.030 & -0.084 & -0.084 & -0.035 & -0.034 \\
\hline at age 26 & -0.025 & -0.025 & 0 & -0.036 & 0.132 & 0 & 0 & -0.032 \\
\hline at age 31 & 0 & 0 & -0.050 & -0.041 & 0 & 0 & 0 & -0.021 \\
\hline
\end{tabular}

Notes: See Table 7.10a above.

The overall impression arising from comparing the labour market prospects of the three groups of young non-completers experiencing health problems at an early age is that their situation looks much the same, despite the uncertainty undeniably surrounding many of the results due to small numbers of observations fulfilling particular combinations of background characteristics. In other words, it does not seem to matter how many years the young person has spent in upper secondary education before dropping out without achieving a degree. In all three cases, the most likely outcome is that the young person continues in or returns to disability arrangements also in adulthood. 


\subsubsection{Early post-compulsory-school pathways: withdrawal at young age into inactivity}

The last set of our 16 stylized pathways constructed for the noncompleters contains a total of four early post-compulsory-school trajectories dominated by years spent outside both education and the labour force, that is, in activities not covered by any of the large administrative registers from which our datasets are compiled. These "other" situations may, as noted in previous chapters, imply engagement in highly different activities which may have a positive influence on the young person's near-future choices, but may also involve activities starting a risky pathway into adulthood.

The first three pathways are characterised by the young noncompleter having continued directly in education after completing compulsory school. Their main difference lies in the number of years enrolled in full-time education before dropping out: after three years, after two years or already after one post-compulsory education year. Taken together, these three pathways cover $16.3 \%$ of the non-completers in Denmark, 11.3\% in Finland, 22.8\% in Norway and 10.3\% in Sweden. In other words, a substantial number of young people drop out from school just to withdraw from taking part in educational or in labour market activities. The fourth pathway, in contrast, involves no post-compulsory education whatsoever: it starts with the young person withdrawing straightaway after leaving compulsory school from both education and the labour market, and continues with the person staying outside any such activities up to age 20, at least. The share of young non-completers following such tracks is low in Norway (1.7\%) and Denmark (3.5\%) but worryingly high in both Finland (8\%) and Sweden (7\%).

Next we look somewhat more in detail into the labour market outcomes that these withdrawal pathways seem to be most strongly related to. We thereby start with the pathway preceded by three years in postcompulsory education before dropout [11155; with 1 standing for fulltime student and 5 for "other" (activities)]. The share of non-completers following this type of early trajectory is strikingly high in all four Nordic countries under study: $6.4 \%$ for Denmark, $7.4 \%$ for Finland, $13.3 \%$ for Norway and $6.1 \%$ for Sweden. The probability of ending up in alternative labour market situations in adulthood is, therefore, of particular interest for this comparatively large group of non-completers. These probabilities, as compared to completers, are displayed in Table 7.11a.

Table 7.11a shows that young non-completers withdrawing into inactivity after having dropped out from upper secondary education after three years of full-time studies, are unlikely to have returned to educa- 
tion by age 21 . Although this difference in studying probabilities, when compared to completers, shrinks with age, this narrowing is, once again, obviously mainly due to completers having left the education system rather than these dropouts having returned to education by age 26 or by age 31 . They are also much less likely to be in employment at age 21 , with their employment prospects weakening rather than improving with age. Probably, this also explains their typically only slightly higher risk, when compared to completers, of being registered as unemployed jobseekers as young adults. Moreover, their risk of moving into disability arrangements increases with age, especially in Denmark and Sweden. Finally, they are very likely to continue in inactivity still at age 21 , with this risk declining only slowly with age.

Table 7.11a: "Dropout-after-three-years-withdrawal-track" non-completers' probabilities in terms of labour market outcomes at age 21, 26 and 31, respectively, by country; differences in probabilities when compared to completers

\begin{tabular}{|c|c|c|c|c|}
\hline Labour market outcome & $\begin{array}{r}\text { Denmark } \\
\text { [11155] } \\
\text { share } 6.4 \%\end{array}$ & $\begin{array}{r}\text { Finland } \\
{[11155]} \\
\text { share } 7.4 \%\end{array}$ & $\begin{array}{r}\text { Norway } \\
\text { [11155] } \\
\text { share } 13.3 \%\end{array}$ & $\begin{array}{r}\text { Sweden } \\
{[11155]} \\
\text { share } 6.1 \%\end{array}$ \\
\hline \multicolumn{5}{|l|}{ Full-time student } \\
\hline age 21 & -0.164 & -0.248 & -0.373 & -0.207 \\
\hline age 26 & -0.074 & -0.049 & -0.083 & 0 \\
\hline age 31 & 0.058 & 0 & 0 & 0 \\
\hline \multicolumn{5}{|l|}{ Employed } \\
\hline age 21 & -0.160 & -0.097 & 0.149 & -0.153 \\
\hline age 26 & -0.132 & -0.182 & -0.072 & -0.192 \\
\hline age 31 & -0.236 & -0.184 & -0.157 & -0.170 \\
\hline \multicolumn{5}{|l|}{ Unemployed } \\
\hline age 21 & 0.057 & 0.027 & 0.068 & 0.113 \\
\hline age 26 & 0.059 & 0.100 & 0.063 & 0.053 \\
\hline age 31 & 0.024 & 0.074 & 0.051 & 0 \\
\hline \multicolumn{5}{|l|}{ Disability beneficiary } \\
\hline age 21 & 0.015 & 0.014 & 0.025 & 0.034 \\
\hline age 26 & 0.045 & 0.017 & 0.032 & 0.075 \\
\hline age 31 & 0.073 & 0.030 & 0.042 & 0.103 \\
\hline \multicolumn{5}{|l|}{ Other (inactive) } \\
\hline age 21 & 0.252 & 0.304 & 0.132 & 0.213 \\
\hline age 26 & 0.103 & 0.114 & 0.060 & 0.086 \\
\hline age 31 & 0.081 & 0.085 & 0.053 & 0.048 \\
\hline
\end{tabular}

Notes: See Table 7.7a above. "1" refers to being a full-time student, " 5 " to being in "other" activities.

The corresponding differences in probabilities, as compared to completers, for those young non-completers withdrawing into inactivity after two years in post-compulsory education [11555; with 1 standing for fulltime student and 5 for "other" (activities)] are reported in Table 7.11b. The share of non-completers following this type of track is less than $2.5 \%$ in both Finland and Sweden, about twice as large for Denmark and, again, highest (6\%) for Norway. 
Table 7.11b: "Dropout-after-two-years-withdrawal-track" non-completers' probabilities in terms of labour market outcomes at age 21, 26 and 31, respectively, by country; differences in probabilities when compared to completers

\begin{tabular}{|c|c|c|c|c|}
\hline Labour market outcome & $\begin{array}{r}\text { Denmark } \\
\text { [11555] } \\
\text { share } 4.9 \%\end{array}$ & $\begin{array}{r}\text { Finland } \\
\text { [11555] } \\
\text { share } 2.2 \%\end{array}$ & $\begin{array}{r}\text { Norway } \\
\text { [11555] } \\
\text { share } 6.0 \%\end{array}$ & $\begin{array}{r}\text { Sweden } \\
\text { [11555] } \\
\text { share } 2.4 \%\end{array}$ \\
\hline \multicolumn{5}{|l|}{ Full-time student } \\
\hline age 21 & -0.165 & -0.220 & -0.341 & -0.158 \\
\hline age 26 & -0.122 & -0.069 & -0.111 & 0 \\
\hline age 31 & 0.051 & 0 & 0 & 0 \\
\hline \multicolumn{5}{|l|}{ Employed } \\
\hline age 21 & -0.232 & -0.192 & 0.090 & -0.262 \\
\hline age 26 & -0.176 & -0.281 & -0.098 & -0.270 \\
\hline age 31 & -0.290 & -0.200 & -0.163 & -0.291 \\
\hline \multicolumn{5}{|l|}{ Unemployed } \\
\hline age 21 & 0.078 & 0 & 0.077 & 0.138 \\
\hline age 26 & 0.073 & 0.112 & 0.081 & 0.064 \\
\hline age 31 & 0.023 & 0 & 0.059 & 0.071 \\
\hline \multicolumn{5}{|l|}{ Disability beneficiary } \\
\hline age 21 & 0.054 & 0.022 & 0.027 & 0.058 \\
\hline age 26 & 0.097 & 0 & 0.037 & 0.083 \\
\hline age 31 & 0.125 & 0 & 0.042 & 0.100 \\
\hline \multicolumn{5}{|l|}{ Other (inactive) } \\
\hline age 21 & 0.266 & 0.357 & 0.146 & 0.224 \\
\hline age 26 & 0.128 & 0.211 & 0.091 & 0.132 \\
\hline age 31 & 0.092 & 0 & 0.079 & 0.074 \\
\hline
\end{tabular}

Notes: See Table 7.7a above. " 1 " refers to being a full-time student, " 5 " to being in "other" activities.

Based on Table 7.11b, the following may be concluded. Also young noncompleters withdrawing into inactivity already after two years in upper secondary education face a much lower probability of being enrolled in education at age 21, when compared to completers. The magnitude of this lower studying probability and its age-related trend are almost identical to those observed for young non-completers dropping out from upper secondary education only after three years, just to withdraw into inactivity. However, when it comes to their employment prospects, the situation looks notably weaker. In particular, also when compared to their non-completer peers withdrawing only after three years in upper secondary education, their employment prospects are much weaker already when aged 21, with the trend pointing to a faster weakening with age. Also their risk of becoming unemployed is clearly higher. The same pattern is repeated when it comes to their risk of continuing in or returning to inactivity. Moreover, it holds true also in relation to disability benefits, but only for Denmark. In the other three countries, these two groups of early withdrawing non-completers have an approximately equally high risk, when compared to completers, of ending up in disability arrangements. All in all, the overall impression is that young noncompleters dropping out from upper secondary education into inactivity 
fare in most respects worse if dropping out already after two years instead of dropping out only after three years.

The share of non-completers having dropped out from school into inactivity already after having continued in post-compulsory education for only one year [15555; with 1 standing for full-time student and 5 for "other" (activities)] is smaller than for the other two groups of young withdrawing non-completers investigated so far: less than 2\% for Finland and Sweden and 3.5\% for Norway. The only exception is Denmark where this share is approximately the same (5\%) as for the previous group of withdrawing non-completers. These young non-completers' probabilities in relation to later labour market outcomes, when compared to the situation of completers, are given in Table 7.11c.

Table 7.11c: "Dropout-after-one-year-withdrawal-track" non-completers' probabilities in terms of labour market outcomes at age 21, 26 and 31, respectively, by country; differences in probabilities when compared to completers

\begin{tabular}{|c|c|c|c|c|}
\hline Labour market outcome & $\begin{array}{r}\text { Denmark } \\
\text { [15555] } \\
\text { share 5.0\% }\end{array}$ & $\begin{array}{r}\text { Finland } \\
{[15555]} \\
\text { share } 1.7 \%\end{array}$ & $\begin{array}{r}\text { Norway } \\
\text { [15555] } \\
\text { share } 3.5 \%\end{array}$ & $\begin{array}{r}\text { Sweden } \\
{[15555]} \\
\text { share } 1.8 \%\end{array}$ \\
\hline \multicolumn{5}{|l|}{ Full-time student } \\
\hline age 21 & -0.209 & -0.238 & -0.372 & -0.188 \\
\hline age 26 & -0.123 & 0 & -0.158 & 0 \\
\hline age 31 & 0 & 0 & 0 & 0 \\
\hline \multicolumn{5}{|l|}{ Employed } \\
\hline age 21 & -0.221 & -0.143 & 0.095 & -0.261 \\
\hline age 26 & -0.219 & -0.247 & -0.074 & -0.266 \\
\hline age 31 & -0.326 & 0 & -0.195 & -0.295 \\
\hline \multicolumn{5}{|l|}{ Unemployed } \\
\hline age 21 & 0.075 & 0.063 & 0.087 & 0.107 \\
\hline age 26 & 0.075 & 0.126 & 0.078 & 0.102 \\
\hline age 31 & 0 & 0 & 0.072 & 0.064 \\
\hline \multicolumn{5}{|l|}{ Disability beneficiary } \\
\hline age 21 & 0.060 & 0.039 & 0.032 & 0.129 \\
\hline age 26 & 0.095 & 0.023 & 0.040 & 0.076 \\
\hline age 31 & 0.131 & -0.011 & 0.047 & 0.120 \\
\hline \multicolumn{5}{|l|}{ Other (inactive) } \\
\hline age 21 & 0.294 & 0.279 & 0.158 & 0.213 \\
\hline age 26 & 0.171 & 0.138 & 0.113 & 0.128 \\
\hline age 31 & 0.163 & 0 & 0.097 & 0.074 \\
\hline
\end{tabular}

Notes: See Table 7.7a above. "1" refers to being a full-time student, " 5 " to being in "other" activities.

The broad picture emerging based on Table 7.11c is highly similar to that obtained for young withdrawing non-completers having been enrolled for two years in upper secondary education before dropping out. There are, however, a few conspicuous differences. First, in all four countries young withdrawing non-completers dropping out from postcompulsory education already after one year, seem to have an even lower probability of having returned to education by age 21 . Another dis- 
tinct difference compared to young withdrawing non-completers dropping out only after two years, is that their probability of continuing in or returning to inactivity is clearly higher. This holds true for Norway and especially for Denmark, that is, for those two countries where the share of non-completers following this type of inactivity track is highest among the four Nordic countries.

Finally, we turn to the pathway indicating immediate withdrawal after completion of compulsory education [55555; with 5 standing for "other" (activities)]. The share of non-completers following this risky track already before turning 21 is less than $2 \%$ in Norway, $3.5 \%$ in Denmark, but as high as 7\% in Sweden and 8\% in Finland. Table 7.11d gives the differences in probabilities, when compared to completers, of these young non-completers of ending up in alternative labour market situations at age 21,26 and 31 , respectively.

Table 7.11d: "Early-withdrawal-track" non-completers' probabilities in terms of labour market outcomes at age 21, 26 and 31, respectively, by country; differences in probabilities when compared to completers

\begin{tabular}{|c|c|c|c|c|}
\hline Labour market outcome & $\begin{array}{r}\text { Denmark } \\
\text { [55555] } \\
\text { share } 3.5 \%\end{array}$ & $\begin{array}{r}\text { Finland } \\
\text { [55555] } \\
\text { share } 7.9 \%\end{array}$ & $\begin{array}{r}\text { Norway } \\
\text { [55555] } \\
\text { share } 1.7 \%\end{array}$ & $\begin{array}{r}\text { Sweden } \\
\text { [55555] } \\
\text { share } 6.9 \%\end{array}$ \\
\hline \multicolumn{5}{|l|}{ Full-time student } \\
\hline age 21 & -0.244 & -0.244 & -0.426 & -0.254 \\
\hline age 26 & -0.138 & -0.090 & -0.123 & -0.100 \\
\hline age 31 & 0 & -0.051 & 0 & -0.033 \\
\hline \multicolumn{5}{|l|}{ Employed } \\
\hline age 21 & -0.217 & -0.214 & 0.275 & -0.320 \\
\hline age 26 & -0.254 & -0.344 & -0.134 & -0.278 \\
\hline age 31 & -0.444 & -0.343 & -0.185 & -0.372 \\
\hline \multicolumn{5}{|l|}{ Unemployed } \\
\hline age 21 & 0.082 & 0.036 & 0.072 & 0.060 \\
\hline age 26 & 0.074 & 0.138 & 0.090 & 0.040 \\
\hline age 31 & 0 & 0.109 & 0.068 & 0.046 \\
\hline \multicolumn{5}{|l|}{ Disability beneficiary } \\
\hline age 21 & 0.055 & 0.097 & 0 & 0.409 \\
\hline age 26 & 0.094 & 0.068 & 0.047 & 0.246 \\
\hline age 31 & 0.137 & 0.088 & 0.046 & 0.279 \\
\hline \multicolumn{5}{|l|}{ Other (inactive) } \\
\hline age 21 & 0.324 & 0.324 & 0.066 & 0.106 \\
\hline age 26 & 0.224 & 0.228 & 0.120 & 0.092 \\
\hline age 31 & 0.237 & 0.198 & 0.077 & 0.079 \\
\hline
\end{tabular}

Notes: See Table 7.7a above. "5" refers to being in "other" activities.

Young non-completers withdrawing straight after completing compulsory school have a very low probability of being enrolled in education at age 21: compared to young completers, this probability is $43 \%$ lower in Norway and about 25\% lower in the other three countries. Indeed, among the four groups of young non-completers following inactivity- 
dominated tracks early in life, these immediate withdrawers are least likely to have re-entered education by age 21 . They also typically have the weakest employment prospects which, moreover, rapidly weaken further with age: compared to completers, they have in Denmark a $44 \%$ lower probability of being employed when aged 31 . Their risk of becoming unemployed is high, but not necessarily higher than for those withdrawing non-completers having continued in education for at least one year before dropping out.

When it comes to other types of NEET activities the situation is different, though. In particular, among the four groups of withdrawing noncompleters, they have the highest probability of moving into disability arrangements, notably in the two countries with the highest share of young non-completers following this type of track, that is, Finland and Sweden. They also have the highest probability of continuing in or returning to inactivity, but only in Denmark and Finland, whereas this risk is more in line with that of the other three withdrawing groups in Norway and Sweden. However, despite these cross-country differences in certain respects, the overall picture points to these immediate withdrawers having the worst prospects when it comes to labour market outcomes in adulthood. On the whole, it seems that these prospects tend to be the weaker, the earlier the young non-completer withdraws into inactivity.

\subsubsection{Main findings}

This sub-chapter has focused on reporting and discussing results highlighting the potential role of young people's early educational and labour market experiences for their outcomes in adulthood or, more precisely, at age 21, 26 and 31, respectively. These early experiences following upon completion of compulsory school have been approximated by means of 16 stylized pathways constructed for the non-completers and presented in detail in Chapter 5.

With results produced for 16 stylized pathways at three age points and, moreover, separately for four countries, the output from this exercise is quite massive. While the detailed country-specific results have been displayed in a large number of tables, the text surrounding these tables has mainly focused on identifying common patterns among the four Nordic countries, but also distinct differences. However, although there do exist differences across the four countries in certain respects, most of these differences seem to relate to the underlying data. More precisely, since the shares of non-completers going into each of the 16 stylized pathways vary substantially across the four countries, the num- 
ber of observations is not always enough for obtaining robust results, especially as we simultaneously account for differences in gender, cohort and family background. When relevant, such uncertainty related to the presented results has been indicated in the text.

The probabilities of non-completers being in alternative labour market situations at age 21, 26 and 31, respectively, depending on the postcompulsory-school track followed up to age 20 , are throughout contrasted against the situation of completers, that is, young people having graduated from upper secondary education by age 21 . The reported differences in probabilities thus indicate how much more or less likely a non-completer is to show up in a particular labour market situation later in life, when compared to completers. However, comparisons may also be undertaken in other dimensions based on all these results. In particular, it is also possible to compare the outcome across non-completers following different early tracks. While also such attempts were made already when discussing the results displayed in the separate tables, we will conclude this subchapter with four figures which hopefully shed further light on our results and the conclusions to be drawn based on these findings.

The results presented in the subsequent figures are simplified, though, when compared to the detailed tables presented above: the country-specific probabilities have now been turned into Nordic averages with the country-specific shares of non-completers going into each stylized pathway used as weights. This averaging across countries can be justified in view of the similarity in overall patterns. The use of country-specific pathway shares as weights means, in turn, that major results for each non-completer pathway is given more weight. This choice is motivated especially in cases where some country's non-completers are only weakly represented in the pathway in question. On the whole, then, this averaging exercise produces, for each age point, five results for each of the 16 stylized pathways investigated. For instance, we obtain for the non-completers' study-track pathway [11111] five probabilities showing the average difference with respect to completers at age 21 of being (1) enrolled in education; (2) employed; (3) unemployed; (4) on disability benefits; and (5) in other types of inactivity. Corresponding results are produced for this particular pathway also at age 26 and at age 31. Of course, the same exercise is repeated for the other 15 stylized pathways, implying that we now have for each age point only five labour market outcome probabilities per stylized pathway.

We start by presenting these five pathway-specific points for age 21 . This is done in Figure 7.1, but with the early disability-benefit tracks left out and gathered into a separate figure (Figure 7.4). This choice is guid- 
ed by the relatively high probabilities obtained for these tracks: including them with the other stylized pathways, the probabilities of which are of a notably smaller magnitude, would produce a highly unbalanced scatter with most points gathered densely in an unreadable way.

Figure 7.1 clearly shows that non-completers following standard study tracks [11111] are most likely to be enrolled in education also at age 21 . Indeed, they have a higher probability of being in education also when compared to 21-year-old completers (positively signed difference). For those non-completers having spent a year in inactivity before continuing in education [51111], the corresponding difference with respect to completers is minor, albeit still positive. For all other non-completers - that is, those having followed other types of early post-compulsory-school pathways - the probability of being enrolled in full-time education when aged 21 is notably lower (negatively signed difference).

Figure 7.1: Non-completers' probabilities in terms of labour market outcomes at age 21; differences in probabilities, when compared to completers, for 13 stylized pathways as averaged over the four Nordic countries under study

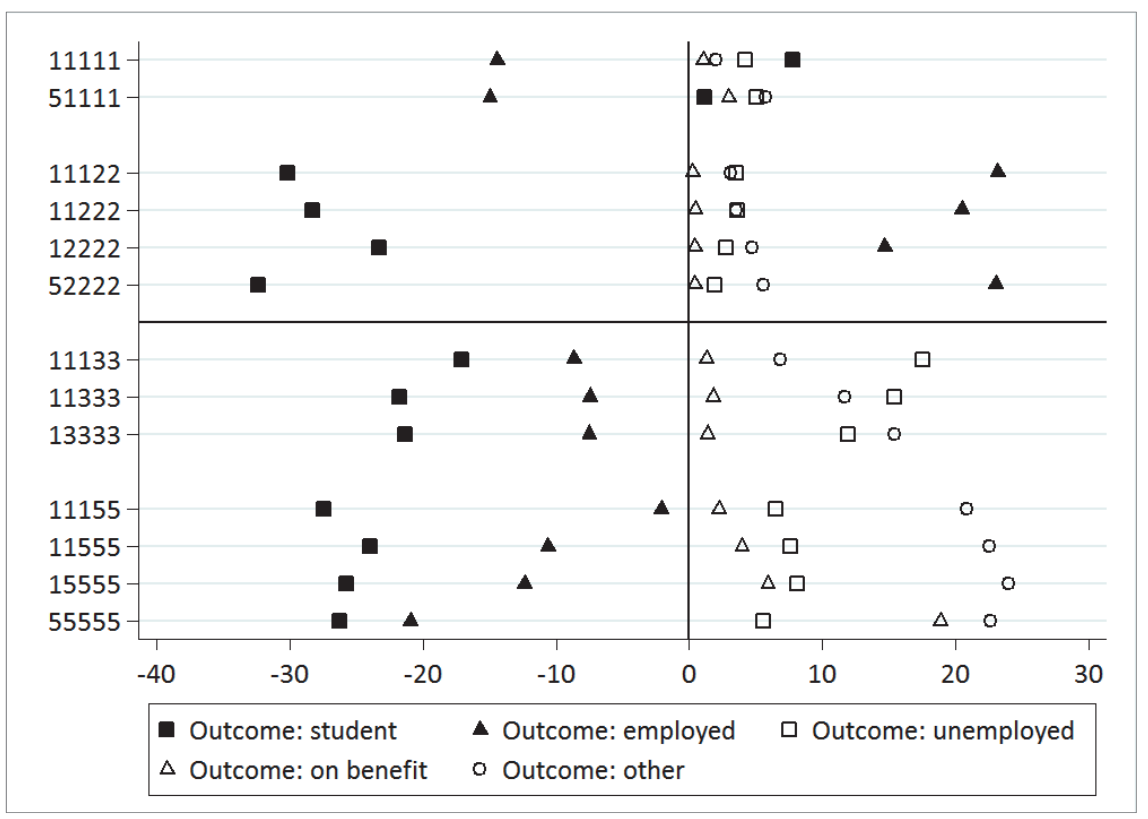

Notes: The figure gives differences in probabilities, when compared to completers, averaged over the four Nordic countries under study. These averages are calculated based on the country-specific probabilities reported in Tables 7.7a to 7.11d, using the country-specific shares of non-completers going into each stylized pathway as weights. The horizontal axis measures these average differences in probabilities with a positive sign indicating a higher probability and a negative sign a lower probability, when compared to completers. The vertical axis represents 13 out of the 16 stylized pathways constructed for Nordic non-completers, as described in Chapter 5. A corresponding presentation for the three stylized disability-benefit pathways is given in Figure 7.4. 
The probability of being employed at age 21 is highest for noncompleters having substituted school with work already at an early age. In other words, young non-completers having started an early and, so it seems, successful employment career appear to experience a clear-cut employment advantage over both completers and non-completers having followed other early tracks, still when aged 21. Indeed, noncompleters having followed other types of early tracks are observed to have the lowest probability not only of being enrolled in education but also of being employed.

The probability of experiencing unemployment when 21 years-of-age is persistently higher for non-completers than for completers irrespective of the early pathway followed by the non-completer. The difference in the risk of showing up as an unemployed jobseeker is especially pronounced for those young non-completers having early unemployment experiences. A similar pattern is observed for young non-completers having withdrawn into inactivity already at an early age in the sense that they face the highest risk of being outside both education and the labour market still when aged 21.

All in all, Figure 7.1 illustrates well that there is a strong link between young people's early and later school and labour market experiences. Moreover, these links do not disappear when also accounting for differences in family background. Indeed, it is worth emphasising also in this context that the differences in probabilities reported in the figure, as well as in the subsequent figures, are those obtained after taking into account differences in parents' educational background and wageincome level. Hence, while differences in family background can go some way in explaining differences in young people's labour market outcomes, there is still an independent role played by their early school and labour market experiences.

The next two figures illustrate the corresponding situation at age 26 (Figure 7.2) and at age 31 (Figure 7.3). The information contained in these two figures indicates that the strong link between early educational and labour market experiences and the probability of being in alternative labour market situations observed at age 21 , is clearly discernible also at age 26 and still at age 31 . 
Figure 7.2: Non-completers' probabilities in terms of labour market outcomes at age 26; differences in probabilities, when compared to completers, for 13 stylized pathways as averaged over the four Nordic countries under study

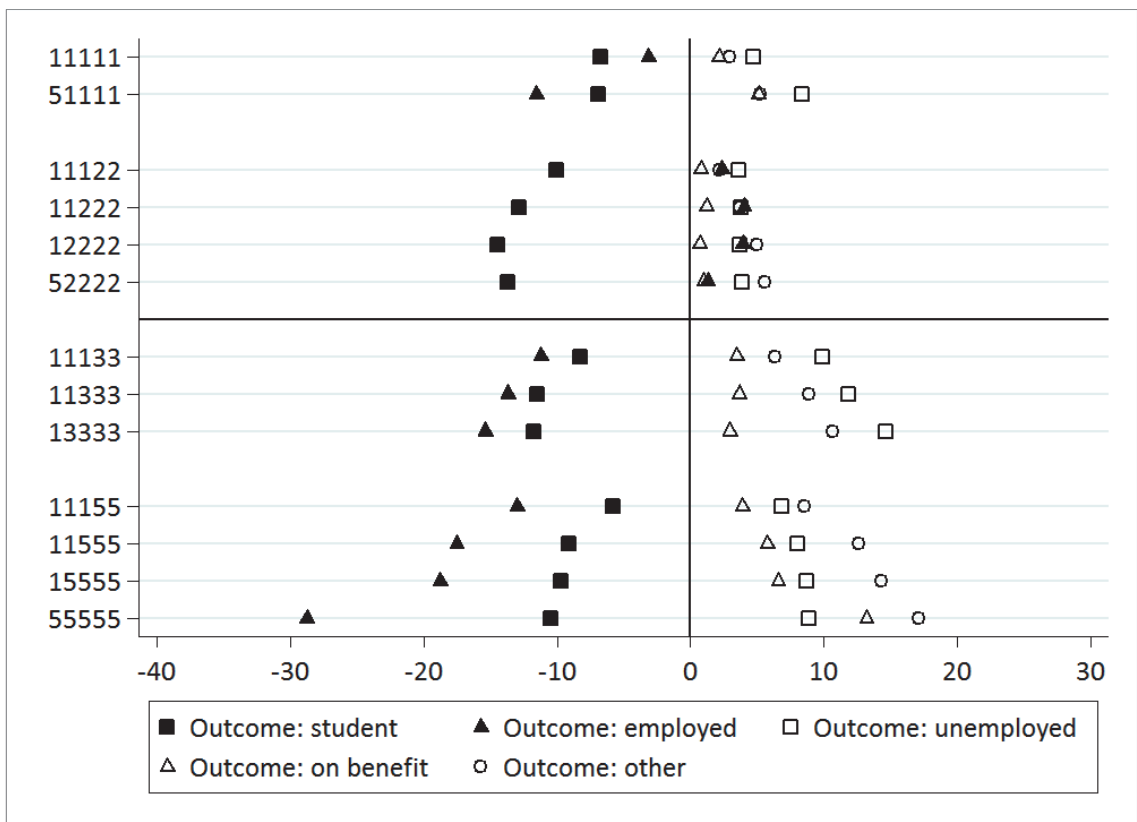

Notes: See Figure 7.1 above.

All early non-completer tracks have, by age 26, resulted in a situation with these young people having a much lower probability of being enrolled as full-time students, when compared to their peers having already by age 21 achieved an upper secondary degree. Noteworthy, however, is that the difference in this probability is somewhat smaller for those non-completers having spent at least three early years in postcompulsory education, but without having completed an upper secondary education still when aged 21 . Non-completers with an early employment track are more likely to be employed than both completers and other non-completers still at age 26 , but with this employment advantage over completers now being notably weaker. The worst employment prospects are faced by young people having withdrawn into inactivity already at an early age.

The situation at age 21 indicated that young non-completers typically face a higher probability, when compared to completers, of being in NEET activities, irrespective of the early track followed. This outcome has not changed by age 26 . On the contrary, it seems to have sharpened further with the probability of ending up as a NEET being the higher the fewer years the young person has attended school. 
Figure 7.3: Non-completers' probabilities in terms of labour market outcomes at age 31; differences in probabilities, when compared to completers, for 13 stylized pathways as averaged over the four Nordic countries under study

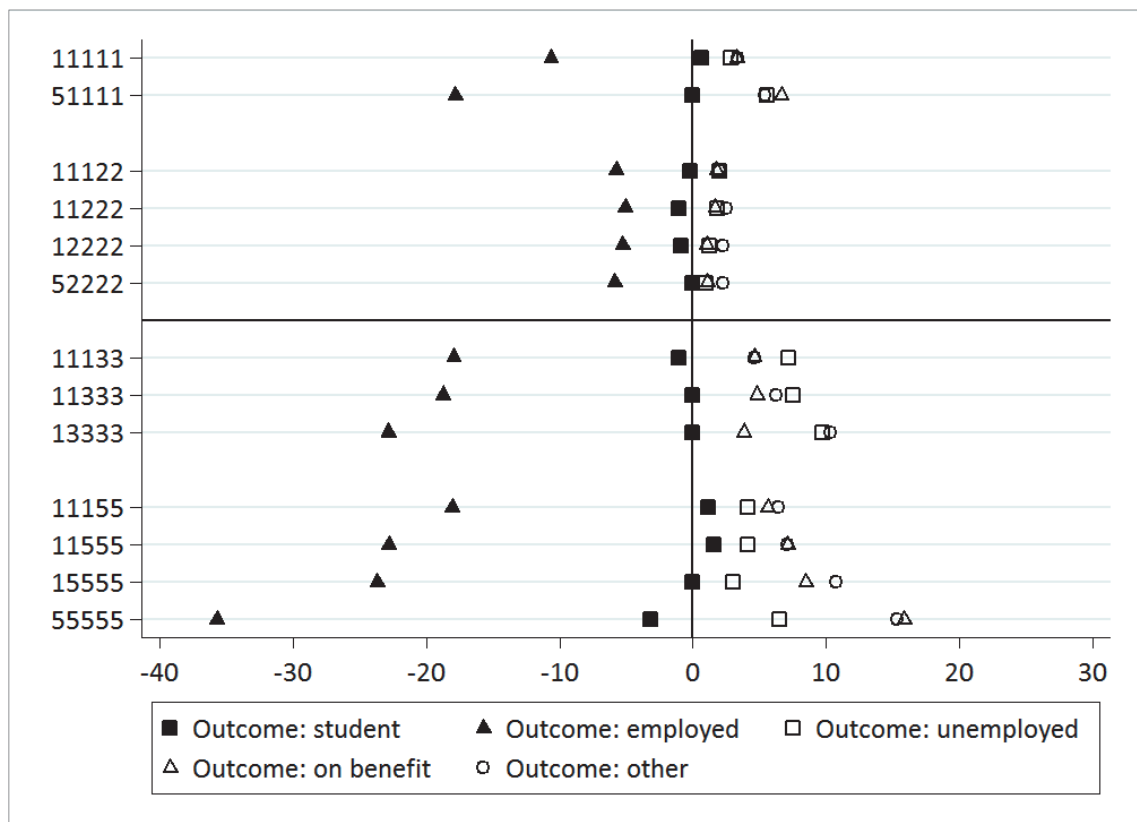

Notes: See Figure 7.1 above.

At age 31, finally, the overall picture looks qualitatively very similar to that observed five years earlier, at age 26 . There have occurred certain interesting changes, though. The employment advantage over completers of those young people having moved early into working life has, by age 31, turned negative. However, this change in employment prospects does not necessarily mean that the employment situation of these young people has weakened in absolute terms. It rather tells that most young completers of upper secondary school have now finished their further education, entered working life and started a successful career. The strong decline up to age 31 in the share of young people being enrolled as full-time students also explains the minor differences in student probabilities irrespective of the early track followed. Young people with early experiences from NEET activities have, also when aged 31, the weakest labour market prospects, albeit the difference with respect to completers is no longer as pronounced at age 31 as at age 26 .

In our last summary figure (Figure 7.4), we illustrate the labour market prospects at age 21, 26 and 31, respectively, of young noncompleters having followed early tracks dominated by time sent on disability benefits. The most conspicuous feature of Figure 7.4 is strong persistence in young people's disability situation: early school leaving, 
with the youngster becoming a disability beneficiary already at a young age, most likely results in the youngster being a disability beneficiary also as a young adult. This persistence, however, should not be interpreted as being due to receiving benefits at an early age, but is rather explained by persistence of the cause behind the young person's early move into disability arrangements.

Figure 7.4: Non-completers' probability of being a disability beneficiary at age 21, 26 and 31, respectively; differences in probability, when compared to completers, for 3 stylized disability-dominated pathways as averaged over the four Nordic countries under study

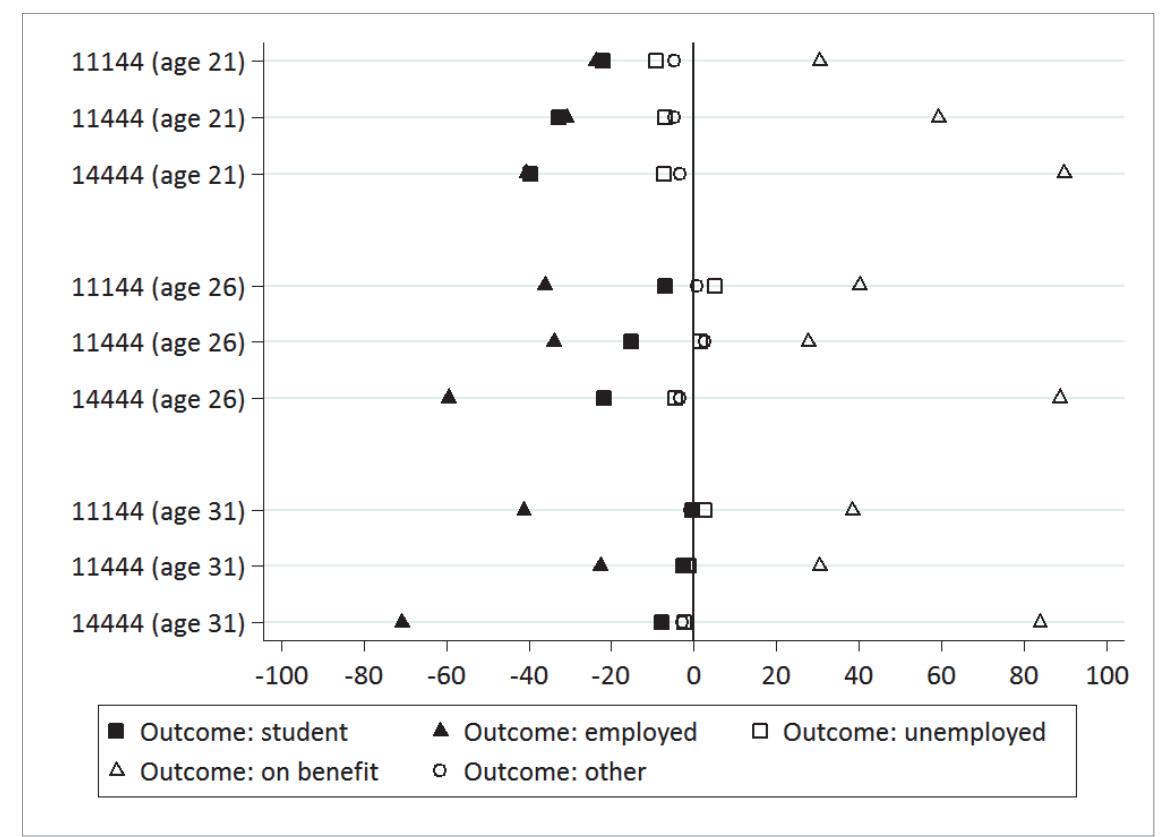

Notes: See Figure 7.1 above.

All in all, a major finding is that we observe growing persistence with age among young people having followed early tracks dominated by NEET activities: early unemployment shows up increasingly in unemployment experiences in adulthood; early disability arrangements tend to continue in adulthood; and early inactivity involves a high risk of continuing outside both education and the labour market, a process often ending with a move into disability arrangements.

However, also study-dominated tracks tend to involve a nonnegligible risk of the non-completer ultimately ending up in NEET activities in adulthood. In view of the results presented in Chapter 6, this outcome is likely to concern especially those young people who fail to com- 
plete an upper secondary degree also beyond the age of 21 , that is, those becoming adult non-completers.

Those who appear to do the best among the non-completers are the ones moving early, without graduating from upper secondary education, but, nonetheless, successfully into working life. An even better solution also for these young non-completers could, of course, be an upper secondary degree before substituting school with work. This would require attractive graduation routes for youth encountering serious problems in finishing their upper secondary education while having a strong desire to work. 



\section{Summary and discussion}

Young people follow highly different trajectories from age 16 up to age 20 , a time period which is often argued to be the most critical in terms of their future labour market outcomes. In other words, the early postcompulsory-school pathway they happen to follow - for one reason or another - is likely to affect their future options and outcomes in a decisive way. The focus of this report has been on investigating the look of these early pathways, as well as on exploring their link to labour market outcomes in adulthood.

In our analyses, we have traced three full cohorts of youth: those who turned 16 in 1993, 1998 and 2003, respectively. All these young people from four Nordic countries - Denmark, Finland, Norway and Sweden have been followed up to the year 2008, implying that the longest follow-up period covers as many as 15 year.

In the Nordic countries, a vast majority of compulsory-school leavers continues immediately, occasionally only after a break year, in upper secondary education and follows up to age 20 , at least, a more or less unbroken track of full-time-study years. However, there are also large shares of young people going into other types of early post-compulsoryschool tracks, many of which involve risky elements such as prolonged withdrawal outside both education and the labour market. In our analyses, we have used so-called clustering techniques to group young people into different clusters depending on the early post-compulsoryschool trajectory followed. (Chapter 3)

Throughout the report, a distinction is made between young people having completed an upper secondary degree by the time they turn 21 (completers), and young people with an exam only from comprehensive school still at age 21 (non-completers). Most of our focus has been on the category of young non-completers. Additionally, attention has been paid to highlighting distinct differences between genders, as well as between the three youth cohorts under scrutiny.

Within the Nordic countries, Denmark and Sweden can be seen to have polar systems of upper secondary schooling, especially when it comes to vocational training. Denmark has a comprehensive apprenticeship system with the young person gathering large amounts of work experience at employers, whereas Sweden can be characterised as hav- 
ing a typical school system also after the reform in 2011. This difference in upper secondary school systems is clearly reflected in young compulsory-school-leavers' trajectories up to age 20 and, hence, also in their completion rates by age. In Sweden, the typical young person continues for three years in upper secondary schooling, with the non-completion rate by 21 being, at most, 17\% in the three youth cohorts investigated. In Denmark, on the other hand, the corresponding non-completion rate is as high as $39 \%$ in the 1998 cohort (and only slightly lower in the other two cohorts) but with the 21-year-old youth being, nonetheless, mostly either in school or in employment. The situation in Norway resembles more that of Denmark than of Sweden, with non-completion rates occasionally reaching $32 \%$. Finland, in turn, looks more like Sweden than like Denmark or Norway, with non-completion rates at age 21 being, at most, close to $20 \%$. (Chapter 2)

Even when restricting the analysis of early post-compulsory-school trajectories to the group of young non-completers, a majority is still found to follow standard study tracks, but without completing an upper secondary degree by age 21 . Indeed, when clustering young Nordic noncompleters into groups common to all four countries, 58\% of Finnish non-completers follow a standard or delayed upper secondary study track, with the corresponding share being about $62 \%$ for Danish noncompleters, some $71 \%$ for Norwegian non-completers and close to $76 \%$ for Swedish non-completers. Hence, although these youngsters have not succeeded in achieving an upper secondary degree still by age 21, they have typically spent most of their early post-compulsory-school years as full-time students. Moreover, the share of non-completers following early study tracks has increased in all four countries over time, that is, when comparing the situation across the three youth cohorts covered by our national datasets. While less than $64 \%$ of the Nordic non-completers from the 1993 cohort followed either standard or delayed study tracks after leaving compulsory school, this share had increased to almost 70\% in the 2003 cohort. (Chapter 4)

When, as in Chapter 5, we refine our analysis of Nordic noncompleters by grouping them according to their main activity after three or less years in upper secondary education, full-time studies still retain their position as also non-completers' main type of activity: about $39 \%$ of Danish, close to $37 \%$ of Norwegian and Swedish and some $35 \%$ of Finnish non-completers go into early post-compulsory-school trajectories dominated by full-time studying during the five years up to age 20 . Another important type of early post-compulsory-school trajectory is characterised by the non-completer substituting school with work be- 
fore graduating from upper secondary school: almost 36\% of Danish non-completers show up in this type of employment-dominated early track with the corresponding share being close to $31 \%$ for Finland, almost $28 \%$ for Norway and about $25 \%$ for Sweden. However, while we observe a clear-cut increase across cohorts in the share of young noncompleters following standard study tracks, there seems to be no common Nordic trend for these school-dropout-employment tracks: larger shares of Swedish non-completers from the youngest (2003) cohort follow such employment tracks, whereas the situation is the opposite among Danish non-completers belonging to different cohorts. All in all, then, the results indicate, once again, that pathways dominated by school and work activities are the most prominent ones also among young non-completers and that this holds true for all four Nordic countries under study. (Chapter 5)

The rest of Nordic non-completers follow early post-compulsoryschool-dropout trajectories ending in so-called NEET activities, that is, unemployment or inactivity, including disability arrangements. Unemployment pathways are followed by almost $17 \%$ of Swedish noncompleters, about $12 \%$ of Finnish non-completers, over $9 \%$ of Norwegian non-completers, but less than $5 \%$ of Danish non-completers. Disability benefits are rare among young non-completers with the share being highest (some 4\%) for Sweden and lowest (only 1.3\%) for Denmark. In contrast, large shares of young people lacking an upper secondary degree still at age 21 have a history of post-compulsory-school trajectories dominated by other types of inactivity. This share is particularly high for Norway covering about one-fourth of the country's noncompleters with, moreover, a majority of them having dropped out only after three years in upper secondary school. The corresponding share is just below 20\% among Danish and Finnish non-completers, and about $17 \%$ among Swedish non-completers. (Chapter 5)

However, when the share of young non-completers having followed NEET-dominated tracks already at an early age is, instead, related to the full youth population, these quite large cross-country differences in NEET shares become much smaller: about $10 \%$ of both Danish and Norwegian youths are non-completers going early into NEET-dominated tracks, with the corresponding share being some 6\% for Finland and Sweden. Hence, the much lower completion rate among 21-year-olds observed for Denmark and Norway, when compared to Finland and Sweden, is not about young compulsory-school leavers going into NEETdominated tracks, but more about young people following study- or em- 
ployment-dominated tracks without achieving an upper secondary degree by age 21 . (Chapter 5)

Next, we explored labour market outcomes at age 21, 26 and 31, respectively. Of completers aged 21 , about $90 \%$ were found to be either studying or working. This high "activity" share among young completers is retained, and typically also strengthened, at age 26 , with more than $67 \%$ of Swedish completers being in employment compared to between 59 and $62 \%$ in the other three countries. By age 31, the share of completers in employment has increased to over $85 \%$ in Denmark and Sweden and to about $76 \%$ in Finland and Norway, whereas the share still enrolled in full-time education was mostly down at about $10 \%$. Conversely, the share of completers in NEET activities is in all four countries quite low, ranging from about $13 \%$ in Norway to less than $5 \%$ in Denmark. (Chapter 6)

The situation looks very different for non-completers, albeit a strikingly large proportion of also non-completers is studying or working in adulthood. At age 31, this share is close to $84 \%$ for Danish noncompleters, compared to about $74 \%$ for Swedish non-completers and some $70 \%$ for Finnish and Norwegian non-completers. However, among the non-completers we also find large shares of unemployed, disability beneficiaries and those having withdrawn into other types of inactivity. Norway stands out with an extraordinarily large share of noncompleters being outside both education and the labour market when aged 31, whereas Sweden is characterised by a large share of noncompleters in disability arrangements when young adults. Finland, in turn, has a comparatively large share of non-completers showing up as unemployed jobseekers as young adults, which mainly reflects the high unemployment level in the 1990s. (Chapter 6)

When these outcomes of young non-completers at three different age points - 21, 26, and 31, respectively - are, instead, related to the full youth population, the overall cross-country picture changes, once again, in certain crucial respects. Most notably, while we observe for both Denmark and Norway a comparatively high share of non-completers among the 21-year-olds, many of them are, nonetheless, in employment when young adults. At age 31 , about $27 \%$ of the Danish youth population investigated cover employed young people who were classified as noncompleters when aged 21. The corresponding share for Norway is around 19\%, but only some 10\% for Finland and Sweden. These highly different shares reflect well the cross-country variation in the number of young people completing an upper secondary degree only beyond age 21 and, in particular, the strikingly different labour market outcomes of 
these late completers in the four countries. Indeed, in Denmark it does not seem to matter that much if you complete your education by age 21 or 26, whereas it matters a lot if you are a non-completer still at age 31 . In Sweden, in contrast, it matters a lot if you fail to complete an upper secondary degree in the normal time, whereas Finland and Norway fall in-between these two extremes. Another distinct feature is the conspicuously similar share of the NEET population across the four countries despite remarkable cross-country differences not least in the share of young people still lacking an upper secondary degree when aged 21. (Chapter 6) Taken together, these findings suggest that cross-country differences in school-to-work-transition patterns are not necessarily a decisive determinant of the countries' NEET rates. Accordingly, the different upper secondary education systems in place should rather be judged by their implications for the development of skills and long-term productivity, as well as by their costs.

There is a huge body of literature providing support for the contention that school success and, ultimately, labour market outcomes are closely related to the young person's family background. Also our results support this broad-based evidence. It is, therefore, of interest to explore whether the link we observe between early and later school and labour market experiences is, ultimately, a link between family background and later labour market outcomes. Our results strongly suggest that this is not the case: the early post-compulsory-school trajectory followed by a young person plays a role also after account has been made for differences in family background. In particular, we observe growing so-called state dependence among non-completers having followed early tracks dominated by NEET activities: early unemployment shows up in an increasing risk of experiencing unemployment in adulthood; early disability arrangements tend to continue in adulthood; and early inactivity involves a high risk of continuing outside both education and the labour market also as a young adult, with disability arrangements often being the ultimate outcome. (Chapter 7) However, we do not claim that there is a causal relationship between early school and labour market experiences and later outcomes. The same underlying cause, such as a disability, may well affect both the pathway through upper secondary education and future labour market prospects. Still, the strong relationship identified between the early trajectory followed and subsequent outcomes most likely also includes causal elements implying that, regardless of the underlying cause, evidence-based information about young people's pathways that predicts undesirable outcomes may serve as an important source for better targeted policies towards youth. 
In sum, our report points to four major results:

A first major finding is large cross-country differences in terms of non-completion rates at different ages with non-completion from upper secondary education continuing well into the thirties. In Finland and Sweden, the non-completion rate is comparatively low already among 21-year-olds and converges towards $10 \%$ when young people are traced up to age 31. In Norway and especially in Denmark, the non-completion rate among 21-year-olds is much higher and has, by age 31 , converged towards $20 \%$. Indeed, completion from upper secondary school only beyond age 21 is especially prevalent among young Danes.

A second main finding emerging for all four countries is that, although non-completion predicts risky labour market outcomes in adulthood, employment and studying is by far the dominant activity over the next ten years or so also among those young people who fail to complete an upper secondary degree by age 21 . Hence, non-completion when aged 21 does not automatically imply that the young person is an early school-leaver or a school dropout having serious difficulties in coping with economic and social life.

A third major result is that the different pathways followed by young non-completers straight after completing compulsory school, are strongly related to their labour market outcomes in adulthood also after taking into account differences in family background. This implies that also evidence concerning young people's early school and labour market experiences contains valuable information for policies targeted at youth.

Last, but not least, our findings show that the distribution of youth into study and employment activities versus NEET activities converges in adulthood across the four Nordic countries. This suggests that the merits of different upper secondary education systems should perhaps, first and foremost, be judged by their implications for skills and income formation rather than by their merits in terms of "producing" NEETs. 


\section{References}

Abbott, A. (1983). Sequences of Social Events - Concepts and Methods for the Analysis of Order in Social Processes, Historical Methods 16, 129-147. http://dx.doi.org/10.1080/01615440.1983.10594107

Abbott, A. \& Forrest, J. (1986). Optimal Matching Methods for Historical Sequences, Journal of Interdisciplinary History 16, 471-494. http://dx.doi.org/10.2307/204500

Acemoglu, D. \& Autor, D.H. (2011). Skills, Tasks and Technologies: Implications for Employment and Earnings. In: O. Ashenfelter \& Card, D. (eds.), Handbook of Labor Economics, Volume 4B. Amsterdam: Elsevier. http://dx.doi.org/10.1016/s01697218(11)02410-5

Albæk, K. (2005). Om Lærepladsproblemet, Nationalokonomisk Tidsskrift 143, 1-25.

Albæk, K. (2009). The Danish Apprenticeship System, 1931-2002: The Role of Subsidies and Institutions, Applied Economics Quarterly 55(1), 39-60.

http://dx.doi.org/10.3790/aeq.55.1.39

Albæk, K. (2012). The Incidence of Employment Subsidies for Vocational Training, Applied Economics Quarterly 42, 93-109. http://dx.doi.org/10.3790/aeq.58.2.93

Albæk, K., Asplund, R., Barth, E. \& von Simson, K. (2014). Early school leaving and labour market prospects. Chapter 6 in T. Valkonen \& Vihriälä, V. (eds), The Nordic model - challenged but capable of reform. Copenhagen: TemaNord 2014:531, 235259. http://dx.doi.org/10.6027/TN2014-531

Asplund, R. (2009). The effect of labour market functioning on employment and unemployment. Helsinki: Ministry of Employment and the Economy, MEE Publications, Employment and Entrepreneurship 40/2009. (in Finnish with English summary).

Asplund, R. \& Vanhala, P. (2013). Nuorten työllisyydestä ja työttömyydestä hyvin vaihteleva kuva. Helsinki: ETLA Muistio 19 - 18.12.2013.

Asplund, R. \& Koistinen, P. (2014). Onko työmarkkinoilla tilaa kaikille? Katsaus erityisryhmiin kohdistetun politiikan tuloksiin ja haasteisiin. Helsinki: Työ- ja elinkeinoministeriön julkaisuja, Työ ja yrittäjyys, 22/2014.

Asplund, R. \& Vanhala, P. (2014). Peruskoulutodistuksen varassa olevien nuorten polut työelämään - 2000-luvun alussa peruskoulun päättäneiden kokemuksia. Helsinki: ETLA Muistio 23, 17.1.2014.

Barth, E. \& von Simson, K. (2012). Ungdomsarbeidsledighet og konjunkturer, Økonomiske analyser 5/2012.

Becker, G.S. (1964:) Human capital; a theoretical and empirical analysis, with special reference to education. National Bureau of Economic Research General series, Vol no 80. National Bureau of Economic Research; distributed by Columbia University Press, New York.

Bell, D.N.F. \& Blanchflower, D.G. (2009). What to do about rising unemployment in the UK? Bonn: IZA DP No. 4040. 
Björklund, A., Lindahl, L. \& Lindquist, M.J. (2010). What More Than Parental Income, Education and Occupation? An Exploration of What Swedish Siblings Get from Their Parents, B e Journal of Economic Analysis \& Policy, 10.

Black, S. \& Devereux, P. (2011). Recent developments in intergenerational mobility. In: O. Ashenfelter \& Card, D. (eds.), Handbook of Labor Economics. Amsterdam: Elsevier, 1487-1695. http://dx.doi.org/10.1016/s0169-7218(11)02414-2

Bragstad, T. \& Brage, S. (2011). Unge på arbeids- og helserelaterte ordninger. Oslo: NAV-rapport 1/2011.

Bratsberg, B., Raaum, O., Røed, K. \& Gjefsen, H.M. (2010). Utdannings- og arbeidskarrierer hos unge voksne: Hvor havner ungdom som slutter skolen i ung alder? Rapport 3/2010, Ragnar Frisch Centre for Economic Research.

Brunello. G. \& De Paola, M. (2014). The Costs of Early School Leaving in Europe, IZA Journal of Labor Policy 2014, 3:22, 1-11. http://dx.doi.org/10.1186/2193-9004-3-22

Brzinsky-Fay C. (2007). Lost in Transition? Labour Market Sequences of Schoolleavers in Europe, European Sociological Review 23(4), 409-422. http://dx.doi.org/10.1093/esr/jcm011

Brzinsky-Fay, C., Kohler, U. \& Luniak, M. (2006). Sequence analysis with Stata, Stata Journal 6, 435-460.

Bäckman, O., Jakobsen, V., Lorentzen, T., Österbacka, E. \& Dahl, E. (2011). Dropping out in Scandinavia. Social Exclusion and Labour Market Attachment among Upper Secondary School Dropouts in Denmark, Finland, Norway and Sweden. Arbetsrapport 2011:8, Institutet för Framtidsstudier.

Cedefop (2012). From education to working life: the labour market outcomes of vocational education and training.

http://www.cedefop.europa.eu/EN/publications/21556.aspx

Clark, D. (2011). Do Recessions Keep Students in School? The Impact of Youth Unemployment on Enrolment in Post-compulsory Education in England, Economica 78(311), 523-545. http://dx.doi.org/10.1111/j.1468-0335.2009.00824.x

COM (2010) 2020: Europe 2020. A strategy for smart, sustainable and inclusive growth. Brussels: Communication from the Commission of the European Communities, 3.3.2010.

COM (2010) 477: Youth on the Move - An initiative to unleash the potential of young people to achieve smart, sustainable and inclusive growth in the European Union. Brussels: European Commission, 15.9.2010.

COM (2010) 682: An Agenda for new skills and jobs: A European contribution towards full employment. Strasbourg: Communication from the Commission to the European Parliament, the Council, the European Economic and Social Committee and the Committee of the Regions, 23.11.2010.

COM (2011) 18: Tackling early school leaving. A key contribution to the Europe 2020 Agenda. Brussels: Communication from the Commission of the European Communities, 31.1.2011.

COM (2011) 19: Proposal for a Council Recommendation on policies to reduce early school leaving. Brussels, 31.1.2011. 
COM (2012) 495 final: Draft 2012 Joint Report of the Council and the Commission on the implementation of the renewed framework for European cooperation in the youth field (EU Youth Strategy 2010-2018). Brussels: Communication from the Commission to the European Parliament, the Council, the European Economic and Social Committee and the Committee of the Regions, 10.9.2012.

COM (2011) 933 final: Youth Opportunities Initiative. Brussels: Communication from the Commission to the European Parliament, the Council, the European Economic and Social Committee and the Committee of the Regions, 20.12.2011.

Engberg, J. (2014). Rekrytering, genomströmning och relevans - en studie av yrkesoch lärlingsutbildningssystemen $i$ Norden. Köpenhamn: Nordiska ministerrådet, TemaNord 2014:544. http://dx.doi.org/10.6027/TN2014-544

Eurofound (2012). NEETs - Young People not Employment, Education or Training: Characteristics, Costs and Policy Responses in Europe. Dublin.

Falch, T. \& Nyhus, O.H. (2009). Frafall i videregående opplæring og arbeidsmarkedstilknytning for unge voksne. Senter for økonomisk forskning AS.

Halvorsen, B., Hansen, O. J. \& Tägtström, J. (2012). Unge på kanten. Om inkludering av utsatte ungdommer. København: Nordisk ministerråd, TemaNord 2012:005. http://dx.doi.org/10.6027/Nord2012-005

Hardoy, I., Røed, K., Torp, H. \& Zhang, T. (2006). Virker ungdomsgarantien?, Søkelys på arbeidsmarkedet 23, 21-30.

Høst, H. (2008). Fag-og yrkesopplæringen i Norge - noen sentrale utviklingstrekk. Oslo: NIFU Step Rapport 20.

ILO (2011). Global Employment Trends for Youth: 2011 update. Geneva.

ILO (2013). Global Employment Trends 2013: Recovering from a second jobs dip. Geneva.

ILO-IMF (2010). The Challenges of Growth, Employment and Social Cohesion. Discussion document downloadable at http://www.osloconference2010.org/

Jakobsen, V. \& Liversage, A. (2010). Køn og etnicitet $i$ uddannelsessystemet litteraturstudier og registerdata. København: SFI - Det Nationale Forskningscenter for Velfærd, Rapport nr. 10:29.

Kahn, L.B. (2010). The long-term labor market consequences of graduating from college in a bad economy, Labour Economics 17(2), 303-316.

http://dx.doi.org/10.1016/j.labeco.2009.09.002

Larja, L. (2013). Nuorten elinoloja ei voi kuvata pelkän työttömyysasteen avulla, Hyvinvointikatsaus $1 / 2013,9-17$.

Lyche, C.S. (2010). Taking on the Completion Challenge: A Literature Review on Policies to Prevent Dropout and Early School Leaving. Paris: OECD Publishing, OECD Education Working Paper No. 53. http://dx.doi.org/10.1787/5km4m2t59cmr-en

Markussen, E. (Red.) (2010). Frafall i utdanning for 16-20 åringer i Norden. København: Nordisk ministerråd, TemaNord 2010:517. http://dx.doi.org/10.6027/TN2010-517

Martin, P. \& Wiggins, R.D. (2011). Optimal matching analysis. In: Willians, M. \& Vogt, W.P. (eds), SAGE Handbook of Innovations in Social Research Methods. Los Angeles: Sage, 385-408. http://dx.doi.org/10.4135/9781446268261.n22

Ministry of Education and Research (2000). Education Act 1985:1100. Stockholm.

Myrskylä, P. (2011a). Nuorten työttömyyden mittaaminen on vaikeaa, Tieto\&trendit 8/2011. 
Myrskylä, P. (2011b). Nuoret työmarkkinoiden ja opiskelun ulkopuolella. Helsinki: Työ- ja elinkeinoministeriön julkaisuja, Työ ja yrittäjyys 12/2011.

Möller, J. \& Umkehrer, M. (2014). Are There Long-Term Earnings Scars from Youth Unemployment in Germany?, ZEW Discussion Paper No. 14-089.

Müller, W. \& Gangl, M. (eds.) (2003). Transitions from education to work in Europe. The Integration of Youth into EU Labour Markets. Oxford: Oxford University Press. http://dx.doi.org/10.1093/0199252475.001.0001

Nilsen, Ø. A. \& Holm Reiso, K. (2014). Scarring effects of early-career unemployment, Nordic Economic Policy Review 1/2014, 13-46.

Nordström Skans, O. (2011). Scarring Effects of the First Labor Market Experience. Bonn: IZA Discussion Paper No. 5565.

OECD (2008a). Employment Outlook 2008. Paris.

OECD (2008b). Off to a Good Start? - A Descriptive Review of Youth Labour Market Transitions in OECD Countries. Paris.

OECD (2008c). Jobs for Youth: Norway. Paris: OECD Publishing.

OECD (2009). Employment Outlook. Paris: OECD Publishing.

OECD (2010a). Education at a Glance 2010. OECD INDICATORS. Paris: OECD Publishing.

OECD (2010b). Off to a Good Start? Jobs for Youth. Paris: OECD Publishing.

OECD (2010c). Jobs for Youth: Denmark. Paris: OECD Publishing.

Olofsson, J. \& Wadensjö, E. (2007). Ungdomar, utbildning och arbetsmarknaden $i$ Norden - lika men ändå så olika. Stockholm: Rapport från Forskningsrådet för arbetsliv och socialvetenskap.

Olofsson, J. \& Panican, A. (red.) (2008). Ungdomars väg från skola till arbetsliv - nordiska erfarenheter. Köpenhamn: Nordiska ministerrådet, TemaNord 2008:584. http://dx.doi.org/10.6027/TN2008-584

Prince Trust (2010). YouGov Youth Index 2010. http://www.princestrust.org.uk/pdf/Youth_Index_2010.pdf

Quintini, G. \& Manfredi, T. (2009). Going Separate Ways? School-to-Work Transitions in the United States and Europe. Paris: OECD Publishing OECD Social, Employment and Migration Working Paper, No. 90. http://dx.doi.org/10.1787/221717700447

Ramböll Management Consulting AB (2010). Nordiska länders insatser mot ungdomsarbetslöshet - kartläggning och analys. Köpenhamn: Nordiska ministerrådet, TemaNord 2010:570. http://dx.doi.org/10.6027/TN2010-570

Reiling, R.B. \& Strøm, B. (2015). Upper secondary school completion and the business cycle, The Scandinavian Journal of Economics 117(1), 195-219.

Räisänen, H. (2013). Suomessa talouskriisin vaikutuksia on vaimennettu ennen kaikkea lomautuksilla, Hyvinvointikatsaus 1/2013, 24-29.

Räisänen, H., Alatalo, J., Krüger Henriksen, K., Israelsson, T. \& Klinger, S. (2012). Labour market reforms and performance in Denmark, Germany, Sweden and Finland. Helsinki: Ministry of Employment and the Economy, Employment and Entrepreneurship 19/2012.

Saar, E., Unt, M. \& Kogan, I. (2008). Transition from Educational System to Labour Market in the European Union: A Comparison between New and Old Members, International Journal of Comparative Sociology 49(1), 31-60.

http://dx.doi.org/10.1177/0020715207088586 
Scarpetta, S., Sonnet, A. \& Manfredi, T. (2010). Rising Youth Unemployment During The Crisis: How to Prevent Negative Long-term Consequences on a Generation? Paris: OECD Social, Employment and Migration Working Papers No. 106.

Schmillen, A. \& Umkehrer, M. (2013). The Scars of Youth - Effects of Early-Career Unemployment on Future Unemployment Experiences. IAB Discussion Paper 06/2013.

SEC(2011) 96: Reducing early school leaving. Brussels: Commission Staff Working Paper, 31.1.2011.

SEC (2011) 401: On EU indicators in the field of youth. Brussels: Commission Staff Working Document, 25.03.2011.

Socialstyrelsen (2014). Ekonomiskt bistånd årsstatistik 2013. Stockholm. http://www.socialstyrelsen.se/publikationer2014/2014-6-22.

Wolbers M. (2007). Patterns of labour market entry: A comparative perspective on school-to-work transitions in 11 European countries, Acta Sociologica 50, 189-210. http://dx.doi.org/10.1177/0001699307080924

Von Simson, K. (2014). Explaining upper secondary school dropout: New evidence on the role of local labor markets, Empirical Economics. http://dx.doi.org/10.1007/ s00181-014-0829-3 



\section{Sammanfattning}

Unga i åldern 16-20 år gör ofta olika vägval när det gäller utbildning och planering inför sitt framtida yrkesliv. Samtidigt sägs detta vara den tidsperiod i livet som är den mest kritiska med tanke på senare arbetsmarknadsutfall. Med andra ord, de vägar som de unga händelsevis väljer under de första åren efter avslutad grundskola kommer högst sannolikt att påverka deras framtida möjligheter och utfall på ett avgörande sätt. Det centrala i föreliggande rapport har varit att undersöka hur dessa tidiga vägval ser ut, samt att utforska deras koppling till de ungas arbetsmarknadsutfall i vuxenlivet.

I vår studie följer vi tre kohorter av ungdomar: de som fyllde 16 år 1993, 1998 och 2003. Alla dessa ungdomar, som representerar totalt fyra nordiska länder - Danmark, Finland, Norge och Sverige, har följts fram till år 2008, vilket innebär att den längsta uppföljningsperioden omfattar 15 år.

I de nordiska länderna inleder en stor majoritet grundskoleelever fortsatta studier, antingen direkt eller efter ett mellanår, och studerar mer eller mindre oavbrutet fram till 20 års ålder, vanligtvis ännu längre. Många unga gör emellertid också en helt andra vägval, vilka ofta kantas av riskfyllda faser såsom längre perioder utanför både utbildning och arbetsmarknad. I våra analyser har vi använt en så kallad klusterteknik för att gruppera de unga i olika kluster beroende på vilka vägval de gjort efter avslutad grundskola.

Genom hela rapporten görs konsekvent en distinktion mellan unga som har fullföljt sina fortsatta studier efter avslutad grundskola senast vid fyllda 21 år, och unga som fortfarande endast har grundskoleexamen som 21-åringar.7 Tyngdpunkten i rapporten ligger på den senare kate-

\footnotetext{
${ }^{7}$ I vår rapport avser fortsatta studier efter avslutad grundskola det som på engelska kallas upper secondary education och som motsvarar ISCED 3. Generellt kan denna utbildningsnivå betecknas som utbildning på mellannivå efter avslutad utbildning på lägre och högre grundnivå (= grundskola). Denna utbildning på mellannivå är emellertid olika utformad också i de nordiska länderna och beskrivs dessutom med olika mer eller mindre officiella termer. I Danmark används numera termen "ungdomsuddannelse". I Finland talar man om "fortsatta studier efter grundskolan i den allmänbildande gymnasieutbildningen och yrkesutbildningen", medan man inom utbildningsförvaltningen talar om andra stadiet i motsats till grundnivå (=ISCED 1\&2). I
} 
gorin av unga. Dessutom lyfter vi fram skillnader mellan könen, liksom mellan de tre undersökta ungdomskohorterna.

När det gäller de nordiska länderna kan Danmark och Sverige närmast beskrivas som varandras motpoler i fråga om den fortsatta utbildningen efter avslutad grundskola, särskilt yrkesutbildningen. Danmark har ett välutvecklat system för lärlingsutbildning som ger de unga omfattande arbetsplatsförlagd praktik, medan yrkesutbildningen i Sverige i huvudsak kan beskrivas som skolbaserad, även efter reformen som trädde i kraft 2011. Denna skillnad i ländernas utbildningssystem efter avslutad grundskola avspeglas tydligt i de ungas levnadsbanor fram till 20 års ålder och därför även i andelen unga som vid en given ålder fullföljt sina studier efter avslutad grundskola. I Sverige fortsätter den typiska unga att studera i tre år efter avslutad grundskola och andelen unga som inte fullföljt sina fortsatta studier som 21-åringar är som högst $17 \%$ i de tre ungdomskohorterna. I Danmark, å andra sidan, är motsvarande andel unga så hög som 39 \% i 1998 års ungdomskohort (och endast något lägre i de andra två kohorterna). Situationen i Norge liknar mer den i Danmark än i Sverige, med en andel unga på som högst $32 \%$ som har enbart grundskoleexamen fortfarande vid 21 års ålder. Finland ser mer ut som Sverige än som Danmark eller Norge, med en andel på knappt 20 \% som inte ännu vid 21 års ålder avlagt någon examen efter avslutad grundskola.

Det är anmärkningsvärt att även när vi begränsar analysen till de unga som fortfarande som 21-åringar inte avlagt någon examen efter avslutad grundskola så visar det sig att en betydande del av dem fortsatt studera, men utan att lyckas slutföra sina studier inom fem år efter avslutad grundskola. När vi gör en gemensam nordisk klusteranalys för dessa ungdomar finner vi att $58 \%$ av de finländska ungdomarna har fortsatt sina studier efter avslutad grundskola, antingen direkt eller efter ett mellanår, men utan att ha avlagt examen som 21-åringar. Motsvarande andel för Danmark är ca $62 \%$, för Norge ungefär $71 \%$ och för Sverige nästan $76 \%$. Dessa andelar vittnar om att även om de här ungdomarna inte lyckats fullfölja sina fortsatta studier ännu vid 21 års ålder så har de oftast tillbringat största delen av tiden efter avslutad grundskola i utbildning. Den här andelen unga har dessutom ökat över tid i samtliga fyra länder, vilket framgår när man jämför situationen i de tre ungdomskohorter som vår studie bygger på. Knappt $64 \%$ av de nordiska unga i 1993 års kohort som fortfarande som 21-åringar inte avlagt 
någon examen efter avslutad grundskola hade fortsatt studera efter grundskolan antingen direkt eller efter ett mellanår. Andelen hade ökat till nästan 70 \% i 2003 års kohort.

En något mer detaljerad analys av dessa ungdomar utgående från deras huvudsakliga verksamhet efter tre eller färre år i fortsatta studier efter avslutad grundskola, utvisar att fortsatta studier är den övervägande vanligaste aktiviteten: i Danmark är ca $39 \%$, i Norge och Sverige nästan $37 \%$ och i Finland omkring $35 \%$ av dessa unga huvudsakligen i utbildning under de fem första åren efter avslutad grundskola. En annan viktig grupp bildar de unga som börjat arbeta istället för att fullfölja sina fortsatta studier efter avslutad grundskola: av de danska ungdomar som fortfarande som 21-åringar saknar examen från fortsatta studier dyker nästan $36 \%$ upp i den här jobbdominerade kategorin, medan motsvarande andel är knappt $31 \%$ för Finland, närmare $28 \%$ för Norge och ca 25 \% för Sverige. Men medan vi ser en tydlig ökning över tid av andelen unga som fortsätter i utbildning utan att avlägga examen, så förefaller det inte finnas någon gemensam nordisk trend för de unga som avbrutit sina fortsatta studier för arbete: i Sverige visar andelen unga med enbart grundskoleexamen som börjat jobba en ökning över tid, medan situationen är den motsatta bland de danska årskullarna. Överlag tyder resultaten på att ungas vägval efter avslutad grundskola domineras av antingen fortsatta studier eller jobb eller en kombination av dessa. De här vägvalen är de mest framträdande även bland de ungdomar som fortfarande som 21-åringar saknar examen efter avslutad grundskola. Dessutom upprepas samma mönster i samtliga fyra nordiska länder som ingår i vår studie.

Resten av de unga som fortfarande vid 21 års ålder saknar examen efter avslutad grundskola tenderar att höra till den så kallade NEETgruppen, vilket innebär att de antingen är arbetslösa eller befinner sig helt utanför arbetskraften (= inaktiva, inklusive unga med funktionshinder). Andelen 21-åringar med enbart grundskoleexamen som mestadels varit arbetslösa under de fem första åren efter avslutad grundskola är nära $17 \%$ i Sverige, medan motsvarande andel är ca $12 \%$ i Finland, drygt $9 \%$ i Norge men under $5 \%$ i Danmark. Förtidspensioneringar förekommer sällan; andelen är högst (ca $4 \%$ ) för Sverige och lägst (endast 1,3 \%) för Danmark. Däremot är andra former av inaktivitet mycket vanligt förekommande bland unga som fortfarande som 21-åringar saknar examen efter avslutad grundskola. Andelen är särskilt hög för Norge eller ca $25 \%$. För Danmark och Finland stannar andelen strax under $20 \%$, och för Sverige är den ca $17 \%$. 
Dessa relativt stora skillnader mellan de nordiska ländernas NEETandelar minskar dock märkbart om andelen unga som huvudsakligen följt NEET-dominerade vägar efter avslutad grundskola relateras till hela ungdomspopulationen och inte, som ovan, till populationen av unga som fortfarande som 21-åringar saknar examen efter avslutad grundskola: ca $10 \%$ av både danska och norska ungdomar kan karakteriseras som unga som har enbart grundskoleexamen på grund av att de i ett tidigt skede efter avslutad grundskola hamnat utanför både utbildning och arbetsliv. Motsvarande andel för Finland och Sverige är ca $6 \%$. Detta innebär att den betydligt lägre andelen danska och norska unga (jämfört med finländska och svenska unga) som fullföljt sina fortsatta studier senast vid 21 års ålder, inte bör tolkas som att danska och norska unga uppvisar en klart högre risk att hamna i en NEET-situation efter avslutad grundskola utan snarare som att de har en större tendens att fortsätta i utbildning eller börja jobba utan att ännu som 21-åringar ha avlagt examen efter avslutad grundskola.

Slutligen undersökte vi de ungas arbetsmarknadsutfall vid 21, 26 respektive 31 års ålder. Av 21-åringar med examen efter avslutad grundskola var ca $90 \%$ i antingen utbildning eller arbete. Samma eller ännu större andelar noterades för de högre åldrarna. Vid 26 års ålder var mer än $67 \%$ av de svenska ungdomar som avlagt examen efter avslutad grundskola senast som 21-åringar, sysselsatta jämfört med mellan 59 och $62 \%$ i de tre övriga länderna. Vid 31 års ålder hade andelen sysselsatta bland dessa unga ökat till över $85 \%$ i Danmark och Sverige och till ca $76 \%$ i Finland och Norge, medan andelen som fortfarande var i utbildning hade vanligtvis sjunkit till runt $10 \%$. Omvänt var NEETandelen bland dessa unga i alla fyra länder relativt låg, ca $13 \%$ i Norge och under $5 \%$ i Danmark.

Situationen ser helt annorlunda ut för de unga som ännu som 21åringar saknade examen efter avslutad grundskola, även om en påfallande stor del av också dessa ungdomar antingen studerar eller arbetar i vuxen ålder. Vid 31 års ålder är den här andelen närmare 84 \% för de danska ungdomar som fortfarande vid 21 års ålder hade enbart grundskoleexamen, medan motsvarande andel är ca $74 \%$ för Sverige och omkring 70 \% för Finland och Norge. Men i den här specifika ungdomsgruppen finner vi också många som vid 31 års ålder är arbetslösa, erhåller ersättning kopplat till funktionshinder eller befinner sig av andra orsaker utanför både utbildning och arbetsmarknad. Norge sticker ut med en mycket stor andel unga utanför utbildning och arbetsliv, medan Sverige kännetecknas av en stor andel som erhåller någon form av ersättning kopplat till funktionshinder. Finland åter har bland dessa unga 
en jämförelsevis stor andel som är arbetslösa som unga vuxna, något som i huvudsak speglar den höga arbetslösheten under 1990-talet och särskilt bland dem med enbart grundskoleexamen.

När vi istället relaterar dessa arbetsmarknadsutfall för unga som fortfarande som 21-åringar saknar examen efter avslutad grundskola till hela ungdomspopulationen, förändras dock återigen skillnaderna mellan länderna. Mest anmärkningsvärt är att trots den höga andelen danska och norska ungdomar som fortfarande vid 21 års ålder saknar examen efter avslutad grundskola så är många av dem de facto sysselsatta som unga vuxna. Vid 31 års ålder representerar ca $27 \%$ av den danska ungdomspopulationen som täcks av vår studie sysselsatta unga som vid 21 års ålder hade enbart grundskoleexamen. Motsvarande andel för Norge är omkring $19 \%$, men endast ca $10 \%$ för Finland och Sverige. Den här stora variationen i andelar avspeglar väl det faktum att länderna uppvisar väsentliga skillnader i antalet unga som fullföljer sina fortsatta studier först efter att de fyllt 21, och i all synnerhet att arbetsmarknadsutfallet för dessa unga är högst olika i de fyra länderna. I Danmark verkar det faktiskt inte spela så stor roll om man fullföljer sina fortsatta studier som 21- eller 26-åring, medan det däremot betyder en hel del om du fortfarande som 31-åring saknar examen efter avslutad grundskola. I Sverige, å andra sidan, är det av avgörande betydelse för arbetsmarknadsutfallet om du misslyckas med att fullfölja dina fortsatta studier inom normal tid. Finland och Norge placerar sig mellan dessa två extremfall. Ett annat anmärkningsvärt resultat är att NEET-andelen i ungdomspopulationen är i stort sett densamma i de fyra länderna trots de stora skillnaderna i andelen unga som fortfarande vid 21 års ålder saknar examen efter avslutad grundskola. Sammantaget tyder dessa resultat på att skillnader mellan länder i fråga om de ungas övergång från skola till arbetsliv inte nödvändigtvis är av avgörande betydelse för ländernas NEET-grad. Detta tyder i sin tur på att de olika systemen för fortsatta studier efter avslutad grundskola bör snarare utvärderas på basis av deras effekt på kunskapsutvecklingen och den långsiktiga produktiviteten, samt de kostnader de ger upphov till.

Det finns en omfattande litteratur som stöder antagandet att skolresultat och i sista hand arbetsmarknadsutfall är nära relaterade till den ungas familjebakgrund. Också våra resultat stöder detta antagande. Följaktligen är det av intresse att undersöka om sambandet vi ser mellan tidiga och senare utbildnings- och arbetsmarknadserfarenheter egentligen är en koppling mellan familjebakgrund och senare arbetsmarknadsutfall. Våra resultat tyder på att så absolut inte är fallet: de vägval som ungdomar gör efter avslutad grundskola är av stor betydelse också efter 
att vi kontrollerat för väsentliga skillnader i de ungas familjebakgrund. Framför allt ser vi tilltagande så kallat situationsberoende (eng. statedependence) bland unga som i ett tidigt skede efter avslutad grundskola hamnat i en situation som domineras av NEET-aktiviteter. Tidiga erfarenheter av arbetslöshet visar sig i form av en klart ökad risk att bli arbetslös som ung vuxen. Ersättningar kopplade till funktionshinder tenderar att fortsätta in i vuxenlivet. Tidiga erfarenheter av andra former av inaktivitet medför ökad risk att den unga stannar utanför både utbildning och arbetsmarknad också som ung vuxen, varvid ersättning till följd av funktionshinder ofta blir den slutliga lösningen. Därmed hävdar vi emellertid inte att det förekommer ett kausalt samband mellan tidiga utbildnings- och arbetsmarknadserfarenheter och senare utfall. Samma underliggande orsak, såsom exempelvis funktionshinder, kan mer än väl påverka både de ungas väg genom utbildningssystemet och deras framtida arbetsmarknadsutsikter. Likväl är det högst sannolikt att det starka samband som vi finner mellan de ungas tidiga vägval och senare utfall samtidigt avspeglar kausala element. Detta innebär i sin tur att, oberoende av den underliggande orsaken, så kan empiriskt framtagen information om ungas vägval som tenderar att leda till oönskade utfall användas som viktigt bakgrundsmaterial då man eftersträvar att föra en mer målinriktad ungdomspolitik.

Sålunda kan vår rapport sammanfattas i form av fyra huvudresultat:

Det första huvudresultatet pekar på stora skillnader mellan de fyra studerade nordiska länderna vad gäller unga som saknar examen efter avslutad grundskola; skillnaderna är stora vid olika åldrar och kvarstår också bland unga vuxna. I Finland och Sverige är andelen unga med enbart grundskoleexamen relativt liten redan bland 21-åringar och omkring $10 \%$ bland 31-åringar. I Norge, och i synnerhet i Danmark, är andelen 21-åringar utan examen efter avslutad grundskola betydligt större även om den sjunker till omkring $20 \%$ för 31-åringar. Det att man avslutar sina fortsatta studier först efter fyllda 21 är särskilt vanligt bland unga i Danmark.

En andra huvudsaklig slutsats som därtill gäller samtliga fyra länder är att även om avsaknaden av examen efter avslutad grundskola ökar risken för sämre arbetsmarknadsutfall i vuxen ålder, så är sysselsättning och utbildning de helt dominerande aktiviteterna ända upp i trettioårsåldern också bland de ungdomar som har enbart grundskoleexamen fortfarande vid 21 års ålder. Enbart grundskoleexamen ännu som 21åring innebär sålunda inte att den unga kan automatiskt klassas som en person som i ett tidigt skede lämnat all utbildning bakom sig eller som 
en person som avbrutit sina studier till följd av allvarliga ekonomiska och sociala problem.

Ett tredje viktigt resultat är att de tidiga vägval som de ungdomar gjort som fortfarande vid 21 års ålder saknar examen efter avslutad grundskola är starkt relaterade till deras arbetsmarknadsutfall som unga vuxna också efter att vi beaktat skillnader i ungdomarnas familjebakgrund. Detta innebär att empiriska resultat gällande ungdomars tidiga utbildnings- och arbetsmarknadserfarenheter kan bidra med värdefull information när det gäller att utforma åtgärder riktade mot unga.

Slutligen utvisar våra resultat att ungdomarna i de fyra studerade länderna fördelar sig rätt olika mellan dels studier och sysselsättning och dels NEET-aktiviteter, men också att dessa skillnader minskar i betydande utsträckning när ungdomarna når vuxen ålder. Detta antyder att fördelarna med olika utbildningssystem för fortsatta studier efter avslutad grundskola bör kanske i första hand utvärderas utgående från hur de påverkar kunskaps- och inkomstbildningen snarare än i vilken utsträckning de "producerar" NEET-unga. 



\section{Appendix: Descriptions of national datasets used}

\section{Denmark}

The Danish dataset used in the project is compiled from register data at Statistics Denmark. The dataset encompasses three cohorts of young Danish residents. The first cohort contains all residents, who were 16 years old in 1993, and they are followed until they are 31 years old, in 2008. The second cohort consists of Danish residents, who were 16 years old in 1998. They are followed up to age 26, in 2008. The third cohort consists of Danish residents, who were 16 years old in 2003, and they are followed until they are 21 years old, in 2008.

Statistics Denmark has supplied various kind of information from registers in Statistics Denmark for the young people in these three cohorts. The result is linked data constructed for use in the analyses presented in this report.

The main information used for the project is register-based labour force statistics (Registerbaseret Arbejdsstyrkestatistik - RAS) that assign each Danish resident to one and only one state in the Danish labour market at one particular point in time (the end of November each year). This statistic follows the international guidelines of the International Labour Organization (ILO) for assessments of the attachment of the population to the labour market. The ILO guidelines are primarily used in relation to interviews, where each person answers questions about the attachment to the labour market. RAS uses the guidelines to choose the appropriate labour market state. The Danish classification is rather detailed and presently contains 62 different categories, see http://www.dst.dk/da/Statistik/dokumentation/Times/ida-databasen/ ida-personer/pstill.aspx.

Another main source of information is statistics on education. These statistics contain the current and finished education on a detailed basis. We have also linked the young people in the three cohorts to information on parental background. This background includes the education and the income of the parents (as obtained from tax registers). 


\section{Finland}

The project uses the linked employer-employee data (Finnish Longitudinal Employer-Employee Data FLEED) that Statistics Finland has created for research use. The FLEED data consists of persons aged 15 to 70 living in Finland between 1988 and 2011 (excl. Åland). The persons have been followed over time, so there is data on the person for all the years during which the person has been alive, aged between 15 and 70 and residing in Finland. The FLEED data includes information on the person's basic characteristics, family, living, employment relationships, periods of unemployment, income and education.

The FLEED data is based on employment statistics that are annual statistics providing data by region on the population's economic activity and employment. The population for the statistics is the permanently resident population in the country on the last day of the year. The data are mainly derived from around 40 administrative registers and statistical data files.

The produced data describe the population's main type of activity, occupation, status in occupation, number of workplaces, location of workplace, and education and income of the population and labour force. The reference period of the statistics is the last week of the year, but the statistics also contain data accumulated during the statistical reference year (e.g. income data, months of employment and unemployment).

The population and definitions of the employment statistics have remained more or less the same since 1987, when regular production of the statistics was started. However, the classifications used in the statistics have changed along the years. For example, the Standard Industrial Classification was amended in 1993, 2001 and 2007 and the Classification of Occupations in 1995 and 2010. The changes in the classifications have an effect on the comparability of the years because it has not been possible to build complete conversion keys between all the classifications.

\section{Sources}

- http://tilastokeskus.fi/tup/mikroaineistot/ me_kuvaus_henkilo_en.pdf

- http://www.stat.fi/meta/til/tyokay_en.html 


\section{Norway}

The Norwegian analyses use data from FD-Trygd, an administrative database compiled by Statistics Norway. The database consists of several welfare and employment registers at the individual level, together with demographic information such as age, gender, parents' education and income, etc. Information about education is gathered from NUDB (Nasjonal Utdanningsdatabase). The different registers are linked together by a unique identification key, which makes it possible to follow individuals as they "move" between different labour market and welfare states, as well as to and from education.

The data used in this project encompasses all Norwegian residents who have started an upper secondary education during the period 1992-2008. This means that we lack information about youth who do not enrol in upper secondary education in this time period. The enrolment rate in Norway is very high - around $96-97 \%$ of each youth cohort enrol in upper secondary education the same year they finish compulsory school. Among those who do not have a direct transition from compulsory to upper secondary school, almost $70 \%$ have enrolled within five years after completing compulsory school (Falch and Nyhus, 2009). This means that the data covers practically the entire population of Norwegian youth, but the bias is likely to be larger for the younger cohorts as they are observed for fewer years. Table A1 shows the bias for the three youth cohorts used in this project.

\begin{tabular}{lrrr}
$\begin{array}{l}\text { Table A1. Youth cohorts in the data and full population, Norway } \\
\text { Number of youth in the } \\
\text { project data }\end{array}$ & $\begin{array}{r}\text { Number of youth in full } \\
\text { population }\end{array}$ & $\begin{array}{r}\text { Difference between data } \\
\text { and full population }\end{array}$ \\
$\begin{array}{l}\text { Cohort of } \\
\text { 16-year-olds }\end{array}$ & 51,012 & 51,282 & -270 \\
1993 & 51,394 & 52,029 & -635 \\
1998 & 53,758 & 55,524 & $-1,766$ \\
\hline
\end{tabular}

Notes: The full population numbers are gathered from Statistics Norway. All numbers comprise youth who turn 16 in 1993, 1998 or 2003 and who are resident in Norway the following five years.

The following registers are used in order to classify the youth into the five main activity categories: Ongoing education (Student); Employment (Employed); Maternity benefit and sickness benefit (Employed); Conscripts (Employed); Job seekers and occupationally handicapped (Unemployed); Rehabilitation and vocational rehabilitation allowance (Pensioner); Temporary disability benefits (Pensioner); Disability pension (Pensioner); Social assistance (Other). In order to make the data comparable to the other countries' data in this 
project, we use December as our reference month. The exception is ongoing education, which is measured in October each year.

\section{Sweden}

The Swedish data is based on several registers from Statistics Sweden. The major data source is LISA (Longitudinal integration database for health insurance and labour market studies), a longitudinal database covering education, income and employment. The population consists of all over 16-year-old nationally registered individuals during the years 1960-2012. This means that a large part of the Swedish population is followed over a long period of time. Information on the index persons' biological siblings, half siblings on each parent's side, spouses and all persons residing in the household is added to the database. The different registers are linked together by a unique identification key, which makes it possible to follow individuals as they move between different activities. The material also covers variables representing the individuals' demographic and socio-economic status. These additional variables make it possible to estimate the effects of failing or succeeding with various upper secondary educations, taking into account the individuals' sex, country of birth, parents' level of education, parents' level of income, etc. Thus, the effects of labour market change on young individuals' labour market careers can be pursued not only from a macro-economic perspective, but also from a detailed, geographical and longitudinal socio-economic perspective.

The Swedish data used in this project include the following main registers: Longitudinal integration database for health insurance and labour market studies; the multi-generational register; the income and wealth register, the education register and the total population register. 
Table A2. Summary of the number of young people in the four national datasets used

\begin{tabular}{|c|c|c|c|c|c|c|}
\hline \multirow[t]{2}{*}{ Total } & \multicolumn{3}{|c|}{ All three cohorts } & \multicolumn{3}{|c|}{ Cohort of 16-year-olds } \\
\hline & All & Males & Females & 1993 & 1998 & 2003 \\
\hline Denmark & 164,879 & 85,190 & 79,689 & 56,710 & 51,055 & 57,114 \\
\hline Finland & 193,567 & 99,211 & 94,356 & 65,595 & 67,068 & 60,904 \\
\hline Norway & 156,164 & 79,924 & 76,240 & 51,012 & 51,394 & 53,758 \\
\hline Sweden & 290,257 & 149,713 & 140,544 & 90,611 & 92,495 & 107,151 \\
\hline
\end{tabular}

\begin{tabular}{|c|c|c|c|c|c|c|}
\hline \multirow[t]{2}{*}{ Completers } & \multicolumn{3}{|c|}{ All three cohorts4 } & \multicolumn{3}{|c|}{ Cohort of 16 -year-olds } \\
\hline & All & Males & Females & 1993 & 1998 & 2003 \\
\hline Denmark & 103,203 & 49,046 & 54,157 & 36,932 & 31,071 & 35,200 \\
\hline Finland & 158,611 & 78,474 & 80,137 & 55,088 & 53,847 & 49,676 \\
\hline Norway & 109,723 & 53,000 & 56,723 & 36,451 & 36,434 & 36,838 \\
\hline Sweden & 243,763 & 123,438 & 120,325 & 77,498 & 76,790 & 89,475 \\
\hline
\end{tabular}

\begin{tabular}{|c|c|c|c|c|c|c|}
\hline \multirow[t]{2}{*}{ Non-completers } & \multicolumn{3}{|c|}{ All three cohorts } & \multicolumn{3}{|c|}{ Cohort of 16 -year-olds } \\
\hline & All & Males & Females & 1993 & 1998 & 2003 \\
\hline Denmark & 61,676 & 36,144 & 25,532 & 19,778 & 19,984 & 21,914 \\
\hline Finland & 34,956 & 20,737 & 14,219 & 10,507 & 13,221 & 11,228 \\
\hline Norway & 46,441 & 26,924 & 19,517 & 14,561 & 14,960 & 16,920 \\
\hline Sweden & 46,494 & 26,275 & 20,219 & 13,113 & 15,705 & 17,676 \\
\hline
\end{tabular}


Nordic Council of Ministers

Ved Stranden 18

DK-1061 Copenhagen K

www.norden.org

\section{Youth unemployment and inactivity}

Young people follow highly different trajectories from age 16 up to age 20, a time period which is often argued to be the most critical in terms of their future labour market outcomes. The focus of this report is on investigating the look of these early pathways, as well as on exploring their link to labour market outcomes in adulthood. Results are reported and compared for four Nordic countries: Denmark, Finland, Norway and Sweden. 